Travaux et documents du CIRAC

\title{
LES POLITIQUES
}

\section{D'INNOVATION COOPÉRATIVE}

\section{EN ALLEMAGNE ET EN FRANCE}

Expériences et approches comparées

Sous la direction de

Solène HAZOUARD, René LASSERRE, Henrik UTERWEDDE

\section{CIRAC}




\section{Les politiques d'innovation coopérative en Allemagne et en France}

Expériences et approches comparées

Solène Hazouard, René Lasserre et Henrik Uterwedde (dir.)

DOI : 10.4000/books.cirac.525

Éditeur : IFAEE

Année d'édition : 2010

Date de mise en ligne : 13 décembre 2017

Collection : Travaux et documents du CIRAC

ISBN électronique : 9782905518620

\section{Oonentition \\ Qbooks}

http://books.openedition.org

\section{Édition imprimée}

ISBN : 9782905518392

Nombre de pages : 260

\section{Référence électronique}

HAZOUARD, Solène (dir.) ; LASSERRE, René (dir.) ; et UTERWEDDE, Henrik (dir.). Les politiques d'innovation coopérative en Allemagne et en France : Expériences et approches comparées. Nouvelle édition [en ligne]. Cergy-Pontoise : IFAEE, 2010 (généré le 02 octobre 2020). Disponible sur Internet : <http://books.openedition.org/cirac/525>. ISBN : 9782905518620. DOI : https://doi.org/10.4000/ books.cirac.525

\section{(C) IFAEE, 2010}

Conditions d'utilisation:

http://www.openedition.org/6540 
Travaux et documents du CIRAC

\section{LES POLITIQUES}

\section{D'INNOVATION COOPÉRATIVE}

\section{EN ALLEMAGNE ET EN FRANCE}

\section{Expériences et approches comparées}

Sous la direction de

Solène HAZOUARD, René LASSERRE, Henrik UTERWEDDE 




\section{LES POLITIQUES \\ D'INNOVATION COOPÉRATIVE \\ EN ALLEMAGNE ET EN FRANCE}

Expériences et approches comparées

Sous la direction de

Solène HAZOUARD, René LASSERRE, Henrik UTERWEDDE

CIRAC

TRAVAUX ET DOCUMENTS DU CIRAC 


\section{Travaux et documents du CIRAC}

Collection dirigée par René Lasserre

Ouvrage publié avec le concours du Centre Interdisciplinaire d'Etudes et de Recherches sur l'Allemagne (CIERA) et de l'Université de Cergy-Pontoise (Civilisations et identités culturelles comparées des sociétés européennes et occidentales, CICC)
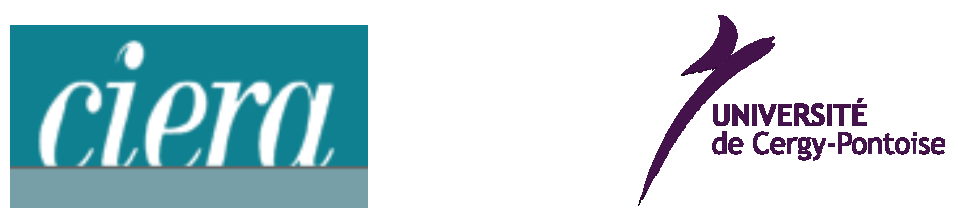

(C) CIRAC, 2010

www.cirac.u-cergy.fr

CIRAC, c/o Université de Cergy-Pontoise

33 Boulevard du Port - 95011 CERGY-PONTOISE CEDEX

ISBN 978-2-905518-39-2 


\title{
LES POLITIQUES D'INNOVATION COOPÉRATIVE en AlLEMagne et en France
}

\author{
EXPÉRIENCES ET APPROCHES COMPARÉES
}

\author{
TABLE DES MATIĖRES
}

REMERCIEMENTS

\section{INTRODUCTION}

Solène Hazouard, René Lasserre, Henrik Uterwedde

Echange d'expériences et de bonnes pratiques.

Contribution à une concertation franco-allemande en matière d'innovation .

I - STRUCTURATION INSTITUTIONNELLE DES SYSTÈMES

DE RECHERCHE ET D'INNOVATION .

Ulrich Schmoch

Structure du système de recherche et d'innovation allemand :

spécificités et appréciation.

Peter Weingart

Eléments du système allemand d'innovation : forces et faiblesses

Jean-Yves Mérindol

Le système d'innovation français face au modèle allemand

II - LES POLITIQUES DE SOUTIEN À L'INNOVATION.

\section{Rainer Nägele}

La stratégie « Hautes Technologies » de l'Allemagne : objectifs et réalisations.

\section{Sonja R. Kind}

La stratégie « Hautes Technologies » de l'Allemagne

et la promotion des clusters et réseaux.

\section{Mark Sellenthin}

Coordination et harmonisation de la politique d'innovation aux différents échelons politiques. L'exemple de la Saxe...

Bernard Froment

Enjeux et perspectives de la politique des pôles de compétitivité en France 


\section{Pierre Papon}

Stratégies des acteurs publics français et dynamiques locales :

les dilemmes du jacobinisme

III - CLUSTERS ET RÉSEAUX TERRITORIAUX

Thierry Weil

Les transferts de technologies en France

Petra Moog, Sebastian Hanny

Réseaux d'innovation en Allemagne - Principes généraux et exemples pratiques

de Rhénanie du Nord-Westphalie.

Horst Domdey

Le pôle biotech de Munich : une « culture de l'innovation»

Hubert Steigerwald

Le facteur humain au cœur du réseau bavarois Sensorik

IV - LES PME AU CCEUR DES PROCESSUS D'INNOVATION 169

Marianne Kulicke

Fonction des PME dans le système d'innovation allemand:

la politique de soutien au Mittelstand

Emmanuel Leprince

Le rôle des PME dans les pôles de compétitivité français

Michael Groß

Le capital-risque pour les petites et moyennes entreprises (PME) innovantes

Christoph Thieme

Petites et moyennes entreprises, moteurs de croissance et d'innovation

Conditions et perspectives du rôle des PME dans le cluster optique de Thuringe

Isabelle Bourgeois

L'intégration de la problématique du Wissensmanagement

V - MISE EN PERSPECTIVE EUROPÉENNE

Dieter Rehfeld

Politique de clusters : des expériences de gouvernance politique.

Alain Quévreux

L'intégration européenne des stratégies nationales 


\section{REMERCIEMENTS}

Cet ouvrage collectif vient clore un projet de formation recherche intitulé Le système allemand d'innovation : organisation et dynamiques nouvelles. Eléments pour une comparaison France-Allemagne. Mis en œuvre par le Centre d'Information et de Recherche sur l'Allemagne contemporaine (CIRAC) en coopération avec le Deutsch-Französisches Institut de Ludwigsburg (DFI) sur la période 2006-2008, ce projet avait pour ambition de mettre en évidence les facteurs de performance du système national allemand d'innovation, dans une démarche comparative franco-allemande. Il s'adressait aux responsables industriels nationaux et régionaux, à la communauté scientifique française, aux experts du sujet ainsi qu'aux doctorants et étudiants de niveau Master.

Les articles regroupés dans cette publication sont essentiellement issus des contributions que des acteurs et spécialistes de l'innovation, français et allemands, ont présentées au cours de deux journées d'étude et d'un colloque final. A l'occasion de cette dernière rencontre de dimension européenne, le groupe de travail FutuRIS de l'Association Nationale de la Recherche et de la Technologie (ANRT) a coopéré activement à la réflexion et aux travaux, contribuant largement, du côté français, à la comparaison des approches et à la qualité des débats.

Dans la réalisation de ce projet, les organisateurs ont bénéficié, pour une grande partie, du concours financier apporté par le Centre Interdisciplinaire d'Etudes et de Recherches sur l'Allemagne (CIERA) dans le cadre de ses Programmes de formation-recherche, auquel s'est associé le Centre de recherche "Civilisations et identités culturelles comparées des sociétés européennes et occidentales" (CICC) de l'Université de CergyPontoise. Outre les auteurs et les traducteurs, nous tenons à remercier Marie Pedron, assistante de direction et Cécile Santoul, documentaliste au CIRAC, pour l'aide précieuse qu'elles ont apportée à la mise en forme de cet ouvrage.

René Lasserre

Directeur du CIRAC
Henrik Uterwedde

Directeur adjoint du DFI
Solène Hazouard

Ingénieur d'études au CIRAC 



\title{
Echange d'expériences et de bonnes pratiques. Contribution à une concertation franco-allemande en matière d'innovation
}

\author{
Solène HAZOUARD, René LASSERRE, Henrik UTERWEDDE
}

L'innovation se situe au cœur des stratégies économiques contemporaines. Dans un contexte de changements profonds et accélérés par la mondialisation et l'émergence de nouveaux concurrents, par les mutations technologiques et les perspectives ouvertes par l'économie de la connaissance, enfin par le changement climatique, les problèmes écologiques et les défis du développement durable, les économies européennes ont un besoin impérieux de développer leur capacité d'innovation pour faire face à ces défis multiples. II y va de leur compétitivité, du maintien de leur potentiel d'emploi et de croissance et, audelà, de leur capacité de préserver, tout en les adaptant, leurs modèles économiques et sociaux. Or, l'échec de la stratégie de Lisbonne proclamée par l'Union européenne en 2000 montre bien que, si l'enjeu est véritablement européen, l'innovation reste largement le fait de variables, de structures et de politiques nationales. Alors qu'ils doivent répondre à des défis de dimension transnationale voire globale, les systèmes et les politiques d'innovation restent ancrés dans les trajectoires et dynamiques nationales ou infranationales, notamment dans leurs déclinaisons territoriales. Toute stratégie européenne d'innovation doit donc se nourrir des expériences nationales et régionales, extrêmement diverses et riches d'enseignements. A l'échelle des Etats membres, l'examen approfondi des bonnes pratiques des pays partenaires contribuera à l'élaboration d'une stratégie d'innovation communautaire mieux partagée et plus efficiente, génératrice de compétitivité.

L'Allemagne, qui dispose du plus important potentiel industriel d'Europe, et dont l'économie est de longue date, dès avant la Première Guerre mondiale, très largement ouverte sur le marché international, s'est toujours distinguée, depuis le renouveau économique des années 1950 , par sa capacité permanente à innover, que ce soit en termes de développement de nouveaux produits ou d'adaptation de ses structures de production et de commercialisation. Mais au-delà de cette dynamique « incrémentale », l'Allemagne s'attache depuis bientôt deux décennies à promouvoir une modernisation systémique de son dispositif de recherche et d'innovation, non seulement en y consacrant des moyens accrus, articulés autour d'axes stratégiques significatifs, mais également en mettant en œuvre de nouvelles régulations entre pouvoirs publics, recherche, milieux professionnels et entreprises.

Le dernier classement rendu public par la Commission européenne (European Innovation Scoreboard 2009), évaluant la capacité d'innovation de 33 pays, attribue du reste à l'Allemagne une encourageante quatrième place, la situant dans le petit groupe des « leaders d'innovation », ce qui tend à prouver que la politique menée en RFA porte ses fruits. 
Le résultat de cette étude, qui place la France en $11^{\mathrm{e}}$ position parmi les pays " suiveurs" en matière d'innovation, alors qu'elle est la seconde économie européenne et l'une des plus étroitement imbriquées à celle de l'Allemagne, justifie la pertinence d'une réflexion sur les performances et les spécificités du système d'innovation allemand. Toutefois, au-delà de leurs performances respectives, la France et l'Allemagne s'inscrivent dans un espace économique plus large, celui de l'UE qui, par sa taille et sa capacité d'intégration est seule en mesure de rivaliser avec les grandes puissances mondiales, au premier rang desquelles, aujourd'hui encore, les Etats-Unis et, demain, les puissances émergentes. Face à ce défi de la mise en commun des potentiels d'innovation nationaux à l'échelle européenne, le benchmark France-Allemagne constitue un point de passage obligé pour comprendre et concilier les dynamiques d'innovation respectives, renforcer les coopérations et impulser les synergies francoallemandes qui contribueront à la compétitivité globale de l'UE.

$\mathrm{Au}$ regard de cet enjeu décisif, le présent ouvrage se propose de rendre compte des orientations et des démarches mises en œuvre dans le cadre de la politique allemande d'innovation et de les mettre en regard avec celles développées en France. L'approche que nous avons choisi de retenir n'est cependant pas celle d'une étude d'ensemble du système allemand d'innovation et encore moins d'une étude systémique comparée Allemagne-France qui, l'une et l'autre, auraient relevé d'une ambition démesurée et nous auraient peut-être fait passer à côté de l'essentiel.

Dans sa singulière complexité, liée à son architecture institutionnelle multiniveaux, sa gouvernance multi-acteurs et à l'extraordinaire enchevêtrement de compétences qui en découle, le système d'innovation allemand résiste à toute logique cartésienne. Son intérêt et son efficacité résident ailleurs, moins dans la cohérence institutionnelle d'un dispositif, que dans la «manière de faire ». En fait ils se situent dans l'opérativité fonctionnelle d'un cadre politique d'ensemble indicatif et concerté qui à la fois définit des orientations lisibles et stables tout en mobilisant des moyens conséquents, mais qui, pour l'essentiel, laisse au libre jeu d'acteurs multiples le soin de définir et de mettre en œuvre sur le terrain les stratégies qu'ils jugent les plus appropriées. Et rien ne permet mieux d'illustrer cette logique coopérative de pilotage et d'action pragmatiques que l'analyse, à partir d'exemples concrets, de pratiques ou de stratégies reconnues de portée significative.

Quant à la comparaison franco-allemande, elle laisse le plus souvent l'observateur et l'évaluateur des politiques hexagonales désarmés quand, à l'aulne des pratiques de terrain, elle met en évidence l'irréductible distance que l'on relève entre d'un côté la rationalité construite des objectifs proclamés et des procédures institutionnelles mises en place et, de l'autre, leur efficacité toute relative en 
termes de résultats obtenus. L'approche de terrain dans l'analyse des pratiques françaises ne se révèle pas moins heuristiquement féconde et instructive. Notamment en ce qu'elle permet, par contraste, de mettre en évidence combien, dans la conduite des politiques publiques, la place réservée à l'autonomie et à l'exercice de la responsabilité des acteurs pourrait être un levier de réussite essentiel si l'on ne s'ingéniait sans cesse, par réflexe tutélaire, à en limiter la portée.

Fidèle à cette conviction fondée par l'expérience et selon laquelle l'initiative laissée aux acteurs est le véritable ressort de l'efficacité des systèmes, l'approche qui a guidé la conception et la réalisation de ce volume l'est également dans le choix délibéré qui a été le nôtre de ne donner aux analyses d'ensemble qu'une place mesurée. Nous avons ainsi privilégié largement les études de terrain, territoriales ou sectorielles, pour rendre compte des pratiques et des enjeux actuels des politiques d'innovation de part et d'autre du Rhin. N'y avait-il pas meilleur moyen, dans cette démarche qui vise à mettre en évidence les conditions propices à l'innovation, que de donner la parole aux acteurs de l'innovation eux-mêmes ?

Le présent recueil est issu des travaux de deux journées d'étude et d'un colloque organisés en 2007-2008, dans le cadre d'un projet de formation-recherche soutenu par le Centre Interdisciplinaire d'Etudes et de Recherches sur l'Allemagne (CIERA) sur le thème Le système allemand d'innovation : organisation et dynamiques nouvelles. Eléments pour une comparaison France-Allemagne. Ainsi la période d'analyse s'achève-t-elle aux prémices de la crise économique. Si certains développements ont eu cours depuis, les différents exposés demeurent néanmoins plus que jamais d'actualité, mettant en lumière le fonctionnement des politiques d'innovation mises en œuvre de part et d'autre du Rhin, les modes de financement des start-up ou encore le maillage des acteurs territoriaux au sein de réseaux d'innovation (ou clusters).

L'ensemble des contributions des auteurs allemands - les analyses des chercheurs comme les témoignages des acteurs impliqués directement dans les réseaux d'innovation - permet de mettre en évidence les facteurs de performance du système national allemand d'innovation et d'identifier les démarches concrètes engagées pour corriger la dégradation, enregistrée dès le milieu des années 1990, de la position internationale de l'Allemagne en matière d'innovation et pour satisfaire, au tournant du millénaire, aux objectifs de la stratégie de Lisbonne tracés par l'Union européenne. Cette analyse à multiples voix du système allemand est complétée, en contrepoint, par un certain nombre de contributions ou témoignages portant sur la politique d'innovation française, qui permettent un double regard contrastif franco-allemand. Cette approche se révèle effectivement, à la lecture, plus vivante et plus parlante, mais aussi, il faut bien en convenir, plus impressionniste et moins exhaustive. 
La première partie de l'ouvrage porte sur la structuration institutionnelle des systèmes de recherche et d'innovation. Il en ressort qu'un système d'innovation est porté par une dynamique complexe, à la fois économique et sociale, mais aussi politique, culturelle et historique, et qu'il faut prendre en compte ces facteurs systémiques afin de pouvoir cerner spécificités, forces et faiblesses d'un système d'innovation et s'inspirer de l'expérience du voisin. Il faut donc aller audelà, en amont des benchmarks quantitatifs habituels des performances et des politiques d'innovation, ainsi que des mesures concrètes, pour les placer dans leur contexte afin de bien comprendre les fonctionnements, les dynamiques et les contraintes spécifiques. C'est particulièrement vrai pour l'Allemagne, Etat fédéral au sein duquel la recherche et l'innovation relèvent de la double compétence du Bund et des Länder, ce qui implique que la répartition des compétences et des moyens procède d'un jeu particulièrement complexe. Mais cela ne vaut pas moins pour un Etat centralisé comme la France où la multiplication des instances et des opérateurs, l'empilement des procédures, sont loin de satisfaire aux principes d'efficacité et de transparence.

Les politiques concrètes de soutien à l'innovation sont abordées dans la deuxième partie. Celle-ci revient sur les mesures adoptées dans le cadre de la stratégie «Hautes Technologies » de l'Allemagne. Lancée en 2006 par le gouvernement fédéral, elle répond à trois grandes priorités, rappelées au fil des contributions :

- promouvoir l'émergence de marchés pilotes pour des produits et des services innovants ;

- rapprocher la science et l'industrie afin de commercialiser plus rapidement des produits et des services ;

- rendre les conditions cadre plus favorables pour donner davantage de sécurité et de liberté d'action aux chercheurs et aux innovateurs dans la réalisation de nouvelles idées.

Cette stratégie s'applique à 17 technologies clés (telles les nanotechnologies, les biotechnologies et les TIC) et 5 domaines transversaux (notamment le renforcement des liens science-industrie, le soutien au transfert de technologies et à la création d'entreprises), l'objectif principal étant de mobiliser les moyens nécessaires pour faciliter le passage de l'idée à l'innovation. Outre ces grandes orientations prises à l'échelle fédérale et en vertu du principe de subsidiarité, la politique d'innovation allemande se pratique à quatre niveaux décisionnels (à l'échelle des communes, des Länder, du Bund et de l'Union européenne), requérant ainsi l'existence de mécanismes de coordination. Cette décentralisation des efforts d'innovation laisse une place considérable à l'initiative des acteurs (issus de l'enseignement supérieur, des organismes de recherche extrauniversitaires et de l'industrie). Les stratégies sont développées et mises en œuvre de préférence à partir du terrain, dans une logique bottom-up. En France 
en revanche, non seulement les orientations, mais aussi les procédures de mise en œuvre en matière d'innovation sont souvent définies « d'en haut», en raison d'une culture et d'une pratique centralisatrices plus prononcées, et conditionnent fortement le jeu des acteurs impliqués. La politique des pôles de compétitivité, mise en place en 2004 en France, se donne certes pour objectif principal et délibéré de promouvoir la coopération entre acteurs sur le terrain en vue d'encourager les transferts et mises en commun de technologies. Elle procède en cela d'une approche nouvelle, dont la dynamique se révèle prometteuse, mais sans toutefois se départir d'un «directivisme incitatif» que les praticiens ne manquent pas de relever.

La troisième partie souligne l'importance des clusters et réseaux territoriaux dans les processus d'innovation. La concentration géographique des acteurs y est propice aux transferts de savoirs et savoir-faire, corollaire d'une coopération réussie entre les entreprises d'un côté et, de l'autre, l'enseignement supérieur et les centres de recherche extra-universitaires. Le propos est ici enrichi d'une étude de cas régionale (menée en Rhénanie-du-Nord Westphalie) et du témoignage des responsables de deux clusters bavarois (BioM et Sensorik, opérant respectivement dans le domaine des biotechnologies et des capteurs). Là encore, l'avis d'un expert français en matière de pôles de compétitivité et de transferts de technologies offre des pistes de réflexion pour une comparaison franco-allemande, en soulignant à la fois les différences d'approche et l'intérêt qu'il y aura à les faire converger par des coopérations bilatérales concrètes. Chantier à suivre, à partir d'initiatives régionales ou sectorielles qui s'amorcent progressivement.

La quatrième partie est consacrée à la place des PME, dont le rôle est primordial dans le développement et la commercialisation de produits innovants. Dans un premier temps, ce chapitre place le curseur sur les politiques de soutien au Mittelstand, ces entreprises de taille moyenne ou intermédiaire qui constituent le chaînon essentiel de l'économie allemande. Au-delà des aides publiques, les start-up et PME innovantes ont recours à un financement privé (sous forme de capital-risque) afin de poursuivre leurs activités de R\&D et d'être compétitives à l'échelle internationale. Car, à l'avènement de la société de la connaissance, seule une entreprise disposant des fonds nécessaires se trouve en mesure de faire circuler en interne savoirs et savoir-faire, vecteurs d'innovation et de croissance. Dans cet ensemble thématique, les défis inhérents à la reconversion industrielle est-allemande sont également évoqués (voir la contribution portant sur le rôle des PME dans le cluster optique de Thuringe). La problématique française est quant à elle abordée sous l'angle du Pacte PME, programme géré depuis 2006 par l'Agence française de l'innovation OSEO et le Comité Richelieu, qui représente l'association française des PME innovantes. 
Dans une cinquième et dernière partie, les auteurs s'interrogent sur l'intégration européenne des stratégies nationales d'innovation, explicitant par la même occasion le mode de gouvernance et la politique des clusters. Si les clusters doivent être susceptibles de créer de l'innovation en leur sein, ils doivent aussi être ouverts sur l'extérieur pour faciliter la circulation des savoirs et des compétences. Ces deux préalables constituent la condition sine qua non à leur bon développement. La dernière contribution de l'ouvrage porte sur l'émergence d'une économie de la connaissance à l'échelle mondiale, et sur la coordination des politiques d'innovation entre les Etats membres de l'UE.

EN PRÉSENTANT LA STRUCTURATION ET LE FONCTIONNEMENT du système d'innovation allemand - mis en regard avec son équivalent français -, nous avons souhaité informer le lecteur avisé et/ou curieux sur les bonnes pratiques qui résultent de ce modèle, tout en mettant en lumière les différences binationales. Car si les mesures adoptées dernièrement dans les deux pays ciblent des objectifs similaires, leur mise en œuvre diverge en raison de contextes structurels et temporels différents. Ainsi, tandis que la grande majorité des clusters français a été initiée tout récemment «d'en haut» sous l'incitation et l'encadrement fermes des pouvoirs publics, l'Allemagne privilégie une démarche d'appel à projets beaucoup plus ouverte, fondée sur une approche « d'en bas » qui s'appuie sur l'initiative et l'expérience partagées des réseaux d'acteurs préexistants.

Au-delà de la mise en évidence et de la compréhension des différences, l'enjeu consiste désormais à déceler et promouvoir la concertation et l'interopérabilité entre les approches française et allemande, en tirant profit de l'expérience des pratiques, et notamment en montrant l'importance que comporte l'implication effective des acteurs. Ce défi prend tout son sens dans la perspective de la politique européenne, et notamment de la stratégie Europe 2020 qui, plus modeste que la stratégie de Lisbonne, se concentre sur cinq thématiques: l'emploi, la recherche et l'innovation, le changement climatique et l'énergie, l'éducation et la lutte contre la pauvreté.

La concertation franco-allemande ne doit pas être uniquement le fait des décideurs politiques nationaux, mais impliquer également davantage les acteurs scientifiques, économiques et territoriaux, à l'instar de ce qui se pratique outreRhin et reste le point fort du système allemand de recherche et d'innovation. A travers ce livre, nous avons voulu montrer concrètement, en nous appuyant sur la parole des acteurs, l'intérêt du cas allemand et tracer les voies d'une meilleure convergence entre deux pays partenaires de choix qui devraient s'attacher, mieux qu'ils ne l'ont fait jusqu'à présent, à mettre en commun leurs potentiels considérables de recherche et d'innovation, au profit de la compétitivité et de l'avenir de l'Europe. 
$-1-$

STRUCTURATION INSTITUTIONNELLE DES SYSTĖMES

DE RECHERCHE ET D'INNOVATION 



\title{
Structure du système de recherche et d'innovation allemand : spécificités et appréciation
}

\author{
Ulrich SCHMOCH
}

Conformément aux objectifs de la stratégie de Lisbonne, chaque Etat membre de l'Union européenne doit consacrer à l'horizon 2010 3\% de son PIB aux activités de R\&D. Or qu'en est-il outre-Rhin ? Via une approche comparative internationale, Ulrich Schmoch, chef de projets à l'Institut Fraunhofer de recherche sur les systèmes et l'innovation (ISI) de Karlsruhe, révèle dans un premier temps l'origine institutionnelle et sectorielle des dépenses de R\&D. Sa contribution permet par la suite d'identifier les différents acteurs du système de recherche et d'innovation allemand ainsi que les défis qui se posent à eux, avec une attention particulière portée sur les centres de recherche extra-universitaires (Instituts Fraunhofer, Instituts Max-Planck...), les établissements d'enseignement supérieur et les instituts dits associés (An-Institute).

Le concept de «système national d'innovation » (SNI) a été maintes fois remis en question : la mondialisation s'étant considérablement intensifiée, il semble problématique de souligner les aspects nationaux. Mais si tous les pays sont confrontés à des défis mondiaux, chacun les aborde de manière spécifique. Dans ce contexte, s'intéresser à la dimension nationale consiste à s'interroger sur la part des étrangers travaillant dans un pays et à se demander si les entreprises d'un pays donné investissent ou exportent beaucoup à l'étranger.

Je travaille depuis de nombreuses années dans un service consultatif du gouvernement fédéral appelé "Capacité technologique de l'Allemagne». Lorsque nous avons démarré nos activités au début des années 1980, nous nous préoccupions exclusivement des entreprises et de leurs performances, c'est-àdire des exportations de biens de haute technologie, de la production ou de la recherche au sein des entreprises. Au fil du temps, il est devenu de plus en plus évident que cela ne suffisait pas et qu'il fallait également s'intéresser à l'ensemble du système de recherche et d'innovation, à la politique et aux infrastructures d'innovation. Les activités de la recherche financée par des fonds publics sont, elles aussi, très importantes. D'abord orienté sur les entreprises, notre service a développé un intérêt pour les systèmes nationaux d'innovation.

Notre propos est de mettre en évidence les principales structures de recherche et d'innovation allemandes comparées à celles de la France, mais aussi d'autres pays. Pour ce qui est des grandes caractéristiques, les chiffres de l'OCDE pour l'année 2004 montrent par exemple la part du financement public dans l'ensemble de la recherche (OCDE, 2006). En Allemagne, ce taux est de $30 \%$, un faible pourcentage comparé à celui observé en France $(37,5 \%)$, un pays présentant une structure semblable à celle des Pays-Bas, où la part de financement public est aussi relativement élevée. 
Le secteur de la défense est bien moins important en Allemagne qu'en France; dans des pays comme les Pays-Bas, la recherche dans ce domaine est insignifiante.

\section{Répartition institutionnelle et sectorielle de l'effort de recherche}

Pour ce qui est de la structure institutionnelle, on observe en Allemagne une part très élevée de la recherche en entreprise par rapport à la France et aux PaysBas (voir graphique 1). En Allemagne, la recherche publique est répartie de manière à peu près équilibrée entre institutions universitaires et organismes extra-universitaires. La part des organismes extra-universitaires était par le passé un peu supérieure à celle des universités; elle leur est aujourd'hui légèrement inférieure. Le volume du secteur extra-universitaire représente actuellement environ $80 \%$ de celui de la recherche universitaire.

Graphique 1 : Part relative des secteurs institutionnels dans les dépenses de R\&D pour un échantillon de pays, 2004

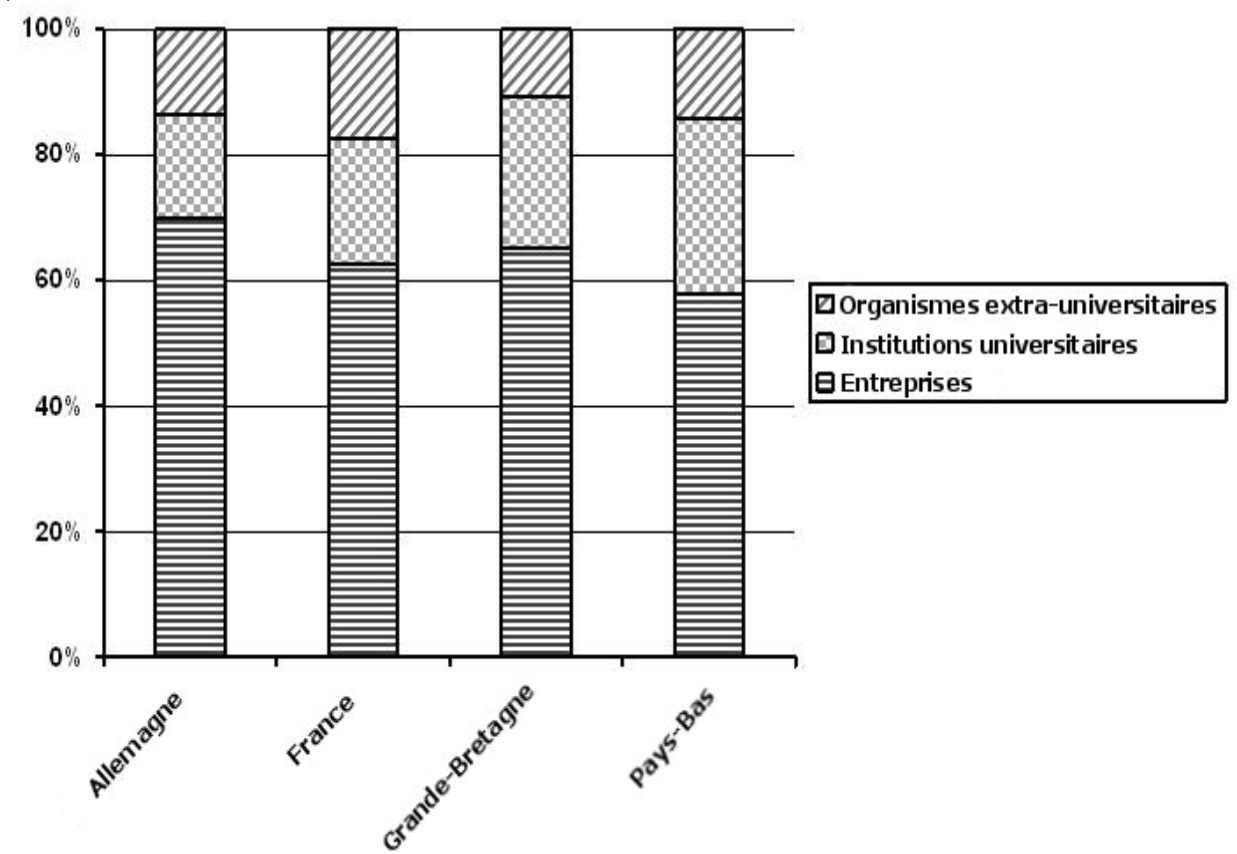

Source : OCDE (2006).

Ces structures sont assez semblables à celles qui existent en France. Mais la part du secteur de la défense est relativement élevée dans les organismes de recherche extra-universitaires français, ce qui est également le cas en GrandeBretagne. Dans ces deux pays, la part de la recherche civile dans les organismes 
extra-universitaires est en revanche relativement faible. La comparaison internationale montre par ailleurs que la part de la recherche universitaire dans l'ensemble du système est nettement plus élevée aux Pays-Bas qu'en Allemagne ou en France.

En Allemagne, les dépenses de recherche ont connu une très forte augmentation depuis les années 1960. On a observé un repli de la recherche en entreprise au début des années 1990 ; aujourd'hui, la courbe repart légèrement à la hausse. Parallèlement, la recherche dans les établissements d'enseignement supérieur et dans les organismes extra-universitaires n'a cessé d'augmenter, dans une dynamique constante. Cette importance croissante de la recherche - en particulier dans le secteur industriel - s'inscrit dans une tendance internationale qui vaut au moins pour les pays industriels. Cette croissance est toutefois plus faible en Allemagne que dans de nombreux autres pays. La Suède, la Finlande, les Etats-Unis et, ces dernières années à nouveau, le Japon sont nettement plus dynamiques.

Cette observation a bien entendu un lien avec les structures industrielles. S'agissant de l'objectif des $3 \%$ du PIB consacrés aux dépenses de R\&D défini à Lisbonne par l'Union européenne, les investissements de recherche correspondent surtout aux efforts réalisés dans le secteur industriel et moins à ceux effectués dans le secteur public, bien qu'un rôle important incombe à ce dernier. Depuis de nombreuses années, l'industrie allemande est dotée de structures de spécialisation très stables, longtemps dominées par la mécanique et la chimie traditionnelle. Ces quinze dernières années, le secteur automobile a connu une mutation importante. L'industrie chimique était jadis la plus puissante en matière de recherche. Elle a été détrônée par le secteur automobile : sans celui-ci, la part de la recherche dans le produit national brut (PNB) allemand serait très inférieure à celle qu'elle représente dans les autres pays industrialisés. Les chiffres plus élevés par exemple en Finlande, en Suède ou aux Etats-Unis tiennent aux structures industrielles dans ces pays. Le poids des technologies de l'information, des biotechnologies et du secteur pharmaceutique y est plus important - des secteurs de recherche qui n'existent pas sous cette forme en Allemagne. Seule une restructuration radicale au sein de l'industrie pourrait permettre d'atteindre l'objectif des $3 \%$, ce qui n'est pas réaliste. Il est également clair que les technologies de l'information requièrent un plus grand investissement en $R \& D$ que, par exemple, la mécanique ou la chimie traditionnelle. Mais leur part absolue est si faible qu'il faudrait des taux de croissance extraordinairement élevés pour atteindre l'objectif des $3 \%$. Il faut donc être prudent dans les proclamations politiques de cet objectif. Avec notre structure industrielle, ce n'est pas réaliste : il faudrait une mutation radicale. Et, pour faire passer les dépenses de recherche - qui représentent aujourd'hui environ $2,5 \%$ du PNB - à $3 \%$, il faudrait aussi du personnel qualifié, dans un secteur qui connaît des goulets d'étranglement. 


\section{Les grands organismes de recherche extra-universitaires}

\section{Graphique 2 : Les principaux organismes de recherche allemands et leurs dépenses de R\&D en 2004}

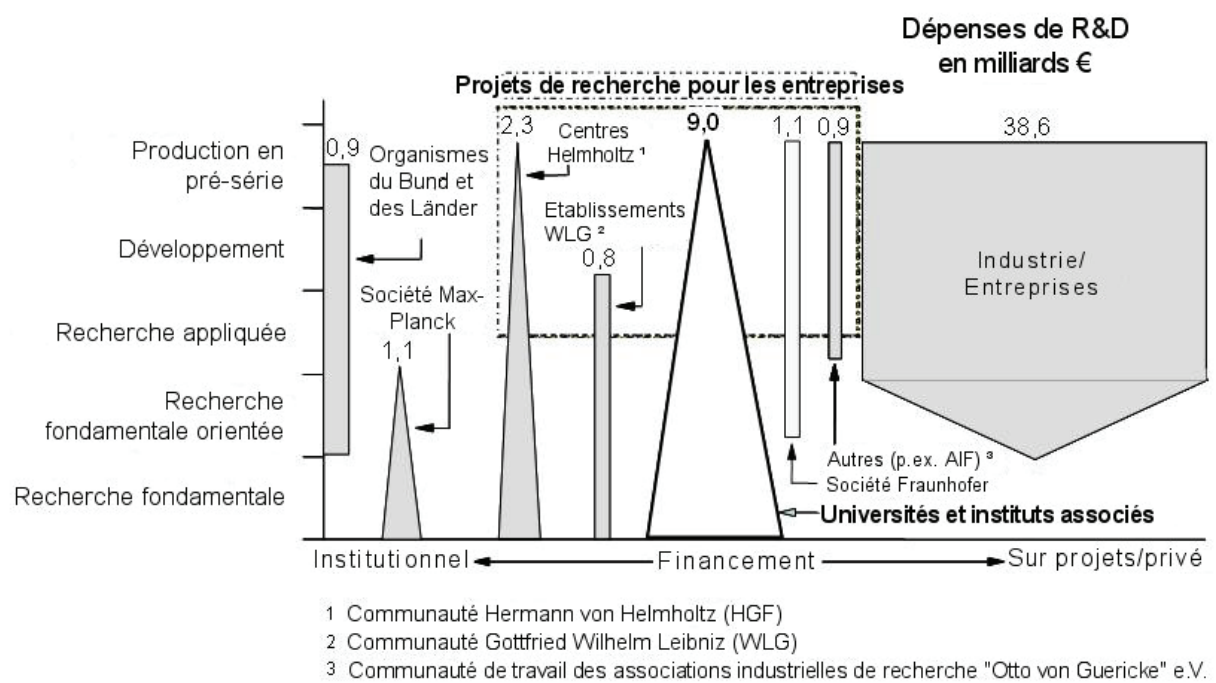

Source : BMBF (2006).

Le graphique 2 donne une représentation synthétique des structures du système de recherche allemand. Les organismes de recherche y sont représentés sur un plan à deux axes. Sur l'axe horizontal apparaît à gauche, à un extrême, le financement complètement institutionnel; à droite, le financement exclusivement sur des fonds privés et des fonds sur projets (Drittmittel). L'axe vertical va de la recherche purement fondamentale aux prototypes techniques, représentant ainsi dans son déroulement une recherche de plus en plus appliquée. Les différents organismes de recherche figurent sur ce diagramme, l'industrie représentant le bloc quantitativement le plus important. Ce sont bien entendu les dépenses de développement qui y sont le plus élevées. Mais si nous considérons uniquement la recherche fondamentale dans les entreprises, elle représente environ le même volume que la recherche universitaire (Schmoch, 2003, p. 198).

A gauche, en bas du diagramme, on trouve la Société Max-Planck, largement financée par des fonds institutionnels - et ne réalisant donc que peu de recherche avec des moyens alloués sur contrat - et essentiellement axée sur la recherche fondamentale. On trouve de l'autre côté, à l'extrême inverse, la Société Fraunhofer, fortement orientée application et financée pour une large part sur des fonds privés (environ $40 \%$ des moyens). Apparaissent au centre les universités, avec une prédominance de la recherche fondamentale, mais également une part non négligeable de recherche appliquée et de développement. 
Ce diagramme fait apparaître la situation du paysage scientifique pour l'année 2004. Si j'avais montré la situation d'il y a dix ans, les universités se seraient trouvées bien plus près de la Société Max-Planck. Aujourd'hui, la part des financements sur projets est considérable dans la recherche universitaire, ce qui signifie que les universités se rapprochent de plus en plus des entreprises.

L'Allemagne est un pays fédéral. L'éducation et donc les universités sont du ressort des Länder, et la récente réforme du système fédéral a clairement réaffirmé cette situation. Le Bund a tenté après la Seconde Guerre mondiale d'être plus présent dans la recherche, surtout via les organismes de recherche extrauniversitaires. On voit par exemple dans le cas de la Société Max-Planck que la répartition du financement entre Bund et Länder est de 50-50. Les instituts Max-Planck sont assez clairement tournés vers la recherche fondamentale, vers des thèmes de recherche d'excellence. Il existe 80 instituts dédiés aux sciences de la nature, de la vie, de l'ingénieur et aux sciences humaines. Il faut remarquer que la Société Max-Planck reçoit des fonds très largement institutionnels surtout par rapport à la Société Fraunhofer. Elle n'a pratiquement pas à rechercher de financements sur projets, ce qu'elle justifie en faisant valoir qu'elle pratique une recherche fondamentale d'excellence. Toutefois dans certains domaines thématiques, le secteur de la recherche sur les matériaux ou dans celui du génie génétique par exemple, les chercheurs de la Société Max-Planck estiment eux-mêmes qu'il faudrait aujourd'hui davantage de recherche appliquée. Cela leur paraît important car la recherche en biotechnologie ne peut selon eux être menée indépendamment de l'application. Mais ces réflexions ne sont pas formulées aussi clairement par la direction de la Société Max-Planck car une telle évolution estomperait les spécificités de cet organisme par rapport aux autres institutions de recherche.

Avec 59 instituts et 9000 collaborateurs, la Société Fraunhofer est un peu plus petite que la Société Max-Planck. Elle est dédiée à la recherche appliquée et au transfert de savoir vers les entreprises. Son financement est complètement différent de celui de la Société Max-Planck. Les ressources publiques de base ne représentent en effet que $34 \%$ de ses moyens, avec des variations possibles d'un institut à un autre. La Société Fraunhofer est financée par les entreprises à hauteur de $40 \%$, par le biais de projets de recherche, et $26 \%$ des fonds proviennent de projets publics remportés en concurrence avec des tiers. La dotation de base se répartit entre Etat fédéral et Länder avec un ratio de 90-10. Il faut souligner que ces ressources ne sont pas attribuées automatiquement, mais en fonction des moyens issus des entreprises. Cela signifie qu'un institut Fraunhofer ne trouvant pas suffisamment de fonds provenant de l'industrie n'obtient pratiquement pas de dotation. Il y a donc une forte incitation à trouver des ressources provenant de l'industrie. On constate par conséquent qu'à la Société Fraunhofer, le nombre de publications par chercheur est comparativement faible, alors que le nombre de dépôts de brevets y est élevé. On observe exactement l'inverse à la Société Max-Planck. 
$\mathrm{Au}$ sein de la Communauté Helmholtz, la part de la dotation de fonctionnement est également élevée. Cet organisme, doté d'un budget de 2,4 milliards $€$ pour l'année 2004 et comptant 22200 collaborateurs, est principalement né de la recherche sur l'énergie nucléaire. L'idée était de consacrer d'importants moyens à ce secteur, comme cela avait été fait avec le Projet Manhattan. Structurellement, la Communauté Helmholtz est comparable au Commissariat à l'énergie atomique (CEA). Puis, au milieu des années 1980, il fut décidé que la recherche nucléaire n'allait plus être une priorité. Cela n'a toutefois pas entraîné la suppression des grands centres de recherche, qui ont été dédiés à d'autres domaines. Mais le personnel a été réduit, de même que les activités. Dans le paysage de la recherche extra-universitaire, la Communauté Helmholtz reste l'organisme le plus important. Sa mission est moins claire que celle des sociétés Fraunhofer ou Max-Planck. On ne trouve essentiellement que deux arguments convaincants : d'une part, la Communauté Helmholtz se consacre à des projets de grande envergure qui ne pourraient être réalisés dans des universités. C'est également au sein de cette structure que l'on trouve les équipements lourds de la physique comme les accélérateurs de particules. D'autre part, les centres mènent une recherche dite "préventive», d'utilité publique, comme dans les secteurs de la santé et de l'environnement. Il y a depuis quelques années des débats de fond pour savoir si un tel système a encore un sens dans la mesure où une part considérable des fonds publics alloués à la recherche est attribuée à la Société Helmholtz alors que, dans le même temps, d'autres institutions déplorent un manque de moyens.

La Communauté Leibniz (WGL) regroupe 84 instituts, dont la mission est globalement moins claire. Cette structure est issue de plusieurs instituts auxquels a été reconnu un intérêt public particulier; ils sont financés pour moitié par le Bund et pour l'autre moitié par les Länder. Par le passé, ces organismes étaient dits instituts de la «liste bleue ». Ils se sont regroupés en une communauté afin de peser davantage dans le champ de la politique scientifique. Ces instituts sont pour partie des organismes de recherche et, pour partie, des institutions de services ; il peut par exemple s'agir de musées de sciences naturelles ou de centres d'information spécialisée (Fachinformationszentren). Compte tenu du débat actuel, il est tout à fait possible que, dans dix ans par exemple, de plus en plus d'instituts Leibniz soient associés à la Société Max-Planck, à la Société Fraunhofer ou à la Communauté Helmholtz, et que la Communauté Leibniz disparaisse. Cette question reste toutefois controversée, d'autant que de nombreux instituts Leibniz ont une excellente réputation scientifique.

\section{Une recherche universitaire davantage financée sur projets...}

Pour ce qui est des établissements d'enseignement supérieur, l'Allemagne ne dispose pas, à la différence de la France, de " grandes écoles »; elle compte en revanche 100 universités, dont 17 fortement axées sur les sciences de l'ingé- 
nieur. Les anciennes écoles polytechniques (technische Hochschulen) ont intégré de nombreuses autres unités de recherche, devenant ainsi des universités techniques. Il existe en outre un grand nombre de Fachhochschulen (168), des établissements plus petits que des universités, qui travaillent surtout dans les domaines de la gestion et des sciences de l'ingénieur et sont largement dédiés aux sciences appliquées. Ces établissements présentent une certaine ressemblance avec les «instituts universitaires de technologie (IUT)» en France.

La part des fonds sur projets dans la recherche universitaire n'a cessé d'augmenter depuis 1980. On observe un glissement structurel continu, la dotation de fonctionnement diminuant tandis que les ressources sur contrat augmentent. Un aspect important dans ce contexte est celui de l'augmentation des moyens attribués via la Communauté allemande pour la recherche (DFG). L'objectif est d'introduire davantage de compétition dans l'octroi de moyens publics. Au plan stratégique, il faut de nouveau penser au ratio entre ressources émanant de l'Etat fédéral et des Länder. La dotation des universités pour l'enseignement et la recherche provient des Länder, tandis que le financement de la DFG émane du Bund. L'augmentation du financement issu de la DFG implique par conséquent une plus grande influence de l'Etat fédéral. L'accroissement des moyens alloués sur contrat peut en tout cas être considéré comme le plus grand changement structurel affectant la recherche dans les universités.

\section{Graphique 3 : Origine des fonds sur projets (Drittmittel) des universités, 1997}

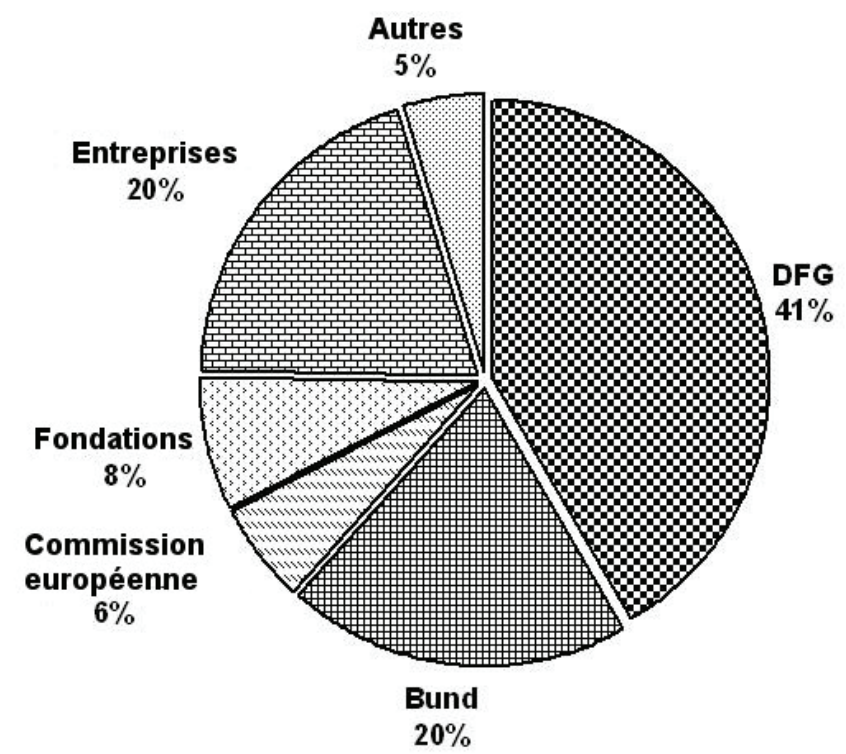

Source : Wissenschaftsrat (2000). 
Sur l'origine de ces fonds sur projets, on ne dispose malheureusement pas de données plus récentes que celles de l'année 1997, alors que les principaux changements sont intervenus depuis. En 1997, la DFG représentait une part considérable de $40 \%$, avec une priorité donnée à la recherche fondamentale. Depuis 1997, la part de l'Etat fédéral - alors de $20 \%$ - a augmenté. Il s'agit essentiellement de recherche correspondant à des axes définis par le ministère de la recherche, actuellement ministère fédéral de l'Enseignement et de la Recherche (BMBF). Il est certain que la part des projets financés par la Commission européenne - qui était de $6 \%$ en 1997 - a connu une nette augmentation. La part des fondations - au premier rang desquelles se trouve la fondation Volkswagen - n'a en revanche probablement pas augmenté depuis 1997 ; la part des fonds sur projets provenant d'entreprises, alors de $20 \%$, a connu une nette augmentation.

Selon les statistiques de l'OCDE sur la part des financements industriels dans l'ensemble de la recherche universitaire - dotations incluses -, avec $13 \%$, l'Allemagne dépasse tous les autres pays européens, mais également le Japon et les Etats-Unis. Seule la Corée du Sud présente un pourcentage légèrement supérieur. L'actuelle stratégie " Hautes Technologies » du BMBF a instauré un nouveau type de financement, la « prime de recherche », permettant à des organismes publics menant des recherches pour des petites et moyennes entreprises (PME) d'obtenir des moyens supplémentaires. Au début des années 1990, avant la création de cette prime, la part des moyens issus de l'industrie dans les ressources des universités était de $8 \%$ environ; elle est aujourd'hui de $13 \%$. L'incitation à rechercher davantage de financements privés consistait surtout par le passé à réduire les dotations ; ce n'était pas un élément de promotion de la politique d'innovation. La prime de recherche, destinée en particulier aux PME, revient de facto à financer de petits projets. Cela signifie que les chaires d'universités doivent gérer, outre l'enseignement et la recherche fondamentale, de nombreux petits projets, ce qui pourrait avoir des répercussions négatives sur la recherche fondamentale.

Les Fachhochschulen, fortement axées sur la pratique, sont bien souvent les partenaires idéaux pour les PME. Pour ces établissements, la prime de recherche pourrait aussi être un dispositif incitatif intéressant dans la mesure où ils ne disposent pas de dotation pour la recherche et ont un urgent besoin de tout financement supplémentaire.

\section{...et externalisée via les An-Institute}

La multiplication des instituts dits associés (An-Institute) au sein des universités constitue une évolution intéressante de ce point de vue. Une étude vient de recenser 544 instituts de ce type, dont $56 \%$ ont une orientation technique (Koschatzky et al., 2007). Ces instituts ont ceci de particulier qu'ils disposent d'une autonomie juridique - ils ne font donc pas partie des universités -, mais 
leur sont associés par le biais de contrats de coopération. En général, le directeur d'un An-Institut est aussi professeur dans l'université voisine. Cette organisation permet un transfert d'information et de personnel très poussé. Ces instituts ont été créés pour donner davantage de flexibilité dans le recrutement $\mathrm{du}$ personnel ou dans la réalisation de projets et de commandes. Leur financement et leur forme juridique sont très variables, mais leur structure fondamentale rappelle celle de la Société Fraunhofer: le financement se compose d'une dotation émanant des Länder, d'une série de projets de recherche pour des commanditaires publics et d'une part relativement élevée de fonds émanant de l'industrie (30\%) - au moins pour les An-Institute techniques. Avec 5000 salariés au total, cette structure est tout à fait importante, comparée par exemple aux instituts Fraunhofer, qui regroupent 8500 personnes. Les An-Institute perçoivent environ $43 \%$ des fonds alloués à l'université par l'industrie. Ces dernières années, les universités ont été soumises à une forte pression les incitant à coopérer avec des entreprises, le cadre juridique des universités étant toutefois souvent critiqué pour son manque de souplesse. Il y a régulièrement dans les universités un conflit entre la recherche fortement appliquée, axée sur le court terme et tournée vers l'industrie, et la recherche fondamentale, axée sur le long terme. C'est dans ce contexte que le modèle des An-Institute s'est développé, avec une croissance forte dans les années 1990.

Les An-Institute constituent un tissu parallèle à la Société Fraunhofer qui s'est largement développé de lui-même jusqu'en 2000. Ces dernières années, cette croissance a ralenti, mais on observe de nouveau une légère progression aujourd'hui. On envisage dans certains cas d'évaluer les An-Institute et de demander si les conditions qui avaient entraîné leur création il y a environ dix ou quinze ans sont toujours les mêmes ou bien s'il faudrait aller jusqu'à les fermer. Le transfert de technologie est d'ailleurs de plus en plus souvent un critère d'évaluation des universités, cette activité étant inscrite depuis quelques années dans les lois régionales sur l'enseignement supérieur comme troisième mission, à côté de la recherche et de l'enseignement. Les universités sont donc soucieuses de ramener dans leur giron des activités actuellement externalisées dans les An-Institute afin de pouvoir afficher davantage de transfert de technologie. On peut s'interroger sur la pertinence de cette approche. Car, pour permettre un transfert de technologie à grande échelle, les administrations des institutions doivent être organisées de façon nettement plus souple qu'elles ne le sont aujourd'hui. Les directeurs des An-Institute estiment en tout cas qu'il leur est plus facile de se rapprocher des entreprises dans le cadre de ces instituts que dans celui de l'université. A vrai dire, ce sont les An-Institute, mais aussi d'autres structures comme les entreprises reconnues d'utilité publique, des fondations ou d'autres formes d'organisations à la marge des universités, qui jouent un rôle majeur en matière de transfert de technologie. D'un point de vue statistique, cette réalité constitue une zone grise impossible à chiffrer précisé- 
ment. Toujours est-il que le transfert de technologie pratiqué par les universités est en réalité bien plus important que les financements industriels ne le laissent penser.

\section{Universités et organismes de recherche extra-universitaires : les enjeux actuels}

Le soutien à la recherche est pratiquement le terrain d'un rapport de forces entre Etat fédéral et Länder. Le Bund tente, en finançant des organismes extra-universitaires, d'accroître son poids dans la politique de recherche, et s'efforce par ailleurs d'exercer davantage d'influence sur les universités. La dernière action en ce sens est l'initiative dite «d'excellence » (Exzellenzinitiative), qui met des moyens considérables à la disposition des universités les plus performantes. Entre 2006 et 2010, 380 millions $€$ annuels doivent être dépensés, soit tout de même $30 \%$ des ressources de la recherche universitaire actuelle. Ce dispositif a donné lieu à une querelle de plusieurs années sur les compétences de l'Etat fédéral et des Länder, les deux parties ayant fini par se mettre d'accord sur un modèle commun de financement.

Du point de vue organisationnel, les universités sont actuellement touchées par une autre restructuration entraînant un renforcement de leur autonomie aux plans financier et administratif. Ce changement n'a pas encore été mis en œuvre partout en Allemagne, et de nombreuses universités déplorent une forte limitation de facto de leur autonomie par les contrats-cadre que les Länder tentent d'imposer.

Une seconde tendance, loin d'être appliquée partout et mise en œuvre différemment d'un Land à l'autre, consiste à renforcer les fonctions centrales, c'est-à-dire le recteur ou la présidence. Ce changement donne lieu actuellement à un processus très laborieux. Dans l'université classique, les décisions émanent largement de commissions universitaires au sein desquelles les enseignants négocient, au niveau des facultés, les stratégies à suivre. Ce système a fait l'objet de nombreuses critiques, car il fonctionne comme une sorte de pacte de non-agression, un professeur ne pouvant sauver son propre budget que s'il n'attaque pas trop durement ses collègues. Le nouveau système, plus centralisé, doit permettre de changer la donne. Il doit notamment créer la possibilité - et cela a également été formulé par la Conférence des recteurs allemands (HRK) de donner aux différentes universités des profils plus spécialisés. Cela signifie de facto fermer les filières faibles et renforcer les filières dominantes. Par conséquent, les universités ne proposeront plus, comme par le passé, toutes les disciplines; chacune d'elle fera un choix. La direction devra pour ce faire disposer des compétences de décision correspondantes. Reste à savoir d'où proviennent les informations qui serviront de base à ces décisions. C'est un processus difficile au quotidien, un processus qui requiert une gouvernance 
complètement nouvelle. Globalement, le système universitaire s'achemine en tout cas vers plus de concurrence.

La structure fédérale de l'Allemagne va de pair avec une structuration à petite échelle, chaque Land ayant ses propres lois sur l'enseignement supérieur. Il est par exemple très difficile pour un étudiant de quitter un Land pour un autre car les règlements des études diffèrent. Dans le cadre du renforcement des compétences des Länder sur les universités, on débat aussi de ce qui va advenir de petites disciplines comme la sinologie. Se pose la question, du point de vue des universités et des Länder, de savoir si de telles disciplines doivent être maintenues et, en cas de réponse négative, de savoir quelle université allemande continuera de les proposer. Se pose aussi la question du maintien des filières dont les diplômés ne trouvent pas de travail dans leur propre Land. Citons l'exemple de l'Université technique d'Illmenau, en Saxe-Anhalt, qui prépare de très bons diplômés, lesquels s'installent massivement dans le BadeWurtemberg. Une sorte de péréquation financière entre les Länder est en cours de discussion. Tous ces débats ont commencé à la suite de la récente réforme du fédéralisme et dureront certainement encore quelques années.

Les changements se poursuivent également dans les organismes extrauniversitaires. De nouvelles initiatives surgissent régulièrement dans les institutions dont la mission est moins évidente. Citons par exemple le «soutien de programme» de la Communauté Helmholtz, visant à lutter contre la tendance à la stagnation, souvent liée à une forte dotation de fonctionnement, en instaurant davantage de concurrence entre les différents centres et leurs instituts.

Enfin, on envisage actuellement un regroupement des universités et des organismes de recherche extra-universitaires. La comparaison avec les EtatsUnis fait ressortir que les universités américaines sont puissantes car elles ont intégré des structures qui, en Allemagne, fonctionnent comme des organismes extra-universitaires. Dans le cadre de l'Initiative d'Excellence, l'Université de Karlsruhe a été retenue avant tout pour avoir proposé une coopération institutionnelle plus étroite avec le centre de recherche de Karlsruhe - qui fait partie de la Communauté Helmholtz -, avec l'objectif ambitieux de fonder dans les années qui viennent le KIT (Karlsruhe Institute of Technologie). Depuis quelques années, l'Université et le centre de recherche de Karlsruhe coopèrent de manière approfondie et efficace dans le domaine des nanotechnologies. Les entretiens avec les acteurs directs montrent que les deux structures ont un grand intérêt à ce rapprochement. D'un côté, l'université assure la formation des futurs chercheurs; de l'autre, le centre Helmholtz dispose de moyens de recherche considérables, que l'université ne pourrait avoir à elle seule.

Les dispositions actuelles permettent à un professeur de l'Université de Karlsruhe d'être nommé parallèlement directeur au centre de recherche de Karlsruhe. Ce cadre a par exemple permis de mettre sur pied en peu de temps un grand institut de recherche sur les nanotechnologies. Des professeurs expérimentés indiquent que, dans les universités, les moyens financiers 
importants, provenant de la DFG ou d'autres sources, sont rarement utilisés de manière concentrée ; ils sont bien souvent répartis entre de nombreux acteurs au sein de l'établissement, ce qui ne permet pas d'atteindre une masse critique suffisante pour financer des projets de recherche significatifs. En dépit de ses avantages pour les deux établissements, la création du KIT donnera sûrement lieu à des négociations laborieuses entre le Bund et les Länder sur leur influence respective et sur les modalités de financement; on peut toutefois s'attendre à un accord couronné de succès.

L'AlLEMAGNe DiSPOSE GLOBALEMENT d'un système d'innovation très perfectionné, dont les structures étaient toutefois jusque récemment celles qui avaient été créées dans les années 1970. Ce constat vaut pour la situation des organismes extra-universitaires comme pour les universités. Le processus de réforme actuel est donc crucial pour que ces institutions s'adaptent aux contraintes d'aujourd'hui et accroissent leurs performances. Mais, comme tous les projets de la réforme n'auront pas les effets escomptés et que des ajustements seront nécessaires, il faut s'attendre à un processus assez long, de l'ordre d'une quinzaine d'années.

Traduction de Marie GRAVEY

\section{Indications bibliographiques}

BUNDESMINISTERIUM FÜR BILDUNG UND FORSCHUNG (BMBF), Forschung und Innovation in Deutschland, BMBF, Berlin/Bonn, 2006

Koschatzky K., Bührer S., Hemer J., Stahlecker T., BJörn W., Die Bedeutung der An-Institute im wirtschaftlichen Innovationsprozess. Studie im Auftrag des BMBF, Fraunhofer ISI, Karlsruhe, 2007

OCDE, OECD Science, Technology and Industry Outlook, OCDE, Paris, 2006

ScHмосн U., Hochschulforschung und Industrieforschung. Perspektiven der Interaktion, Campus Verlag, Francfort/New York, 2003

WiSSENSCHAFTSRAT, Drittmittel und Grundmittel der Hochschulen 1993 bis 1998, Geschäftsstelle des Wissenschaftsrates, Cologne, 2000. 


\section{Eléments du système allemand d'innovation : forces et faiblesses}

\section{Peter WeINGART}

Le concept de système national d'innovation est-il devenu caduc à l'heure de la globalisation? Malgré une tendance croissante à l'harmonisation en Europe, s'inscrivant dans une logique de benchmarking, les différences nationales persistent, comme le souligne Peter Weingart, chercheur à l'Institut pour la recherche scientifique et technique (IWT) de l'Université de Bielefeld. En effet, le système de recherche allemand, qui n'appartient à aucune des deux grandes théories de l'innovation exposées ci-dessous, se distingue notamment du système français dans la nature du rapport existant entre recherche universitaire et extra-universitaire. Dans ce domaine, des questions restent posées outre-Rhin, auxquelles les évaluations menées jusqu'alors, davantage axées sur les organismes que sur le système dans son ensemble, ne peuvent apporter de réponse. Peter Weingart plaide par conséquent en faveur d'une expertise systémique, à même de favoriser une politique globale d'innovation.

Existe-t-il des systèmes nationaux d'innovation ? Seuls la France, l'Allemagne ou d'autres pays sont comparables entre eux. A l'avenir, on peut supposer que les systèmes d'innovation seront de moins en moins nationaux en raison de la mondialisation. En Europe, le processus d'ajustement - le benchmarking par exemple - amène à exporter les modèles par-delà les frontières - souvent sans que l'on sache véritablement ce que l'on « achète ».

Les cultures nationales vont continuer d'influer sur les systèmes de recherche. Ainsi, dans les systèmes nationaux d'innovation, on distingue toujours les rôles que jouent les systèmes de recherche dans différentes missions. Quelle est la place de la recherche militaire par exemple ? Compte tenu de la part considérable de cette dernière dans la recherche française, la France est, en ce domaine, plus proche des Etats-Unis que ne l'est l'Allemagne. Les systèmes d'innovation dont nous parlons ici sont, pour partie, hérités des années 1970 et, pour une autre partie, du $\mathrm{XIX}^{\mathrm{e}}$ siècle. On cite toujours Humboldt, qui joue culturellement un rôle majeur dans le système allemand. Les rapports entre recherche universitaire et recherche extra-universitaire constituent une différence majeure entre la France et l'Allemagne.

\section{Deux grandes théories de l'innovation s'opposent}

L'une est celle que l'on considère comme la succession linéaire de processus de production dans l'innovation; c'est, en quelque sorte, la partition entre recherche fondamentale, recherche appliquée et développement, jusqu'au lancement sur le marché par l'industrie. Ce modèle a des racines au XVII ${ }^{\mathrm{e}}$ 
siècle, et correspond à la prise de distance de la recherche "moderne » par rapport à la politique et à l'Eglise.

On retrouve dans la conception humboldtienne de l'université l'idée de la recherche pure, d'une recherche dont la préoccupation n'est pas le développement industriel mais la découverte des lois de la nature. C'est ainsi, croyait-on, que l'on pourrait produire le plus de créativité. La recherche devait être libre et indépendante des considérations économiques. Cette vision des choses a été reprise après guerre par les Américains; des études récentes montrent toutefois que, dès les années 1920, les industriels américains ont soutenu cette conception selon laquelle ce modèle permettrait de développer de nouvelles idées, qu'ils pourraient reprendre pour les appliquer.

La question est de savoir sur quelle théorie notre système institutionnel de recherche ou de production de savoir repose aujourd'hui. Le paysage institutionnel qui est le nôtre ne se fonde sur aucune espèce de théorie. Il s'est développé historiquement, sur la base de choix aléatoires qui ont mené à ce que les économistes et les sociologues des organisations qualifient de "path dependency». Quand on s'est engagé dans une voie, et que les institutions correspondantes ont été créées, il est très difficile de revenir en arrière. Une remarque subsidiaire à ce propos : c'est aussi dans ce cadre qu'il faut situer l'Initiative d'Excellence visant à restructurer aujourd'hui le système d'enseignement supérieur allemand. On croit pouvoir, avec 1,9 milliard $€$, modifier ce paysage institutionnel. Or, cette mesure peut soutenir les activités quotidiennes, mais elle ne changera pas grand-chose à l'affaire.

Ce modèle linéaire allant de la recherche fondamentale jusqu'au développement a été établi après la guerre par les Américains. Eisenhower qualifiait la recherche fondamentale de « servante de la liberté ». Cette considération est toujours dépourvue de tout fondement théorique; il n'a pas été démontré que les choses fonctionnent réellement ainsi. Le Centre Européen de la Recherche continue de soutenir la recherche fondamentale. On postule que c'est est une activité importante, bien que personne ne sache vraiment pourquoi.

La deuxième théorie, qui s'est développée au début des années 1990 et s'est substituée au modèle linéaire, est celle d'un système national d'innovation. Selon cette nouvelle théorie, il existe plusieurs formes de recherche fondamentale, dont la recherche fondamentale orientée application. Le modèle linéaire existe, mais les modèles circulaire et itératif également. On peut par exemple, dans le cadre d'une recherche appliquée, être amené à découvrir des problèmes fondamentaux qu'il faut résoudre avant de pouvoir parvenir à innover.

\section{Evaluation des systèmes nationaux d'innovation}

Pour évaluer un système, il faut disposer de critères. Alors seulement, on peut dire quels sont les processus optimaux et ce qu'il faudrait mettre en place. La 
théorie des systèmes nationaux d'innovation prenait en compte une multitude de facteurs comme la formation scolaire générale - transmise en quelque sorte au niveau de l'enseignement supérieur - le système fiscal et les organismes de recherche et de développement. Dans le sillage de la réunification, l'idée s'est imposée que l'évaluation doit également s'exercer dans le domaine de la science. Pour le Conseil scientifique (Wissenschaftsrat), qui fut amené à évaluer les institutions extra-universitaires de l'ex-RDA afin de permettre l'unification des deux systèmes, il n'était politiquement plus possible de dire que les autres institutions, à l'Ouest, continueraient de ne pas être évaluées. Par ailleurs, la tendance internationale globale était à l'évaluation, au New Public Management, de sorte que ce processus devenait incontournable pour les organisations scientifiques, quand bien même elles auraient aimé l'éviter. L'ensemble du système de recherche extra-universitaire en Allemagne (la Société Max-Planck, la Communauté Helmholtz, la Communauté Leibniz) est organisé de manière conservatrice et corporatiste. Si l'on considère les résistances apparues dans les années 1990 sur la question de l'évaluation, on sait qu'il s'agit là de défendre des territoires. Cela signifie qu'il n'y a pas de conception globale de l'organisation de l'innovation.

\section{Rapports entre recherche universitaire et extra-universitaire}

Quand on compare l'Allemagne et la France, la première question qui se pose est la suivante : pourquoi faut-il pratiquer une recherche universitaire et une recherche extra-universitaire, et quels devraient être les rapports entre ces deux types d'entités? Dans la tradition allemande, ou tradition humboldtienne, la recherche doit être organisée prioritairement dans les universités. La tradition française fait quant à elle la distinction entre un système d'universités plus large, d'abord dédié à l'enseignement, et un système de recherche, organisé séparément des universités. Intervient maintenant la spécificité allemande, qui est le résultat de causes contingentes. La fondation de la Société KaiserWilhelm, au début du $\mathrm{XX}^{\mathrm{e}}$ siècle, était au fond conçue comme la création d'une institution ayant vocation à organiser le transfert entre les universités et l'industrie. La Société Kaiser-Wilhelm devait mener une recherche tournée vers l'application. Elle regroupait des instituts pour la recherche sidérurgique, pour la recherche sur le cuir, ou encore sur le lait et sur les protéines, des domaines difficiles à placer dans les universités. C'était précisément là la mission de la Société Kaiser-Wilhelm, qui devait par ailleurs être financée par des fonds émanant de l'industrie. Mais la réalité a pris une autre tournure : l'on observe une tendance à l'académisation, de telles organisations ne définissant pas leurs orientations en fonction des mécaniciens ou des ouvriers de l'industrie, mais en fonction de leurs propres communautés de chercheurs. Avec la Société KaiserWilhelm, devenue après guerre la Société Max-Planck, l'un des organismes de recherche extra-universitaires était né. 
La Société Helmholtz est plus récente. Elle a été créée, suivant le modèle américain, à partir des grands centres de recherche nucléaire. Puis l'Etat a fondé d'autres instituts, parfois liés aux "modes» des politiques de recherche. Ont ainsi été créés de grands centres de recherche en biotechnologie ou en informatique. Tous ces organismes devant leur création aux besoins du moment, ils sont ensuite devenus superflus. Ainsi, les centres de recherche nucléaire - après s'être acquittés des missions qui étaient les leurs - ne furent plus nécessaires. Mais on ne ferme pas les grands instituts de ce type; ils ont donc continué d'exister, et ont été regroupés en une communauté d'intérêts. On peut dresser le même constat pour la Communauté Leibniz. Ce type de structure existe parallèlement aux universités allemandes qui déposent par ailleurs plus de brevets que tous les autres organismes. Comme par le passé, les universités allemandes demeurent plus puissantes que toutes ces organisations secondaires.

Si j'étais ministre de la Recherche et que j'avais toute latitude pour prendre des décisions sans devoir écouter qui que ce soit, je fusionnerais les universités et les grands centres de recherche. Nous aurions ainsi les universités de recherche très performantes, à l'américaine, dont notre pays a besoin.

Je trouve également intéressant que l'université allemande soit financée à environ $13 \%$ par l'industrie. L'Etat retire des moyens aux universités, les contraignant ainsi soit à faire appel à la DFG pour obtenir des fonds sur projets soit à rechercher des financements auprès des entreprises. Ce système est bien plus efficace que celui, par exemple, que l'ancien gouvernement de Rhénanie du Nord-Westphalie avait institué : des services de transfert avaient été créés afin d'inciter les universités à pratiquer le transfert de technologie. C'était ignorer que l'Université technique d'Aix-la-Chapelle pratique déjà cette activité depuis un siècle, et avec beaucoup de succès. Cela montre combien il est difficile d'intervenir à l'aide de telles mesures au sein d'institutions comme celle-ci, et qu'il est bien plus efficace de piloter les institutions à l'aide de mécanismes indirects.

Dans le système allemand, la question des relations entre universités et organismes extra-universitaires reste posée. Aucune politique n'a de vision intéressante sur la manière de modifier leurs rapports dans un sens favorable à l'innovation. Les égoïsmes organisationnels qui prévalent dans les différents organismes laissent peu de marge de manœuvre. S'ajoute à cela la complexité des rapports entre Etat fédéral et Länder, qui ne contribue pas à donner une orientation nationale à la politique d'innovation.

Si l'on considère les évaluations déjà réalisées, celle de la Communauté allemande pour la recherche (DFG), celle de la Communauté Helmholtz, celle de la Société Fraunhofer, celle de la Communauté Leibniz - il existe même aujourd'hui aussi une évaluation de la recherche effectuée pour les ministères (Ressortforschung) -, on peut constater, en se référant à la théorie des systèmes nationaux d'innovation, qu'il reste peu de chose des intentions initiales, si ce n'est un appel à instaurer davantage de concurrence. On réclame de l'interdisci- 
plinarité ; on demande que s'installe une flexibilité de l'organisme ainsi qu'une plus grande disposition à coopérer avec d'autres structures. La problématique de la concurrence réside pour partie dans la flexibilisation du droit du travail. Peutêtre l'appel à la Société Max-Planck et à la Communauté Helmholtz, invitées à collaborer plus étroitement avec les universités, constitue-t-il une exception. Les évaluateurs ont constaté de manière unanime que les rapports entre recherche universitaire et recherche extra-universitaire doivent évoluer. Mais cet appel à une coopération plus étroite est pratiquement resté lettre morte.

\section{Une approche systémique est nécessaire}

Les évaluations réalisées se limitaient aux organismes eux-mêmes et ne prenaient pas en considération le système comme un tout. Elles se sont donc concentrées sur des aspects organisationnels. On ne peut donc parler d'une politique globale d'innovation en Allemagne. L'effet le plus positif de l'Initiative d'Excellence a consisté à montrer aux décideurs que l'intervention politique a peu de sens. Le second effet a consisté à susciter des échanges entre des personnes qui n'avaient jusque-là pas communiqué les unes avec les autres. Mais d'autres effets escomptés, une plus grande compétitivité du système universitaire allemand au plan international par exemple, ne peuvent se réaliser alors que les structures de rétribution ont été modifiées, avec une tendance à la baisse du traitement de base qui contrecarre à elle seule une éventuelle compétitivité internationale de la recherche allemande. Nous observons une fuite des cerveaux vers les pays européens voisins et vers les Etats-Unis. Il est par conséquent nécessaire et urgent d'établir une politique d'innovation à plus grande échelle et plus claire, véritablement en mesure de répondre au caractère systémique de la promotion de l'innovation.

\section{Traduction de Marie GRAVEY}





\title{
Le système d'innovation français face au modèle allemand
}

\author{
Jean-Yves MÉRINDOL
}

Le système de recherche et d'enseignement supérieur allemand influence-t-il la définition des politiques françaises d'innovation? Si le modèle humboldtien a fait l'objet de nombreuses analyses à la fin du XIXe siècle de ce côté-ci du Rhin, ce n'est qu'à partir de 1968 que les universités françaises ont allié formation supérieure et recherche. Les divergences binationales sont par ailleurs particulièrement nettes si l'on considère le rôle des régions et des Länder en matière d'enseignement supérieur : tandis que la compétence sur ces questions revient clairement aux Länder outre-Rhin, en France, les décisions incombent à l'Etat central, bien que les régions aient progressivement commencé à intervenir dans ce domaine depuis une quinzaine d'années. Mais par-delà la comparaison franco-allemande, dans le contexte global de valorisation scientifique, il apparaît que c'est le modèle américain d'innovation, accordant une place essentielle à la formation, qui s'impose incontestablement à l'international.

Je ferai un point historique pour ensuite entrer dans les questions d'actualité. Ce qui me frappe, c'est le fait que depuis longtemps, et encore dans les discussions qui se sont menées sur les dernières années en France, la France accorde une extrême importance à l'organisation allemande en matière d'enseignement supérieur et de recherche. Ainsi, des réformes récentes se sont explicitement appuyées sur des exemples allemands : les Instituts Fraunhofer pour les Instituts Carnot, les évolutions de la Max-Planck pour réfléchir aux organismes de recherche en France, l'Initiative d'Excellence, largement débattue en France, inspire certaines propositions de constitutions de grands campus. Plus anciennement, dans l'évolution des IUT, le parallèle avec les Fachhochschulen a été souvent évoqué, les débats sur l'apprentissage dans l'enseignement supérieur se sont toujours référés à l'Allemagne. Les Instituts Steinbeis sont aussi des exemples qui reviennent régulièrement dans les débats français.

Il y a donc, sur le long terme, une attention particulière portée, de manière parfois superficielle mais pas seulement, par les universitaires, les hommes politiques et les décideurs français à la situation allemande. Cette attention est assez largement fondée sur l'idée, d'ailleurs assez bien illustrée dans les tableaux qui nous ont été présentés par nos partenaires allemands, que le système allemand d'innovation constituait un modèle d'organisation cohérent et globalement efficace. Je vais essayer d'expliquer que cette attention est en train de perdre son importance. La deuxième grande référence sur les questions de l'organisation de l'innovation est évidemment, en France, la référence américaine. Et je pense que même si subsiste encore en France la comparaison avec l'Allemagne, de fait c'est la référence américaine qui a de l'importance en Europe 
actuellement et pour l'avenir. J'affirme cela sans nuance, et de façon trop provocatrice, afin de mieux tracer la ligne de mon intervention qui se limitera pour illustrer cette thèse à des exemples français.

\section{La défaite française de 1870. Le modèle allemand : de l'espoir des réformateurs aux décisions des politiques.}

Comment expliquer cette grande sensibilité française à la situation allemande ? C'est bien entendu pour des raisons générales qui tiennent aux relations entre les deux pays, pour des raisons historiques dues à la dernière guerre mondiale puis à la construction européenne, mais aussi, dans le domaine universitaire, à cause du traumatisme qu'a représenté en France la défaite de 1870.

La façon dont la France a analysé sa défaite de 1870 a eu une importance considérable, pendant plus de 100 ans, sur l'organisation de son système universitaire. Les choix d'organisation des universités en France à la fin du $\mathrm{XIX}^{\mathrm{e}}$ siècle, après toutes les complications dues à la Révolution française, sont dans la droite ligne de la défaite de 1870. La France est alors convaincue que l'une des causes de sa défaite réside dans la puissance de la science allemande, on dirait aujourd'hui du modèle d'innovation allemand, et en particulier du rôle qu'y jouent les universités. Le système allemand a été amplement analysé juste après 1870. De nombreuses missions d'étude ont été réalisées, de nombreux réformateurs vont appeler de leurs vœux le transfert en France de ce modèle allemand, construit autour d'universités puissantes et autonomes, alliant formation et recherche. Et les décisions vont suivre pendant près de 30 ans : ce sont autour des lois et des décisions d'investissement prises entre 1880 et 1896 que la France a construit un système qui est resté stable pendant près de 90 ans.

Ce dispositif, qui a tenu sans changements majeurs jusqu'en 1968 et qui continue à influencer notre système actuel, a été organisé en référence au modèle allemand, mais dans une référence qui a, de façon délibérée, choisi de s'en écarter, et même de s'y opposer sur des points essentiels. La décision française, qui n'a pas évité les écueils d'une réaction chauvine, peut se résumer ainsi : le système allemand est puissant, mais le génie français - irréductible à tout autre - nécessite de ne pas s'organiser de la même façon. La conséquence est qu'il a fallu attendre en France 1968 pour se créer des universités qui se rapprochent de structures juridiques de plein exercice, alliant la formation supérieure et la recherche. Jusqu'à cette date, les universités n'ont pas existé, ou seulement de façon éthérée.

L'organisation choisie en 1896, reposant sur des facultés ayant peu de rapports entre elles et très soumises au pouvoir central du ministre de l'Instruction publique et à ses représentants locaux que furent les recteurs d'académie, a été décidée après des débats politiques très clairs qui ont frontalement opposé de grands universitaires-administrateurs qui, très influencés par leur bonne connaissance des universités allemandes, voulaient de 
vraies universités (de grands réformateurs comme Louis Liard y tenaient), et un système délocalisé, représenté par des élus (notamment les sénateurs et les députés) et les grandes villes. Les grandes municipalités provinciales, et leurs défenseurs au parlement, ont refusé le système à l'allemande car elles craignaient de voir la disparition des petites facultés qui existaient ici et là. C'est pour défendre ces situations acquises que l'on a eu recours, de façon plus imagée et plus grandiose, au "génie français ». Le système français qui s'est construit à partir de 1880 et qui a perduré jusqu'en 1968 est un système extrêmement étrange puisqu'il est basé sur un compromis entre deux pensées irréductibles : la volonté absolue de centralisation et d'uniformisation - et le système a été extraordinairement centralisé, infiniment plus qu'en Allemagne -, et la volonté des grands élus locaux de maintenir un dispositif très émietté et peu concentré.

\section{La création des universités en 1968}

Tout ceci a commencé à changer à partir de 1968, particulièrement pendant la période allant de 1968 à la fin des années 1980, marquée par des réformes importantes du système universitaire français. Le point essentiel, décidé après la crise de 1968, est la création des universités. Ainsi, les universités relativement autonomes n'ont en France que 40 ans d'existence, pour les plus anciennes d'entre elles, ce sont des institutions jeunes, qui n'ont ni la même histoire, ni la même façon de se concevoir que les universités allemandes ou les universités américaines. On emploie le même mot, c'est naturel, mais il faut avoir conscience que l'on parle en fait de structures qui ont des histoires, et donc des comportements radicalement différents.

\section{L'émergence du fait régional, donnée de fait sans traduction institutionnelle}

La comparaison France-Allemagne ne peut faire l'impasse sur la comparaison institutionnelle régions/Länder. Les régions en France ont autour de 30 ans : on a, là aussi, un dispositif récent. Les régions françaises n'ont pas officiellement de compétence en matière d'enseignement supérieur et de recherche.

Elles ont peu à peu acquis une compétence, en lien avec les universités et la recherche, qui est celle du développement économique. C'est à partir de cette compétence économique qu'elles sont intervenues, d'abord d'une façon indirecte, et ensuite de plus en plus directement, sur l'enseignement supérieur et la recherche. Elles l'ont fait au début des années 1990, lorsque l'Etat central a décidé, pour la première fois depuis les années 1960, d'efforts financiers importants pour l'immobilier de l'enseignement supérieur et de la recherche publique. Mais il a simultanément décidé que les régions et les autres pouvoirs publics locaux devaient être contributeurs à ces efforts. On assiste alors à la mise en place du plan Université 2000, prolongé en 1998 par le plan U3M, 
mécanismes qui ont créé des conditions de partenariats entre l'Etat et les régions autour des universités. Mais on n'a pas, contrairement à l'Allemagne, un dispositif institutionnel qui répartit des compétences entre les régions et l'Etat central et donne à chacun des deux niveaux des pouvoirs qui peuvent être ensuite source de discussions. On a un dispositif étrange, où la tutelle, sauf rares exceptions, revient à l'Etat central et l'Etat central, ne se sentant pas en capacité, notamment financière, de faire face aux obligations de cette tutelle, a donc demandé et accepté que les régions interviennent. Les régions, après avoir hésité longuement pour certaines, agissent maintenant toutes, mais dans un cadre institutionnel flou et se retrouvent ainsi dans une situation bancale qui n'a pas évolué depuis 15 ans.

\section{Les systèmes d'innovation : nationaux ou locaux?}

Puisque les régions se retrouvent amenées à intervenir sur les questions d'enseignement supérieur et de recherche, et qu'elles le font de plus en plus volontiers (même si certaines d'entre elles, la région Ile-de-France par exemple, ont dans un premier temps refusé), comme elles ont aussi la compétence en termes de développement économique et que les élus, et c'est naturel, sont très sensibles à la thématique de la création d'emplois, on peut se dire que ce sont les régions qui devraient avoir le rôle déterminant et central dans les questions d'innovation. C'est à elles que devrait logiquement revenir la mission de développer les relations entre les dispositifs académiques de production de la recherche et le dispositif de valorisation, notamment via un partenariat renforcé avec les entreprises.

Pourtant ce n'est pas fréquemment le cas en 2007 : il est extrêmement rare que les régions interviennent d'une façon significative et encore moins comme organisatrices de ces dispositifs d'innovation, bien qu'on doive noter des évolutions importantes avec la création de quelques agences régionales de l'innovation. On retrouve là, jusqu'à présent au moins, les conséquences d'une certaine tradition centralisatrice, où les dispositifs d'innovation, quand ils sont mis en place, le sont de deux façons : soit par des mécanismes de soutien ou d'organisation directs de l'Etat central, soit par des systèmes cette fois-ci extrêmement décentralisés à travers les différents établissements publics déconcentrés qui organisent la recherche, qu'il s'agisse des universités, des organismes de recherche, ou d'autres institutions qui jouent un rôle dans ce domaine.

\section{Les organismes de recherche}

Dernier point historique, la genèse et le développement des grands organismes de recherche. On n'a pas eu en France une mise en place des organismes de recherche qui corresponde à une vision aussi rationnelle que celle qui a été présentée ici pour l'Allemagne, avec des organismes de recherche qui seraient 
consacrés à de la recherche fondamentale (Max-Planck), d'autres qui seraient consacrés à la recherche plus liée à l'industrie, à la valorisation (type Fraunhofer), etc.... On a là aussi des décisions en très grande partie basées sur la continuation, avec des petites modifications, de ce qui existait auparavant. Le seul contre-exemple à peu près significatif étant la re-création du CNRS en 1945.

Le CNRS a bénéficié d'une situation inouïe, due en grande partie aux bouleversements apportés par la Seconde Guerre mondiale. Il avait été créé, avant guerre, comme une caisse apportant un soutien financier à la recherche qui se développait dans les facultés. Ce premier CNRS a été créé peu de temps après que l'Etat décide de créer un ministère spécialisé avec, pour la première fois, un secrétariat d'Etat qui s'occupait de la recherche. C'était le moment où le gouvernement issu du front populaire s'est dit : il faut qu'on ait un rôle direct en recherche, c'est-à-dire un rôle d'organisation et de financement. Il a créé cette structure de financement, puis après guerre l'Etat s'est retrouvé face à d'autres graves problèmes, principalement de reconstruction de la France. Le CNRS a alors une mission beaucoup plus ambitieuse, d'organisation de laboratoires propres et de coordination nationale. Mais l'Etat développe immédiatement d'autres structures plus spécialisées, comme le CEA, forcément concurrentes. L'Etat a continué cette politique étrange de conforter un organisme de recherche généraliste, supposé s'occuper de toute la recherche publique, tout en créant et développant des structures de recherche diversifiées et spécialisées à caractère souvent plus industriel et sectoriel.

Le CNRS a vécu jusqu'à la fin des années 1960 dans une relative autarcie vis-à-vis des universités (mais pas des universitaires qui le dirigeaient), puis il a mis en place vers 1965 un dispositif de partenariat avec les universités à travers les unités mixtes, ce qui a profondément changé ce paysage en devenant peu à peu une des normes d'organisation de la recherche de qualité. Mais on n'a jamais eu cette conception allemande d'une organisation rationalisée basée sur une distinction assumée entre recherche appliquée/recherche fondamentale.

De fait, l'Etat a agi au gré des nécessités, en organisant le développement scientifique dans les différents secteurs, et en tenant compte des découpages entre les divers ministères qui cherchaient tous à avoir leurs propres laboratoires ou structures. Ainsi l'INSERM, l'institut national qui a actuellement en charge la recherche médicale, vient d'une structure de recherche liée au ministère de la Santé, travaillant sur l'hygiène et la santé publique. Plus tard, la création de l'INRIA, l'institut de recherches sur l'automatisme et l'informatique, a résulté d'une volonté ministérielle conjointe, sur une thématique précise, des deux ministères (industrie et enseignement supérieur) suivant ainsi une analyse provenant principalement du ministère de l'Industrie. Dernier exemple, l'organisme qu'on appelle aujourd'hui le CIRAD a rassemblé de petites structures techniques de recherche ou de développement qui s'occupaient de 
certaines productions tropicales comme la datte, certaines huiles végétales, d'autres cultures, héritages directs de la colonisation puis de la décolonisation.

Ce détour historique explique au passage l'une des raisons de la difficulté des comparaisons internationales. Ainsi, doit-on considérer que les crédits dont dispose le CNRS sont à prendre en compte pour les comparaisons internationales de la recherche universitaire? La réponse, qui a été négative avant les années 1970, est maintenant plutôt positive, puisque l'essentiel des unités de recherche du CNRS sont maintenant communes avec les universités. Mais il y a quand même beaucoup de moments où on nous rappelle que le CNRS n'est pas dans l'université, qu'il a son indépendance et qu'il entend l'affirmer. Le CNRS est donc à considérer pour les comparaisons financières comme un organisme universitaire, mais pour celles qui relèvent de la science des organisations ou des sciences politiques, surtout comme un organisme extra-universitaire.

\section{Où se fait l'innovation?}

Comme viennent de le dire nos partenaires allemands, on n'est plus du tout, dans la réalité concrète des processus d'innovation, dans une organisation qui relève d'une conception linéaire, qui partirait de la science fondamentale vers la science appliquée et même, plus profondément encore, nous ne sommes plus dans un schéma qui permette d'identifier clairement où est la science fondamentale, où est la science appliquée. Je pense qu'une des raisons des difficultés des modèles qui nous sont présentés, y compris d'une partie du modèle français, et une des raisons du succès du modèle américain, c'est précisément que nous avons besoin d'abandonner les conceptions de l'innovation qui s'organisent autour de l'idée qu'il existe une recherche fondamentale, une recherche appliquée et entre les deux un continuum, à l'intérieur duquel on pourrait se placer et s'organiser d'une façon simple. Le véritable succès d'une politique d'innovation est de savoir faire en sorte que des processus arrivent à vraiment faire circuler des gens, des idées, les produits entre le monde académique et le monde industriel. De ce point de vue, je parle ici de la France : nous n'avons ni des mécanismes, ni des organisations qui tiennent compte de ce fait. Ce qui explique bien des déboires et de mauvais résultats.

En 2007 - la presse française s'en est fait l'écho d'une façon polémique - a été publié un rapport de deux inspections nationales, l'inspection de l'éducation nationale et de la recherche et l'inspection des finances, sur la valorisation de la recherche en France ${ }^{1}$. Je vous conseille de lire ce rapport qui est extrêmement sévère sur la valorisation de la recherche. Il y a bien entendu des choses discutables dans ce rapport, mais le constat d'échec de cette valorisation, c'est-à-dire au sens de la capacité d'un dialogue public-privé et de la capacité dans ce dialogue de créer de la richesse, de créer des emplois, de créer de l'activité, le

\footnotetext{
${ }^{1}$ Rapport sur la valorisation de la recherche, établi sous la direction de Henri Guillaume, Paris, janvier 2007.
} 
constat d'échec me semble évident et démontré. Ensuite, savoir quelles sont les causes de l'échec, savoir si c'est parce que le système industriel est mal organisé, ne sait pas dialoguer ou si c'est du côté académique que se situent les blocages : là on rentre dans un débat plus difficile, mais en tout cas l'échec du dispositif français est patent.

Pour autant, ce qui me semble non dit dans la mise en place des politiques publiques françaises, ce sont les rôles véritables que doivent jouer les structures récemment crées qui marquent, a priori au moins, des évolutions importantes. L'évolution la plus sensible a été la création de l'Agence Nationale de la Recherche (ANR) qui attribue des financements à la recherche publique en France sur programmes. C'est quelque chose d'un peu inclassable si on cherche à le comparer aux dispositifs allemands qui viennent d'être présentés : ce n'est pas analogue à la DFG, car la DFG a un rôle dévolu aux universités alors que l'ANR a un périmètre beaucoup plus général. On n'est pas non plus dans un dispositif tout à fait comparable au dispositif américain de la NSF qui a été aussi pris comme modèle, notamment parce que le dispositif américain de la NSF a une continuité par delà les changements politiques.

Là je fais peut-être un procès d'intention et je m'en excuse car l'ANR est toute récente, et parler de continuité pour une structure qui vient juste d'être créée est certainement prématuré, mais les conditions mêmes de sa création et de sa courte histoire ne garantissent pas encore une forme de relative indépendance vis-à-vis du pouvoir politique. La NSF, qui bien entendu a des comptes à rendre aux pouvoirs politiques sur les objectifs fixés par ces derniers, s'est affirmée comme une administration en général respectée, capable de déployer ses programmes et de le faire d'une façon politiquement indépendante. On verra peu à peu si l'ANR sera, comme je l'espère, plus proche de la NSF que je ne le crains, mais ce n'est pas a priori quelque chose de gagné et d'évident.

\section{La formation et l'innovation}

J'en viens au point central de mon propos : qu'est-ce qui fait la force du modèle américain de l'innovation ? Et en quoi est-il plus performant que le système allemand? Il y a de multiples raisons mais je me contente d'insister sur une, que je crois centrale. Le dispositif américain donne à la formation un rôle tout à fait crucial dans les processus d'innovation. Les universités américaines savent former dans leurs grandes universités, en grand nombre et avec des compétences très larges, des personnes d'origines diverses qui peuvent se retrouver ultérieurement : aussi bien des leaders de la recherche académique, des leaders du monde industriel, des responsables politiques. C'est donc un système qui a regroupé dans les mêmes types d'institutions, les grandes universités de recherche, des jeunes qui se rencontrent pendant leur formation, facilitant des rencontres et des échanges, dans un cadre de plus en plus mondialisé. Les 
grandes universités américaines jouent un rôle important dans les débats politiques américains, elles jouent un rôle essentiel dans la recherche fondamentale et l'innovation, elles jouent un rôle éminent dans les évolutions culturelles et sociales. En bref, elles sont au cœur de l'ensemble de ce qui constitue ce qu'on appelle maintenant l'économie de la connaissance. Je pense que c'est principalement en organisant cette polarisation formation-rechercheinnovation-culture autour des universités et en faisant en sorte que ses décideurs issus de milieux divers, de continents divers, et se destinant à des positions variées se connaissent et développent des capacités de dialogue, que le système américain a la souplesse, la force et la pérennité pour avancer et innover. Et que c'est ainsi qu'il est devenu un modèle incontestable et dominant, éclipsant peu à peu l'intérêt pour d'autres systèmes, dont le système allemand.

En France, la segmentation des différentes structures de formation et de recherche crée une difficulté considérable. En forçant à peine le trait, on sait que l'on a en France de trop nombreuses filières de formation qui sont fermées. Ainsi, dans le domaine de l'énergie, qui est un secteur stratégique de la recherche, ou dans le celui de la recherche militaire, autre secteur stratégique, les chercheurs et les décideurs sont quasiment exclusivement formés dans quatre ou cinq grandes écoles françaises, toutes de petite taille et presque toutes coupées du milieu universitaire. En revanche, en biologie ou en médecine, les chercheurs sont surtout formés dans le système universitaire.

Et la formation des grands leaders des entreprises se fait essentiellement dans quelques grandes écoles d'ingénieurs et de commerce, cinq ou six regroupent presque tous ces futurs leaders, elles aussi à l'écart des universités. Comment à partir de là produire en assez grand nombre des rencontres entre les futurs financiers, chercheurs et ingénieurs qui auront à travailler sur les énergies $\mathrm{du}$ futur, leur conception, leur impact sur le climat, sur l'utilisation de ressources biologiques, sur les impacts de santé publique de ces nouvelles sources d'énergie, sur les modes de financement de ces recherches, sur les coûts de ces énergies ....?

On a ici des cloisonnements profonds, affectant des secteurs industriels entiers et gênant les interactions entre les financiers, les chercheurs, les ingénieurs, les gens du marketing. Ces cloisonnements se mettent en place très tôt, souvent avant 18 ans via le système de très grands lycées élitistes français. Ces cloisonnements dans les formations confortent d'ailleurs d'autres cloisonnements, notamment entre certains organismes de recherche et les universités.

C'EST POUR CETTE RAISON qu'à mon avis il n'y a plus, dans le contexte actuel global de production et de valorisation de la science, qu'un système qui puisse être considéré comme un "modèle » d'organisation efficace de l'enseignement supérieur, de la recherche et de l'innovation et c'est le modèle américain, dans sa complexité. Les universités du monde entier s'y trouvent confrontées, ne serait-ce qu'en constatant que c'est - et de loin - le système le 
plus attractif internationalement, ce qui est un avantage considérable dans un contexte mondialisé qui doit penser la diversité culturelle et sociale. Je pense que les efforts des uns et des autres, s'ils veulent être couronnés de succès, doivent consister à s'en inspirer et à s'en rapprocher. Mais cette prise en compte de l'efficacité américaine doit intégrer les histoires des modèles nationaux, ce qui interdit la pure et simple transposition. C'est à cette condition, et selon une logique d'ouverture, du décloisonnement et de l'interaction des savoirs que l'on saura évoluer, en France et ailleurs, vers un système permettant le développement de l'innovation dans des partenariats entre universités, entreprises et société. 

- II -

LES POLITIQUES DE SOUTIEN À L'INNOVATION 



\section{La stratégie « Hautes Technologies » de l'Allemagne : objectifs et réalisations}

\section{Rainer NÄGELE}

Rainer Nägele, membre du bureau de l'Union de la recherche (Forschungsunion) et directeur du Centre de compétences Management de services à l'Institut Fraunhofer sur l'Economie du travail et l'organisation (IAO) de Stuttgart, présente dans sa contribution les modalités et les différentes étapes de la stratégie "Hautes Technologies " pour l'Allemagne, lancée en 2006 par le gouvernement fédéral. En tant qu'organe consultatif, l'Union de la recherche science-industrie, dont les missions sont évoquées ci-après, accompagne la mise en œuvre de la stratégie, intervenant ainsi en complément du Conseil pour l'innovation et la croissance (Rat für Innovation und Wachstum) instauré par la chancelière Angela Merkel.

\section{Situation de départ}

Si l'on observe la situation actuelle en matière de politique d'innovation, il apparait clairement que la pression de la mondialisation s'exerce non seulement sur la production, mais également, de plus en plus, sur la R\&D. La concurrence entre les sites de R\&D s'intensifie, ce qui implique d'adapter aussi le paysage de la recherche. Dans ce contexte, des facteurs de localisation modernes comme la formation, la présence de clusters de recherche ou la disponibilité de personnel hautement qualifié prennent de l'importance. Il est donc temps d'instaurer des conditions qui nous permettent de continuer à exister face à la concurrence internationale.

La scène internationale évolue elle aussi: le rythme n'est plus dicté exclusivement par l'Europe et par les Etats-Unis. Dans le secteur de la R\&D, l'Asie a fortement rattrapé son retard-citons en particulier le Japon et la Chine - de sorte que la part de l'Allemagne dans le potentiel mondial d'innovation est elle aussi en baisse. L'industrie détient une place cruciale dans le système d'innovation allemand: les dépenses d'innovation sont en effet financées aux deux tiers par les entreprises, l'autre tiers émanant du Bund. Ces dernières années, les entreprises se sont toutefois montrées plus réservées en matière d'investissements de $R \& D$, et nul ne sait aujourd'hui si la reprise économique s'accompagnera d'une augmentation des dépenses des entreprises dans ce domaine.

\section{La stratégie « Hautes Technologies » du gouvernement fédéral}

La stratégie «Hautes Technologies» pour l'Allemagne présentée par le gouvernement fédéral est la première stratégie nationale interministérielle ; elle 
vise à donner à l'Allemagne une position de leader sur les principaux marchés d'avenir.

Trois grandes priorités ont été définies :

- promouvoir l'émergence de marchés pilotes basés sur de nouvelles technologies ;

- développer de nouveaux projets communs à la science et à l'industrie ;

- instaurer des conditions favorisant une application rapide des résultats de la recherche dans des produits et des services.

La mise en œuvre de ces priorités relève des acteurs du système d'innovation : politique, science et industrie.

Le ministère fédéral de l'Education et de la Recherche (BMBF) est l'instance centrale en charge de la coordination de la stratégie « Hautes Technologies ». Ce dispositif global regroupe différentes stratégies spécifiques, dans 17 domaines technologiques et champs d'application au total et 5 domaines transversaux. Chacun de ces domaines a été soumis à une analyse «SWOT», qui a permis de mettre en évidence et d'évaluer les forces, les faiblesses, les opportunités et les risques, afin de définir des objectifs et de procéder à des adaptations ciblées. La stratégie «Hautes Technologies» vise également à faire coopérer plus étroitement la science, l'industrie et la politique. On aimerait en outre parvenir à s'engager davantage pour établir des conditions plus favorables à l'innovation et à instaurer une culture d'innovation plus positive.

En allouant 15 milliards $€$ à la stratégie " Hautes Technologies ", le gouvernement allemand apporte une contribution résolue à la réalisation de l'objectif des $3 \%$ du PIB consacrés à la R\&D, et invite tous les acteurs du système d'innovation à orienter leurs investissements dans ce même sens.

Les stratégies d'innovation de la stratégie " Hautes Technologies »

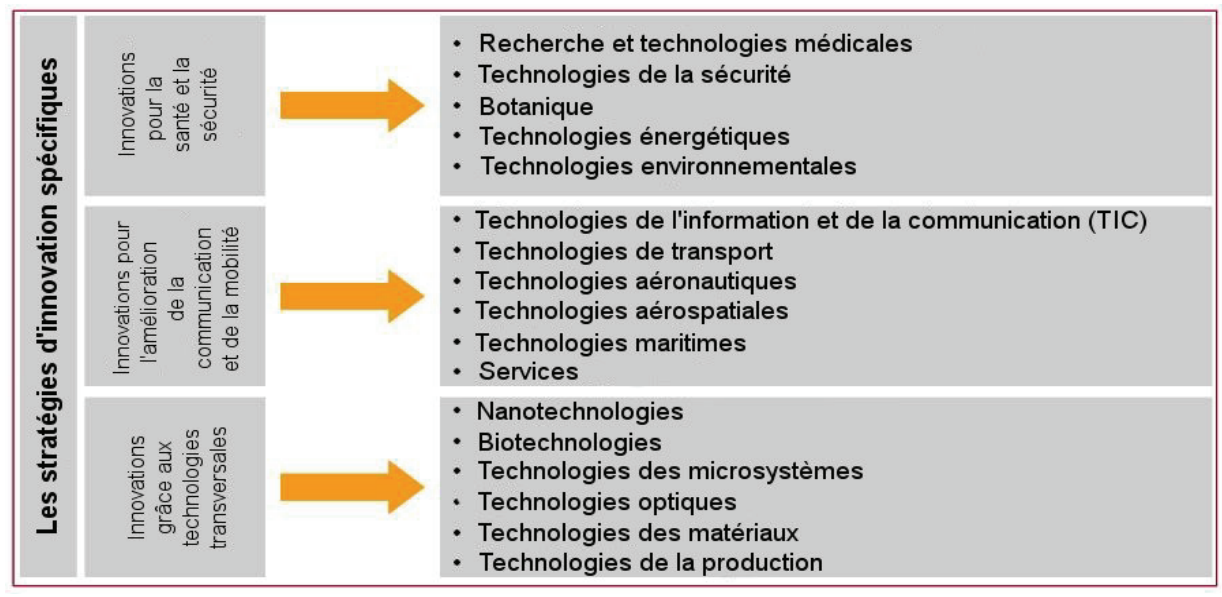

Source : BMBF. 
Comme le montre le tableau, la stratégie « Hautes Technologies » regroupe trois stratégies d'innovation spécifiques : l'innovation grâce aux technologies transversales ; l'innovation pour l'amélioration de la communication et de la mobilité ; enfin, l'innovation pour la santé et la sécurité.

\section{La mise en œuvre de la stratégie « Hautes Technologies »}

Sur les 15 milliards $€$ budgétés, 9 milliards $€$ ont été injectés à ce jour. En annonçant 6 milliards supplémentaires pour la R\&D, le gouvernement fédéral a donné un signal clair. De 2006 à 2009, il est prévu d'investir environ 14,6 milliards $€$ au total dans la stratégie « Hautes Technologies». Sur cette somme, environ 12 milliards $€$ sont alloués aux 17 domaines d'avenir. Ainsi, environ 2,6 milliards $€$ peuvent être consacrés à des mesures essentielles dans le domaine des technologies transversales. Pour la même période, environ 14 milliards $€$ supplémentaires sont engagés pour le financement des institutions et pour le Pacte pour la recherche et l'innovation.

Cette stratégie est organisée sur l'ensemble de la législature. Son avancement fait l'objet d'évaluations régulières. Ainsi, un premier bilan sera dressé en septembre 2007, sous forme de rapport dit «de progrès» (Fortschrittsbericht). A compter de 2008, l'avancement du projet figurera également dans le Rapport fédéral sur la recherche et l'innovation. Les grandes étapes de mise en œuvre de la stratégie " Hautes Technologies» pour l'année 2007 apparaissent de manière synthétique sur le graphique 1.

\section{L'Union de la recherche science-industrie}

L'Union de la recherche (Forschungsunion) science-industrie accompagne la mise en œuvre de la stratégie «Hautes Technologies ». Cet organe consultatif de haut niveau a pour mission d'accompagner la stratégie nationale développée par le gouvernement fédéral. L'Union de la recherche identifie les obstacles à l'innovation, définit les missions de recherche et émet des recommandations d'action concrète à destination des acteurs politiques. Chacun des membres de cet organe est par ailleurs le "promoteur » d'un ou plusieurs des 17 domaines d'innovation et des 5 domaines transversaux de la stratégie «Hautes Technologies ». Chaque promoteur élabore, pour le domaine dont il a la charge, des recommandations destinées au gouvernement fédéral. Le travail de l'Union de la recherche permet d'associer davantage les savoirs et les compétences relatifs à la recherche et à l'innovation. Le dialogue entre la science, l'industrie et la politique contribue à identifier les opportunités de croissance. Ces échanges permettent de créer les conditions pour que les idées débouchent sur davantage de réalisations. Les membres de l'Union de la recherche sont résolument convaincus que l'Allemagne parviendra à s'assurer une position de leader sur les principaux marchés d'avenir. L'Union de la recherche intervient 
donc en complément du Conseil pour l'innovation et la croissance (Rat für Innovation und Wachstum), un autre organe consultatif créé par la chancelière Angela Merkel.

Graphique 1 : Les grandes étapes de la stratégie « Hautes Technologies »

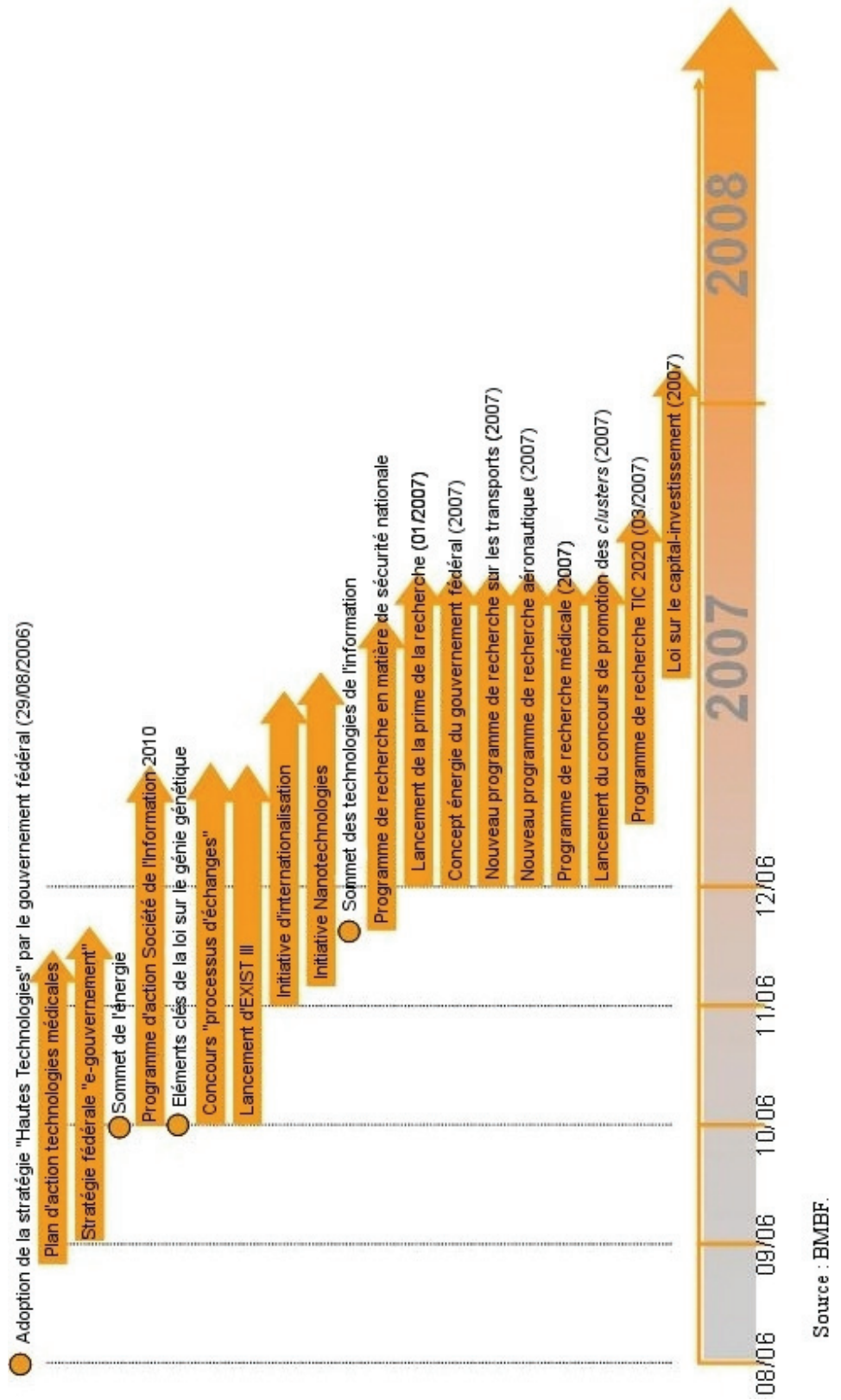


L'Union de la recherche siège six fois par an. Sont inscrits à l'ordre du jour de chaque réunion deux à trois des thèmes de la stratégie "Hautes Technologies »; ces sujets sont étudiés et présentés par les promoteurs concernés. Il incombe alors au promoteur de formuler des recommandations d'action concrètes afin d'appliquer et de faire évoluer la stratégie «Hautes Technologies »; il doit aussi mettre en place des coopérations stratégiques pour traiter son dossier et appliquer les recommandations d'action. Les promoteurs sont donc aussi les «multiplicateurs » d'un domaine d'innovation, à la fois à l'intérieur et à l'extérieur. Outre les directeurs et les membres de l'Union de la recherche, sont également impliqués les responsables des différents services du BMBF ainsi que les secrétaires d'Etat des ministères concernés. Les présentations des promoteurs font l'objet d'une discussion, à la suite de laquelle des recommandations d'action sont définies.

\section{Graphique 2 : Structure de travail de l'Union de la recherche}

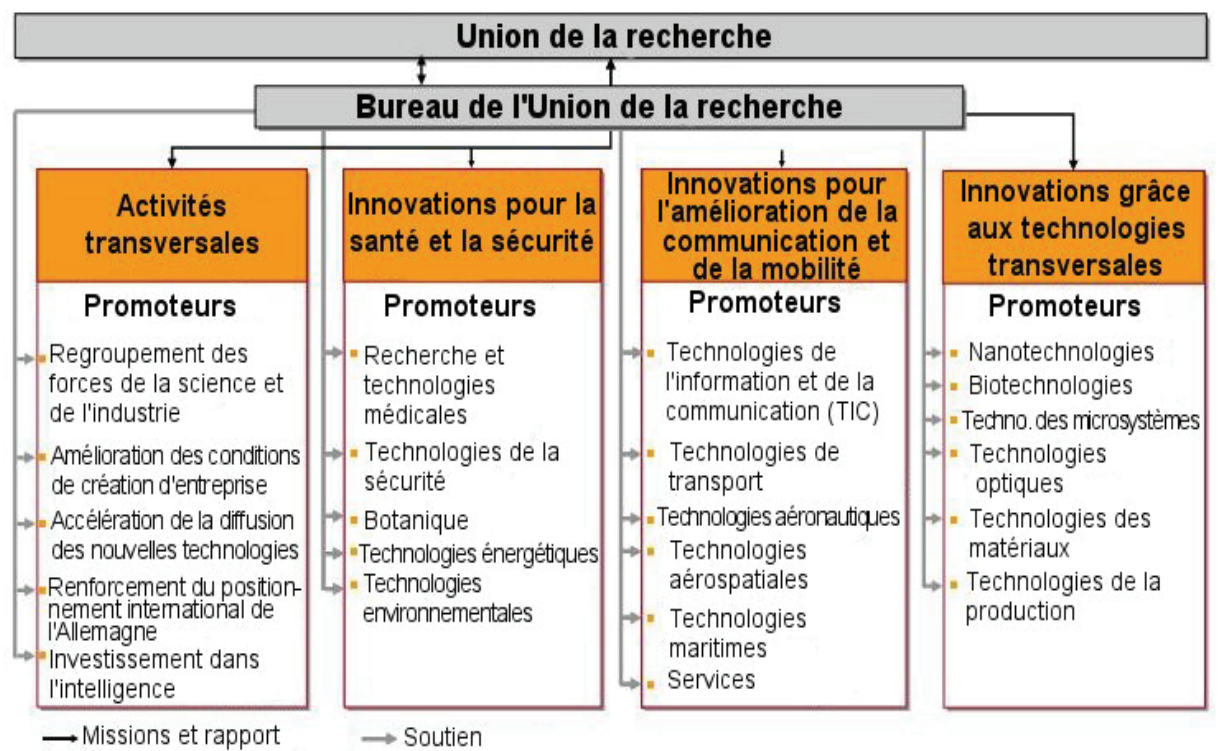

Source : Société Fraunhofer.

Prenons l'exemple de la recherche sur la sécurité. L'objectif est de développer une infrastructure robuste qui fasse de l'Allemagne un site d'implantation attractif. Le promoteur a préparé des recommandations, comme le positionnement de la recherche sur la sécurité dans le contexte national et européen ainsi qu'une ouverture accrue du marché, pour lesquelles il s'agit de proposer des incitations efficaces et de lancer des projets visibles ; ces recom- 
mandations ont été adoptées par l'Union de la recherche. Un dialogue social doit en outre s'ouvrir sur les rapports entre sécurité et libertés civiles.

Le bureau de l'Union de la recherche soutient les promoteurs dans l'élaboration de leurs contributions ; il accompagne le management de la qualité dans son contenu; enfin, il fait suivre les questions reçues de l'extérieur ainsi que les contacts aux promoteurs concernés. Parallèlement, ce service conçoit des évènements à destination du public, réfléchit aux perspectives générales d'évolution thématique et organisationnelle de l'Union de la recherche et suit la mise en œuvre des recommandations d'action.

\section{Situer l'Union de la recherche dans le paysage des initiatives d'innovation actuelles}

On trouve dans le paysage allemand des initiatives d'innovation actuelles le Conseil pour l'innovation et la croissance, l'Union de la recherche et un grand nombre d'initiatives émanant de l'industrie. Si l'on tente de situer ces initiatives selon les dimensions :

- question (politique d'innovation dans une perspective générale ou spécialisée) ;

- $\quad$ orientation (consultative ou applicative) ;

- visée (politique ou économique),

on obtient la représentation suivante :

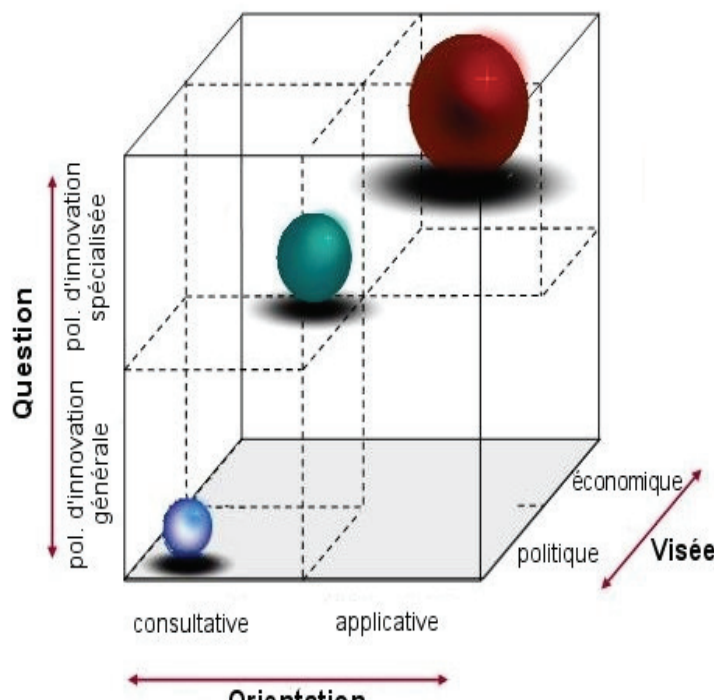
croissance

Union pour la recherche science / industrie

Initiative de la Fédération de l'industrie allemande

La taille des globes illustre la taille de linitiative (nombre de membres)

Source : Société Fraunhofer. 
Suivant cette représentation, le Conseil pour l'innovation et la croissance est un comité consultatif, qui s'adresse aux acteurs politiques et traite des questions générales de politique d'innovation. L'Union de la recherche se positionne à mi-chemin entre conseil et application de questions plus spécifiques de politique d'innovation, l'accent étant mis à la fois sur la politique et sur l'industrie. Prenons pour exemple l'une des nombreuses initiatives émanant des milieux économiques, l'initiative «Stratégies d'innovation et management des savoirs» de la Fédération de l'industrie allemande (BDI). Elle cible des interrogations d'ordre spécifique, avec une forte orientation sur la mise en œuvre par les entreprises. Cet agencement montre à lui seul qu'il n'y aura (?) pour ainsi dire pas d'interface directe entre les initiatives de l'industrie et le Conseil pour l'innovation et la croissance, et que l'Union de la recherche a ici un rôle d'intermédiaire. Les partenariats dits "stratégiques" font partie des moyens destinés à coordonner les initiatives d'orientation plutôt économiques et l'Union de la recherche.

Nous pouvons citer, à titre d'exemple de partenariat stratégique, l'initiative «OLED». Ce programme pour le développement de diodes électroluminescentes organiques doit être soutenu par les pouvoirs publics via le ministère fédéral de l'Education et de la Recherche à hauteur de 100 millions $€$. 33 partenaires issus du monde de l'industrie (dont des PME comme Osram, Merck et Philips) et de la science s'étaient préalablement engagés à réunir euxmêmes 500 millions $€$ pour des activités de $R \& D$ destinées à développer ces diodes.

\section{Exemples de réalisations dans la stratégie « Hautes Technologies »}

La prime de recherche est un exemple de réalisation. Ce dispositif vise à inciter les PME à se tourner vers les activités de R\&D. D'une manière générale, les entreprises sont aujourd'hui davantage enclines que par le passé à collaborer avec des partenaires externes sur des projets de R\&D et à passer des commandes de R\&D à l'extérieur. La part de projets de R\&D d'entreprises menés par des tiers est actuellement de plus de $18 \%$, soit plus de trois fois plus élevée qu'à la fin des années 1970. Mais rares sont les petites entreprises concernées par ces coopérations. Les PME n'investissent actuellement que très peu dans la R\&D. Il leur manque l'argent, mais également le personnel nécessaire à de tels projets. Pour chaque euro investi dans un projet de $R \& D$, les PME reçoivent de 1'Etat $25 \%$ de prime de recherche. Des planchers et des plafonds sont prévus relativement à la taille des entreprises et à l'ampleur des projets éligibles. Le versement aux organismes de recherche mandatés s'effectue après la réalisation et la facturation de la mission.

Le concours de soutien aux clusters d'excellence est un autre exemple de réalisation en Allemagne. Depuis le milieu des années 1990, la promotion de clusters et de réseaux est l'une des priorités de la politique de recherche et 
d'innovation du Bund, des Länder et de l'UE. En construisant sur les acquis, il s'agit maintenant d'étoffer la stratégie de promotion et de définir de nouveaux axes dans le domaine de la constitution de clusters. Comme l'Initiative d'Excellence, exclusivement tournée vers les universités, ce concours vise à identifier les clusters de recherche compétitifs à l'échelle internationale et à les soutenir financièrement. D'ici 2009, deux sessions de concours sélectionneront jusqu'à 10 clusters, auxquels seront alloués 500 millions $€$ au total.

Ces deUX eXemples Ne SONT QUe les PREMIERS RÉSUltats de la stratégie «Hautes Technologies » présentée ici et des travaux de l'Union de la recherche. D'autres travaux prometteurs sont en préparation, et il faut s'attendre à ce que d'autres actions du gouvernement en vue d'améliorer les conditions de l'innovation et d'autres partenariats stratégiques soient mis sur pied à l'automne 2007.

Traduction de Marie GRAVEY 


\title{
La stratégie " Hautes Technologies » de l'Allemagne et la promotion des clusters et réseaux
}

\author{
Sonja R. KIND
}

Bien que communément employés sans distinction, les concepts de "cluster 》 et de "réseau " émanent pourtant de contextes différents : Sonja Kind, conseillère scientifique au VDI/VDE Innovation + Technik GmbH (section socio-économique), en précise ici les principales caractéristiques, après un rappel des objectifs de la stratégie "Hautes Technologies " de l'Allemagne. A l'aide d'études de cas, elle décrit les mesures prises au niveau du Bund et des Länder pour la promotion des réseaux et clusters, destinées notamment à stimuler la coopération entre acteurs économiques et scientifiques.

\section{La stratégie « Hautes Technologies » de la République fédérale allemande}

En août 2006, l'Allemagne a adopté la stratégie «Hautes Technologies », regroupant pour la première fois les activités de recherche et d'innovation, de manière transversale, par-delà les différents domaines politiques et thématiques. Le gouvernement fédéral vise à faire ainsi de l'Allemagne l'un des sites mondiaux compétitifs en matière de science, de recherche et d'innovation. La stratégie « Hautes Technologies » correspond à l'ambition de créer dans le pays un nouveau climat d'innovation. Il s'agit en outre de préserver et de consolider la position allemande sur d'importants marchés d'avenir.

A cette fin, le gouvernement fédéral consacre environ 15 milliards $€$ d'ici 2009 aux technologies de pointe et à des mesures transversales, concernant différentes technologies. En mettant en place cette stratégie et le programme de 6 milliards $€$ lancé en 2006 (porté dans l'intervalle à près de 7 milliards $€$ ), le gouvernement fédéral apporte une contribution considérable à la réalisation de l'objectif défini par l'UE à Lisbonne : porter d'ici 2010 la part des investissements dans la recherche et le développement (R\&D) à $3 \%$ du PIB.

La stratégie «Hautes Technologies » obéit à trois grands objectifs, concrétisés par un grand nombre de nouvelles initiatives en matière de politique de recherche et d'innovation :

- développer des marchés pilotes pour des produits et des services innovants ;

- rapprocher la science et l'industrie afin de commercialiser plus rapidement des produits et des services ;

- rendre les conditions cadre plus favorables pour donner davantage de sécurité et de liberté d'action aux chercheurs et aux innovateurs dans la réalisation de nouvelles idées. 


\section{Schéma 1 : Objectifs de la stratégie « Hautes Technologies »}

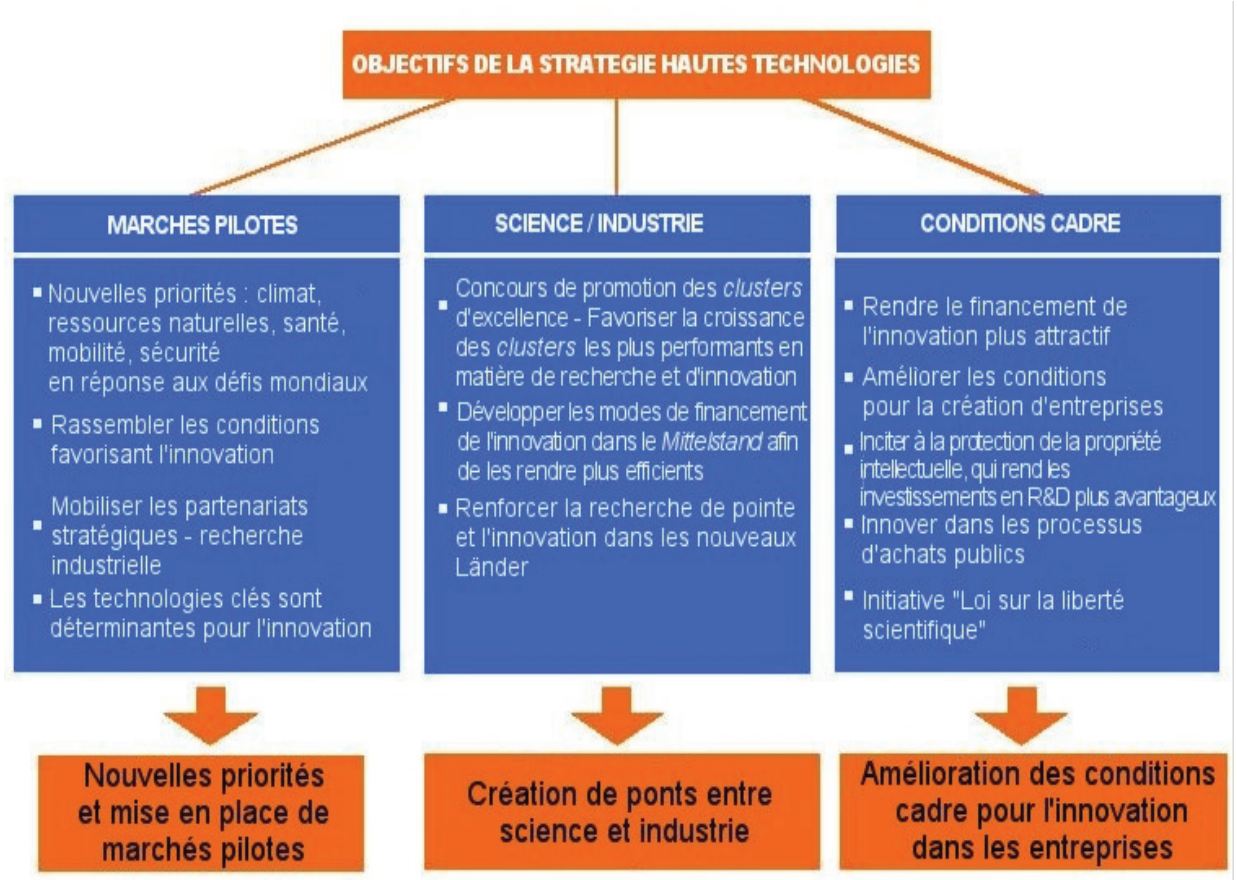

Source : BMBF.

La stratégie « Hautes Technologies » tient compte des défis mondiaux et se concentre sur quatre domaines dans lesquels l'Allemagne dispose déjà d'excellents potentiels économiques et scientifiques : la santé, la protection du climat et des ressources naturelles, la sécurité et la mobilité.

Ce projet s'applique dans 17 domaines d'innovation, les technologies clés créant les conditions préalables à la production de produits et d'applications innovants. Cinq activités transversales étayent en outre la mise en œuvre des objectifs définis. Chacun de ces domaines d'action, avec les mesures qui lui sont propres, vise à simplifier et à écourter le chemin menant de l'idée à l'innovation :

- rassembler les forces de la science et de l'industrie ;

- établir des conditions plus favorables aux créations d'activités et aux PME ;

- $\quad$ soutenir le transfert de technologie ;

- renforcer le positionnement international ;

- investir dans l’intelligence. 


\section{Les concepts de cluster et de réseau}

Il nous faut ici définir et décrire brièvement les concepts de cluster et de réseau, et mettre en évidence leur importance croissante comme instruments de la politique d'innovation. Ces deux concepts émanent initialement de contextes différents. Les réseaux étaient à l'origine des regroupements nés d'une initiative volontariste (créés p. ex. sur intervention politique, par des entreprises ellesmêmes ou par des associations). Ils se caractérisent par l'existence de règles internes, d'engagements contractuels et d'objectifs communs ou bien d'une culture commune. Il n'y a pas nécessairement de concentration géographique, de sorte que les différents acteurs d'un réseau peuvent être dispersés sur plusieurs régions ou pays.

Les clusters émanent quant à eux de « concentrations » régionales d'entreprises préexistant au sein d'un secteur : il y a déjà une masse critique liée à un secteur ou à une chaîne de valeur. Selon Michael Porter, les clusters présentent les caractéristiques suivantes: ils se définissent par une concentration territoriale d'entreprises et d'institutions, et intègrent d'autres organismes publics, semi-publics et scientifiques (universités, Fachhochschulen, chambres de commerce et d'industrie, etc.). Ils ne sont généralement pas orientés sur un secteur, une technologie ou un champ d'application. Un cluster réunit en outre un nombre critique d'acteurs clés entretenant des échanges tout au long de la chaîne de valeur; il s'agit souvent de relations de sous-traitance ou de concurrence, ou bien d'intérêts communs. Une infrastructure permettant une formation initiale et continue de qualité, un accès à l'information et un appui technique est en outre particulièrement importante.

Au fil de l'évolution ultérieure du concept, l'accent s'est porté de plus en plus sur la manière de promouvoir les clusters dans leur développement et de susciter de nouvelles implantations. La Silicon Valley reste un exemple de cluster couronné de succès ; son développement coïncide avec la mise en place, dans les années 1950, d'un pôle de recherche et d'industrie à proximité de la célèbre université Stanford, dans la baie de San Francisco. Au cours des années 1960 et 1970, puis par la suite, de plus en plus d'entreprises ont été créées dans le domaine des technologies informatiques et de l'électronique. Les entreprises les plus célèbres dans ce secteur sont notamment Microsoft, Apple, HewlettPackard ou Google. La Silicon Valley sert de modèle à la plupart des tentatives de créer des clusters de haute technologie regroupant de jeunes entreprises dynamiques.

Contrairement aux réseaux, les clusters ne se dotent pas nécessairement de règles internes ou de liens contractuels. C'est toutefois souvent le cas, si par exemple la coordination du cluster est assurée par une direction en mesure de subordonner les entreprises à des objectifs communs. Si les règles formelles prévalent dans le cas des réseaux, les dépendances entre les acteurs ne sont, de 
loin, pas aussi marquées que dans celui des clusters. Les règles formelles visent souvent prioritairement à renforcer la coopération au sein du réseau. Les réseaux se différencient donc des clusters par des liens comparativement moins étroits puisqu'il n'y a généralement pas encore de tradition de coopération/interaction.

Le critère de différenciation le plus évident entre cluster et réseau est sûrement la plus grande proximité géographique entre les différents acteurs dans le premier cas ; pour le reste, les frontières sont assez floues, et chacun de ces deux concepts peut présenter des caractéristiques de l'un ou l'autre. Ces dernières années en particulier, ces deux notions se sont confondues, de sorte qu'il est de plus en plus difficile de les distinguer. Dans l'usage linguistique général, on emploie d'ailleurs souvent ces deux vocables comme des synonymes.

Schéma 2 : Les frontières entre clusters et réseaux sont floues

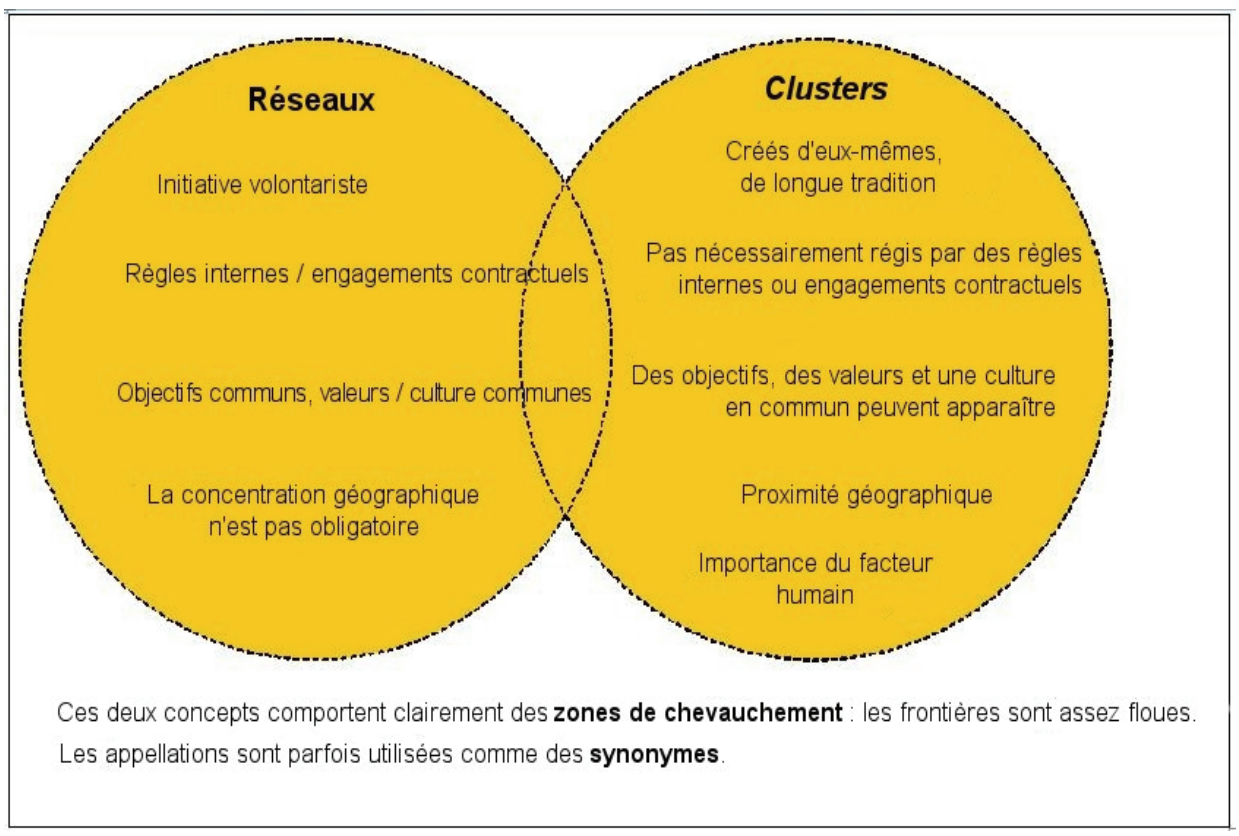

Source : VDI/VDE-IT.

Le financement des clusters et des réseaux détermine la durée des initiatives et donc leur pérennité. On observe généralement un mélange de financement public et privé, la part de financement public prédominant le plus souvent. Les aides publiques sont attribuées par le Bund, le Land et la région, à travers des concours par exemple, comme le concours de promotion des clusters d'excellence dans le cadre de la stratégie « Hautes Technologies». Le soutien à 
l'échelon européen intervient via les fonds structurels européens, notamment INTERREG - «Coopération territoriale européenne» (CTE) (cf. http://de.wikipedia.org/wiki/INTERREG [consulté le 06/09/10]) avec des objectifs de coopération transfrontalière, transnationale et interrégionale.

Après une aide publique initiale, les réseaux et les clusters doivent en règle générale être financés par des moyens privés. Il peut par exemple s'agir de contributions de membres, échelonnées en fonction du chiffre d'affaires et du nombre des salariés des organisations (environ 500 à $3000 €$ ) ou bien de prestations payantes (séminaires, formations, groupes de travail par exemple). La disposition à faire partie d'un cluster ou d'un réseau payant est souvent le reflet de ce qu'apporte cette structure.

On observe globalement une prise en compte croissante du concept « moderne» de cluster et de réseau. La politique de recherche et d'innovation allemande utilise elle aussi de plus en plus ces instruments de promotion, à l'échelon fédéral comme à l'échelon du Land.

Le postulat de base est en effet que les clusters et les réseaux offrent de nombreux avantages. Ils sont supposés permettre un meilleur transfert des savoirs et des technologies de la recherche vers l'industrie, ce qui accélère et améliore la valorisation économique. Les conditions de création d'entreprises semblent elles aussi plus favorables dans ce type d'environnement. Les stratégies de recherche peuvent s'envisager à plus long terme; la mise en place de coopérations internationales est facilitée. Enfin, on espère que les interactions établies au sein de ces environnements pourront assurer un meilleur soutien scientifique et pratique aux futurs chercheurs, et fournir des opportunités d'apprentissage et de formation continue attrayantes. La promotion des clusters et des réseaux vise généralement à accroître l'innovation et à offrir des avantages en matière de compétitivité. Cela peut s'appliquer aux régions, mais ce n'est pas nécessairement le cas.

Les Länder axent eux aussi de plus en plus la politique structurelle régionale sur l'innovation, de sorte que les initiatives de clusters et de réseaux se multiplient.

La part des entreprises qui se trouvaient en 2006 dans un environnement de type cluster (voir carte 1) montre que l'Allemagne et la France avaient encore du retard, alors que l'Angleterre, l'Irlande et la Lettonie étaient leaders en matière de clusters d'entreprises ; l'Italie, l'Autriche et la Bulgarie se trouvaient dans la moyenne haute; la France et l'Allemagne, dans la moyenne basse, tandis que la Pologne, l'Espagne, la Tchéquie et la Grèce étaient les lanternes rouges.

L'Allemagne a réagi à cette situation en incitant à la création de nombreux clusters et réseaux à l'échelle fédérale et régionale. L'une des caractéristiques de la stratégie « Hautes Technologies » consiste à améliorer la coopération entre science et industrie, un domaine où l'approche clusters et réseaux a un rôle à jouer. 


\section{Carte 1 : Part des entreprises opérant dans un environnement de type cluster}

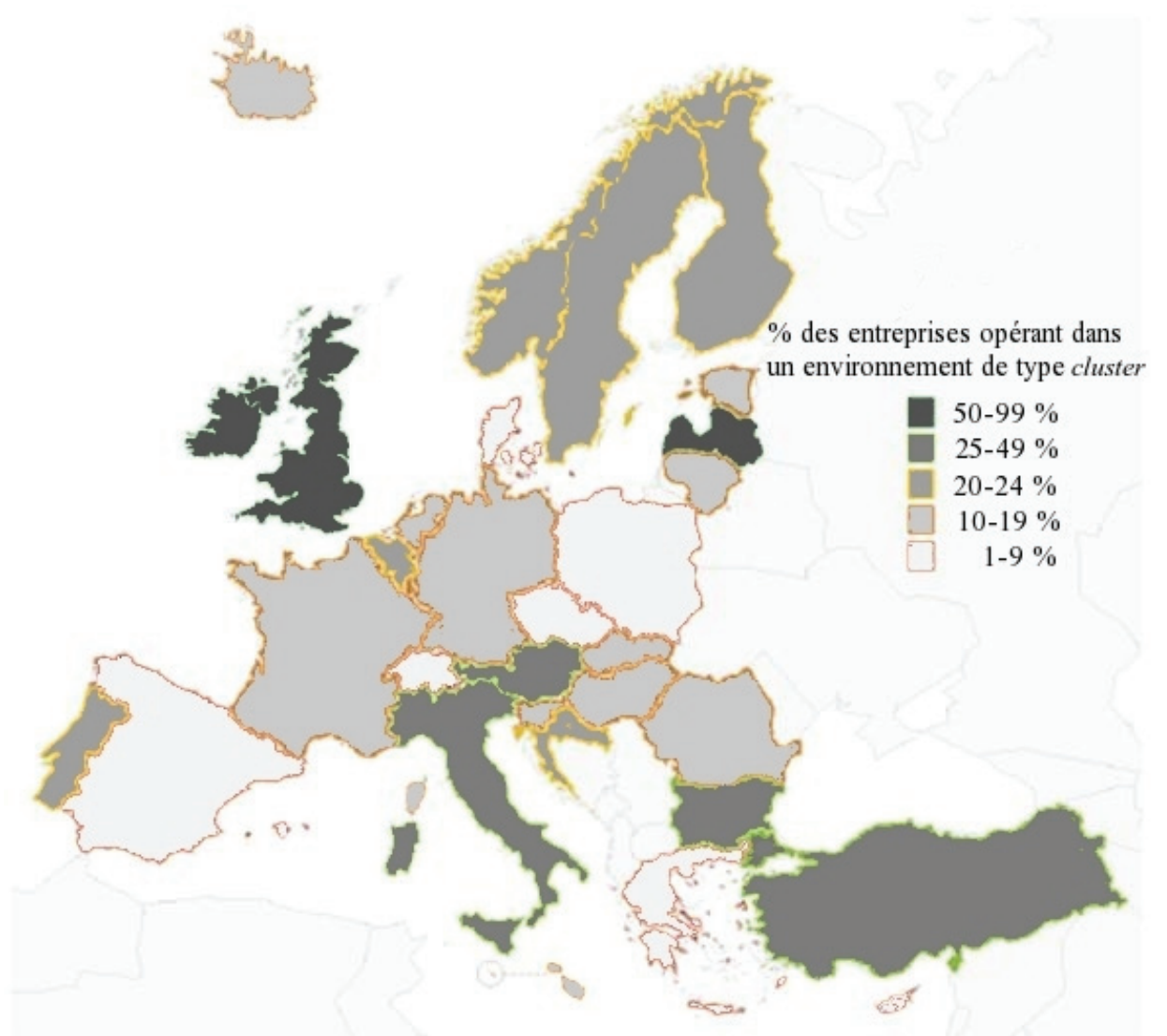

Source : 2006 Innobarometer on cluster's role in facilitating innovation in Europe.

\section{Initiatives de clusters et de réseaux à l'échelle du Bund et des Länder}

Depuis de nombreuses années, l'Allemagne mise sur la promotion des clusters. Le concours BioRegio, lancé en 1995, en constitue un exemple remarquable, qui a contribué à donner le coup d'envoi à l'établissement de l'industrie biotechnologique dans le pays.

Au début de la stratégie "Hautes Technologies », de nombreuses initiatives ont été lancées par le gouvernement fédéral afin de créer davantage d'incitations à la collaboration entre science et industrie et de donner de nouvelles impulsions. Il s'agit surtout de parvenir à valoriser économiquement davantage de nouvelles connaissances scientifiques émanant des universités et des organismes de recherche. L'accent est fortement mis sur le soutien au Mittelstand. 
Nous soulignerons ici les exemples d'initiatives suivants : le concours de promotion des clusters d'excellence, l'initiative d'innovation pour les nouveaux Länder "Unternehmen Region », le concours BioPharma ou les réseaux de compétences.

\section{Schéma 3 : Exemples de mesures publiques pour la promotion des clusters et des réseaux en Allemagne}

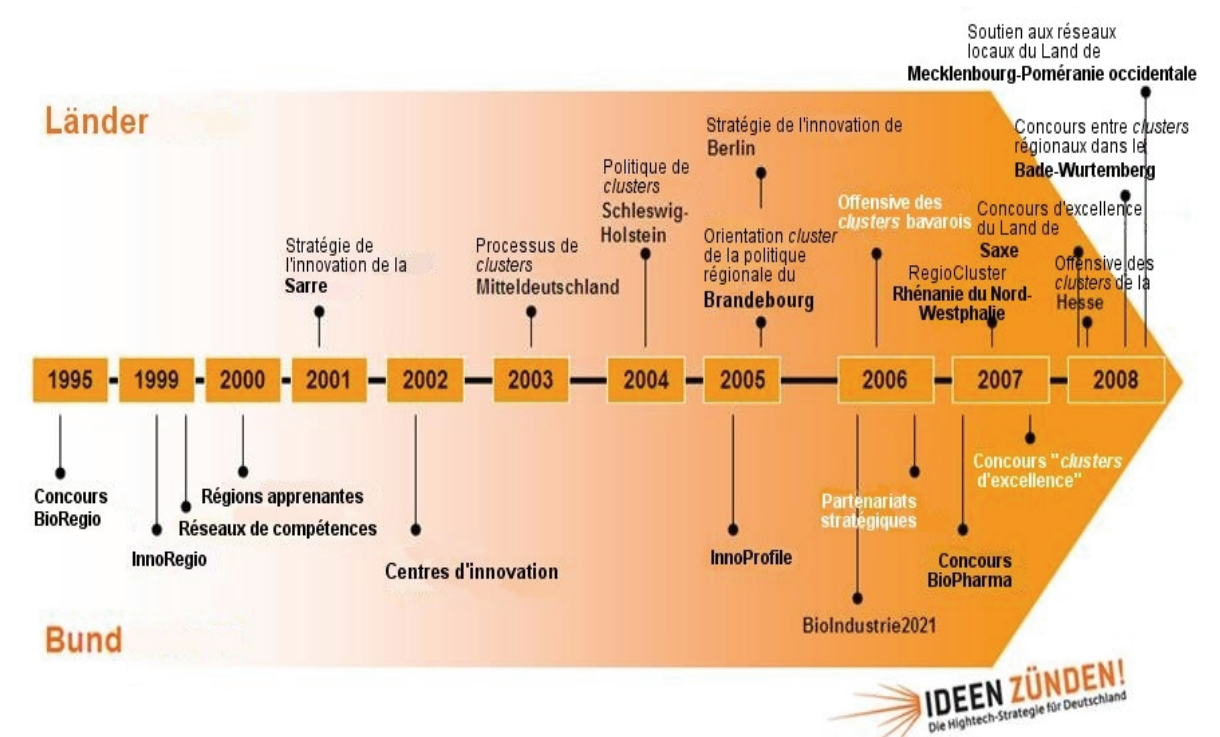

La politique dinnovation allemande est de plus en plus liée aux concepts de clusters et réseaux.

Source : VDI/VDE-IT.

S'ajoutent d'autres initiatives de clusters et de réseaux au niveau régional. Pratiquement tous les Länder allemands ont des activités, individuelles ou communes, dans ce domaine. On constate, là encore, une nette augmentation des initiatives ces dernières années.

Les différentes initiatives des Länder comportent quelques points communs, mais également des différences. Les initiatives se caractérisent par leur portée. Certains mènent leurs propres projets dans tout le Land (Bavière, Rhénanie du Nord-Westphalie, Saxe). Dans certains cas, la mise en réseau fonctionne uniquement à cette échelle, ne se limitant pas à des régions, mais s'arrêtant aux frontières des Länder. Cela signifie que des acteurs se trouvant dans des territoires frontaliers d'autres Länder sont exclus de la participation au cluster. D'autres Länder procèdent autrement. Ainsi, Berlin et le Brandebourg, mais également Hambourg et le Schleswig-Holstein collaborent. 


\section{Carte 2 : Initiatives de clusters et de réseaux des Länder}

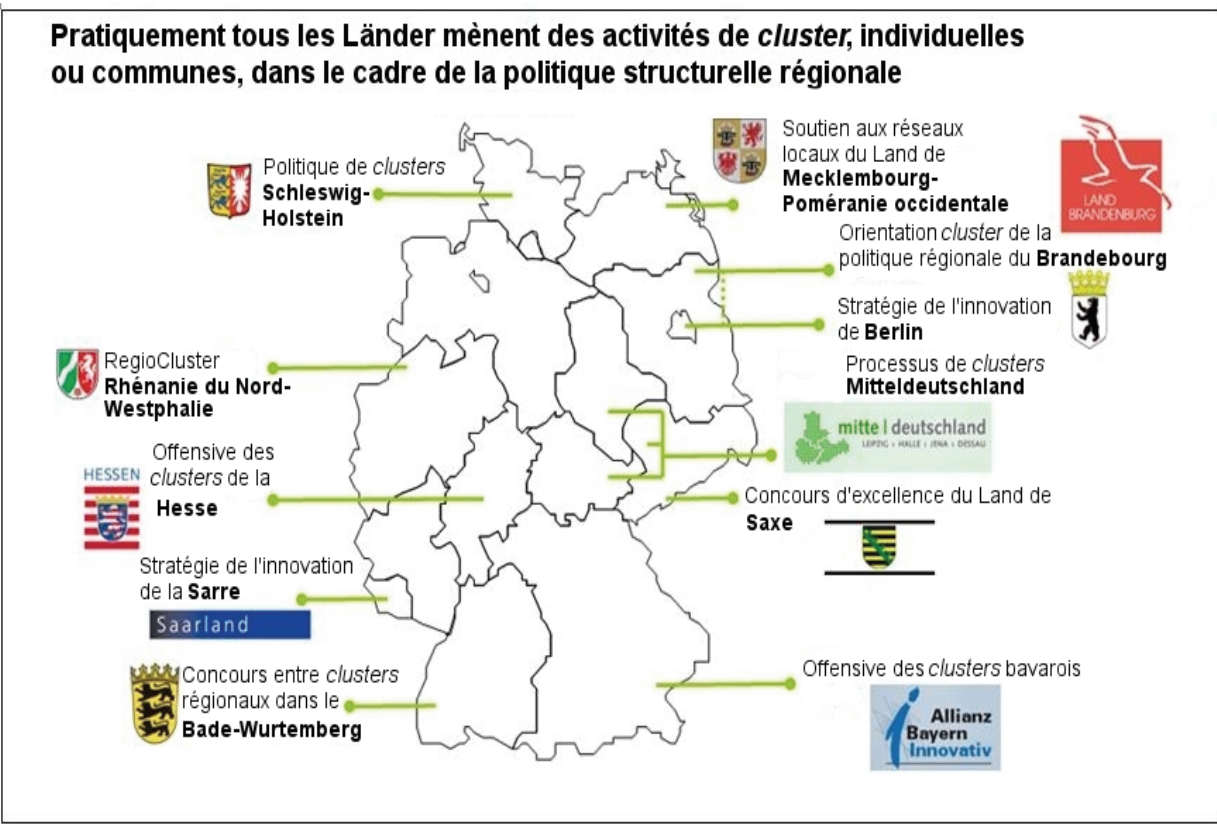

Source : VDI/VDE-IT.

En termes de financement, on trouve surtout un mélange de moyens émanant des Länder et de fonds européens (mission d'intérêt commun - Gemeinschaftsaufgaben, $G A$ - et FEDER). Il arrive exceptionnellement que des initiatives soient financées intégralement sur le budget des Länder. C'est par exemple le cas en Bavière, en Rhénanie du Nord-Westphalie et en Saxe.

La durée des initiatives ne varie guère. Le premier financement est généralement attribué pour trois ans au maximum, le plus souvent sous forme dégressive. À l'issue de cette phase, les clusters et les réseaux doivent être financièrement autonomes. Est parfois prévue une option pour un financement de trois années supplémentaires.

En revanche, le choix des priorités thématiques s'effectue de manière très différente d'un cluster ou réseau à un autre. Rares sont les Länder qui ont fait au préalable des analyses sur les principaux marchés en croissance dans la région (Rhénanie du Nord-Westphalie, Bavière, Saxe).

Les initiatives des Länder se caractérisent aussi par l'évaluation. Pour les mesures soutenues dans le cadre des missions d'intérêt commun (GA), une évaluation est obligatoire au bout de trois ans. Certains Länder tiennent même à intervenir pendant le déroulement des mesures. C'est pourquoi la Bavière, la Rhénanie du Nord-Westphalie et le Schleswig-Holstein mènent une évaluation d'accompagnement. 


\section{Présentation d'exemples}

Nous allons présenter à titre d'exemple quatre initiatives de clusters et de réseaux soutenues soit par le ministère fédéral de l'Education et de la Recherche (BMBF) soit par celui de l'Economie et de la Technologie (BMWT), à l'échelon du Bund ou bien des Länder :

- des partenariats stratégiques, soutenus par le BMBF ;

- le concours de promotion des clusters d'excellence, soutenu par le BMBF ;

- des réseaux de compétences, soutenus par le BMWT ;

- «l'Offensive des clusters bavarois », soutenue par le Land de Bavière.

Partenariats stratégiques

Tableau 1 : Partenariats stratégiques

\begin{tabular}{|l|c|c|}
\hline \multicolumn{1}{|c|}{ Alliances pour l'innovation } & \multicolumn{2}{c|}{ Investissements en millions $€$} \\
\cline { 2 - 3 } & Pouvoirs publics & Industrie \\
\hline Electronique automobile & 100 & 500 \\
\hline Initiative OLED & 100 & 500 \\
\hline Photovoltaïque organique & 60 & 300 \\
\hline Batterie au lithium & $60 *)$ & 360 \\
\hline Imagerie moléculaire & 150 & 750 \\
\hline European Initiative 100 GET & 30 & 225 \\
\hline Mémoire numérique de produit & 30 & 150 \\
\hline $\begin{array}{l}\text { Technologies virtuelles appliquées au } \\
\text { cycle de production }\end{array}$ & 34 & 170 \\
\hline Nanotubes carbone & 40 & 240 \\
\hline *) 15 millions $€$ supplémentaires engagés par la Deutsche Forschungsgemeinschaft et la communauté
\end{tabular}
Helmholtz.

Source : BMBF.

Les partenariats stratégiques et, en particulier, les alliances pour l'innovation constituent une nouvelle approche de la politique de recherche et d'innovation. Ces coopérations stratégiques entre science et industrie ont pour but l'exploitation conjointe des évolutions technologiques prometteuses et des domaines d'innovation, compris comme des marchés pilotes. Les acteurs impliqués couvrent ainsi l'ensemble de la chaîne de valeur. L'une des grandes spécificités 
des alliances pour l'innovation consiste dans l'engagement pris par les entreprises membres au niveau exécutif, permettant d'exercer un fort effet de levier économique. Depuis le lancement de la " Hautes Technologies », la science et l'industrie ont lancé, conjointement avec le BMBF, neuf alliances pour l'innovation. Le Bund apporte 600 millions $€$ de subventions, tandis que l'industrie doit mobiliser près de 3 milliards $€$ d'investissements.

\section{Concours de promotion des clusters d'excellence}

Le concours des clusters d'excellence vise à renforcer la force d'innovation des clusters les plus performants issus de la science et de l'industrie et à les aider à intégrer des groupes d'excellence internationaux.

Un cluster au sens entendu par ce concours est une concentration géographique de différents acteurs en interaction, liés par un même domaine d'activité et dont l'objectif commun est d'accroître leur performance, par la coopération et le regroupement de leurs intérêts et potentiels complémentaires, afin de s'assurer ainsi des avantages en matière de compétitivité à long terme ainsi qu'une position de leader sur le marché. Les acteurs concernés sont notamment des entreprises de l'industrie manufacturière, mais aussi des universités et des organismes de formation et de recherche extra-universitaires ainsi que des structures d'appui comme des sociétés de financement et des organes de l'administration publique.

Le concours se déroule en trois sessions, avec un intervalle pouvant aller jusqu'à un an et demi entre deux sessions. À chaque fois, cinq clusters d'excellence au maximum sont sélectionnés, qui se voient allouer une somme totale pouvant aller jusqu'à 200 millions $€$ sur cinq ans au maximum. La sélection n'obéit pas à des contraintes thématiques; trois critères majeurs doivent toutefois être remplis.

Premièrement, les clusters doivent démontrer qu'ils ont du potentiel. L'évaluation prend en compte l'ensemble de la chaîne d'innovation - depuis l'idée jusqu'à la valorisation économique. Le financement mise sur les forces et sur l'exploitation des potentiels de développement pour accroître la capacité d'innovation, développer les caractéristiques permettant de prendre une position unique sur le marché et d'acquérir ou de consolider une position de leader international. Les clusters doivent en outre avoir une masse critique et une dynamique de développement.

Deuxièmement, le cluster doit avoir une stratégie de mise en œuvre claire et des projets adaptés. Ces projets peuvent par exemple relever des domaines suivants : projets de recherche et développement, soutien aux jeunes chercheurs et qualification, recrutement de personnels qualifiés ou d'encadrement, gouvernance de cluster.

Le troisième critère de sélection est un projet de financement durable convaincant. Il doit être clair que l'industrie et les investisseurs privés 
participent largement et que le cluster sera économiquement viable et pourra fonctionner durablement après l'expiration des aides.

La première session du concours a démarré en août 2007. Les grandes lignes des projets devaient être envoyées en décembre de la même année, puis les douze finalistes devaient déposer leur demande en mars 2008. En septembre 2008, les cinq lauréats du concours étaient annoncés :

- Forum Organic Electronics dans la région métropole Rhin-Neckar ;

- Cool Silicon - Energy Efficiency Innovations from Silicon Saxony ;

- Solarvalley Mitteldeutschland ;

- cluster aéronautique de la région métropole de Hambourg ;

- cluster de biotechnologie "Médecine cellulaire et moléculaire » dans la région métropole Rhin -Neckar (BioRN).

La sélection est réalisée par un jury composé de personnalités indépendantes de haut niveau, présidé par Joachim Milberg, président de l'acatech - Deutsche Akademie der Technikwissenschaften e. V.. Le jury compte 11 autres membres prestigieux issus de la science et de l'industrie. Il est par ailleurs fait appel à des experts pour les questions techniques.

\section{Réseaux de compétences (Kompetenznetze Deutschland)}

Cette initiative, sous l'égide du ministère fédéral de l'Economie et de la Technologie, existe depuis 1999 déjà. Elle a vocation à être le «club des meilleurs réseaux d'innovation» d'Allemagne et à améliorer son positionnement régional et sectoriel. Elle vise également à renforcer la mise en réseau entre industrie et recherche et à promouvoir le développement de clusters ayant une visibilité (internationale). Les réseaux de compétences sont un instrument de soutien à l'innovation, à la croissance économique et à la gestion territoriale.

Ce dispositif s'adresse à un large groupe cible. Ce sont, d'une part, des réseaux régionaux d'innovation, des investisseurs à la recherche de sites et des créateurs d'entreprises ainsi que des décideurs issus des entreprises, de l'administration et de la politique. Sont visés, d'autre part, des scientifiques, jeunes chercheurs, ainsi que les médias et le public intéressé par ces questions. L'adhésion à un réseau de compétences est une sorte de label de qualité : elle n'est pas accessible à tous. La marque «Kompetenznetze Deutschland » a été déposée afin de souligner ce statut de label de qualité. Un comité scientifique de l'agence Kompetenznetze examine, conjointement avec le ministère de l'Economie, les demandes des différents réseaux et valide les adhésions.

L'Allemagne compte aujourd'hui 107 réseaux de compétences dans neuf domaines d'innovation différents. Ces réseaux, implantés dans huit régions, regroupent de nombreux acteurs : plus de 450 grandes entreprises, plus de 6000 
PME et 1600 organismes de recherche, ainsi que plus de 1000 autres prestataires (voir www.kompetenznetze.de [consulté le 06/09/10]).

\section{«Offensive des clusters bavarois »}

L'initiative bavaroise «Allianz Bayern Innovativ» comporte deux piliers: le premier est l'offensive des clusters bavarois (Cluster-Offensive Bayern) et le second, la gestion régionale. Se trouve en toile de fond de cette initiative le constat selon lequel, dans un contexte de globalisation, la concurrence sur les activités et les emplois se durcit dans le monde entier. La Bavière, site coûteux, tente ainsi de relever le défi de la pression d'une concurrence de plus en plus mondiale.

Le premier pilier (l'offensive des clusters bavarois) soutient à l'échelle du Land 19 plateformes de clusters qui s'appuient sur des réseaux et structures de coopération préexistants. Dans certains cas, de nouvelles plateformes ont été créées de toutes pièces. Les plateformes de clusters ont commencé en 2006, avec un horizon de cinq années pour commencer, jusqu'en 2011, et un volume d'aides de 50 millions $€$. Cette politique de clusters se concentre sur l'accroissement de la dynamique d'innovation, la hausse de la productivité et l'amélioration du positionnement et de l'attractivité de la Bavière.

Le second pilier de l'initiative "Allianz Bayern Innovativ» (la gestion régionale) consiste à soutenir les potentiels de développement présents dans les régions, avec l'objectif d'un soutien commun à différentes branches.

Schéma 4 : Offensive des clusters bavarois

\begin{tabular}{|c|c|}
\hline Offensive des clusters bavarois & Trois grands types \\
\hline 19 agences de clusters & $\begin{array}{l}\text { Clusters de haute technologie } \\
\text { Biotechnologies }\end{array}$ \\
\hline Manager du cluster $\begin{array}{c}\text { Porte-parole du } \\
\text { cluster }\end{array}$ & $\begin{array}{l}\text { Navigation par satellite } \\
\text { Technologies de l'information et de la communication } \\
\text { Technologies environnementales } \\
\text { Technologies médicales }\end{array}$ \\
\hline \multirow{4}{*}{$\begin{array}{l}\text { Missions } \\
\text { Mise en réseaux de potentiels } \\
\text { "Rassembler les acteurs autour d'une table" }\end{array}$} & Clusters orientés production \\
\hline & Automobile \\
\hline & Chimie \\
\hline & Capteurs / électronique grand public \\
\hline \multirow{2}{*}{$\begin{array}{l}\text { Prestataire de services pour les entreprises } \\
\text { Constitution et développement d'un réseau de contacts }\end{array}$} & $\begin{array}{l}\text { Alimentation } \\
\text { Bois et sylviculture }\end{array}$ \\
\hline & $\begin{array}{l}\text { Services financiers } \\
\text { Médias }\end{array}$ \\
\hline Entreprises & Technologies énergétiques \\
\hline Universités & Technologies ferroviaires \\
\hline Etablissements de recherche & Logistique \\
\hline \multirow{2}{*}{$\begin{array}{l}\mathrm{CCl} \text {, Chambres des métiers et de l'artisanat, fédérations } \\
\text { Bailleurs de fonds } \\
\text { Institutions de promotion }\end{array}$} & Technologies transversales \\
\hline & Nanotechnologies \\
\hline \multirow[t]{2}{*}{ Conseillers } & $\begin{array}{l}\text { Mecatronique } \\
\text { Automatisation }\end{array}$ \\
\hline & Nouveaux matériaux \\
\hline
\end{tabular}

Source : VDI/VDE-IT, à l'aide de http://www.cluster-bayern.de/ [consulté le 06/09/10]. 
Les 19 plateformes de clusters peuvent se répartir en trois grands types: les clusters de haute technologie, ceux qui sont plutôt orientés production et un troisième groupe tourné vers les technologies transversales. Chaque plateforme est pilotée par un siège pourvu d'un directeur de cluster et représenté à l'extérieur par des personnalités renommées de la science et de l'industrie : les «porte-parole des clusters». Le directeur de cluster s'occupe de mettre les acteurs en réseau et a un statut de prestataire de services.

Les plateformes de clusters occupent une sorte de position intermédiaire entre les clusters et les réseaux, et présentent des traits de ces deux concepts : elles naissent d'une incitation politique, intègrent des acteurs clés, se concentrent sur certains thèmes et ont une visée régionale.

Les initiatives de clusters et de réseaux présentées, menées à l'échelle du Bund ou des Länder, se recoupent parfois. Par exemple, 18 des plateformes bavaroises de clusters sont également représentées dans l'initiative « Kompetenznetze Deutschland».

\section{Rôle du politique dans la promotion des clusters et des réseaux}

Le politique joue un rôle essentiel dans l'orientation des clusters d'entreprises et dans l'organisation de leur impact au-delà des frontières régionales du cluster. Dans le cadre d'une étude consacrée aux clusters d'entreprises et à l'innovation des entreprises allemandes (2006 Innobarometer, 2008 ifo-Schnelldienst), il a été demandé à des entreprises quels paramètres elles considéraient importants pour la promotion des clusters et quels instruments leur semblaient particulièrement adaptés.

Premièrement, en promouvant les clusters et les réseaux, le politique peut contribuer à intensifier l'échange d'informations et la mise en réseau des acteurs industriels, académiques et politiques. L'un des plus importants effets escomptés par les entreprises interrogées est l'augmentation de la main-d'œuvre qualifiée disponible.

L'octroi d'aides financières, directes ou indirectes, est particulièrement important. La mise à disposition d'infrastructures publiques, de bâtiments et d'institutions au sein d'un cluster est perçue comme très importante. Le rôle de la direction du cluster, en matière d'appui aux démarches administratives, d'organisation d'évènements ou de transmission de contacts par exemple, sont des services importants au sein d'un cluster.

Il ne faut pas sous-estimer par ailleurs l'importance du politique dans la réputation et le positionnement de clusters et les mesures stratégiques qui les accompagnent (comme les évènements visant à améliorer la réputation du cluster, la facilitation des relations transnationales). Le politique peut également apporter une contribution importante en matière d'orientation stratégique; il peut influencer jusqu'à la concentration thématique d'un cluster et son impact au-delà de ses limites géographiques. 
Les instruments de soutien devraient se concentrer sur ces critères, afin d'apporter aux acteurs le plus grand bénéfice possible et de promouvoir le développement économique régional et la capacité d'innovation. La promotion des clusters et des réseaux doit toujours tenir compte des données locales. Les clusters et réseaux attractifs attirent la main-d'œuvre, de sorte que les effets positifs se renforcent d'eux-mêmes avec le temps.

POUR CONCLURE, VOICI UNE SYNTHÈSE des principaux aspects pertinents pour les clusters et les réseaux, qu'il convient de prendre en compte dans la mise au point des instruments de soutien, en amont et au cours de la pratique.

Tableau 2 : Critères pour le développement de clusters et de réseaux

\section{Information et communication}

- Base de données détaillée/liste des fournisseurs et prestataires

- Entretiens clients réguliers

- Internet/page d'accueil/lettre d'information

- Evènements réguliers/visites d'entreprises

2. Formation et qualification

- Analyse des besoins de qualification pour le secteur concerné

- Activités de qualification pour les collaborateurs des entreprises

- Evènements réguliers (ateliers, séminaires, etc.)

3. Internationalisation

- Accès à des évènements, thèmes, clients et tendances internationaux

- Soutien de la coopération internationale

- Soutien d'entreprises dans leurs efforts d'internationalisation

- Mise en place d'activités en réseau avec d'autres clusters

- Organisation de visites internationales dans le cluster

4. Développement technologique commun

- Lancement et soutien de projets de coopération

- Etablissement de contacts entre des partenaires de projet potentiels

- Mise en place d'offres de conseil spécifiques

\section{Marketing et RP}

- Supports d'information et marketing

- Constitution d'une identité régionale

- RP nationales et internationales

- Organisation de salons, visites d'entreprises

- Lobbying

Source: 2007 Ossenkopf et. al. Evaluierung und Weiterentwicklung der Netzwerkstrategie des Freistaates Sachsen; VDI/VDE-IT.

Traduction de Marie GRAVEY 


\section{Indications bibliographiques}

COMMISSION EUROPÉENNE, Innovation clusters in Europe: A statistical analysis and overview of current policy support, DG Enterprise and Industry Report, Commission européenne, Bruxelles, 2007

HAFNeR K. A., «Clusterbildung und die Rolle der Politik - wie beurteilen deutsche Unternehmen Firmencluster?», ifo Schnelldienst, 11/2008, 13 juin 2008, p. 37-40

Ossenkopf B., Lo V., Eggers T., Gersten K., Hemer J., Koschatzky K., Wengel J., Feine P., JÜrgens H., WolF B., Evaluierung und Weiterentwicklung der Netzwerkstrategie des Freistaates Sachsen Endbericht für das Sächsische Staatsministerium für Wirtschaft und Arbeit, Fraunhofer-Institut für Systemtechnik und Innovationsforschung, Karlsruhe / Technik. Organisation. Personal.- angewandte Arbeitswissenschaften GmbH, Heidenau, 2004

CENTER FOR STRATEgY AND COMPETITIVENESS (ed), The European cluster memorandum - Promoting European innovation through cluster: An agenda for policy action, European Cluster Observatory, Stockholm, 2007

The GAllup ORganization, Innobarometer on cluster's role in facilitating innovation in Europe, The Gallup Organization [s.1.], 2006.

\section{Liens Internet}

\section{Gouvernement fédéral}

BUNDESMINISTERIUM FÜR BILDUNG UND FORSCHUNG, BioRegio/BioProfile [en ligne]: http://www.bmbf.de/de/962.php [consulté le 07/01/10]

BUNDESMINISTERIUM FÜR BILDUNG UND FORSCHUNG/BUNDESMINISTERIUM FÜR WIRTSCHAFT UND TECHNOLOGIE, Partenariats stratégiques [en ligne] : http://www.ideen-zuenden.de/ [consulté le 07/01/10]

BUNDESMINISTERIUM FÜR BILDUNG UND FORSCHUNG, Concours BioPharma [en ligne]: http://www.bmbf.de/press/2248.php [consulté le 07/01/10]

BUNDESMINISTERIUM FÜR BILDUNG UND FORSCHUNG, Concours de clusters BioIndustrie 2021 [en ligne] : http://www.bmbf.de/de/6955.php [consulté le 07/01/10]

BUNDESMINISTERIUM FÜR BILDUNG UND FORSCHUNG, Initiative «Unternehmen Region » pour les nouveaux Länder [en ligne] : http://www.unternehmen-region.de/ [consulté le 07/01/10]

BUNDESMINISTERIUM FÜR BILDUNG UND FORSCHUNG, Concours "clusters d'excellence » [en ligne]: http://www.spitzencluster.de [consulté le 07/01/10]

BUNDESMINISTERIUM FÜR WIRTSCHAFT UND TECHNOLOGIE, Initiative des réseaux de compétences en Allemagne [en ligne] : http://www.kompetenznetze.de/ [consulté le 07/01/10]

BUNDESMINISTERIUM FÜR WIRTSCHAFT UND TECHNOLOGIE, ZIM - Zentrales Innovationsprogramm Mittelstand, programme de soutien à l'innovation pour les PME [en ligne]: http://www.zim-bmwi.de/ [consulté le 07/01/10].

\section{Länder}

BAYERISCHES STAATSMINISTERIUM FÜR WIRTSCHAFT, INFRASTRUKTUR, VERKEHR UND TECHNOLOGIE, Offensive des clusters bavarois [en ligne] : http://www.cluster-bayern.de [consulté le 07/01/10]

TTN-HESSEN, GeSCHÄFTSSTELlE, Offensive des clusters de la Hesse [en ligne] : http://www.ttn-hessen.de/ [consulté le 07/01/10]

ForschungSZENTRUM JÜlich GMBH, RegioCluster Rhénanie du Nord-Westphalie [en ligne]: http://www.fz-juelich.de/etn/regiocluster/ [consulté le 07/01/10] 
MFG MEDIEN- UND FILMGESELLSCHAFT BADEN-WÜRTTEMBERG MBH, Concours entre clusters régionaux dans le Bade-Wurtemberg [en ligne] : http://www.kreatek.de [consulté le 07/01/10]

WiRTSCHAFTSINITIATIVE FÜR MitTELDEUTSCHLAND GMBH, Initiative de clusters Mitteldeutschland [en ligne] : http://www.mitteldeutschland.com [consulté le 07/01/10]

SAARLAND-MINISTERIUM FÜR WIRTSCHAFT UND WISSENSCHAFT, Stratégie de l'innovation de la Sarrre [en ligne] : http://www.saarland.de/23214.htm [consulté le 07/01/10].

\section{Informations complémentaires}

VDI TeChNologiezentrum GmbH, Kompetenz Cluster, site Internet des clusters et réseaux [en ligne] : http://www.kompetenzcluster.org [consulté le 07/01/10]

BUNDESMINISTERIUM FÜR BILDUNG UND FORSCHUNG, Ideen Zünden, site Internet de la stratégie "Hautes Technologies » [en ligne] : www.hightech-strategie.de [consulté le 07/01/10]. 


\title{
Coordination et harmonisation de la politique d'innovation aux différents échelons politiques. L'exemple de la Saxe
}

\author{
Mark SELLENTHIN
}

Du fait de la structure fédérale de l'Allemagne et conformément au principe de subsidiarité, la politique d'innovation du pays se pratique à la fois à l'échelle des communes, des Länder, du Bund et de l'Union européenne. Dès lors, tout l'art consiste à coordonner les mesures prises à ces différents niveaux décisionnels. S'appuyant sur l'exemple de la Saxe, la contribution de Mark Sellenthin, chercheur jusqu'en 2010 au département d'économie industrielle et de management international du Centre d'études économiques européennes (ZEW), permet de caractériser les mécanismes de coordination et d'harmonisation de la politique mise en œuvre au sein des quatre échelons compétents en matière d'innovation.

\section{Introduction}

La politique d'innovation a pris ces dernières années une importance croissante. L'idée s'est imposée que promouvoir l'innovation et en améliorer les conditions a des répercussions positives sur la croissance économique ${ }^{1}$. Cette contribution vise à décrire la politique d'innovation en Allemagne. La structure fédérale du pays et, par conséquent, la répartition des compétences amènent à la question centrale de la coordination de la politique d'innovation aux différents niveaux décisionnels. Cette coordination sera au centre de notre attention. Nous nous appuyons sur une étude de cas consacrée à la politique d'innovation du Land de Saxe. Ce terme s'entend ici au sens systémique, comme l'ensemble des mesures politiques destinées à soutenir les activités d'innovation. C'est l'interprétation que suivent Lundvall et Borrás (2005, p. 611) : «Cette perspective implique de considérer la plupart des grands domaines politiques à la lumière de leur contribution à l'innovation. L'un des aspects fondamentaux de la politique d'innovation consiste à revoir et à redessiner les liens entre les différentes composantes du système. »

La deuxième partie de ce travail présente les caractéristiques fondamentales de la politique d'innovation en Allemagne. Nous y présentons le système

\footnotetext{
${ }^{1}$ C'est seulement au cours des 30 dernières années que la politique d'innovation est devenue un domaine de la politique économique à part entière. Le rapport de l'OCDE «Changement technique et politique économique », publié en 1980, a constitué une étape importante, qui a contribué à faire de ce domaine un champ politique. La politique d'innovation a pour objectif l'amélioration des performances de l'ensemble d'une économie nationale. La politique scientifique et technologique en est un volet important. La politique scientifique a pour objectif la production de savoirs scientifiques. La politique technologique se concentre sur le soutien et la commercialisation de connaissances technologiques sectorielles et, ainsi, l'amélioration des performances économiques de certains secteurs et branches (voir également Lundvall et Borrás, 2005).
} 
fédéral allemand et les différents niveaux décisionnels en charge de ce domaine. La troisième partie illustre la politique d'innovation à l'échelon régional, à partir de l'exemple de la Saxe. La quatrième partie analyse les mécanismes de coordination de la politique d'innovation dans le système fédéral. Nous terminerons par une courte synthèse.

\section{Politique d'innovation dans un système fédéral : l'Allemagne}

Commençons par présenter brièvement la politique actuelle d'innovation en Allemagne ${ }^{2}$.

Tableau 1 : Politique d'innovation aux différents niveaux décisionnels

\begin{tabular}{|l|l|l|}
\hline \multicolumn{1}{|c|}{$\begin{array}{c}\text { Niveau } \\
\text { décisionnel }\end{array}$} & \multicolumn{1}{c|}{$\begin{array}{c}\text { Porteurs de la politique } \\
\text { d'innovation }\end{array}$} & \multicolumn{1}{c|}{ Missions } \\
\hline Union européenne & Commission européenne & $\begin{array}{l}\text { Politique de cohésion } \\
\text { Politique de R\&D }\end{array}$ \\
\hline Etat fédéral (Bund) & $\begin{array}{l}\text { Ministère fédéral de l'Education } \\
\text { et de la Recherche (BMBF) } \\
\text { Ministère fédéral de l'Economie } \\
\text { et de la Technologie } \\
\text { (BMWi) }\end{array}$ & Politique de R\&D \\
\hline $\begin{array}{l}\text { Echelon régional : } \\
\text { Land de Saxe }\end{array}$ & $\begin{array}{l}\text { Ministère saxon de la Science et } \\
\text { de l'Art (SMWK), } \\
\text { Ministère saxon de l'Economie } \\
\text { et du Travail (SMWA) }\end{array}$ & $\begin{array}{l}\text { Politique de R\&D } \\
\text { Politique de } \\
\text { l'enseignement supérieur } \\
\text { Soutien économique }\end{array}$ \\
\hline $\begin{array}{l}\text { Commune/ville/ } \\
\text { collectivité locale }\end{array}$ & $\begin{array}{l}\text { Service de soutien économique } \\
\text { Solitique de promotion du } \\
\text { site économique } \\
\text { Aide à l'implantation et } \\
\text { aux activités industrielles }\end{array}$ \\
\hline
\end{tabular}

Les porteurs de la politique d'innovation en Allemagne doivent être classés par niveau décisionnel. La politique de soutien à l'innovation se pratique à quatre échelons différents ${ }^{3}$. On trouve généralement au niveau le plus bas le soutien économique des communes; s'ajoute ensuite la politique économique et

\footnotetext{
${ }^{2}$ Pour une présentation détaillée de l'évolution de la politique fédérale en matière de recherche et de technologie, voir Fier et Harhoff (2002).

${ }^{3}$ Notons que la marge de manœuvre financière entre communes et Länder est parfois très variable.
} 
d'innovation menée aux échelons régional et fédéral ; enfin, la politique menée au niveau supranational de l'Union européenne (UE) est de plus en plus importante. Le tableau 1 présente de manière synthétique la politique d'innovation aux différents niveaux décisionnels.

Au niveau des communes, le soutien économique consiste à accompagner les entreprises sur des questions de localisation et à les aider dans leurs projets de développement et d'implantation. Plusieurs programmes fédéraux et régionaux existent en matière d'aide à la création d'entreprises. Cette activité est en outre devenue dans certaines communes un axe essentiel de l'aide économique territoriale, comportant à la fois des prestations de conseil, en particulier en matière d'aides financières et de prévisionnel et, de plus en plus, la mise à disposition de locaux dans des conditions avantageuses pour les jeunes entreprises. De nombreuses communes allemandes ont ainsi aménagé des parcs technologiques et des incubateurs accueillant en particulier de jeunes entreprises high-tech. En outre, les acteurs politiques proposent souvent au niveau local l'intégration à des réseaux utiles ${ }^{4}$.

La politique d'innovation au niveau régional est développée dans la troisième partie, consacrée à l'exemple de la Saxe. Il est extrêmement intéressant d'étudier la politique d'innovation des Länder car ceux-ci ont globalement dans ce domaine les mêmes possibilités d'action que l'Etat fédéral; le volume des dépenses de recherche et de développement est par ailleurs tout à fait important: en 2005, les dépenses de R\&D des Länder représentaient au total 7,7 milliards $€$. Mais les orientations prises diffèrent généralement d'un échelon à un autre. L'Etat fédéral soutient le plus souvent les priorités technologiques du pays et les coopérations nationales, tandis que les Länder apportent généralement leur soutien aux projets d'ampleur financière assez faible, et soutiennent les priorités technologiques régionales. Les gouvernements régionaux étant les porteurs de la politique de l'enseignement supérieur et de l'éducation, le financement des établissements d'enseignement supérieur est fondamentalement du ressort des Länder. Les ministères fédéraux concernés et la Communauté allemande pour la recherche (DFG) assurent un soutien global. Il faut évoquer à ce propos le Pacte pour l'enseignement supérieur, signé par le Bund et par les Länder afin de créer davantage de places dans les universités : 90000 étudiants supplémentaires doivent être accueillis entre 2007 et 2010. L'Etat fédéral investit 565 millions $€$ dans ce pacte, et les Länder assurent le financement global. Dans le cadre du Pacte pour l'enseignement supérieur également, l'Etat fédéral soutient la recherche, via la DFG, à hauteur de 700 millions $€$ d'ici 2010. L'Initiative d'Excellence constitue une autre initiative commune au Bund et aux Länder pour soutenir la recherche universitaire de pointe. Ce dispositif subventionne des écoles doctorales, des clusters d'excellence et des projets d'avenir émanant d'universités allemandes,

\footnotetext{
${ }^{4}$ Voir Sellenthin (2006) sur l'analyse de l'aide à la création d'entreprises à Heidelberg.
} 
avec un budget total de 1,9 milliard $€$, dont $75 \%$ sont financés par l'Etat fédéral ${ }^{5}$. L'Initiative d'Excellence vise à soutenir une recherche académique compétitive, et donc visible, à l'échelle internationale. Cette initiative encourage la concurrence entre les institutions et l'affirmation sur la scène internationale. De nouvelles coopérations se sont en outre établies entre les universités, les entreprises et les organismes de recherche extra-universitaires. En 2005, le Pacte pour la recherche et l'innovation a été lancé afin de renforcer et d'améliorer la recherche dans les structures extra-universitaires. Dans ce cadre, l'Etat fédéral et les Länder mettent des moyens supplémentaires à la disposition des grandes organisations scientifiques que sont la Communauté Helmholtz, la Société Max-Planck, la Société Fraunhofer, la Communauté Leibniz et la DFG'.

A l'échelon national, les grandes orientations données à la politique d'innovation relèvent surtout du ministère fédéral de l'Education et de la Recherche (BMBF) et du ministère fédéral de l'Economie et de la Technologie (BMWi). La mise en œuvre technique et organisationnelle du financement des projets est généralement assumée par des porteurs de projet issus d'institutions de recherche et d'autres organismes. En Allemagne, le soutien à la technologie n'est pas centralisé ; il relève de différents ministères. La priorité du BMWi consiste à soutenir les petites et moyennes entreprises (PME) et les technologies presque mûres pour le marché, tandis que le BMBF se concentre plutôt sur la recherche fondamentale, le système scientifique, et la promotion de la coopération entre science et industrie. Ces deux ministères financent des programmes de R\&D à un niveau considérable. Pour l'année 2008, le budget global du BMBF a été estimé à 9,3 milliards $€$, dont environ 3 milliards pour le

\footnotetext{
${ }^{5}$ Selon le ministère fédéral de l'Education et de la Recherche, les écoles doctorales offrent des programmes de doctorat structurés dans un excellent environnement de recherche et dans un large domaine scientifique. Au total, 39 écoles doctorales reçoivent environ 1 million $€$ par an. Dans le cadre des clusters d'excellence, les universités doivent coopérer avec les organismes de recherche extra-universitaires, les Fachhochschulen et les entreprises. 37 clusters bénéficient d'aides, pour une enveloppe totale de 195 millions $€$. Sont par ailleurs soutenus des projets d'avenir visant à renforcer la recherche académique de pointe. Cette initiative doit également permettre à 9 universités de renforcer leur profil de recherche. Ce secteur est soutenu à hauteur de 210 millions $€$ par an.

${ }^{6}$ Le paysage de la recherche allemand est très différencié. La recherche et l'enseignement se pratiquent dans les universités et les Fachhochschulen. La spécificité des universités est d'offrir un large éventail thématique et méthodologique, tandis que les Fachhochschulen sont dédiées à la fois à l'enseignement, qui est leur mission principale, et à la recherche appliquée. La recherche extra-universitaire est très présente en Allemagne. 4 organismes de recherche se sont établis, avec des caractéristiques bien différentes. Les instituts Max-Planck (MPG) se consacrent surtout à la recherche fondamentale dans les secteurs de la biologie et de la médecine, de la physique, de la chimie et de la technique et dans celui des sciences humaines. Les instituts Fraunhofer (FhG) sont tournés vers la recherche appliquée et la recherche contractuelle pour l'industrie, les entreprises de services et les structures publiques. L'Allemagne compte 15 grands pôles de recherche dotés d'équipements lourds à la disposition de groupes de recherche nationaux et internationaux regroupés au sein de la Communauté Helmholtz (HGF). Les instituts Leibniz (WGL) sont dédiés à une recherche interdisciplinaire axée sur la demande. Les Länder de l'Est disposent par ailleurs des instituts de recherche Forschungs-GmbH, issus des combinats et des instituts académiques de la RDA, qui ne reçoivent souvent aucune dotation de l'Etat fédéral ou du Land.
} 
financement de projets et 3,6 milliards pour le soutien institutionnel ${ }^{7}$. Pour 2008, le budget du BMWi représente 6,2 milliards $€$, dont 563 millions sont alloués à la R\&D et à l'innovation dans les PME, 644 millions au soutien de l'économie régionale et 997 millions à la recherche aéronautique et spatiale (Centre allemand pour la recherche aéronautique et spatiale - DLR - inclus). Le soutien régional à l'industrie joue un rôle particulier. Le BMWi prévoit 644 millions $€$ pour le soutien aux investissements dans le cadre de la mission commune pour l'« Amélioration de la structure économique régionale ». Si l'on ajoute le nécessaire cofinancement des Länder, environ 1,2 milliard $€$ sont mis à disposition. Ces moyens sont destinés à soutenir les investissements des entreprises dans les régions confrontées à des problèmes structurels et à améliorer les infrastructures territoriales de proximité. Le financement se concentre prioritairement sur les nouveaux Länder et sur Berlin. La mission commune BundLänder est un instrument important de la politique régionale en Allemagne et sert à coordonner cette politique. Chaque année, l'Etat fédéral et les Länder définissent un plan-cadre, qui comporte les règles de financement, les taux, les zones concernées et les programmes de financement régionaux des Länder. A l'intérieur de ce cadre arrêté d'un commun accord, chaque Land peut fixer ses propres priorités géographiques et objectives.

Outre l'amélioration des conditions scientifiques et économiques des activités de R\&D et l'accroissement de la compétitivité des entreprises allemandes, la politique allemande d'innovation s'est axée ces dernières années sur l'intensification et l'accélération du transfert de savoir et de technologie. En plus des mesures classiques, comme le soutien à la recherche fondamentale, l'idée de réseau se répand de plus en plus, consistant à promouvoir les coopérations entre les différents acteurs du système d'innovation. Des instruments concrets (InnoNet, InnoRegio par exemple) permettent d'encourager les coopérations. Cette vision de la politique d'innovation, qui mise sur le principe de réseau, peut être qualifiée de «nouvelle approche systémique» (Fier \& Harhoff, 2002, p. 280). Cette perspective tient également compte du financement de systèmes d'innovation régionaux et sectoriels. A travers plusieurs concours, l'Etat fédéral soutient la mise en réseau d'entreprises et d'organismes de recherche, afin de regrouper les forces de régions entières (BioRegio, InnoRegio par exemple).

Avec la stratégie «Hautes Technologies », l'Allemagne dispose pour la première fois, depuis 2006, d'une stratégie nationale pour la politique d'innovation; ce dispositif coordonne les mesures politiques nationales, pardelà les différents niveaux de compétences. Il faut toutefois noter que cette coordination ne s'exerce qu'horizontalement, entre les différents ministères

\footnotetext{
${ }^{7}$ Le soutien de projets permet de financer notamment l'innovation par les nouvelles technologies, l'innovation par les sciences de la vie et l'Initiative d'Excellence pour la recherche de pointe dans les établissements d'enseignement supérieur. Le soutien institutionnel comprend le soutien aux organismes de recherche extrauniversitaires comme la Société Max-Planck et la Société Fraunhofer.
} 
fédéraux, et non verticalement, entre le Bund et les Länder. La stratégie « Hautes Technologies » se focalise sur le soutien du transfert de savoir et de technologie des institutions publiques de recherche vers les entreprises, afin de développer la valorisation commerciale des résultats de la recherche scientifique. Outre le soutien à la recherche d'excellence, cette stratégie vise à créer des marchés pilotes dans 17 secteurs d'avenir. Elle accorde aussi une attention particulière aux PME.

Les banques publiques de soutien sont des acteurs importants de la politique d'innovation en Allemagne. L'établissement de crédit KfW et les banques de soutien des différents Länder proposent aux entreprises, et en particulier aux PME, des crédits à faibles taux d'intérêts et des programmes de financement. En Saxe, c'est la SAB (Sächsische Aufbaubank) qui met en œuvre et administre les programmes de financement du Land ${ }^{8}$.

Au niveau européen, la politique de recherche joue un rôle important. Les programmes de soutien de l'UE, en particulier les programmes cadre pour la recherche, visent à consolider l'Espace de recherche européen (Commission européenne, 2007b). Le $6^{\mathrm{e}}$ programme cadre pour la recherche (2003-2006) a apporté un soutien de 17,5 milliards $€$ à la recherche européenne. Le $7^{\mathrm{e}}$ programme cadre pour la recherche (2007-2013) dispose d'un portefeuille de 50,5 milliards $€$. L'accent est mis sur le financement de la recherche coopérative $(32,4$ milliards $€)$ dans les secteurs suivants :

- santé ;

- agroalimentaire, agriculture et biotechnologies ;

- technologies de l'information et de la communication ;

- nanosciences, nanotechnologies, matériaux et nouvelles technologies de production ;

- énergie ;

- environnement (changement climatique inclus);

- transports (aéronautique incluse);

- sciences humaines et sociales, sciences économiques ;

- sécurité ;

- aérospatiale.

L'UE soutient surtout des consortiums de recherche dont les membres émanent des universités, des institutions de recherche extra-universitaires et des entreprises. Cette politique vise à encourager le transfert de savoir et de technologie. Diverses mesures de financement contribuent en outre à la mobilité des chercheurs (actions Marie Curie par exemple).

Dans le cadre de la politique de cohésion, l'UE se focalise davantage sur la promotion de l'innovation. Pour la période 2007-2013, les fonds structurels européens apportent 26,3 milliards $€$ à l'Allemagne. Ce soutien bénéficie surtout

\footnotetext{
${ }^{8}$ La Banque européenne d'investissement (BEI), banque de développement de l'Union européenne, propose également des prêts à des conditions avantageuses.
} 
aux nouveaux Länder et à Berlin. La réforme des fonds structurels européens pour la période 2007-2013 a établi une corrélation claire entre les objectifs de la stratégie de Lisbonne et les directives de la politique de cohésion : il s'agit de soutenir la recherche, le développement et l'innovation afin d'accroître la compétitivité de l'économie européenne. La politique de cohésion finançait par le passé des projets d'infrastructures visant à améliorer les voies de circulation et de transport. L'utilisation des fonds structurels doit clairement satisfaire aux objectifs de Lisbonne: dans les 15 Etats membres de l'UE, $60 \%$ des dépenses allouées à l'objectif de «convergence» et $75 \%$ des dépenses allouées à l'objectif de « compétitivité régionale et emploi » doivent contribuer à mettre en œuvre la stratégie de Lisbonne. Le "ciblage » (Earmarking) doit permettre de contrôler, mesurer et tracer la cohérence de l'utilisation des fonds structurels par rapport aux objectifs de Lisbonne. Cette règle de ciblage ne s'applique toutefois qu'aux 15 Etats membres de l'UE et non aux nouveaux entrants. Le programme cadre national stratégique pour l'Allemagne (NSRP) va encore plus loin en établissant que $71 \%$ des moyens déployés pour l'objectif de convergence et $81 \%$ des moyens déployés pour l'objectif de compétitivité régionale et d'emploi seront consacrés à atteindre les objectifs de Lisbonne. L'UE contribue ainsi dans une large mesure à la politique d'innovation à l'échelon régional, sur lequel nous allons maintenant nous concentrer.

\section{Politique d'innovation à l'échelon régional : l'exemple de la Saxe}

Pour mieux comprendre les mécanismes de coordination des différents niveaux décisionnels, il est indispensable d'analyser plus précisément la politique concrète d'un Land. La Saxe présente un intérêt particulier en matière d'innovation car elle a très bien su maîtriser les changements structurels et le processus de transition qui a suivi la réunification de l'Allemagne. Cet exemple montre toute l'importance de la coordination et de l'harmonisation des différents niveaux décisionnels. S'ajoute à cela le fait que la Saxe a fortement misé sur le soutien à la haute technologie, ce que l'on peut considérer comme un important moteur de croissance. Cette étude de cas s'appuie sur une analyse de documents et sur des entretiens avec des représentants du ministère saxon de l'Economie et du travail (SMWA) ainsi qu'avec des représentants de la SAB.

La Saxe (4,2 millions d'habitants) est le Land de l'ex-RDA où l'innovation et le développement technologique sont le plus prospères. C'est un pôle de recherche fort. La part des dépenses de R\&D dans le PIB y est de 2,2\%, soit la part la plus élevée de tous les Länder de l'Est ; ce chiffre est toutefois inférieur à la moyenne du pays (2,4\%). Dans les trois plus grandes villes de Saxe (Leipzig, Dresde et Chemnitz), les taux de croissance de la part de la population active employée dans le secteur scientifique sont parmi les plus élevés d'Europe (voir graph. 1). La Saxe a réussi à s'affirmer comme un fort pôle de R\&D. Ce Land compte 4 universités publiques, l'Institut international supérieur de Zittau, 
5 Fachhochschulen, 8 écoles supérieures privées et de très nombreux organismes de recherche extra-universitaires (dont 15 instituts Fraunhofer, 6 instituts Max-Planck, 7 instituts Leibniz et un institut Helmholtz). L'économie saxonne emploie environ 9800 personnes dans la R\&D, et investit chaque année environ 600 millions $€$ dans ce secteur. Environ $60 \%$ des chercheurs employés par l'industrie travaillent dans des PME. Entre 2001 et 2006, les dépenses de R\&D des entreprises saxonnes ont augmenté de 48,9\%.

En Allemagne, l'éducation et l'enseignement supérieur relèvent de la compétence des Länder. En 2006, les établissements d'enseignement supérieur saxons ont reçu du Land une enveloppe de 919,2 millions $€$. En plus de la recherche fondamentale et appliquée, la Saxe encourage tout particulièrement les associations et les réseaux de recherche. Une association de recherche sur les matériaux a été créée à Dresde. L'association de recherche Public Health ainsi que des centres pour la médecine de l'environnement, l'épidémiologie et la biotechnologie environnementales sont implantés à Leipzig. Un centre consacré à la microtechnique a été fondé à Chemnitz. En outre, le plus grand réseau de micro-électronique d'Europe, Silicon Saxony e.V., a été créé en Saxe, regroupant 253 entreprises et organisations membres, pour un total de 35000 emplois.

\section{Graphique 1 : Taux de croissance de la part des emplois de R\&D dans un échantillon de régions}

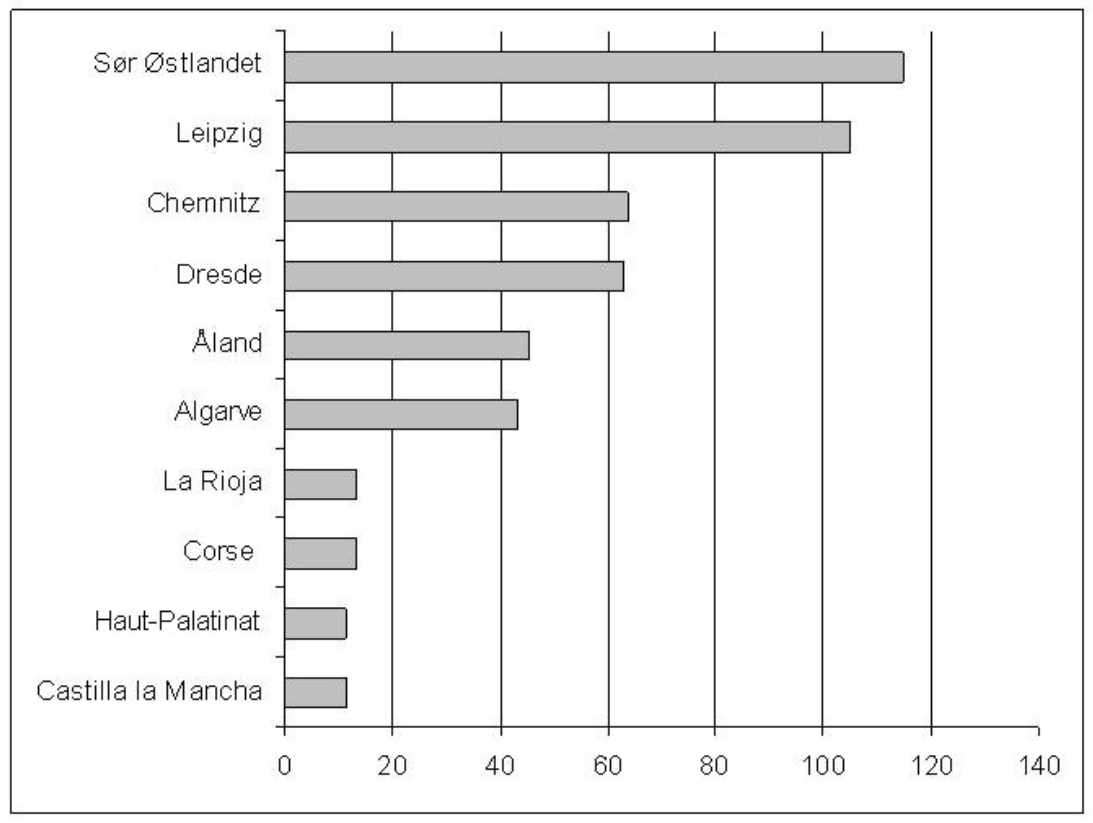

Source : Eurostat, Statistik kurz gefasst, Wissenschaft + Technologie, 6/2005. 
En Saxe, le soutien à la R\&D et à l'innovation émane du ministère saxon de l'Economie et du Travail (SMWA) et du ministère saxon de la Science et de l'Art (SMWK) ${ }^{9}$. L'essentiel des programmes publics de soutien est mis en œuvre et administré par la SAB.

La Saxe promeut la recherche et l'innovation dans le cadre du financement de projets coopératifs ou individuels de $R \& D$ et dans celui du programme d'aide à l'emploi hautement qualifié dans les PME (Innovationsassistentenprogramm) et de soutien au transfert de technologies, l'aide au regroupement étant l'action prédominante. Elle consiste à promouvoir des projets faisant coopérer plusieurs entreprises ou bien des entreprises et des organismes de recherche (universités et structures extra-universitaires). La collaboration d'entreprises et d'établissements d'enseignement supérieur peut être particulièrement féconde en matière d'innovation. En 2006, 60 projets individuels et 281 projets coopératifs ont été acceptés, soit des enveloppes totales de 18,9 millions $€$ et 86,7 millions $€$. Dans le cadre du programme «Soutien d'assistants à l'innovation », le gouvernement régional soutient la mise en place et la consolidation de capacités de R\&D dans des PME. Les diplômés et chercheurs aux qualifications particulièrement élevées, issus d'universités, de Fachhochschulen, d'écoles professionnelles ou d'organismes de recherche sont encouragés à élaborer au sein d'entreprises des projets au contenu innovant, axés sur la technologie. Au total, 19,5 millions $€$ ont été alloués à cette fin depuis 1995, dont 1,7 million en 2006. Dans le cadre du soutien au transfert de technologie, ce sont 103 millions $€$ qui ont été accordés depuis 1991, dont 6,6 millions en 2006. Les programmes de soutien de l'Etat libre de Saxe sont surtout financés à l'aide de fonds structurels européens. Ces fonds permettent au Land de financer à la fois la technologie dans le cadre de programmes de soutien à la $R \& D$ et des investissements dans des infrastructures de R\&D. Les moyens provenant des fonds structurels européens ont pour avantage de permettre une planification à long terme et, ainsi, la pérennisation de la politique de recherche et d'innovation en Saxe. Sont particulièrement soutenus les secteurs suivants: technique de production, nouveaux matériaux, micro- et nanotechnologies, aéronautique et aérospatiale, technologies des surfaces et revêtements, et biotechnologies.

En Saxe, la politique économique s'est concentrée ces dernières années sur l'attraction d'investissements étrangers dans le secteur de la haute technologie. D'une manière générale, le soutien aux investissements et à la recherche peut être utilisé en ce sens. Le financement de la recherche et de bonnes

\footnotetext{
${ }^{9}$ Le budget total de la Saxe représente 16,1 milliards $€$ pour 2008. 1,96 milliard $€$ est attribué au SMWA et 1,75 milliard au SMWK. Le SMWA a estimé à 471 millions $€$ l'enveloppe allouée au soutien économique général (dont 86 millions $€$ pour le soutien à la recherche et à la technologie) et 317 millions $€$ pour les priorités du Fonds européen de développement régional (FEDER).
} 
infrastructures de R\&D sont en effet d'importants facteurs d'implantation ${ }^{10}$. Le soutien public apporté à la micro- et à la nanoélectronique dans le Land de Saxe en constitue un exemple particulièrement intéressant. Depuis 1998, le BMBF soutient ce secteur à hauteur de 159 millions $€$ en Saxe. Grâce à ce financement, c'est dans cette région qu'a été réalisée la première chaîne de production au monde pour galettes de silicium de $300 \mathrm{~mm}$, tout à fait opérationnelle. Des moyens publics de financement ont permis de créer le réseau «Silicon Saxony », au sein duquel coopèrent de nombreux partenaires issus de grandes entreprises (Infineon, AMD par exemple), de PME et d'organismes publics de recherche (Centre Fraunhofer pour les technologies nanoélectroniques - CNT par exemple) innovants. Cette compétitivité accrue contribue à ce qu'un semiconducteur provenant d'Europe sur deux soit fabriqué en Allemagne. Les entreprises allemandes sont des leaders technologiques dans le domaine du développement de la lithographie pour produire des structures encore plus petites et performantes.

Pour la période 2007-2013, les fonds structurels européens alloués à la Saxe représentent 3,9 milliards $€ .78 \%$ de ces moyens proviennent du Fonds européen pour le développement régional (FEDER), et $22 \%$ du Fonds social européen (FSE).

Pour la période 2007-2013, la Saxe a défini les priorités suivantes ${ }^{11}$ :

- axe de priorité $\mathrm{n}^{\circ} 1:$ renforcement de l'innovation, de la science, de la recherche. 1079 millions $€$ (part de l'ensemble des subventions FEDER : $35 \%$ );

- axe de priorité $\mathrm{n}^{\circ} 2$ : amélioration des infrastructures de formation. 236 millions $€(7,6 \%)$;

- axe de priorité $\mathrm{n}^{\circ} 3$ : accroissement de la compétitivité des entreprises industrielles. 587 millions $€(19 \%)$;

- axe de priorité $\mathrm{n}^{\circ} 4$ : amélioration des infrastructures de transports. 573 millions $€(18,6 \%)$;

- axe de priorité $\mathrm{n}^{\circ} 5$ : développement et amélioration des infrastructures favorisant une croissance économique durable. 571 millions $€(18,5 \%)$.

Pour la période de financement en cours, la Saxe se concentre donc sur le renforcement de l'innovation, de la science et de la recherche dans le cadre de la priorité $n^{\circ} 1.35 \%$ de l'ensemble des subventions FEDER sont consacrés à cette priorité.

Par le passé, les subventions provenant des fonds structurels européens ont essentiellement contribué au financement de la politique saxonne de recherche

\footnotetext{
${ }^{10}$ Comme il a été dit plus haut, il y a en Saxe quelques organismes de recherche extra-universitaires surtout financés par l'Etat fédéral. Cela signifie que les moyens attribués par le Bund ont apporté une contribution essentielle à la consolidation du pôle de recherche saxon.

${ }_{11}$ Programme opérationnel de l'Etat libre de Saxe pour le Fonds européen de développement régional (FEDER) dans un objectif de convergence pour la période de financement 2007-2013. Ministère saxon de l'Economie et du Travail, Dresde.
} 
et d'innovation. Une grande partie des mesures et des programmes régionaux est indirectement financée par des fonds structurels. Pour la période 2007-2013, ces mêmes moyens ont surtout été consacrés au financement d'infrastructures de recherche et à l'innovation dans les régions européennes, ce qui favorise la mise en œuvre de la politique saxonne de recherche et d'innovation.

\section{Mécanismes de coordination de la politique d'innovation}

La structure fédérale de l'Allemagne entraîne souvent un chevauchement des compétences entre échelon national et régional, notamment en ce qui concerne la recherche extra-universitaire. Dans le domaine de la politique de l'enseignement supérieur, qui relève en fait des Länder, des subventions et des initiatives émanant de l'Etat fédéral, en particulier l'Initiative d'Excellence, ont imposé une plus forte coordination. S'ajoutent à cela des programmes de financement du Bund ainsi que les fonds structurels européens, qui requièrent une coordination et une harmonisation entre échelon national et Länder. Cette partie est donc consacrée aux mécanismes de coordination de la politique d'innovation en Allemagne.

De nombreux mécanismes de pilotage permettent de coordonner et d'harmoniser les financements émanant des ministères fédéraux (BMBF et BMWi) et des Länder :

- coordination informelle entre ministères des différents niveaux décisionnels et porteurs de projets ;

- échange de données régulier entre les différentes entités, afin d'éviter les doublons ;

- groupes de travail formels assurant régulièrement l'harmonisation entre l'Etat fédéral et les Länder (par exemple dans le cadre de la mission commune pour l'«Amélioration de la structure économique régionale $\gg)$;

- groupes de travail informels communs dans le cas de projets particuliers de soutien à la R\&D (s'agissant par exemple de grands projets d'investissement comme les 3 milliards $€$ investis par AMD à Dresde ou les 3 milliards investis par Infineon). Ces groupes de travail réunissent des représentants du $\mathrm{BMBF}$, de la $\mathrm{KfW}$, de la $\mathrm{SAB}$, du SMWA, du SMWK, des universités et des organismes de recherche extra-universitaires.

La réforme du fédéralisme a réduit les compétences de l'Etat fédéral en matière d'enseignement supérieur. Début 2008 a été créée la Conférence scientifique commune (GWK) destinée à coordonner la politique de la science et de la recherche. Ce mécanisme de coordination formel se substitue à la commission Bund-Länder (BLK) pour la planification de l'éducation et le soutien à la recherche. Sont membres de la GWK les ministres fédéraux et régionaux en 
charge de la science, de la recherche et des finances. Cette conférence coordonne toutes les questions de soutien à la recherche touchant à la fois l'Etat fédéral et les Länder; elle assure notamment :

- la coordination de la politique nationale, européenne et internationale de la science et de la recherche dans le but d'accroître la compétitivité du pôle scientifique et de recherche allemand à l'échelle internationale ;

- des interventions dans des cas d'importance suprarégionale, s'agissant $\mathrm{du}$ financement d'organismes et de projets de recherche scientifique en dehors d'établissements d'enseignement supérieur; dans le cas de projets scientifiques et de recherche dans des établissements d'enseignement supérieur et dans celui de la construction de laboratoires de recherche dans des universités impliquant des équipements lourds ;

- la communication réciproque d'informations également sur des projets et décisions cruciaux qui ne font pas l'objet d'un financement commun.

La Conférence scientifique commune a donc une position de premier plan en matière de coordination de la politique de recherche pour les questions concernant à la fois l'Etat fédéral et les Länder. Cela vaut en particulier pour la coordination de la recherche extra-universitaire.

Comme cela a déjà été montré, les financements émanant des fonds structurels européens sont particulièrement importants. La coordination et l'harmonisation de la planification de projet dans le cadre des fonds structurels européens 2007-2013 ont obéi à la logique fédérale du pays. Les négociations sur l'attribution de subventions se sont déroulées à différents échelons. Les organismes régionaux compétents ont défini les grandes lignes du financement à venir en s'appuyant sur un diagnostic des forces et des faiblesses propres à leur région, sur le bilan du soutien apporté jusque-là et sur les nouvelles contraintes résultant des règles d'attribution des fonds structurels. Toujours à l'échelon régional, il a été procédé à des analyses des forces et des faiblesses locales. Il a également été fait appel aux résultats de l'évaluation intermédiaire de la période de financement 2000-2006. La plupart des Länder ont organisé des ateliers et des tables rondes rassemblant parfois plusieurs centaines de participants. Les résultats de ces consultations et discussions régionales ont fait l'objet d'échanges lors des rencontres communes des gouvernements des Länder et de l'Etat fédéral. En Saxe, la participation des partenaires à l'élaboration de programmes a commencé en avril 2005 par une réunion de lancement. Les partenaires à l'échelon régional ont alors été informés des conditions cadre de la nouvelle période de financement, et les participants ont été invités à présenter leurs idées et des indications sur l'orientation de la période à venir. Des représentants de différents acteurs comme les syndicats, les associations patronales et des organisations non gouvernementales ont participé aux consultations et aux réunions d'information. En Saxe, des conférences 
régionales regroupant 70 à 80 participants ont été organisées dans les circonscriptions de Chemnitz, Dresde et Leipzig. On peut donc qualifier ce processus de coordination d'ascendant (bottom-up).

Il ressort des entretiens avec des représentants du soutien à l'innovation en Saxe que les mécanismes informels de coordination jouent un rôle absolument essentiel. Les personnes interrogées ont indiqué que les collaborateurs des ministères régionaux compétents (SMWA et SMWK) et ceux de la SAB ont des visions communes en matière de $R \& D$ et que les ministères saxons travaillent en étroite collaboration avec cette banque, principale structure de financement du Land, qui met en œuvre et administre les programmes d'aides. Par ailleurs, les ministères fédéraux concernés sont souvent en contact. De nombreux mécanismes informels de coordination existent par conséquent, permettant d'assurer une meilleure cohérence des politiques d'innovation des différents niveaux décisionnels ${ }^{12}$.

En Allemagne, la POlitique D'INnOvation se pratique à quatre échelons différents. Au niveau des collectivités territoriales, l'accent est mis sur la politique de promotion territoriale, et en particulier sur l'aide à la création d'activité et à l'implantation d'entreprises. L'exemple de la Saxe a permis de montrer que de nombreuses possibilités de soutien existent au niveau régional. L'enseignement supérieur et le financement des universités sont en outre d'abord du ressort des Länder. L'Etat fédéral mène une politique de recherche et d'innovation assurée par le ministère de l'Education et de la Recherche et par celui de l'Economie. Un grand nombre de mesures de financement soutiennent la recherche, le développement et les processus d'innovation. Le Bund soutient en outre les réseaux régionaux à travers de nombreuses actions, favorisant les régions au moyen de concours. Cette remarque vaut également pour le soutien à la recherche d'excellence dans les établissements d'enseignement supérieur, auxquels l'Initiative d'Excellence de l'Etat fédéral permet d'octroyer des moyens en plus des financements des Länder. Le soutien apporté par l'Union européenne dans le cadre des fonds structurels européens est particulièrement important dans les Länder de l'Est.

\footnotetext{
12 Outre les mécanismes formels de coordination, il existe depuis 1957 le Conseil scientifique, une entité consultative importante en matière de politique scientifique. Cette instance consultative est portée conjointement par les gouvernements des Länder et par l'Etat fédéral, et cofinancée pour moitié par chacun ; sa mission consiste à élaborer des recommandations générales sur l'évolution thématique et structurelle de la science, de la recherche et de l'enseignement supérieur. Le Conseil scientifique donne des recommandations dans deux domaines de la politique scientifique : il s'agit d'une part des questions relatives aux structures, à la performance, au financement et à l'évolution d'organisations scientifiques, en particulier des universités, des Fachhochschulen et des organismes de recherche extra-universitaires; et il doit d'autre part émettre des recommandations sur des questions générales touchant le système de recherche allemand, sur des aspects structurels ainsi que sur la planification, l'évaluation et le pilotage de certains domaines et de certaines spécialités. Le Conseil scientifique réunit des chercheurs, des représentants des organisations scientifiques, du Bund, des Länder ainsi que des représentants de la société civile.
} 
De nombreux mécanismes permettent d'assurer l'harmonisation et la coordination de la politique d'innovation entre les différents niveaux décisionnels. Les mécanismes informels, dus aux contacts entre personnes, jouent un rôle tout aussi important que les mécanismes formels et les commissions de coordination.

Cette contribution a montré qu'une vision systémique s'est imposée à tous les échelons de la politique d'innovation. Au fil de cette politique systémique, l'aide au regroupement a encouragé la création de réseaux, souvent composés d'organismes publics de recherche et d'entreprises privées. Au travers de programmes cadre de recherche, l'Union européenne promeut des consortiums internationaux, les ministères fédéraux soutiennent des réseaux thématiques et liés à des régions (BioRegio, InnoRegio par exemple); enfin, les Länder, la Saxe en tout cas, encouragent la recherche coopérative. La littérature consacrée à l'économie d'innovation interprète la coopération des acteurs d'un système d'innovation comme un facteur de succès essentiel ${ }^{13}$. Les interactions et les interdépendances entre les acteurs sont l'objet central de l'analyse des processus d'innovation. «Les entreprises n'innovent pratiquement jamais dans l'isolement» (Edquist, 1997, p. 20). Dans ce domaine, on considère que la coopération et la mise en réseau des entreprises ont une influence positive sur l'innovation. Ces dernières années, on s'est davantage intéressé au lien entre universités et institutions publiques de recherche d'une part et activités d'innovation des entreprises d'autre part. Les données empiriques montrent que la recherche issue des universités donne des impulsions importantes pour les activités d'innovation des entreprises ${ }^{14}$. Dans une perspective de politique d'innovation, les universités et les organismes de recherche extra-universitaires sont donc considérés comme un élément crucial d'un système d'innovation. Un grand nombre d'instruments de soutien encouragent l'intégration d'institutions de recherche à des projets menés conjointement avec des entreprises.

Traduction de Marie GRAVEY

\section{Indications bibliographiques}

BECKER P., ZAUn N., Die neue strategische Planung der europäischen Kohäsionspolitik in Theorie und Praxis, Stiftung Wissenschaft und Politik, Deutsches Institut für Internationale Politik und Sicherheit, Berlin, 2007

BUNDESFINANZMINISTERIUM, Aktualisierung der Halbzeitbewertung des Gemeinschaftlichen Förderkonzepts 2000-2006 (GFK) für den Einsatz der Strukturfonds in den neuen Bundesländern und im Ostteil Berlins, Bundesfinanzministerium, Berlin, 2005

BUNDESMINISTERIUM FÜR WIRTSCHAFT UND TECHNOLOGIE (BMWI), Nationaler Strategischer Rahmenplan (NSRP) für den Einsatz der EU-Strukturfonds in der Bundesrepublik Deutschland 2007-2013, BMWi, Berlin, 2007

\footnotetext{
${ }^{13}$ Pour une présentation des systèmes d'innovation, voir Lundvall (1992), Nelson (1993) et Edquist (1997).

${ }^{14}$ Voir notamment Jaffe (1989), Mansfield (1995; 1998), Mowery und Sampat (2005).
} 
EDQUIST C., «Systems of Innovation Approaches - Their Emergence and Characteristics», in EDQUIST C. (ed), Systems of Innovation - Technologies, Institutions and Organizations, Pinter, Londres, 1997, p. [n.c.]

COMMISSION EUROPÉENNE, Wachsende Regionen, wachsendes Europa - Vierter Bericht über den wirtschaftlichen und sozialen Zusammenhalt, Bruxelles, Commission européenne, 2007a

COMMISSION EUROPÉENNE, Grünbuch - Der Europäische Forschungsraum: Neue Perspektiven, Bruxelles, Commission européenne, $2007 \mathrm{~b}$

FIER A., HARHOFF D., «Die Evolution der bundesdeutschen Forschungs- und Technologiepolitik: Rückblick und Bestandsaufnahme», Perspektiven der Wirtschaftspolitik, vol. 3, n 3, août 2002, p. 279-301

JAFFE A. B., «Real Effects of Academic Research», American Economic Review, vol. 79, n 5, décembre 1989 , p. $957-970$

Lundvall B.-Å., Borrás S., «Science Technology, and Innovation Policy», in FAGERBERG J., Mowery D. C., Nelson R. R. (ed), The Oxford Handbook of Innovation, Oxford University Press, Oxford, 2005, p. 359631

LundVALL B.-Å., National Systems of Innovation: Towards a Theory of Innovation and Interactive Learning, Pinter Publishers, Londres, 1992

MANSFIELD E., «Academic Research Underlying Industrial Innovations: Sources, Characteristics, and Financing», Review of Economics and Statistics, vol. 77, $\mathrm{n}^{\circ}$ 1, février 1995, p. 55-65

MANSFIELD E., «Academic Research and Industrial Innovation: An Update of Empirical Findings», Research Policy, vol. 26, $\mathrm{n}^{\circ} 7-8$, avril 1998, p. 773-776

Mowery D. C., SAMPat B. N., «Universities in National Innovation Systems», in FAGERBERG, J., MOWERY D. C., Nelson R. R. (ed), The Oxford Handbook of Innovation, Oxford University Press, Oxford, 2005 , p. 209-239

Nelson R. R., National Innovation Systems: A Comparative Analysis, Oxford University Press, Oxford, 1993

OCDE, Changement technique et politique économique, OCDE, Paris, 1980

SÄCHSISCHES STAATSMINISTERIUM FÜR WIRTSCHAFT UND ARBEIT, Operationelles Programm des Freistaates Sachsen für den Europäischen Fonds für regionale Entwicklung (EFRE) im Ziel „Konvergenz" in der Förderperiode 2007 bis 2013, Sächsisches Staatsministerium für Wirtschaft und Arbeit, Dresde, 2007

SEllenthin M. O., «Is There a Local Innovation Policy to Support Academic Start-up Activities? An Exploratory Case Study of Heidelberg», Brussels Economic Review, Vol. 49, n 4, hiver 2006, p. 293-310. 



\title{
Enjeux et perspectives de la politique des pôles de compétitivité en France
}

\author{
Bernard FROMENT
}

En 2004, le gouvernement français a lancé une politique de pôles de compétitivité, visant notamment à améliorer les transferts de technologies entre science et industrie dans le contexte de la Stratégie de Lisbonne. Les 71 réseaux aujourd'hui labellisés, répartis selon trois catégories (pôles mondiaux, à vocation internationale et nationaux), sont financés par le Fonds Unique Interministériel (FUI), et, par le biais d'appels à projets, par l'Agence Nationale de la Recherche (ANR). La contribution de Bernard Froment, responsable jusqu'en 2009 du bureau de la valorisation, de la propriété intellectuelle et du partenariat du ministère de l'Enseignement supérieur et de la Recherche, permet de retracer le processus ainsi que les objectifs de la stratégie interministérielle des pôles de compétitivité, associée par ailleurs à une politique plus spécifique de promotion de la recherche.

Pour revenir dans un premier temps sur le contexte des pôles de compétitivité, je rejoins la remarque de Sonja R. Kind sur la différence entre «réseau » et «cluster ». Effectivement, je partage cette analyse entre des réseaux qui sont issus d'une construction organisée et des clusters qui sont plutôt naturellement issus de la coopération géographique locale ; toutefois, la sollicitation locale et l'incitation permettent de construire des réseaux locaux que l'on pourra qualifier de clusters. La particularité forte des clusters des pôles de compétitivité en France (ce ne sont bien sûr pas les seuls clusters fondés sur ce modèle au niveau mondial) est la construction du pôle de compétitivité sur trois pieds, cette obligation étant inscrite au cahier des charges : un pied «entreprise », un pied « recherche » et un pied « formation».

\section{Labellisation et stratégie des pôles de compétitivité}

L'appel à projets "pôles de compétitivité » date de 2004. 105 projets ont été soumis en 2004 et 67 pôles ont été labellisés initialement, devenus 66 puisqu'il y a eu fusion de deux pôles, puis 71 plus récemment avec la nouvelle labellisation de cinq autres pôles. Avant d'entrer dans la vision du ministère de l'Enseignement supérieur et de la Recherche, je souhaite remarquer la difficulté - classique - entre prendre l'arrosoir pour arroser très large et concentrer les efforts sur quelques pôles. Ainsi, au moment de la labellisation en 2005, il y a eu une hésitation assez forte entre la labellisation d'une quinzaine de pôles et d'une soixantaine de pôles ; c'est de cette hésitation forte qu'est née la labellisation d'une soixantaine de pôles, mais avec l'identification au sein de ces pôles de trois catégories, avec des pôles dits « mondiaux », des pôles dits « à vocation 
mondiale » et des pôles dits " nationaux ». Je ne suis pas sûr que les appellations aient été heureuses et saluées par tous mais il s'agit là des appellations consacrées. Cette difficulté du choix entre la concentration des efforts ou le travail sur un faisceau plus large s'est présentée : elle naît de la divergence entre soutien de l'excellence et aménagement du territoire. De cette soixantaine de pôles, une large expérience est née. Une évaluation a été conduite dont les résultats sont pour partie publics et consultables sur Internet. Au travers de cette évaluation, le concept de "pôle » est validé, mais une évolution vers un ancrage plus fort entre la politique de pôles et les autres politiques conduites est souhaitée. En effet, il faut rappeler qu'au niveau français, les pôles de compétitivité relèvent d'une politique interministérielle avec la participation de ministères transversaux, avec des ministères comme celui en charge de l'aménagement du territoire, celui en charge des entreprises, celui en charge de l'Enseignement supérieur et de la Recherche mais aussi des ministères thématiques, notamment l'agriculture, la défense, la santé. Nous sommes donc sur une politique interministérielle et comme chacun sait, dès qu'on est nombreux autour d'une table et qu'on doit construire quelque chose ensemble, chacun doit faire des concessions et dans le même temps adhérer au résultat final. Toute la difficulté est là.

\section{L'« exception recherche » en matière de financement...}

Au sein de cette stratégie d'ensemble des pôles de compétitivité, le ministère de l'Enseignement supérieur et de la Recherche a une position légèrement différente de l'ensemble des autres ministères du fait de l'« exception recherche». Nous ne pouvons pas, en tant que ministère de l'Enseignement supérieur et de la Recherche, surtout sur le volet recherche, accepter de financer des projets de recherche qui ne soient pas sélectionnés prioritairement sur la qualité scientifique. Donc, nous avons choisi de ne pas contribuer au financement interministériel commun des pôles de compétitivité. Nous sommes bien sûr impliqués pour le pilotage des pôles de compétitivité, mais en termes de financement, nous avons confié l'essentiel de notre effort de financement de la recherche à l'Agence Nationale de la Recherche (ANR), qui a une expertise sur le plan de la qualité scientifique des projets. Ses critères sont d'abord centrés sur l'excellence scientifique, ce qui n'est pas compatible avec la prise en compte d'autres critères d'attribution tels que le développement économique, l'aménagement du territoire.... Nous sommes donc dans la stratégie des pôles de compétitivité, mais avec un positionnement différent en termes de financement. En conclusion, l'ensemble des ministères a construit en commun un fonds d'abondement, dit «Fonds Unique Interministériel» (FUI) qui abonde le financement des projets issus des pôles de compétitivité et, en parallèle, il y a une autre source de financement sur le plan de la recherche par le biais des appels à projets de l'Agence Nationale de la Recherche. Sur le plan des mon- 
tants financiers, il est important d'observer que sur environ 800 millions $€$ attribués annuellement par l'Agence Nationale de la Recherche, environ 200 millions $€$ sont attribués à des projets de recherche issus de pôles de compétitivité, qui ont été labellisés par ces pôles. Cela ne signifie pas que 200 millions $€$ sont allés vers les entreprises; il s'agit bien de 200 millions $€$ affectés à des projets de recherche issus des pôles de compétitivité. Au sein des 200 millions $€$, il y a une répartition entre recherche publique et entreprises en fonction de la construction de chacun des projets. C'est une particularité sur le plan $\mathrm{du}$ fonctionnement en termes d'abondement financier des pôles de compétitivité.

Mais, ce qui paraît important au ministère de l'Enseignement supérieur et de la Recherche, c'est l'effet " cluster » avant de parler du financement. Certes le financement est important, mais dans certains types de réseaux, il n'y a pas de financement et pourtant les gens vont vers ce type de réseaux. Cela montre qu'il y a une autre vie que celle du ticket financier dans la politique de réseaux ou de clusters. Ce qui intéresse donc le ministère de l'Enseignement supérieur et de la Recherche, c'est la dynamique qui est introduite par l'effet «cluster », le développement et l'amplification de cette dynamique.

\section{...et la politique de pôles du ministère de l'Enseignement supérieur et de la Recherche}

En parallèle avec la mise en place des pôles de compétitivité, le ministère de l'Enseignement supérieur et de la Recherche a conduit une politique de pôles qui se retrouve notamment au travers des pôles de recherche et d'enseignement supérieur (PRES), avec comme vocation de structurer géographiquement l'effort de recherche et d'enseignement supérieur. La structuration géographique montre, en général, un parallélisme avec les pôles de compétitivité qui sont euxmêmes essentiellement au niveau d'une région, même si certains sont multirégionaux. Les pôles de recherche et d'enseignement supérieur sont un outil allant dans ce sens, les réseaux thématiques et de recherche avancée (RTRA) sont aussi des outils de structuration, de mise en réseau qui, pour l'essentiel, sont aussi autour de la proximité, un seul des 13 réseaux thématiques de recherche avancée étant géographiquement réparti sur le territoire.

\section{Stimuler les transferts de technologies}

La stratégie du ministère de l'Enseignement supérieur et de la Recherche se traduit aussi par un ensemble de dispositifs dans la même dynamique que les pôles de recherche et d'enseignement supérieur, autour de la valorisation de la recherche et du transfert de technologies. Une initiative, lancée en 2005 avec la coopération financière de l'ANR, a consisté à créer des dispositifs mutualisés en région pour le développement du transfert de technologies depuis la recherche 
publique vers l'économie. Ces dispositifs mutualisés sont inscrits dans l'axe « structuration régionale » et sont très complémentaires des pôles de compétitivité. Caricaturalement, on pourrait situer le pôle de compétitivité, dans la relation entre recherche publique et entreprise, comme une structure qui va travailler sur le mode «market pull», c'est-à-dire que la relation entre l'entreprise et la recherche publique va permettre de «tirer»l'activité de recherche pour alimenter les besoins qui apparaissent au niveau de l'entreprise. Pour les dispositifs de valorisation et de transferts de technologies, le positionnement se situerait plutôt dans un mode «technology push », c'est-à-dire que la recherche publique, soit par des recherches propres, soit par des recherches conduites avec des partenaires, a abouti à des produits transférables, des résultats de recherche qui font l'objet de brevets ou autres protections. L'objectif de la recherche publique étant de mettre à disposition de la société les résultats de la recherche publique qu'elle a financée, ces résultats ont pour vocation naturelle d'être transférés vers le monde socioéconomique. Ces dispositifs mutualisés ont pour vocation d'organiser ce transfert. Leur efficacité et le professionnalisme du traitement sont essentiels, car une technologie a parfois été développée pour une application dans un domaine particulier, mais une phase de marketing technologique montre parfois que cette même technologie peut être utilisable dans bien d'autres domaines que celui pour lequel elle a été développée.

Il existe donc une très forte conjonction de travail entre cette fonction de transfert de technologies et les pôles de compétitivité. Ces initiatives rejoignent souvent la structuration en pôles de recherche et d'enseignement supérieur, voire en « campus ». La stratégie du ministère de l'Enseignement supérieur et de la Recherche s'oriente par conséquent vers la montée en puissance d'un certain nombre de « services de site » en matière de valorisation de la recherche et de transfert de technologies, correspondant en général au niveau régional (mais parfois au niveau infra ou supra, suivant le facteur d'échelle des régions). Lorsque le pôle de recherche et d'enseignement supérieur existe, ce service de site en serait l'opérateur de valorisation. Si ces services de site devenaient de vrais outils de coopération qui représentent l'ensemble de la recherche publique au sein de pôles de compétitivité, le système aurait alors probablement beaucoup gagné en efficience.

\section{La formation au cœur des pôles de compétitivité}

Mon propos est essentiellement tourné vers la structuration et très peu vers le financement; il ne s'agit pas de provocation, mais seulement d'un constat simple: le financement n'est que le résultat d'une bonne coopération entre recherche publique et entreprises. $\mathrm{Si}$, en effet, il existe une bonne coopération, il émergera naturellement de bons projets, et ces bons projets seront financés soit dans le cadre interministériel par le FUI, soit dans le cadre de projets de recherche par l'ANR. 
Un des points qui, aujourd'hui, est encore en difficulté dans les pôles de compétitivité, c'est le pied «formation ». Je rappelais dans l'introduction qu'un pôle de compétitivité, c'est un système à trois pieds : un pied «entreprise », un pied « recherche » et un pied «formation». Le pied « entreprise» est évident dans la notion de cluster puisque c'est là que se produisent l'innovation et la production. Le pied « recherche », apparaît rapidement, parce que produire plus et de manière plus innovante nécessite assez directement de la recherche. Mais si on oublie le pied «formation», nos pôles de compétitivité auront une durée de vie efficiente limitée et, après-demain, risquent d'être en difficulté. Il est donc capital et urgent de développer ce pied «formation », qu'il s'agisse de formation initiale ou de formation continue, si nous voulons que les pôles de compétitivité soient vraiment à l'origine de l'économie efficiente et innovante d'après-demain. C'est pour le ministère de l'Enseignement supérieur et de la Recherche un des points importants, et nous pensons aujourd'hui qu'aucun des acteurs ne s'est suffisamment mobilisé sur cet aspect. Il s'agit d'un défaut de jeunesse des pôles qui peut encore être corrigé si les partenaires se mobilisent sur cet aspect, la structuration des pôles de compétitivité étant tout de même récente.

\section{Favoriser l'entente entre les acteurs des pôles}

Si vous permettez que je parle un peu d'expérience personnelle, au moment de l'appel à projets "pôles de compétitivité », je n'étais pas en poste au ministère, j'étais en poste dans mon université, en région. Ma carrière, sur le plan recherche, s'est effectuée au sein d'un laboratoire très tourné vers les entreprises, preuve en est sa labellisation Carnot lors du premier appel à projets. A cette période, j'occupais des fonctions de direction de formation continue dans l'université. Là encore, $\mathrm{j}$ 'étais au contact régulier des entreprises sur le plan de la formation. Sur le terrain, en tout cas dans la région dans laquelle j'étais, j'ai pourtant trouvé en 2004 que cet appel à projets avait réellement introduit une dynamique que nous n'avions jamais vue sur la relation entre l'ensemble des partenaires. Les partenaires universitaires et les entreprises se réunissaient auparavant pour parler parfois de formation ou de recherche et souvent d'insertion des jeunes diplômés. Les partenaires "pouvoirs publics » avaient l'habitude de se rencontrer dans certains cas avec les entreprises, dans d'autres cas avec la recherche ou l'enseignement supérieur. Pour la préparation de la réponse à l'appel à projets "pôles de compétitivité ", c'était la première fois qu'ils se retrouvaient tous autour d'une même table pour parler, surtout pour s'écouter... et même aller jusqu'à s'entendre. C'était la première fois que l'on avait cette dynamique globale sur l'ensemble des partenaires autour d'une même table. Le pôle de compétitivité dans lequel j'étais impliqué a finalement été labellisé. Ce que j'ai retenu de cette expérience au niveau de la gouvernance de l'université à laquelle je participais, c'est que même si le pôle n'avait pas été 
labellisé, nous avions fait un grand pas parce que nous avions enfin réussi à nous écouter tous ensemble, ce que nous n'avions jamais réussi à faire auparavant.

Pour reboucler sur la notion de "réseau » et de "cluster 》, cet exemple illustre bien le fait que si les réseaux s'organisent, les clusters ne s'organisent pas, mais que l'incitation à la création de réseaux fait parfois vraiment naître des clusters ; tout simplement parce que dans certains endroits, il n'y avait pas eu d'initiative antérieure. Il est vrai que ce n'est pas forcément le cas de toutes les régions. Par exemple sur le site de Grenoble, il est très clair que l'entente entre recherche, formation, entreprises et collectivités est très ancienne, mais il existe trop peu de cas à citer, et la dynamique des pôles de compétitivité est capitale pour les favoriser. C'est grâce à cette entente que l'on peut espérer être demain correctement positionnés dans l'économie de la connaissance.

\section{Financement et réseaux sont dissociés: l'exemple du crédit d'impôt recherche}

Pour conclure sur l'aspect financement et sa désolidarisation de la notion de réseaux, j'illustrerai par le développement du crédit d'impôt recherche. Le crédit d'impôt recherche est un outil de financement de la recherche en entreprise qui n'est lié ni à une thématique, ni à une appartenance à un réseau, ni à un mode de fonctionnement particulier de la recherche en entreprise (qu'elle soit interne à l'entreprise, sous-traitée, co-traitée, tous les modes de fonctionnement sont valides). La montée en puissance du dispositif " crédit d'impôt recherche » actuel est bien là pour montrer que la stratégie du ministère de l'Enseignement supérieur et de la Recherche sépare bien l'incitation au réseau ou au cluster par toutes les actions menées, du financement. Le financement de la recherche se fait sur des critères scientifiques d'excellence (financement par l'Agence Nationale de la Recherche) ou des critères d'intérêt pour le développement économique. Dans ce dernier cas, nous estimons que ce n'est pas au ministère de décider si cette recherche est pertinente ou non, c'est à l'entreprise, qui prend alors ses responsabilités... et nous l'aidons par le biais du crédit d'impôt recherche; une incitation au partenariat avec la recherche publique existe tout de même dans le mode de calcul dudit crédit d'impôt.

Ainsi, la part de financement dans un système de réseaux ou de clusters a pour seul objectif d'y attirer les partenaires, les bénéfices réels seront dans l'efficience de cette logique de clusters. 


\section{Stratégies des acteurs publics français et dynamiques locales : les dilemmes du jacobinisme}

\section{Pierre PAPON}

Dans le contexte d'une concurrence accrue entre les territoires à l'échelle globale, il apparaît désormais nécessaire de mener des actions concertées impliquant l'ensemble des acteurs du système de recherche et d'innovation français (Etat, collectivités territoriales, entreprises, universités et centres de recherche extra-universitaires). Dans la contribution qui suit, Pierre Papon, professeur émérite à l'Ecole Supérieure de Physique et de Chimie Industrielles (ESPCI) et président du groupe de travail FutuRIS sur l'internationalisation des activités de R\&D et d'innovation et son impact sur les territoires au sein de l'Association Nationale de la Recherche et de la Technologie (ANRT), dresse un état des lieux de la recherche en France pour exposer ensuite le diagnostic et les recommandations adressés par FutuRIS à l'Etat et aux régions, en vue de promouvoir la compétitivité territoriale aux niveaux national et international.

Nous allons nous intéresser essentiellement aux problèmes de la stratégie des acteurs publics français en matière de recherche et d'innovation en relation avec les dynamiques locales. Pour mieux souligner la complexité de la relation Etatrégions dans ce domaine, nous avons donné un sous-titre à cette contribution au débat: «Les dilemmes du jacobinisme». Il veut montrer combien la France se trouve prise dans ce domaine, comme dans quelques autres d'ailleurs, entre le marteau de l'Etat central et l'enclume du pouvoir régional. Nous aborderons essentiellement trois points : nous commencerons par un rappel rapide du contexte français, puis nous rappellerons les évolutions du système de recherche et d'innovation confronté à la concurrence internationale et aux exigences de la " compétitivité ». Enfin, nous expliciterons les dilemmes du système français face au rapport politique nationale/politique régionale dans le domaine de la recherche et de l'innovation. Alors que l'Allemagne est, semble-t-il, confrontée à l'évolution du fédéralisme (en particulier pour la recherche et l'enseignement supérieur), la France, s'interroge encore, quant à elle, sur le rôle et la place des politiques régionales.

\section{Le contexte français}

Rappelons d'abord que la France consacre, aujourd'hui, 2,15\% de son PIB à la recherche et au développement, c'est, notons le, un pourcentage identique à celui de 1985. La dépense nationale de R\&D est passée par un pic à $2,33 \%$ du PIB en 1990, puis elle est retombée depuis lors à son niveau actuel. On doit remarquer aussi que $46 \%$ de la dépense intérieure de R\&D est financée sur fonds pu- 
blics (à 90-95\% par l'Etat). On remarque qu'il subsiste une petite inconnue statistique sur l'origine du financement public de la R\&D, qui n'est d'ailleurs pas si petite que cela, car le financement de la part des collectivités territoriales (régions, départements, municipalités) qui devient de plus en plus important en France est mal mesuré. On constate, par ailleurs, que si 54\% de la dépense intérieure de R\&D est financée sur fonds privés, $65 \%$ de cette dépense de R\&D est effectuée dans le secteur privé. Les évolutions récentes des dépenses nationales de R\&D semblent indiquer, toutefois, que les parts respectives des financements public et privé s'équilibreraient $(50 / 50)$. La part relativement importante de la recherche exécutée dans le secteur privé signifie donc qu'il existe des transferts de financements du public vers le secteur des entreprises, via des contrats de recherche des ministères vers les entreprises, en particulier dans le domaine de la défense qui est très important en France par son financement et ses activités (en Allemagne aussi d'ailleurs, mais certainement dans une moindre proportion).

Nous rappellerons aussi que le contexte institutionnel français est complexe avec, notamment, des universités et des écoles d'ingénieurs distinctes, nous dirons même une poussière d'écoles d'ingénieurs sous-critiques en recherche, mais aussi avec des organismes de recherche à vocation scientifique dont le rôle est important qui sont des Etablissements Publics à caractère Scientifique et Technique (EPST) dont les missions ont été redéfinies par la loi sur la recherche de 1982 : le Centre National de la Recherche Scientifique (CNRS), l'Institut National pour la Santé Et la Recherche Médicale (INSERM), l'Institut National de la Recherche Agronomique (INRA), etc. Une deuxième catégorie d'Etablissements Publics dits à caractère Industriel et Commercial (EPIC) ont des missions plus finalisées : comme le Commissariat à l'Energie Atomique (CEA) pour le nucléaire, le Centre National d'Etudes Spatiales (CNES) pour l'espace, l'Institut Français de Recherche pour l'Exploitation de la Mer (IFREMER) pour toutes les questions maritimes et des centres de recherche qui relèvent du ministère de la Défense. A ces organismes de recherche s'ajoutent des agences de financement: l'Agence Nationale pour la Recherche (l'ANR), OSEO-Innovation qui soutient les PME, l'Agence De l'Environnement et de la Maîtrise de l'Energie (l'ADEME) pour l'essentiel.

La gouvernance de ce système, on le conçoit, est loin d'être limpide, avec au centre du dispositif un ministère de la Recherche et de l'Enseignement supérieur (qui a souvent changé de nom au gré des changements de gouvernements) qui fixe les priorités, fait des arbitrages, mais aussi avec une tradition française de forte intervention du ministère des Finances s'agissant d'arbitrages. Il existe des interactions variables entre les organismes de recherche et les universités, l'interaction entre les universités et le CNRS est relativement forte avec un système de laboratoires mixtes cogérés par le CNRS et les universités et les écoles d'ingénieurs. De ce point de vue, ce système est assez différent du système allemand puisque les instituts Max-Planck sont très largement indépendants des universités, même s'ils sont dirigés, le plus souvent, par des 
universitaires. En France, les régions, et de façon générale les collectivités territoriales (régions, départements, municipalités), n'ont pas de responsabilité pour soutenir la recherche et l'innovation. Les deux lois de 1982 - la loi sur la recherche (ou loi Chevènement du nom du ministre qui l'a portée sur les fonts baptismaux) et la loi sur la décentralisation ou loi Defferre (du nom du ministre de l'Intérieur de l'époque, le maire de Marseille, qui en fut l'artisan) qui est probablement l'une des lois les plus importantes des vingt-cinq dernières années - n'ont transféré aucune responsabilité en matière de recherche et d'innovation aux régions (la loi Chevènement prévoyait seulement une concertation entre l'Etat et les régions pour l'élaboration de la politique nationale qui n'a, de fait, jamais été mise en œuvre). C'est une différence importante par rapport à la situation de la République fédérale ou des Länder jouent un rôle très important dans le financement de la recherche, même si, en France, les régions ont un rôle croissant dans ce domaine.

Si l'on examine, rapidement, une carte des 21 régions de la France métropolitaine, on s'aperçoit immédiatement que celles-ci ont des tailles très différentes. Ainsi la région « Nord-Pas-de-Calais » est-elle réduite à deux départements, «le Nord» et le «Pas-de-Calais», de même que l'Alsace avec le « Haut-Rhin » et le «Bas-Rhin ». Ces deux régions ont toutefois une très forte identité et ont d'ailleurs des politiques volontaristes de soutien à la recherche et à l'innovation. La Normandie, quant à elle se trouve divisée en deux régions : la Basse et la Haute-Normandie (avec respectivement trois et deux départements). Mais il existe aussi trois ou quatre régions qui, par leur taille et leur poids démographique, peuvent être comparées aux grands et moyens Länder d'Allemagne, comme l'Ile-de-France qui compte 11 millions d'habitants, les régions Rhône-Alpes (5 millions d'habitants) et Provence-Alpes-Côte d'Azur (4 millions d'habitants). Certes, le Land de Hambourg est petit, mais avec ses 2 millions d'habitants et son pouvoir économique il pèse lourd en Allemagne.... Très souvent donc, les régions françaises n'ont pas la taille critique pour mettre en œuvre une politique de soutien à la recherche et à l'innovation dont elles n'ont d'ailleurs pas la responsabilité.

\section{Un nouveau contexte mondial}

On est confronté depuis pratiquement deux décennies à un nouveau contexte mondial qui se caractérise par une internationalisation croissante de la recherche dans pratiquement tous les domaines. Ainsi, en France, 12\% de la dépense de recherche publique civile est-elle effectuée dans un système de référence européen à travers le Programme cadre de l'UE, le programme Eurêka, la douzaine d'organisations européennes de recherche comme le CERN et l'ESA. Il faut noter une autre donnée importante: $44 \%$ des publications françaises sont effectuées en co-publications internationales et $15 \%$ avec des auteurs allemands. La recherche, par ailleurs, est considérée, aujourd'hui, comme un facteur de 
compétitivité des entreprises. Ainsi les multinationales conduisent $28 \%$ de leur $R \& D$ à l'étranger (c'est-à-dire en dehors de leur pays d'origine). On assiste parallèlement, enfin, à une concentration croissante des moyens de la recherche avec un ancrage territorial : une région (ou un Land en Allemagne), une grande ville. D'où cette dualité croissante entre l'internationalisation de la recherche et les dynamiques territoriales. C'est un fait très important qui n'est pas propre à la France, qui s'accompagne d'une concurrence accrue entre les territoires pour attirer des investissements publics de recherche ainsi que ceux des entreprises, en particulier les firmes multinationales qui investissent pour créer des laboratoires en France, en Europe mais aussi, bien sûr, à l'échelle mondiale. D'où la nécessité, plus ou moins reconnue, de conduire des stratégies d'actions concertées entre les acteurs: l'Etat, les régions, les villes et les entreprises. Cette nécessité est reconnue dans son principe mais elle est loin d'être toujours mise en œuvre... en particulier en France.

Dans ce contexte, quelles évolutions la politique de la recherche et de l'innovation française a-t-elle connues récemment? Il faut souligner, d'entrée de jeu, que les questions que pose la concurrence internationale et donc la compétitivité sont au cœur du débat sur l'évolution du système de recherche et de technologie français; il en va certainement de même en Allemagne. La nécessité de faire évoluer les structures de recherche et de favoriser les relations entre les acteurs publics et privés, ainsi que l'importance du lien entre la R\&D et la compétitivité sont reconnues en France par la très grande majorité des acteurs du système. L'Association Nationale pour la Recherche et la Technologie (ANRT) a créé, en 2001, le programme de travail associatif sur la recherche et l'innovation FutuRIS pour analyser les forces et les faiblesses du système national et faire des propositions de réforme ; dans ce cadre, un groupe de travail sur "l'internationalisation des activités de R\&D et d'innovation et son impact sur les territoires 》 a réfléchi à ces questions et a remis un rapport qui a été publié en décembre 2007. Les questions de compétitivité étaient au cœur de ce rapport dont nous expliciterons les principales conclusions. Plus récemment, le Conseil d'Analyse Economique, qui est un conseil placé auprès du Premier Ministre, a publié, en juin 2008, un rapport très intéressant sur les régions et l'innovation qui aboutit à des conclusions très voisines. Signalons aussi les analyses d'un livre, La grande transition, publié au début 2008 par Pierre Veltz, un sociologue spécialiste des territoires, dont le titre indique bien que le développement économique, social, scientifique et technologique des territoires est un grand enjeu en France mais qu'il faut pour cela réaliser une grande transition qui est loin d'être faite... pour qu'un Etat à la tradition centralisatrice permette à la dynamique des acteurs locaux de jouer pleinement son rôle. Pierre Veltz est aujourd'hui le responsable de l'administration du Secrétariat d'Etat à la Région Capitale (dans le gouvernement actuel, un Secrétaire d'Etat chargé de la Région Capitale a la tâche de dynamiser la région Ile-de-France, l'aménagement et la dynamisation de cette région magmatique étant considérés comme un enjeu na- 
tional). Enfin, un certain nombre de réformes sont en cours sur lesquelles nous allons nous pencher.

\section{Un nouveau contexte français}

On a pris conscience peu à peu, en France, de la nécessité de faire évoluer le système de recherche et la politique d'innovation. Cette prise de conscience a, sans doute, été favorisée par la crainte des délocalisations industrielles, y compris celle de laboratoires de recherche industrielle dans les pays émergents comme la Chine et l'Inde où les firmes multinationales investissent vers la R\&D (comme le montre un récent rapport de l'OCDE sur la politique d'innovation en Chine). A cela s'ajoute la crainte d'un déclassement du système universitaire français que l'on pourrait appeler le «syndrome de Shanghai »: les universités françaises sont loin d'être dans le peloton de tête dans ce classement des universités mondiales réalisé par une équipe d'universitaires de Shanghai. Beaucoup d'universitaires français ont contesté ce classement en mettant en doute la pertinence des critères qui ont été choisis mais, dans leur for intérieur, ils se sont quand même interrogés sur la compétitivité internationale du système universitaire français, en particulier sa capacité à attirer des étudiants et des chercheurs étrangers. Par ailleurs la recherche universitaire française rencontre des difficultés pour recruter des universitaires étrangers de haut niveau, pour des raisons salariales, une situation que connaissent beaucoup moins d'autres pays européens.

Des initiatives ont été prises ces dernières années pour faire évoluer le système : des accords ont été signés dans les années 1980 entre les organismes de recherche comme le CNRS et les entreprises pour créer des laboratoires mixtes, la loi Allègre (1999) permet une plus grande mobilité des chercheurs et la création d'incubateurs dans les universités. Mais surtout des réformes institutionnelles sont intervenues dans les trois dernières années dans le cadre d'une nouvelle loi sur la recherche votée en 2006. C'est ainsi qu'a été créée une nouvelle agence, l'Agence Nationale pour la Recherche (ANR) qui est, dans une certaine mesure, l'équivalent de la DFG allemande (l'ANR finance des projets mais aussi des programmes et ne limite pas son intervention à la recherche académique). Des nouveaux modes de coopération entre partenaires ont été institués avec: les Pôles de Recherche et d'Enseignement Supérieur (PRES), des fondations de coopération scientifique, des Réseaux de Recherche Thématique Avancée (RTRA) dans des domaines comme les sciences sociales, la physique, les neurosciences (il y en a 13 environ et une dizaine en sciences médicales), les instituts Carnot (31 au total) qui se veulent l'équivalent des instituts Fraunhofer allemands (mais en sont un faible «ersatz» ...). La création, depuis 2005, des pôles de compétitivité associant entreprises et laboratoires de recherche publics sur des objectifs technologiques (formant ainsi des clusters) est une initiative importante qui vise à stimuler la compétitivité de 
l'industrie : 71 pôles ont été labellisés dont 17 sont mondiaux ou à vocation mondiale, avec environ 1,5 milliard $€$ de crédits qui ont été engagés sur la période 2005-2008. Enfin, il faut souligner que la loi sur l'autonomie des universités, votée en 2007, confère une autonomie aux universités dans bon nombre de domaines et en fait de véritables acteurs locaux et régionaux. Cette loi a été complétée en 2008 par un "plan Campus» qui, après un appel d'offres, prévoit à travers des contrats, accordés par le ministère de l'Enseignement supérieur et de la recherche, un plan de financement d'une dizaine d'universités (ou groupement locaux d'universités) pour développer et améliorer leurs infrastructures (sept universités en province et trois dans la région Ile-de-France, le plan a été étendu à une dizaine d'autres universités en 2009). Le budget total prévu par le plan campus s'élève à 5 milliards $€$ (provenant de la vente par l'Etat français de $10 \%$ de ses actions EDF). Le gouvernement allemand précédent, celui de Gerhard Schröder, avait pris une mesure analogue qu'a poursuivie l'actuel gouvernement. On a donc un certain parallélisme entre les préoccupations allemandes et françaises dans ce domaine: il s'agit de faire émerger des pôles universitaires ayant un pouvoir d'attraction international.

Si l'on s'attarde quelques instants sur une carte des pôles de compétitivité labellisés (qui reflète la situation en juillet 2007), on constate qu'il y a une douzaine de grands pôles et une soixantaine de "petits» pôles. Évidemment, on peut souligner que l'on a une très grande dispersion, ou un saupoudrage des moyens à travers des pôles localisés sur tout le territoire. Il est clair que certains pôles mobilisent un potentiel scientifique et technique qui est relativement peu important. Ainsi un «Pôle Enfant» a été créé à Cholet (dans la région Pays de la Loire), il travaille sur l'habillement et les jouets, un autre sur les industries et le pin maritime du futur dans les Landes, etc. Ces choix peuvent s'expliquer, en général, par une tradition industrielle et technologique locale. C'est le cas pour les deux pôles cités précédemment mais aussi, à Avignon, où l'INRA a créé dans une région agricole, il y a longtemps déjà, un institut de recherche sur les légumes et les fruits qui est l'un des meilleurs d'Europe avec toute une série de PME, des semenciers notamment, qui se sont installées à proximité de l'INRA et qui ont une vocation internationale. Sur ces bases un pôle de compétitivité sur les fruits et légumes y a été créé, ce n'est pas un pôle mondial mais son rayonnement international est d'ores et déjà certain. On remarque aussi la présence dans le Nord-Pas-de-Calais d'un pôle sur les textiles (Up-text) qui a pour vocation de mobiliser des entreprises pour mettre au point des textiles innovants à haute performance. Le Nord-Pas-de-Calais est une vieille région de tradition industrielle et ouvrière, notamment avec l'industrie textile qui était l'un de ses points forts mais qui a subi un fort déclin dans les années 1950/1960 : à Roubaix, à Tourcoing il existe ainsi quantité de friches industrielles (certaines d'entre elles sont transformées en centres de loisir ou de commerce, parfois en musées magnifiques d'ailleurs), mais certaines sont en train de remonter la pente; des en- 
treprises investissent dans des nouvelles techniques pour produire de nouveaux tissus. C'est l'objectif du pôle Up-text de transformer cette industrie.

Carte 1 : Les pôles de compétitivité français (en juillet 2007)

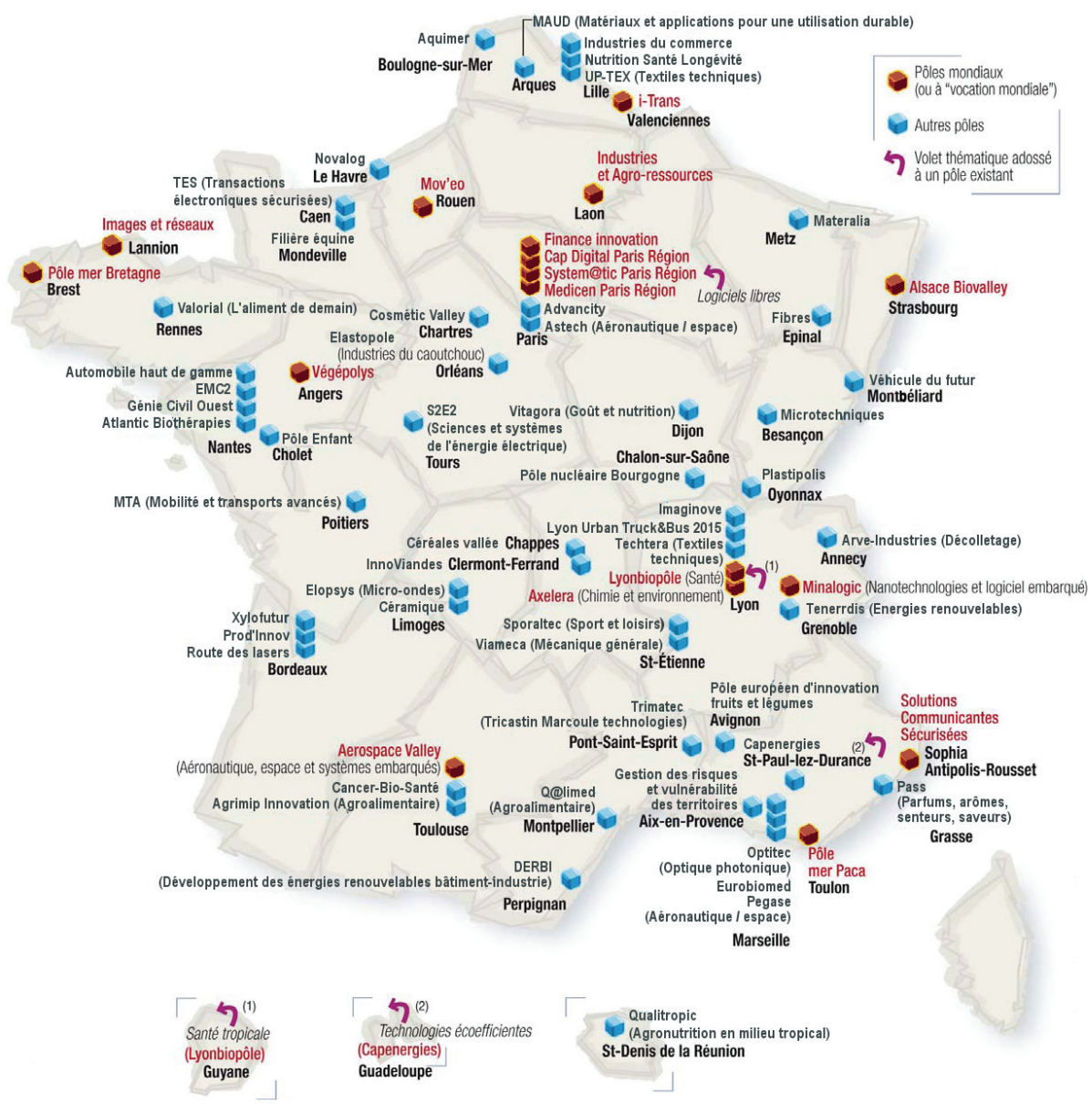

Source : DIACT.

Cette dispersion des pôles de compétitivité s'explique en grande partie par des vocations et des traditions industrielles qui pèsent dans les régions mais qui peuvent être un atout. L'avenir industriel ne se dessine pas nécessairement autour des technologies de pointe du triangle «technologies de l'information biotechnologies - nanotechnologies », des industries dites «traditionnelles» peuvent trouver un nouveau souffle technique grâce à la recherche et au progrès technique (c'est en particulier la thèse de l'historien des techniques britannique, David Edgerton dans son livre The Shock of the Old: Technology and Global 
History since 1900). Autrement dit la compétitivité ne peut s'apprécier seulement à l'aune des techniques dites de pointe, ce qu'oublient souvent les politiques d'innovation, mais c'est un tout autre débat.

\section{Attractivité et « compétitivité »}

On réalise, à partir d'exemples historiques, qu'il existe des facteurs d'attractivité pour un territoire qui permettent à une grande ville ou une région d'attirer des investissements de l'Etat ou des entreprises pour la R\&D et l'innovation, certains d'entre eux sont repérables par des indicateurs, et sur lesquels on peut penser que des politiques publiques peuvent jouer : politiques de l'Etat et des régions. FutuRIS a d'abord tenté d'identifier ces facteurs. Il y en a une trentaine, et l'évaluation de leur pertinence, très variable, a donné lieu à des publications et a fait l'objet de nombreuses thèses. Pour schématiser, on peut regrouper ces facteurs en grandes catégories dont nous ne mentionnerons que quelques unes : les facteurs humains (la qualité de la main-d'œuvre scientifique et l'ouverture internationale des chercheurs), les facteurs scientifiques et technologiques (l'existence de centres d'excellence de renommée internationale qui attirent les chercheurs par exemple), les facteurs liés aux régions (existence de réseaux d'entreprises, la tradition de coopération entre les entreprises et les universités, la politique d'aide régionale), des facteurs de marché (potentiel de croissance d'un marché local au sens large), les facteurs liés aux aspects financiers (le rôle de l'Etat dans le financement de la recherche, la fiscalité, le coût de la R\&D). Nombre d'études ont mis en évidence également l'importance de facteurs liés à l'environnement global de la R\&D : l'existence d'infrastructures locales (transports, télécommunications, cadre et qualité de vie, dynamisme du marché du travail, etc.). Autrement dit, pour une ville, l'existence d'un réseau de transport (TGV et avions) la mettant en relation avec les grandes métropoles européennes est un atout de poids, à l'inverse un relatif enclavement constitue un handicap sérieux (c'est le cas d'une grande partie du centre de la France). L'existence d'un tissu urbain dynamique est clairement un facteur d'attraction pour des universitaires, des chercheurs et des cadres d'entreprises. En France, des villes comme Lyon, Nantes, Montpellier, Strasbourg et, plus récemment Lille, ont réussi à redynamiser leur environnement et ont acquis un pouvoir d'attraction (à l'inverse dans le sud de l'Île-de-France, la région de Saclay, qui bénéficie d'importants investissements de recherche mais qui ont été construits dans un désert urbain au milieu de nulle part, peine à dynamiser son tissu scientifique et industriel). En France, la région de Grenoble est considérée comme l'archétype du cluster scientifique et industriel à succès car l'industrie et l'université ont l'habitude depuis un siècle de travailler ensemble, elle a, par ailleurs, l'avantage de bénéficier d'un cadre de vie attractif (la montagne). 
Au-delà de ce travail d'identification des facteurs d'attractivité qui peuvent peser dans des décisions de localisation d'activités de recherche, peut-on faire des constats à l'échelle de l'Europe ? C'est sans doute possible en utilisant des indicateurs quantitatifs. Ainsi, si on examine les 260 régions européennes, en utilisant les indicateurs de l'Observatoire des Siences et des Techniques (OST), on constate qu'en 2006, parmi les 30 premières grandes régions européennes pour la recherche et l'innovation figurent seulement trois régions françaises : l'Ile-de-France, Rhône-Alpes, Provence-Alpes-Côte d'Azur. En utilisant les indicateurs du European Scoreboard (qui a pris des villes) on trouve seulement trois villes françaises dans la liste des 30 grandes agglomérations de recherche et d'innovation européennes: Paris, Lyon, Toulouse. Les classements sont donc légèrement différents, les villes de la région ProvenceAlpes-Côte d'Azur (Marseille ou Nice) n'apparaissent pas dans le classement européen. Revenons sur le classement de l'OST qui, dans son rapport 2008, utilise un indicateur hybride (cf. tableau 1): publications scientifiques et brevets (comptés à part égale) qui figurent dans les bases internationales. Prenons, à titre d'exemple, le cas de l'Ile-de-France, supposons que celle-ci «pèse » $5 \%$ des publications dans la recherche en Europe (ce qui est à peu près le cas) et $7 \%$ des brevets, avec un indicateur hybride où les publications et les brevets ont le même poids, l'indicateur mixte de l'Ile-de-France serait donc de 6\%. En 2006, le poids européen de l'Ile-de-France était de 5,11\%, la deuxième région européenne est celle de Munich (avec un indicateur de 3,58\%, étant entendu que Munich correspond à l'Oberbayern), Stuttgart est numéro trois et Londres en quatrième position ; on trouve Rhône-Alpes en sixième position et ProvenceAlpes-Côte d'Azur à la $27^{\mathrm{e}}$ position. On doit remarquer que dans le classement de l'OST parmi les 260 régions européennes on trouve, avec les trois régions françaises, 12 régions allemandes parmi les 30 premières régions qui à elles seules, représentent $47 \%$ de l'activité scientifique et technique de l'UE. Bien entendu, on observera que la région Ile-de-France compte 11 millions d'habitants, et la "région » Oberbayern, environ 5 millions seulement. Il est donc intéressant de rapporter les activités scientifiques et techniques mesurées avec l'indicateur de l'OST à la population active (ce nouvel indicateur représentant en quelque sorte une " densité scientifique et technique ») et en faisant ce rapport, on constate alors que Munich, Stuttgart et Karlsruhe dépassent l'Ile-deFrance, Eindhoven étant la première région européenne devant Munich (la place de Eindhoven dans ce classement s'expliquant très largement par le poids de Philips). On observe aussi, grâce à ces indicateurs, la dynamique dans le temps : l'Ile-de-France augmente légèrement en densité scientifique et technique entre 2001 et 2006 alors que Munich et Londres baissent et que Provence-Alpes-Côte d'Azur, Stuttgart et Karlsruhe montent légèrement. Bien évidemment dans ce classement global certaines régions européennes montent, par exemple celles des régions des nouveaux Etats membres (il n'y en a aucune parmi les 30) on peut supposer que la région de Dresde va monter au cours de cette décennie car des 
investissements colossaux, en R\&D notamment, y ont été consentis par le gouvernement fédéral (Dresde occupe la $62^{\mathrm{e}}$ place dans le classement en 2006, elle était à la $67^{\mathrm{e}}$ place en 2001). Cette géographie régionale se modifie donc mais très lentement (la montée en puissance de la Catalogne en Espagne a ainsi été régulière, elle est au $15^{\mathrm{e}}$ rang devant Madrid, en 2006, qu'elle a nettement dépassée depuis 2001) avec des disparités qui, au cours du temps, se creusent ou dans certains cas s'effacent; il est probable que les facteurs d'attractivité jouent dans un sens ou dans un autre pour amplifier ce phénomène.

\section{Tableau 1 : Les trente premières régions européennes pour les activités scientifiques et techniques}

\begin{tabular}{|c|c|c|c|c|c|c|c|c|c|}
\hline \multirow[b]{3}{*}{ Région } & \multicolumn{9}{|c|}{ Activité S\&T } \\
\hline & \multicolumn{3}{|c|}{ Rong européen 2006 en } & \multicolumn{3}{|c|}{ Port UE $27(\%)$} & \multicolumn{3}{|c|}{ Densité par rapport ò la population active } \\
\hline & S\&T & $\begin{array}{l}\text { publica- } \\
\text { tions }\end{array}$ & $\begin{array}{c}\text { demandes de } \\
\text { brevet européen }\end{array}$ & 2001 & 2006 & $\begin{array}{c}\text { Évolution } \\
2006 / 2001(\%)\end{array}$ & 2001 & 2006 & $\begin{array}{c}\text { Évolution } \\
2006 / 2001(\%)\end{array}$ \\
\hline Îe-de-France (FRA) & 1 & 1 & 1 & 5,66 & 5,11 & .10 & 230 & 234 & +2 \\
\hline Région de Munich (DEU) & 2 & 8 & 3 & 3,58 & 3,02 & .16 & 376 & 313 & .17 \\
\hline Région de Stuttgart (DEU) & 3 & 61 & 2 & 2,48 & 2,57 & +4 & 285 & 288 & +1 \\
\hline Région de Londres (RU) & 4 & 2 & 21 & 2,87 & 2,52 & .12 & 178 & 156 & -12 \\
\hline Lombardie (ITA) & 5 & 5 & 10 & 2,11 & 2,07 & -2 & 115 & 109 & .5 \\
\hline Rhône-Alpes (FRA) & 6 & 7 & 9 & 2,04 & 2,00 & -2 & 179 & 177 & -1 \\
\hline Région de Cologne (DEU) & 7 & 12 & 5 & 2,05 & 1,97 & -4 & 236 & 219 & .7 \\
\hline Donemark (DNK) & 8 & 3 & 11 & 1,92 & 1,89 & -2 & 150 & 151 & +1 \\
\hline Région de Karlsruhe (DEU) & 9 & 17 & 8 & 1,72 & 1,81 & +5 & 298 & 304 & +2 \\
\hline Région d'Eindhoven (NLD) & 10 & 85 & 4 & 1,50 & 1,75 & +17 & 273 & 320 & +17 \\
\hline Région de Düsseldoor (DEU) & 11 & 36 & 7 & 2,02 & 1,62 & -20 & 191 & 150 & -21 \\
\hline Région de Darmstodt (DEU) & 12 & 46 & 6 & 1,90 & 1,58 & .17 & 227 & 190 & .16 \\
\hline Région d'Helsinki (FIN) & 13 & 14 & 14 & 1,55 & 1,39 & .10 & 259 & 237 & -9 \\
\hline Région de Berlin (DEU) & 14 & 11 & 19 & 1,38 & 1,32 & -5 & 180 & 172 & -4 \\
\hline Cotalogne (ESP) & 15 & 6 & 36 & 1,02 & 1,19 & +17 & 74 & 76 & +3 \\
\hline Région de Stockholm (SWE) & 16 & 16 & 18 & 1,59 & 1,16 & -27 & 352 & 258 & -27 \\
\hline Région de Madrid (ESP) & 17 & 4 & 79 & 1,07 & 1,12 & +5 & 93 & 82 & -12 \\
\hline Région de Tübingen (DEU) & 18 & 41 & 13 & 1,13 & 1,11 & -2 & 291 & 280 & -4 \\
\hline Émilie-Romagne (ITA) & 19 & 19 & 20 & 1,07 & 1,09 & +2 & 129 & 129 & 0 \\
\hline Région de Combridge (RU) & 20 & 10 & 43 & 1,38 & 1,09 & -21 & 278 & 224 & .19 \\
\hline Région d'Oxford (RU) & 21 & 13 & 34 & 1,37 & 1,08 & -21 & 265 & 207 & .22 \\
\hline Région de Fribourg (DEU) & 22 & 75 & 12 & 1,05 & 1,07 & +1 & 230 & 220 & -4 \\
\hline Région de Rotterdam (NLD) & 23 & 15 & 30 & 1,04 & 1,04 & -1 & 132 & 133 & +1 \\
\hline Région de Rome (ITA) & 24 & 9 & 66 & 0,96 & 1,01 & +5 & 98 & 103 & +5 \\
\hline Palatinat (DEU) & 25 & 56 & 15 & 1,16 & 1,00 & .14 & 273 & 230 & -16 \\
\hline Région de Nuremberg (DEU) & 26 & 78 & 16 & 0,93 & 0,93 & 0 & 248 & 248 & 0 \\
\hline Provence-Alpes-Côte d'Azur (FRA) & 27 & 24 & 29 & 0,91 & 0,90 & .1 & 115 & 118 & +3 \\
\hline Région d'Arnsberg (DEU) & 28 & 59 & 17 & 1,00 & 0,88 & .11 & 132 & 117 & -11 \\
\hline Région d'Amsterdam (NLD) & 29 & 18 & 46 & 0,82 & 0,84 & +2 & 137 & 138 & +1 \\
\hline Vénétie (ITA) & 30 & 38 & 25 & 0,74 & 0,82 & +11 & 81 & 87 & +8 \\
\hline Ensemble & & 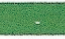 & & 50,00 & 46,90 & .6 & - & 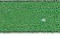 & . \\
\hline lonnees Thoms & & & & & & & & & 18 \\
\hline
\end{tabular}

Source : Rapport OST 2008. 


\section{Les dilemmes du jacobinisme : l'Etat et les régions face à la « compétitivité »}

Au-delà de ces constats globaux, le rapport FutuRIS a été amené à faire un diagnostic en trois points s'agissant des rapports entre politique nationale de recherche et d'innovation et les politiques régionales en France.

Le premier point du diagnostic de FutuRIS est que la traduction «territoriale » des stratégies des principaux acteurs publics nationaux (ministères, organismes publics, agences) est insuffisamment explicite. Cela signifie, d'une part, que l'Etat n'a pas de vision très claire de l'impact de ses priorités scientifiques et de ses programmes sur la compétitivité des territoires et que, d'autre part, le rôle que peuvent jouer les dynamiques régionales dans une politique de recherche et d'innovation n'est pas clairement envisagé. Faute d'une telle vision et d'une étroite concertation entre tous les acteurs, une stratégie nationale visant à promouvoir la compétitivité est difficile à mettre en œuvre. Le deuxième point du diagnostic complète le premier dans la mesure où l'on observe que l'explicitation souvent insuffisante des stratégies des acteurs nationaux, la fragmentation de leurs politiques dans certains domaines de recherche, voire parfois l'absence de cohérence sectorielle, sont un handicap sérieux pour toutes les actions d'accompagnement des dynamiques locales qui sont catalysées de plus en plus par les collectivités territoriales. A cela s'ajoute une absence de " visibilité » internationale des universités et des écoles d'ingénieurs qui est certainement pénalisante. Si les mécanismes nouveaux qui ont été créés récemment (Réseaux, ANR, PRES, etc.) ont un effet structurant, ils brouillent aussi la lisibilité du système national. Il en va de même des pôles de compétitivité qui, toutefois, ont le mérite de favoriser, malgré leur nombre, la convergence des stratégies nationales et régionales. Il faut compléter ce diagnostic en rappelant que si les lois de décentralisation de 1982 et de 2004 (la loi Raffarin de 2004 a complété de façon cosmétique la loi de décentralisation de 1982) n'ont transféré aucune compétence en matière de recherche et de d'innovation aux collectivités territoriales, celles-ci ont multiplié les initiatives de soutien à ces activités qui représentent des enjeux économiques importants en engageant des moyens financiers croissants. On constate aussi, toutefois, que les régions ont été conduites à mettre en œuvre de multiples dispositifs de soutien à la recherche et à l'innovation en réponse à des besoins régionaux, mais que leur complexité et leur manque de lisibilité et de synergie avec ceux des acteurs nationaux ne contribuent pas toujours à renforcer l'attractivité des territoires. Certaines régions ont pris conscience des carences de leur dispositif et ont créé, récemment, des « agences régionales de l'innovation » qui regroupent en leur sein toutes les procédures de soutien à l'innovation, en particulier dans les PME. Il y a donc une difficulté réelle de la stratégie nationale pour promouvoir la compétitivité des territoires mais, on l'a signalé précédemment, les pôles de compétitivité ont été créés pour tenter de pallier ce problème. Le troisième et dernier élément du diagnostic est le suivant : l'absence au niveau national d'une vision claire de la 
dimension européenne de la compétitivité (par exemple en application de l'agenda de Lisbonne) et de ses implications territoriales ne permet ni de mettre en œuvre des stratégies concertées d'acteurs publics et privés, ni d'utiliser au mieux les moyens européens. On constate ainsi que, contrairement à leurs homologues européennes, les régions françaises utilisent beaucoup moins les fonds structurels européens, les actions du Programme cadre et des prêts de la Banque Européenne d'Investissement (BEI) qui permettent de structurer les potentiels régionaux. Il existe aussi, bien sûr, des opérations multilatérales, comme 1'opération Biovalley entre 1'Allemagne, la Suisse et la France (une coopération dans la recherche biologique entre les villes universitaires de Strasbourg, Fribourg et Bâle), qui sont des exemples intéressants de coopération transfrontalière mais qui demeurent encore rares. Il n'en demeure pas moins que la dynamique européenne sur le champ de la technologie est insuffisante (le programme Eurêka lancé il y a 20 ans est sur une voie déclinante et l'Allemagne d'ailleurs ne s'y investit plus guère), mais il est vrai que le contexte régional pèse désormais d'un grand poids pour catalyser le développement technologique des entreprises, en particulier les PME.

Il faut sans doute apporter quelques correctifs par rapport à cette analyse de la prégnance du «jacobinisme» en France. La centralisation des pouvoirs politico-administratifs, une caractéristique du jacobinisme, n'est finalement pas aussi cartésienne qu'on le croit. Bon nombre d'acteurs publics ont su préserver ou acquérir une large autonomie. Ainsi le CEA a-t-il poursuivi avec continuité (et l'accord périodique du pouvoir politique) une stratégie nucléaire, qu'il a menée contre vents et marées, qui a conduit la France à renforcer son autonomie énergétique. La question nucléaire est un point de désaccord avec l'Allemagne mais les faits sont là. Plus généralement, la décentralisation est rentrée petit à petit dans les mœurs après la loi Defferre de 1982 et les régions, et dans une large mesure les collectivités, comme les départements et les communautés urbaines, ont su prendre des responsabilités nouvelles. Mais l'Etat doit admettre que la compétitivité suppose la mise en œuvre de dynamiques locales avec un large degré d'autonomie qui ont un effet catalytique. Cela il ne l'a pas encore admis quoi qu'il dise !

FutuRIS n'en est pas resté à des constats et a fait un certain nombre de propositions que nous allons présenter en les résumant. La première proposition s'adresse à l'Etat: l'efficacité d'une action visant à dynamiser le potentiel scientifique et technologique des territoires suppose à la fois une meilleure cohérence de cette action et une simplification du dispositif institutionnel avec une définition claire du rôle des acteurs et une délégation large des responsabilités. Ceci implique de donner une réelle autonomie d'initiative aux régions, en particulier en matière d'aide à l'innovation, avec, par exemple, une "régionalisation» partielle du Fonds Unique Interministériel qui soutient la recherche appliquée et l'innovation (placé sous la responsabilité de la Direction Générale des Entreprises qui dépend du ministère de l'Industrie) et des compétences 
régionales d'OSEO-Innovation, c'est -à-dire leur transfert aux régions. FutuRIS propose, dans le même ordre d'idées, une simplification du dispositif des pôles de compétitivité : l'Etat devant avoir la responsabilité des pôles dits à vocation mondiale (une douzaine au maximum), les régions ayant celle de lancer les pôles à vocation nationale (certains d'entre eux pouvant avoir une vocation européenne à long terme). Cette décentralisation des responsabilités serait facilitée par une nouvelle politique contractuelle associant Etat et régions. La deuxième catégorie de propositions s'adresse aux institutions régionales. Il est nécessaire de fédérer les efforts, en particulier ceux des acteurs locaux (centres de recherche, entreprises, universités) ; s'agissant des universités, l'autonomie, en application de la loi de réforme de 2007, est le moyen privilégié qui doit leur permettre de jouer un rôle catalytique en matière de recherche et de formation au plan régional, au besoin en contractant des «alliances ». Ainsi, par exemple, la fusion des trois universités de Strasbourg, le $1^{\text {er }}$ janvier 2009, est-elle déjà un acte fort qui va dans ce sens. FutuRIS propose également une meilleure gouvernance régionale pour éviter une prolifération de structures et d'outils et un saupoudrage des moyens d'une part en créant, par exemple, des agences régionales d'aide à l'innovation, et d'autre part en mettant en œuvre des moyens pour présenter une offre régionale "visible » et attractive aux entreprises afin de faciliter des implantations d'activités et la création de nouveaux laboratoires (ce que font d'ailleurs certaines régions). Le Comité d'Analyse Economique a proposé dans son rapport de créer, dans chaque région, une mission commune Etat-région pour toutes les actions de soutien à l'attractivité territoriale. C'est une proposition qui va aussi dans ce sens et qui a le mérite de favoriser le dialogue entre l'Etat et les régions. Le troisième groupe de propositions est plus spécifiquement international. Le rapport FutuRIS propose de lancer de façon périodique des actions concertées entre les organismes de recherche et les entreprises pour détecter et analyser les signaux de recherche sur des thèmes de recherche en émergence dans les laboratoires mondiaux. Il propose, par ailleurs une application réaliste du principe de « subsidiarité » pour que l'UE réserve ses interventions financières aux plateformes technologiques multinationales (via par exemple les «Joint Technology Initiatives ») et aux « Eurorégions ». Les régions françaises, enfin, devraient ainsi avoir la capacité de mobiliser davantage les fonds européens (les fonds structurels et les prêts de la BEI) pour le soutien à des actions de recherche et d'innovation.

DANS LA CONCLUSION D'UN ARTICLE SUR LES PÔLES DE COMPÉTITIVITÉ paru, en 2008, dans la revue Futuribles, Thierry Weil et Stéphanie Fen Chong s'interrogeaient : "Peut-on faire pousser une fleur en tirant sur sa tige ?». Se demandant quelle pouvait être la pertinence d'une évaluation des pôles de compétitivité à laquelle le gouvernement avait fait procéder deux ans après leur lancement effectif, ils soulignaient qu'il peut être utile de laisser jouer la dynamique du temps. Autrement dit, pour prendre une image de jardinage, il 
faut que l'Etat, s'il veut être jardinier, n'arrose pas n'importe comment, ne mette pas des engrais et des pesticides à contretemps, mais il doit laisser le temps au temps. C'est un point important qu'il faut retenir s'agissant des investissements de recherche et d'innovation : les actions pour favoriser l'attractivité et la compétitivité sont des actions de longue haleine (il en va certainement de même pour la construction européenne...) qui gagnent aussi à bâtir sur les traditions historiques de villes et de régions dans les domaines de la science, de la technologie et de l'industrie.

Il paraît utile de souligner aussi en conclusion qu'il faut sans doute procéder à un aggiornamento des politiques européennes de soutien à la compétitivité. Le soutien aux PME ne devrait pas être le rôle des programmes cadre et rarement, d'ailleurs, celui des programmes européens quoi qu'on dise, sauf s'ils concernent des opérations interrégionales ou transfrontalières qu'ils peuvent amplifier (du franco-allemand, du franco-belge, et même du franco-suisse puisque la Suisse est associée au Programme cadre). Il faut, en revanche, faire davantage jouer, à l'avenir, la coopération bilatérale pour des opérations entre régions voisines. Il en existe quelques unes, mais probablement en nombre insuffisant, entre la France et l'Allemagne, de même qu'entre la France et les pays voisins comme la Suisse, l'Italie, la Belgique et le Luxembourg, l'Espagne, voire le Royaume-Uni. C'est un point important pour faire jouer les dynamiques interrégionales à l'échelle de l'Europe. La concertation entre la France et l'Allemagne est certainement un catalyseur utile pour faire progresser la dynamique européenne dans tous ces domaines.

\section{Indications bibliographiques}

ANRT, FutuRIs, Le Système Français de Recherche et d'Innovation face aux défis de l'internationalisation des activités de Recherche Développement et innovation, FutuRis, Paris, 2007

CONSEIL D’ANALYSE ÉCONOMIQUE, Innovation et compétitivité des régions, La documentation française, Paris, 2008

LESOURNE J., RANDET D., La recherche et l'innovation en France, Odile Jacob, Paris, 2008

LESOURNE J., RANDET D., La recherche et l'innovation en France, Odile Jacob, Paris, 2007

OBSERVATOIRE DES SCIENCES ET DES TECHNIQUES (OST), Indicateurs de sciences et de technologies 2008, Economica, Paris, 2008

Veltz P., La grande transition, Le Seuil, Paris, 2008

WeIL T., Fen ChONG S., « Les pôles de compétitivité français », Futuribles, 342, juin 2008, p. 5-26. 
- III -

CLUSTERS ET RÉSEAUX TERRITORIAUX 



\section{Les transferts de technologies en France}

\section{Thierry WEIL}

L'innovation, associée à la recherche, est essentielle à la croissance économique d'un pays. Encore faut-il que les entreprises puissent bénéficier des résultats de la recherche académique via les transferts de technologies. La contribution de Thierry Weil, professeur associé au Centre d'économie industrielle de l'Ecole des mines de Paris, impliqué dans l'Observatoire des pôles de compétitivité et le projet 'Epictete' de l'ANR, présente les grandes caractéristiques du cadre institutionnel et les évolutions récentes de la coopération science/industrie.

Les pôles de compétitivité en France sont une politique beaucoup plus récente qu'en Allemagne. De plus, le transfert de technologie est rarement ce qui apparait en premier quand plusieurs organisations commencent à travailler ensemble. Il est donc un peu tôt pour observer des transferts dans les pôles réellement nouveaux. Je vais donc évoquer un peu plus généralement les problèmes liés aux transferts de technologies entre le monde académique et la recherche industrielle en France.

Je vais d'abord rappeler quelques notions sur la recherche académique, la recherche industrielle, l'innovation qui posent le problème du transfert de l'un à l'autre et des évolutions récentes, puis entrer dans le cœur du sujet, c'est-à-dire la coopération entre la recherche académique et l'industrie en France. Je vous donne directement la conclusion qui est qu'au niveau global, nous sommes très mauvais. C'est un constat qui a été notamment établi il y a deux ans dans un rapport coordonné par un inspecteur général des finances et un inspecteur de l'éducation nationale, Henri Guillaume et Jean-Richard Cytermann. Je ne pense pas que la situation ait énormément changé. Ce qui a changé sans doute, c'est la prise de conscience qui fait qu'on s'en préoccupe plus. Du coup, il y a eu un certain nombre d'initiatives récentes et prometteuses. Pour ne pas vous laisser sur une image trop pessimiste, j'ajouterai que si la situation globale peut sembler désespérante, il y a cependant beaucoup de réussites sur le terrain et d'expériences très intéressantes. Je dirai bien sûr un mot des pôles de compétitivité, bien qu'il soit trop tôt (puisque les pôles ont été lancés il y a trois ans et que beaucoup se sont véritablement mis en place il y a un an ou deux) pour citer des success stories de transferts de technologies qui soient vraiment imputables à la création des pôles.

\section{Innovation et croissance}

Il y a un consensus maintenant clair sur le fait que l'innovation est l'un des principaux moteurs de la croissance, en tout cas dans les pays développés où 
l'on est obligé, pour que les consommateurs achètent quelque chose, de leur offrir de nouvelles fonctionnalités. C'est aussi, et ce point est relativement nouveau, un des facteurs importants pour accéder aux marchés des pays émergents. Autrefois, on revendait des Mercedes d'occasion dans les pays d'Afrique du Nord après les avoir utilisées dix ans en Europe. Maintenant, on conçoit en Inde des voitures en fonction des spécificités du marché indien. Et pourtant, on constate qu'un certain nombre d'entreprises réduisent leur budget de $R \& D$, parce que le lien entre la recherche industrielle et l'innovation n'a malheureusement rien d'automatique. Si les entreprises savent qu'elles ont besoin d'innovation, ont-elles besoin de recherche? Est-ce que la recherche va les rendre riches? La recherche consiste à dépenser de l'argent pour avoir des idées. Ce dont les industriels ont besoin c'est plutôt d'innovation, c'est-à-dire exactement l'inverse : transformer des idées en revenus. On trouve certes une corrélation entre le fait que les gens dépensent, font de la recherche, et le fait qu'ils sont riches, mais le sens de la causalité éventuelle n'est pas clair. Bell Labs ou IBM faisaient énormément de recherche quand elles bénéficiaient d'un quasi-monopole ; quand elles ont affronté une concurrence plus rude, elles ont fait moins de recherche. La plupart des actionnaires et des parties prenantes, attendent que l'entreprise crée plus de richesses qu'elle n'en consomme. Il y a beaucoup de moyens pour y arriver, l'innovation n'étant que l'un d'entre eux. Il n'est pas évident de convaincre les industriels que c'est par l'innovation et en particulier par la recherche qu'ils créeront le plus de valeur.

L'impact des efforts de recherche publique sur l'innovation des entreprises dépend également de très nombreux facteurs. Pourtant les entreprises ont plus que jamais besoin de trouver dans leur environnement des compétences complémentaires aux leurs. Si autrefois, beaucoup d'entreprises étaient très intégrées et voulaient tout faire elles-mêmes, parfois selon l'expression anglosaxonne, jusqu'aux vis et aux boulons (bolts and nuts) dont elles avaient besoin pour produire, c'est de moins en moins le cas : elles achètent leurs vis, trouvent des partenaires pour des boulons sophistiqués et en ce qui concerne les idées, elles les ont le plus souvent en interagissant avec le monde académique, avec leurs fournisseurs, voire avec leurs concurrents. C'est-à-dire que si on peut gagner de l'argent avec ses propres bonnes idées, on peut aussi en gagner avec celles des autres, qui coûtent souvent moins cher à récupérer.

Certains se demandent même si on a encore besoin de R\&D dans l'industrie et des consultants sont allés jusqu'à conseiller de passer de la « recherche et développement» au «pillage et développement». Les travaux plus récents montrent qu'on a besoin d'une activité de recherche, car pour être capable d'utiliser la R\&D des autres, il faut pouvoir comprendre ce qui se passe à l'extérieur et dans le monde académique, et pour cela la meilleure manière, c'est d'avoir soi-même une culture technique, base d'une capacité d'absorption des développements réalisés ailleurs. 


\section{Les formes de transferts technologiques}

Je n'insiste pas plus sur les problèmes du management de l'innovation dans l'industrie et j'arrive au sujet d'aujourd'hui, c'est-à-dire la valorisation par les entreprises du potentiel de la recherche académique. Je me fonde sur le rapport que j'ai déjà cité de Jean-Richard Cytermann et d'Henri Guillaume qui distinguent quatre modalités de transfert entre le monde académique et l'industrie : les contrats de recherche (où des laboratoires académiques travaillent à la demande d'un industriel pour apporter des réponses aux problèmes de cet industriel ou développer les connaissances nécessaires), les licences de brevets, la création d'entreprises et la mobilité des chercheurs. Cette mobilité peut correspondre au passage d'un chercheur du monde académique à l'industrie, voire l'inverse, ce qui est plus rare en France, ou être temporaire ou partielle, par des relations de conseil par exemple.

\section{Une organisation historique de la recherche inadaptée}

J'en viens donc à mon premier point: la situation globale en France est peu satisfaisante, en partie pour des raisons historiques. Le système de recherche français était traditionnellement organisé autour de grands programmes de souveraineté nationale (défense, nucléaire, spatial), réalisés dans des organisations spécifiques, avec moins de financements sur la science plus quotidienne (physique du solide, informatique, biologie) qui est celle qui nourrit les start-up dans d'autres pays. Dans le monde académique, faire de la recherche finalisée ou appliquée n'est toujours pas bien considéré. Les réformes récentes vont dans le bon sens sur beaucoup de points, mais pas sur tous : elles ne résolvent pas le problème du manque de coordination entre le pilotage de la recherche publique assuré par le ministère de la Recherche et de l'Enseignement supérieur et le soutien à l'innovation des entreprises, assuré par le ministère de l'Economie.

On juge souvent la France très inefficace en constatant qu'elle fait un effort public de recherche très important $(1 \%$ du PIB). C'est en effet plutôt plus que les pays comparables et à peu près autant que les Etats-Unis. Mais cet effort de $\mathrm{R} \& \mathrm{D}$ était très concentré sur quelques secteurs et grands défis (c'est un héritage de la période gaullienne), avec beaucoup d'argent consacré au nucléaire, à la fois civil et militaire, au spatial, à l'aéronautique, aux transports. Cet argent n'a pas été dépensé inefficacement, mais si on regarde ce que j'appelle la $R \& D$ civile-civile, c'est-à-dire hors grands programmes, on est à peu près à $0,6 \% \mathrm{du}$ PIB. C'est un peu moins que ce que fait l'Allemagne, qui investit comme nous beaucoup sur l'aéronautique, mais dépense beaucoup moins notamment sur le nucléaire et le militaire. Donc notre effort de R\&D publique n'est pas dans les domaines qui conduisent le plus facilement aux créations d'entreprises 'civiles', notamment là où se font le gros des start-up américaines (les biotechnologies et l'informatique, voire l'électronique). On a donc une organisation historique 
devenue assez inadaptée avec notamment une université très sous-dotée : un étudiant de l'enseignement supérieur universitaire coûte beaucoup moins cher en France qu'en Allemagne, et ce n'est pas parce que l'on serait plus efficace. L'université est privée de très bons chercheurs par la concurrence des grands organismes de recherche où ils n'ont pas d'obligations d'enseignement et de très bons étudiants par le fait que l'enseignement professionnalisant est surtout réalisé dans les grandes écoles d'ingénieurs ou de gestion, les IUT (qui dépendent de l'université mais en sont physiquement séparés) ou les écoles d'agriculture. Les organismes de recherche, pour des raisons historiques que je ne vais pas développer ici, ont été paralysés par des arbitrages financiers qui ont conduit à créer de nombreux postes de chercheurs au détriment des moyens de recherche à la disposition des personnes qui bénéficiaient de ces postes ainsi que par une absence d'arbitrage stratégique entre les domaines, en tout cas depuis les années 1970.

Ce manque de pilotage est particulièrement pénalisant : dans les années 1990, le budget de la NASA a été divisé à peu près par deux. C'était après la chute du Mur de Berlin, on sortait de l'ère de la Guerre Froide et les priorités n'étaient pas les mêmes. Pendant le même temps, celui du NIH (le National Institute of Health) faisait plus que doubler. Dans la même période en France, le budget du CNES est resté stable : on ne s'est pas aperçu que le Mur de Berlin était tombé et que la menace soviétique avait quelque peu changé de nature. En revanche, les budgets de l'INSERM n'ont que peu augmenté, et l'ensemble des recherches biomédicales en France ne représentent qu'environ $10 \%$ du budget du NIH.

On peut également évoquer une certaine confusion entre l'autonomie des universitaires et l'autonomie des universités. L'autonomie des universités est ce qui permet à l'université d'avoir une stratégie, de décider par exemple que l'Université de Poitiers sera leader en histoire médiévale, dût-on y faire des sacrifices en physique quantique ou en biochimie. Ce n'est évidemment pas la liberté des universitaires parce que le professeur de biochimie peut souhaiter que l'université ait une stratégie différente.

\section{Modernisation de la recherche en France...}

Plusieurs réformes se sont succédées récemment pour pallier ces déficiences du système de recherche national. La réforme de 1999 consiste en une loi sur l'innovation qui facilite la mobilité des chercheurs et qui crée une fiscalité plus favorable à la création d'entreprises. En 2005, un Pacte pour la recherche se traduit par la création de deux agences, une pour la recherche académique et une pour l'innovation industrielle. En 2006, une loi pour la recherche complète le Pacte pour la recherche et crée un certain nombre de nouvelles institutions de pilotage, d'évaluation, de fédération de moyens des universités et en 2007 enfin, la loi «liberté et responsabilité des universités» modifie la gouvernance des 
universités et donne notamment des pouvoirs de gestion beaucoup plus étendus aux présidents d'université en matière de ressources humaines, de recrutement et d'immobilier. Pour le pilotage, en revanche, on est resté chacun chez soi, chacun étant d'une part le ministère en charge de la Recherche et l'autre partie étant le ministère de l'Industrie et les ministères techniques (équipement, santé, défense, agriculture,...). On a créé une instance pour conseiller le gouvernement sur la politique de recherche : le Haut conseil pour la science et la technologie, placé auprès du Président de la république. Il a d'abord fallu lutter pour accoler le mot technologie car certains auraient préféré un Haut conseil pour la science. Bien qu'on ait obtenu dans le nom et dans les statuts de ce conseil qu'il ait une vision d'ensemble, le chef de l'Etat de l'époque a préféré privilégier l'aspect académique, en y nommant en grande majorité de grands scientifiques « nobélisables », mais qui n'ont pas toujours une conscience très aiguë des besoins de l'économie et des besoins de la société. Malgré ce handicap, les membres du Haut conseil font cependant du bon travail mais ils sont très peu écoutés. Ce Haut conseil a produit deux rapports dès ses six premiers mois d'existence. A ma connaissance, il n'a depuis été donné aucune réponse à ces rapports et aux recommandations pourtant très claires qu'ils formulaient.

\section{...et nouveaux acteurs institutionnels}

Du côté de la recherche, on a créé l'Agence Nationale de la Recherche (ANR), qui privilégie l'excellence des projets, avec une assez faible prise en compte de leur impact économique, et dont les experts souhaitent recevoir un grand nombre de propositions, de manière à pouvoir être très objectifs et très sélectifs. Ils vont donc prendre un sujet à l'intitulé large pour avoir au moins quarante propositions et en sélectionner une petite dizaine.

Mais le système de jugement par les pairs avec parfois plusieurs niveaux successifs de sélection fait que les propositions originales ont peu de chances d'aboutir. J'ai eu l'occasion d'examiner récemment les programmes francoallemands dans le domaine de la pile à hydrogène. En France, ce programme était géré par l'ANR et avec le système que j'ai décrit, on sélectionnait les gens pour leur excellence scientifique, sans chercher à savoir si l'équipe qui coordonnait la recherche avait de bonnes connexions avec des entreprises qui pourraient exploiter les résultats. Dans le même temps, le partenaire allemand, le Projektträger Jülich qui, lui, travaillait par délégation du ministère fédéral de l'Economie, raisonnait différemment. Au lieu de dire : « on regarde votre projet, on le sélectionne ou non et on ne discute pas du contenu», il n'hésitait pas à dire : « ce projet n'est pas encore très bon, mais on va l'améliorer parce qu'il est porté par un industriel susceptible de jouer un rôle important pour développer la filière ». Pour Jülich et le Bundesministerium für Wirtschaft, l'objectif est qu'à la fin, l'Allemagne dispose d'industriels compétents dans ce domaine. Au besoin, les examinateurs encourageaient les porteurs de projets à changer de 
partenaires, à s'allier avec de meilleurs laboratoires et avec de meilleures équipes plutôt que de sélectionner sur l'excellence initiale, sans considération pour l'impact industriel.

A l'opposé de l'ANR, il y a la Direction Générale des Entreprises (DGE, aujourd'hui DGCIS) qui ne veut pas s'encombrer de l'agenda des chercheurs et agit dans une certaine ignorance de ce que peut faire la recherche académique. Tout cela ne facilite pas le couplage entre les orientations de la recherche publique et les entreprises. Il faut aussi mentionner l'Agence d'Evaluation de la Recherche et de l'Enseignement Supérieur (AERES). Cette nouvelle agence d'évaluation, selon les missions que lui confère la loi, est censée prendre en compte toutes les missions du chercheur, qui incluent la valorisation de ses travaux, la participation à l'expertise publique, la diffusion de la science dans des milieux plus larges que des simples étudiants, une participation aux débats avec la société. Cependant, quand on regarde ce qui se pratique aujourd'hui, l'AERES (c'est peut-être un problème d'âge et de mise en place car il s'agit d'une institution très jeune) ne regarde que les productions académiques au sens le plus restrictif et le plus traditionnel. Donc un laboratoire qui consacrerait de gros efforts à essayer de répondre à une demande industrielle ou à une demande de la société le ferait au détriment du jugement que l'AERES portera sur lui, au moins à court terme. Car le rapport Cytermann montre aussi que les laboratoires qui font plus de recherche contractuelle publient plus que les autres, donc que la recherche contractuelle ne se fait pas au détriment de la qualité académique mais y contribue souvent. Néanmoins, quand on fait la recherche contractuelle, momentanément, on publie moins et donc, au moins dans l'immédiat, on perd des points pour son évaluation.

En ce qui concerne l'image globale du financement de la recherche et de l'innovation en France en 2008, on voit dans la figure ci-contre, où la taille des carrés est proportionnelle aux budgets, une certaine disjonction entre la recherche académique à long terme financée sur crédits publics (en haut à gauche) et, en bas à droite différentes mesures d'appui à l'innovation, notamment le Crédit d'Impôt Recherche (diapositive ci-contre, C ANRT, FutuRIS, 2008).

Je ne vais pas vous laisser sur cette vision pessimiste, car il se passe quand même des choses encourageantes. J'ai mentionné en 1999 la loi sur l'innovation, en 2006, la création des instituts Carnot, calqués sur le modèle de la Fraunhofer Gesellschaft mais avec des taux d'abondement beaucoup plus faible. C'est de la Fraunhofer Gesellschaft « diluée ». Malheureusement, je ne suis pas sûr que le modèle supporte une dilution trop forte. Mais tout cela est relativement récent, donc on n'en voit pas encore beaucoup d'effets macroscopiques. La loi sur l'innovation de 1999 a principalement autorisé les enseignants-chercheurs et les chercheurs à demander un congé sabbatique pour créer leur entreprise si celle-ci valorisait leurs travaux, à faire de la consultance, à prendre des participations dans les entreprises valorisant leurs travaux, voire à siéger à leur conseil d'administration. 


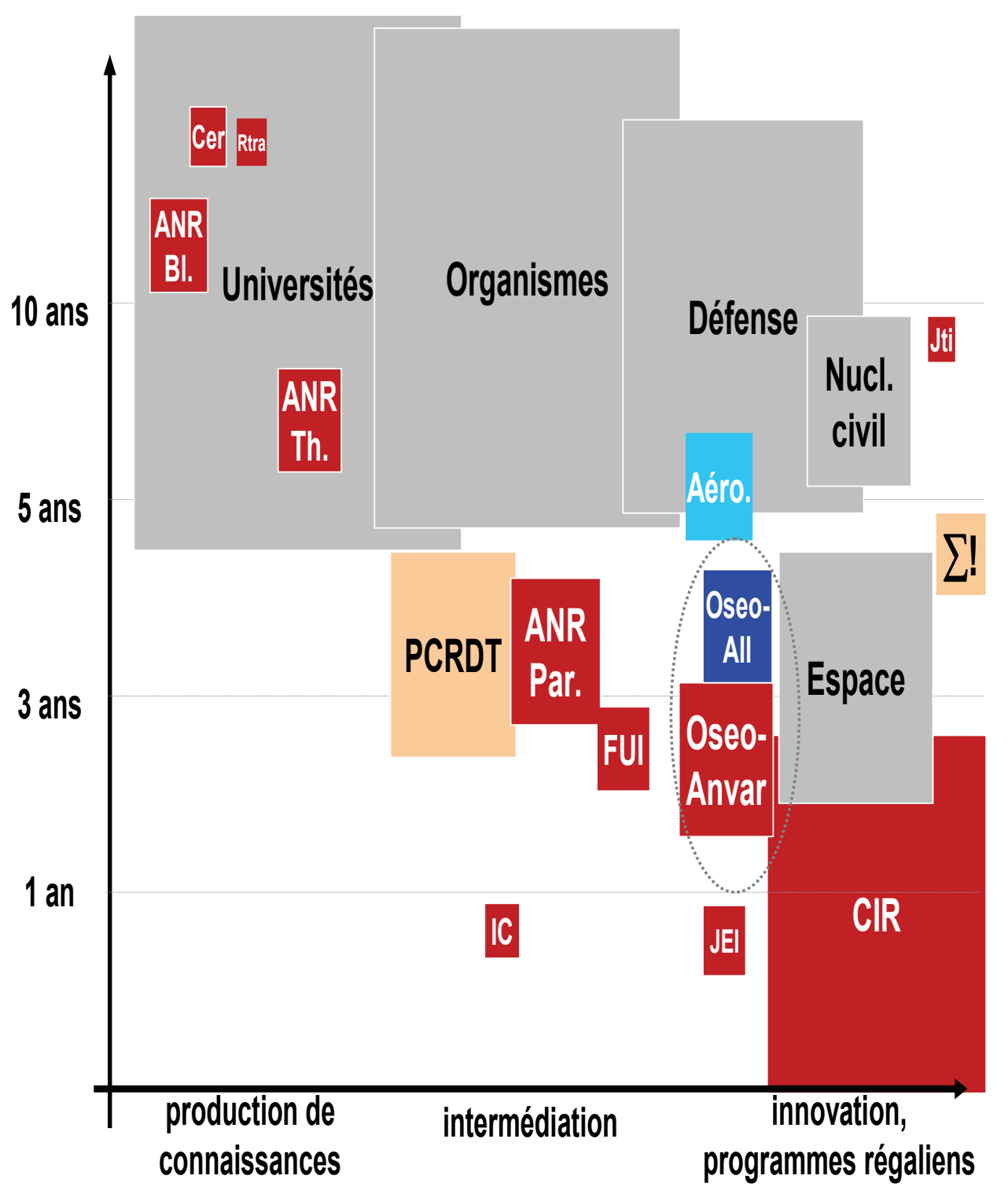

Légende : (la plupart des dispositifs cités sont décrits dans le corps de l'article),

CER : Conseil Européen pour la Recherche (ERC), RTRA: Réseaux Thématiques de Recherche Avancée, ANR: Agence Nationale de la Recherche (programmes «blancs, thématiques ou partenariaux), JTI : plates-formes technologiques européennes (Joint Technology Initiative), $\sum$ !: programme Eureka, PCRDT: Programme Cadre européen de Recherche et Développement Technologique, FUI : Fonds Unique Interministériel (géré par le ministère de l'industrie), OSEO : agence chargée de soutenir l'innovation industrielle, IC : Instituts Carnot, CIR : crédit d'impôt accordé aux entreprises qui font de la recherche, JEI : mesures fiscales en faveur des jeunes entreprises innovantes, «Défense, Aéronautique, Nucléaire civil, Espace » : grands programmes technologiques. 
La loi a également établi des règles plus incitatives de partage des redevances de propriété industrielle au sein des universités et des organismes publics. Elle a créé un certain nombre d'incubateurs et elle a encouragé la création de SAIC (Services d'Activités Industrielles et Commerciales) des universités, ce qu'on appelle dans le monde anglo-saxon des « offices of technology transfer ».

\section{Aide à la création d'entreprises}

En ce qui concerne les incubateurs, il faut savoir (c'est une réalité assez mal connue) que les Français créent des entreprises. Pour 1 milliard $€$ investis dans la recherche publique en France, il y a 18 créations d'entreprises, alors qu'il y en a 11 aux USA. Je crois que l'Allemagne est un peu entre les deux. Donc on sait créer les entreprises, le problème réside dans ce qui se passe après, au niveau des perspectives de développement de ces jeunes entreprises créées. Les incubateurs dits "Allègre » de 1999 ont eu des destins très différents. Certains ont bien fonctionné (le critère de succès est qu'un certain nombre d'entreprises ont pu faire des levées de fonds auprès de venture capitalistes ou entrer en bourse) d'autres pas du tout, avec deux types de problèmes. Le premier concerne le personnel de ces incubateurs. Très souvent, on en a confié la direction à un universitaire que ses cours ennuyaient et qui parfois connaissait des théories sur l'innovation, mais un créateur de start-up a aussi besoin qu'on l'aide à négocier un petit délai avec les impôts ou l'URSSAF lorsque la trésorerie est tendue. Les incubateurs qui fonctionnaient plutôt bien étaient pilotés par les personnes qui avaient une expérience personnelle d'entrepreneur. Certains incubateurs ont aussi été victimes de contrats de performance avec des indicateurs quantitatifs où l'incubateur s'engageait par exemple dans les deux ans à accueillir 20 entreprises : par conséquent ils ont cherché à faire du nombre.

Ce que j'ai constaté quand j'ai examiné quelques incubateurs il y a quelques années, c'est qu'il y avait trois types d'entreprises dans les incubateurs dont un seul aurait dû s'y trouver. Il y avait d'abord des entreprises extrêmement performantes, qui n'avaient aucun besoin des services de l'incubateur. Quand je demandais à leurs responsables ce qu'ils faisaient dans l'incubateur, ils me disaient que cela leur permettait de bénéficier d'un chèque-service de $30000 €$, d'un loyer dans des conditions très avantageuses (partout ailleurs, on leur demanderait d'énormes cautions ou un loyer beaucoup plus cher) et d'une image valorisante, mais qu'ils n'utilisaient pas les services de l'incubateur. Ces entreprises font monter les statistiques de succès de l'incubateur, mais l'incubateur leur apporte une faible valeur ajoutée puisqu'elles s'en seraient très bien sorties avec une simple caution. La deuxième catégorie est constituée de projets qui séduisent un comité de sélection trop majoritairement composé de chercheurs académiques, mais qu'un venture capitaliste n'aurait jamais sélectionnés parce que l'équipe est fragile ou que le projet est beaucoup trop loin des applications... Enfin, la dernière catégorie est la cible légitime du dispositif, 
c'est-à-dire des personnes qui portent un projet encore fragile et qui ont besoin de quelques mois, voire un an ou deux pour le renforcer et être suffisamment préparées pour aller voir des venture capitalistes. Celles-ci devraient normalement composer $100 \%$ de la population de l'incubateur.

\section{Valorisation de la recherche}

Deuxième aspect, les services de valorisation des universités et des organismes. La situation est très contrastée. Certains organismes de recherche ont des performances tout à fait intéressantes, qu'il s'agisse de création de start-up, de redevances de licences ou de contrats avec des entreprises, notamment le CEA et l'INRIA, ou les universités de Strasbourg et Grenoble; d'autres sont beaucoup plus fragiles. Le CNRS était satisfait de ses résultats, mais l'essentiel de ses revenus provenait de deux molécules découvertes par le professeur Pierre Potier, les principes actifs du Taxotère ${ }^{\circledR}$ et de la Navelbine ${ }^{\circledR}$, qui tombent d'ailleurs dans le domaine public incessamment [le Taxotère est entré dans le domaine public en janvier 2010 ; NDA]. Il est tout à fait courant que dans un portefeuille de brevets, $90 \%$ des revenus viennent de $20 \%$ des brevets, mais pour le CNRS ce n'était pas $20 \%$, mais $0,2 \%$, c'est-à-dire ces deux brevets. Le reste des revenus, environ 4 millions $€$, ne couvrirait même pas les frais de fonctionnement des services chargés de gérer les licences. Et à l'université, c'était traditionnellement encore pire, sauf quelques brillantes exceptions : Strasbourg et Grenoble ainsi qu'une partie de Paris XI ou de Paris VI.

\section{Le financement public de la recherche industrielle}

Les instituts Carnot sont très récents : ils ont été créés par la loi de 2006. Ils sont totalement inspirés de la Fraunhofer Gesellschaft, dont je rappelle le principe : quand un industriel accorde $1 €$ de contrat à un laboratoire public, la Fraunhofer Gesellschaft, le Land et l'Etat fédéral abondent en général d' $1 €$ chacun. Le cycle vertueux est que l'institut Carnot ou la Fraunhofer Gesellschaft facture au vrai coût ses prestations de recherche, mais l'abondement qu'il reçoit lui permet de faire de la recherche en amont donc de générer de nouvelles connaissances intéressantes pour les entreprises. Cela, c'est l'image d'Epinal, et certains trichent un peu, profitant des autres revenus pour ne faire payer à l'industriel qu'une partie seulement du coût de sa recherche. Mais, même dans ce cas, c'est intéressant car cela réduit la barrière à l'entrée pour l'entreprise et stimule donc les interactions entre entreprises et laboratoires publics. C'est pourquoi MM. Guillaume et Cytermann préconisent cette voie, consistant à donner une incitation aux partenaires publics, sachant que le partenaire privé, quant à lui, récupérera un meilleur accès à la recherche publique, dans de meilleures conditions financières ou avec des personnes très motivées pour avoir des contrats, puisqu'elles ont d'autres bénéfices induits par les contrats. Ce n'est pas 
ce que fait le Fonds Unique Interministériel (FUI) qui finance dans le cadre des pôles de compétitivité les projets communs entre industriels et universitaires, en distribuant la subvention à tous les partenaires. Dans le cadre du Crédit d'Impôt Recherche (CIR), quoi que fasse l'industriel, il a une réduction d'impôts proportionnelle au budget qu'il engage et celle-ci est doublée pour la partie de ce budget confiée à des institutions publiques. Rappelons que le rapport de Guillaume et Cytermann indique que le financement public de la recherche privée en France est l'un des plus importants de la zone OCDE, et cela avant même l'augmentation très substantielle du CIR.

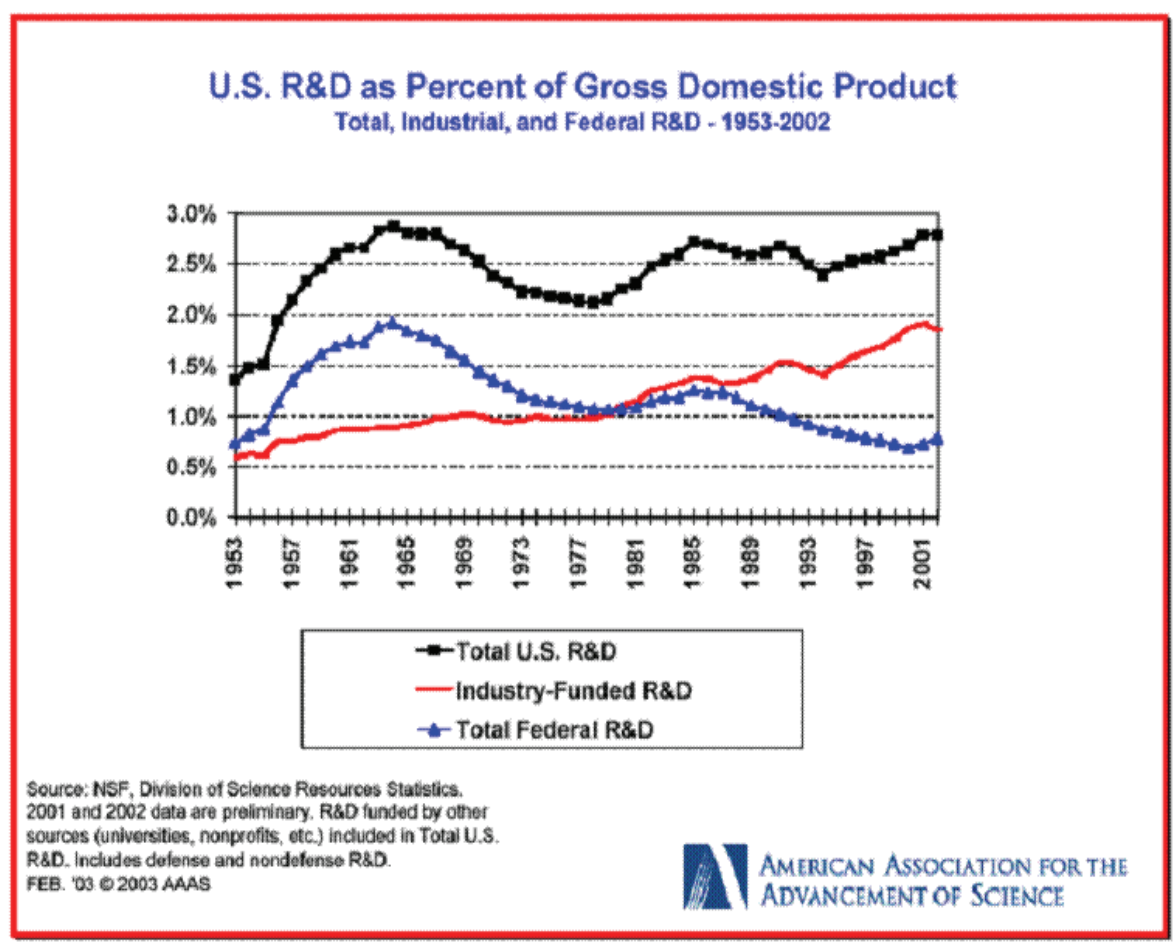

Cela dit, le processus permettant aux entreprises de bénéficier des investissements dans la recherche publique est très lent. On dit toujours que cela fonctionne beaucoup mieux aux Etats-Unis, mais avant d'arriver à la situation actuelle où les entreprises américaines consacrent deux fois plus de moyens à la recherche que le secteur public et confient des contrats importants aux universités et autres laboratoires publics, il y a eu 30 ans d'investissements publics très substantiels, puis encore 30 ans d'efforts soutenus. Ceux-ci ont démarré dans les années 1950 en partant d'une situation où l'Etat comme les entreprises ne consacraient chacun qu'un modeste $0,5 \%$ du PIB à la $R \& D$, pour atteindre au milieu des années 1960 une dépense publique de R\&D de 
près de $2 \%$ du PIB. La dépense des entreprises n'a rattrapé la dépense publique qu'au début des années 1980. La situation actuelle très favorable, où les Etats-Unis sont dans une situation proche de ce qui est l'objectif de Lisbonne pour les Européens, c'est-à-dire 1\% du PIB de financement public et $2 \%$ de financement privé de $\mathrm{R} \& \mathrm{D}$, est donc le résultat relativement récent d'une longue histoire. Il y a eu d'abord une lente accumulation de capital de connaissances en 30 ans d'investissements persistants dans la R\&D publique. Les industriels qui investissaient pour appliquer cette science ont pu en profiter. L'industriel mettait le dernier dollar, et comme la science est cumulative, récupérait le bénéfice de l'ensemble. Effectivement, à partir de 1980, l'investissement de R\&D industrielle est apparu très rentable aux entreprises et le mouvement s'est ensuite entretenu de lui-même.

Des études économétriques montreraient que quand on augmente l'investissement dans la $R \& D$ publique, cela se traduit par un effet sur la $R \& D$ industrielle seulement six ans après. Or on est en train de vouloir tirer les leçons de ce que font les pôles de compétitivité au bout de moins de trois ans. Donc si on n'observe rien, au moins dans le domaine des résultats quantitatifs mesurables, ce n'est pas forcément qu'il ne se passe rien. Malgré les nombreuses mesures destinées à accélérer le processus, à stimuler les phénomènes qui ont permis l'apparition de clusters ailleurs, la construction d'un écosystème efficace reste lente et demande de la persévérance. Il faut tempérer un peu son impatience.

\section{Sucess stories de transferts de technologies}

Même si j'ai donné une vision globale pessimiste, il se passe sur le terrain de nombreuses choses très intéressantes, mais très localisées, c'est-à-dire qu'il y a dans la Gaule romanisée, quelques petits villages d'Astérix qui résistent encore et toujours à l'envahisseur et à la norme commune, mais qui sont très isolés. Ces 3\% de villages gaulois réalisent $75 \%$ de la recherche sur contrats. Ce sont des exceptions plus ou moins malfamées dans notre beau jardin à la française de la recherche académique. Parmi les exceptions de longue date, il y a l'Institut Pasteur qui n'a que $40 \%$ de subventions de l'Etat, le reste venant des redevances de licences sur des vaccins ou de services. C'est bien connu maintenant puisque notre dernier prix Nobel était en train de travailler avec l'Institut Pasteur du Cambodge, au moment où elle a reçu son prix, à rendre des services non lucratifs d'aide aux systèmes de santé locaux. C'est également l'Institut Pasteur qui a l'un des incubateurs les plus performants, ayant permis à beaucoup d'entreprises qui se portent bien aujourd'hui de prendre leur envol.

On retrouve aussi des instituts Carnot, qui ont été labellisés instituts Carnot après coup mais qui ont tout de même une histoire assez longue. On mentionnera notamment le LETI, le laboratoire d'électronique du CEA, qui a 
créé beaucoup de start-up et dont l'activité est liée en majeure partie à l'industrie. Le LETI est d'ailleurs engagé dans des collaborations ambitieuses avec la Fraunhofer Gesellschaft. On peut encore citer Armines, l'association qui regroupe les centres de valorisation des écoles des mines, qui a fait en sorte que la moitié des ressources de recherche de l'Ecole des mines de Paris vienne de contrats, d'entreprises mais aussi de l'Union européenne, et qui gère un assez gros portefeuille de brevets et de logiciels.

De la même manière qu'il n'y a pas un modèle unique de politique de soutien à la recherche appliquée en Allemagne, le modèle français est assez foisonnant et divers quand on regarde de près. Il y a beaucoup de mécanismes destinés à stimuler les acteurs qui veulent travailler ensemble. Outre les instituts Carnot dont nous venons de parler, qui ont une spécialisation thématique mais ne privilégient pas les partenaires locaux, on mentionnera les "systèmes productifs locaux ", politique destinée à encourager les districts industriels traditionnels ou émergents, les Pôles de Recherche et d'Enseignement Supérieur (PRES) favorisant la construction de campus interdisciplinaires en permettant à divers établissements d'enseignement supérieur de regrouper leurs forces, les Réseaux Thématiques de Recherche Avancée (RTRA), plus tournés vers l'excellence dans la recherche, les Centres de Recherche, d'Innovation et de Transfert Technologique (CRITT), plutôt destinés à la recherche appliquée et aux PME, et enfin les pôles de compétitivité qui associent entreprises et recherche.

\section{Pôles de compétitivité}

On a donc beaucoup de combinaisons, certaines fonctionnant de manière satisfaisante et d'autres un peu moins, la dernière politique en date étant les pôles de compétitivité qui ressemblent un peu aux Kompetenznetze allemands. C'est tout de même quelque chose d'assez compliqué à faire fonctionner. A l'intérieur du pôle, on essaie de faire travailler ensemble des petites entreprises et des universités localisées sur les territoires, avec d'autres acteurs ayant des activités sur le territoire mais dont les centres décisionnels peuvent être ailleurs, comme les filiales de grandes entreprises ou les sites de recherche d'organismes nationaux.

Pour légitimer l'existence du pôle tant vis-à-vis des participants que des pouvoirs publics, les organisateurs du pôle se sont concentrés les premières années sur la mise en place de projets de recherche collaboratifs. C'était ce que l'Etat finançait et qui permettait à la structure de gouvernance d'un pôle d'être légitime vis-à-vis des tutelles et vis-à-vis de ses membres, puisque les projets retenus permettaient d'apporter des financements immédiats aux membres. Les pôles de compétitivité les plus mûrs commencent à se consacrer à des tâches nécessaires pour améliorer la compétitivité du territoire à plus long terme comme de construire une stratégie commune des membres, 
d'essayer de veiller à disposer des compétences requises pour réaliser cette stratégie sur le territoire en jouant sur la formation, d'attirer des investisseurs, etc. Dans les quelques success stories précédemment évoquées il y en a deux qui sont dans les pôles de compétitivité : le LETI, qui fait partie du pôle de compétitivité Minalogic, et les recherches sur la sécurité routière entre le centre de robotique de l'école des mines et Valeo qui se retrouvent dans le cadre des pôles de compétitivité System@tic, mais à partir de contrats qui existaient depuis très longtemps.

ENCORE UNE FOIS, LE TEMPS DE DÉVELOPPEMENT de tels succès font que ceux qui sont visibles sont le résultat d'une histoire de collaboration antérieure à la création du pôle, mais que l'existence du pôle a parfois stimulés. J'ai bon espoir qu'on constate dans quelques années des effets du mouvement créé par la mise en place des pôles et la multiplication de coopérations inédites et prometteuses. 



\title{
Réseaux d'innovation en Allemagne Principes généraux et exemples pratiques de Rhénanie du Nord - Westphalie
}

\author{
Petra Moog, Sebastian HanNY
}

Malgré une intégration économique mondiale de plus en plus profonde, la dimension locale s'impose depuis les années 1990 via la notion de " glocalisation » : l'ouverture des entreprises à l'international s'accompagne d'un ancrage régional fort, facteur d'une compétitivité accrue. Petra Moog et Sebastian Hanny* \# s'intéressent dans la présente contribution aux réseaux d'innovation régionaux allemands, et plus spécifiquement aux initiatives de promotion des réseaux en Rhénanie du Nord-Westphalie.

\section{Introduction}

C'est surtout en période de crise économique que les processus d'innovation sont souvent considérés comme une solution universelle aux problèmes, voire un remède miracle. L'innovation peut alors occuper des niveaux très divers. L'innovation régionale ou nationale, mais aussi l'innovation au plan international, semble toujours plus décisive pour le développement économique d'une branche ou d'un pays. L'utilisation ciblée et répandue du potentiel intellectuel pour produire des innovations ${ }^{1}$ dans la formation actuelle de la société du savoir ${ }^{2}$, afin de garantir et d'augmenter la croissance économique ainsi que le bien-être social, gagne donc régulièrement et notablement en importance (cf. Heidenreich, 2004). A l'heure actuelle, les économies basées sur le savoir attribuent $70 \%$ de la croissance économique au progrès technologique (cf. OCDE, 2003). Il n'est donc pas surprenant que les acteurs politiques aussi bien qu'économiques n'imputent pas les données alarmantes sur l'efficacité technologique ${ }^{3}$ de l'Allemagne (cf. DIW, 2004) seulement au manque de potentiel innovant du système de recherche économique, mais aussi à celui du sys-

\footnotetext{
* Petra Moog est titulaire de la chaire d'économie générale d'entreprise, spécialité succession d'entreprises à 1'Université de Siegen; Sebastian Hanny dirige le réseau de création d'entreprises des universités de Dortmund à l'unité de transfert de connaissances de l'Université technique de Dortmund.

\# Nous remercions la Fondation Volkswagen pour avoir soutenu le projet de recherche « The Transfer of Knowledge and Innovation From the University to the Economy ». Le présent article est un « coproduit» des recherches menées dans le cadre de ce projet subventionné.

${ }^{1}$ Définies ici comme la mise en œuvre d'une idée dans des produits commerciaux nouveaux ou améliorés, dans des processus opérationnels de l'industrie ou du commerce, ou bien dans de nouvelles formes de prestations de services (cf. OCDE, 1999).

${ }^{2}$ Pour la définition du terme, cf. Böhme/Stehr (1986); Stehr (1994).

${ }^{3}$ D'après le BMBF, l'efficacité technologique s'exprime par «de nouveaux produits et procédés innovants qui s'imposent sur les marchés internationaux ». Ils s'appuient sur la richesse inventive des entreprises allemandes et de l'utilisation économique des résultats de la recherche publique (cf. http://www.technologische-leistungsfaehigkeit.de [consulté le 06/09/10]).
} 
tème de recherche universitaire. Pour modifier cette situation, il faut changer les données institutionnelles dans le but de fournir des résultats plus transférables et exploitables, c'est-à-dire de rendre les universités et leurs chercheurs plus « entrepreneuriaux » ou plus orientés vers le management. C'est un changement souhaitable pour pouvoir garantir la performance de l'Allemagne dans la concurrence internationale d'une manière durable et favorable à l'intérêt général.

Pour cela, il faut des réseaux interactifs regroupant des représentants de divers contextes organisationnels, régionaux et pratiques. Les experts considèrent la société du $\mathrm{XXI}^{\mathrm{e}}$ siècle comme une société de réseaux (par ex. Becker et al., 2007). Les réseaux sont de plus en plus le symbole d'un transfert réussi de connaissances, de technologie et d'innovation (cf. Heidenreich, 2000; Kowol/Krohn, 1995; 2000; Rammert, 2003). ${ }^{4}$ Sous cette forme, le transfert de connaissances et de technologies (TCT) est souvent considéré comme le moyen de promouvoir l'économie au niveau national et régional (cf. par ex. Fritsch et al., 2007). Pour cela, il doit exister des systèmes d'innovation nationaux sur lesquels on puisse construire des systèmes d'innovation régionaux. En termes de succès du TCT, les systèmes d'innovation (régionaux) qui sont considérés comme particulièrement productifs et dynamiques sont ceux dans lesquels la science, l'entreprise et le monde de la finance sont liés mutuellement par diverses structures d'organisation en réseau, qui se concentrent sur de nouveaux thèmes de recherche dans le domaine des technologies de pointe et qui travaillent dans des secteurs différents des spécialités classiques (cf. Peter, 2003). Dans ce processus hétérogène de production de savoir et de TCT, la recherche universitaire joue un rôle important : elle est considérée comme l'élément central du « système d'innovation national » (cf. Soskice, 1997 ; Etzkowitz/Leydesdorff, 1997), voire comme le moteur de l'économie (cf. Etzkowitz, 2002). Dans cette perspective, la question des conditions optimales pour une recherche universitaire fructueuse parce que tournée vers l'innovation, dont le potentiel serait exploitable de manière plus extensive pour la production d'innovations, revêt une importance capitale pour la puissance économique d'une nation (au niveau national et régional; cf. Mowery/Rosenberg, 1999; Spielkamp et al., 2002; BMBF, 2003). En effet, ces innovations d'origine universitaire, nécessitant d'importantes recherches, représentent les fondations de nombreux usages commerciaux (sous forme de marchandises ou de services) et conquièrent des marchés très divers (cf. Schumpeter, 1942). Une "intégration de la science dans l'indus-

\footnotetext{
${ }^{4}$ Nous désignons sous le terme transfert de connaissances et d'innovation toutes les formes de transmissions d'idées, de connaissances, de capacités et d'expériences liées aux innovations d'un objet/sujet à un autre. Les moyens de transfert sont tous les types de contacts, des plus institutionnalisés aux plus informels, écrits ou oraux. Ce processus est itératif et repose sur des systèmes d'acteurs interdisciplinaires (cf. Etzkowitz/Webster/Healey, 1998).
} 
trie » n'est donc pas à séparer d'une «intégration de l'économie dans la science $»$ (cf. Weingart, 2001).

Pour le développement économique communal et régional, la mondialisation et le renforcement de la concurrence internationale imposent de nouvelles contraintes. Ainsi, depuis quelques années on constate que la mondialisation génère non seulement une compétition accrue entre les entreprises, mais aussi une concurrence toujours plus rude entre les sites. En conséquence, la promotion économique a entamé un changement de perspective: de la promotion d'entreprises individuelles, on est passé à la promotion de la région en tant que lieu d'activités économiques. Dans ce contexte, la promotion de clusters économiques est considérée comme un instrument essentiel. ${ }^{5}$ Dans son ouvrage sur l'avantage concurrentiel des nations, Michael Porter a posé en 1998 les fondations d'une politique moderne de promotion économique vouée au développement de clusters. Depuis, le concept de cluster s'est imposé comme instrument de promotion économique. "Pour que les résultats de la recherche se transforment en produits commercialisables plus rapidement, nous devons renforcer les réseaux qui relient les entreprises entre elles et les institutions de recherche avec les entreprises. C'est ce que nous allons faire. Ainsi vont naître des clusters sectoriels régionaux et technologiques nationaux qui renforcent la compétitivité de la région industrielle de Rhénanie du NordWestphalie. » (Rüttgers, 2005). Par conséquent, la suite de cet article présente plus en détail les réseaux d'innovation régionaux de Rhénanie du NordWestphalie.

Les avantages supposés d'un lieu qui entraînent la création ou l'existence de clusters économiques couronnés de succès sont principalement liés à l'existence de structures de réseau internes aux clusters. Cela a souvent amené les acteurs de la promotion économique à mettre sur pied des réseaux d'innovation pour soutenir et développer les structures régionales de clusters. De tels réseaux doivent organiser plus efficacement les transferts de connaissances de la recherche vers la pratique par une coopération plus étroite entre les entreprises et les institutions de recherche, afin d'activer la naissance d'innovations, d'apporter une contribution efficace au développement de la structure sectorielle régionale et enfin de résister à la concurrence mondiale. On espère, en encourageant ces réseaux d'innovation dits régionaux, c'est-àdire des réseaux dont l'objectif est de générer des innovations dans et pour une région, contribuer efficacement au développement de l'économie régionale. On peut toutefois se demander dans quelle mesure les structures de réseau qui favorisent le développement de clusters, telles qu'elles sont

\footnotetext{
${ }^{5}$ On définit les clusters comme « une concentration d'entreprises interconnectées, de fournisseurs spécialisés, de prestataires de services et d'institutions associées (universités, associations commerciales)» (cf. http://fr.wikipedia.org/wiki/Cluster [consulté le 06/09/10]). Dans le contexte des clusters économiques, la notion de prestataires de services inclut également les bailleurs de fonds, les conseillers fiscaux, les experts en création d'activités indépendantes et les avocats d'affaires.
} 
décrites dans la théorie, peuvent effectivement être influencées par la politique et la promotion économique, et où se trouvent les limites et les facteurs de succès du travail en réseau.

\section{Principes théoriques d'une politique économique (régionale) tournée vers l'innovation}

Avec la mondialisation, la thèse selon laquelle les entreprises agissent de plus en plus sans se préoccuper de leur localisation et que les régions et leurs caractéristiques locales perdent de l'importance pourrait prévaloir. Cette thèse fait toutefois face à la notion de « glocalisation»: le retour aux caractéristiques et aux offres régionales signifie que les entreprises peuvent améliorer leur compétitivité non seulement par l'intégration territoriale, mais aussi par une intégration sociale (via les réseaux) dans la région. Pour expliquer ce phénomène, la littérature spécialisée propose quatre théories: les districts industriels (Marshall, 1966), la théorie des clusters de Porter (1998), les milieux innovants (Camagni, 1991) et les régions apprenantes (Florida, 1995). Ces théories sont brièvement décrites ci-après pour mieux classer les exemples pratiques et les intégrer dans le contexte des analyses régionales des résultats.

Districts industriels : la théorie de Marshall (1966) a été redécouverte dans le cadre de la recherche sur l'innovation à dominante territoriale. Le cœur de cette théorie est formé par les petites et moyennes entreprises (PME). Une autre caractéristique des districts industriels est la concentration des entreprises d'une région sur une branche ou un type de production ou de services. Dans cette situation, chaque entreprise se spécialise dans des secteurs de la production industrielle ou des services et agit avec beaucoup de flexibilité (spécialisation flexible, Piore/Sabel, 1984) par rapport à la demande individuelle. Ainsi, les entreprises peuvent réagir très rapidement aux évolutions dynamiques sans frais d'équipement élevés. Outre ce type de partage très efficace du travail, la concurrence entre les entreprises est un autre facteur de succès des districts industriels. La proximité et souvent une étroite collaboration entraînent une bonne connaissance des autres entreprises. Les innovations de l'une sont très vite prises en considération par d'autres et entraînent des réactions plus rapides qui conditionnent elles-mêmes des innovations dans les processus de production ou les produits (services), ce qui encourage l'arrivée de nouvelles entreprises et la disparition (rapide) de celles qui ne s'adaptent pas aux conditions du marché. Les districts industriels se caractérisent par leur haut niveau de communication et de coopération, la confiance et la solidarité en tant que facteurs locaux subjectifs. Les inconvénients peuvent apparaître lorsque la confiance ou la proximité est exploitée et le réseau malmené. Un exemple remarquable de district industriel est toujours la région d'Emilie-Romagne dans le nord de l'Italie, dont les petites et moyennes entreprises ont tissé et tissent une structure semblable à un réseau (Teiljohann, 1994). 
Théorie des clusters : grâce à la théorie de Porter (1998), les réseaux, les coopérations et les maillages d'acteurs sont entrés dans la recherche macroéconomique (sur la croissance). La théorie des clusters étend l'idée des districts industriels en y ajoutant les aspects de minimisation des coûts et de création de valeur. Ces deux éléments peuvent être justifiés par des innovations externes à l'entreprise. Par ailleurs, les effets des institutions et de l'interaction des acteurs sont modélisés. Les hypothèses de base de Porter pour justifier l'existence d'un cluster sont : a) les entreprises et institutions d'une région, par ex. des fournisseurs ou clients spéciaux, les prestataires de services ainsi que l'université et les institutions gouvernementales, doivent être en relation. b) Il doit exister une concentration ou une délimitation territoriale dont l'extension n'est pas spécifique et qui peut varier dans le temps (village, ville, jusqu'au niveau international). c) Les acteurs doivent entretenir des relations qui peuvent être fondées soit sur l'échange ou la coopération, soit également sur la concurrence et la compétition ; c'est-à-dire qu'il existe des réseaux sociaux. Ces deux conceptions (les districts industriels et les clusters) en arrivent à la conclusion que la dimension territoriale et donc la région représentent un élément pertinent pour assurer un avantage compétitif durable. Ces avantages de la localisation reposent sur des coûts de transaction moindres et des innovations plus rapides et plus fortes qui se propagent et sont adoptées plus rapidement parmi les acteurs en raison de la proximité géographique et des relations étroites qu'ils entretiennent.

Milieu innovant : le concept élaboré par Camagni (1991) part du principe que les produits et services innovants viennent d'entreprises innovantes installées dans des régions innovantes. Les études empiriques montrent que les régions innovantes naissent d'elles-mêmes, c'est-à-dire de manière autonome. Les facteurs qui encouragent la formation de régions innovantes sont les universités et la présence de parcs technologiques (subventionnés par l'Etat). L'hypothèse de Camagni suppose cependant que les interventions publiques ne peuvent pas générer obligatoirement l'apparition d'innovations. La politique d'innovation peut alors tourner dans le vide, c'est-à-dire qu'on ne peut pas créer une «Silicon Valley» par une intervention publique. A elle seule, l'édification d'un parc technologique à proximité d'une université ne suffit pas à faire apparaître un climat innovant, et encore moins des entreprises obligatoirement innovantes. Un facteur important qui peut favoriser cette évolution est la dimension sociale, souvent négligée dans le processus économique. La loyauté, la confiance et l'éthique marquent les rapports entre les acteurs de l'économie qui devraient être intégrés dans une région (Granovetter, 1985) pour que les réseaux d'innovation soient fonctionnels et utiles. Ce processus se déroule au sein d'une région entre divers acteurs à l'intérieur de leur réseau qui repose ou est organisé sur des contacts personnels et une proximité géographique. Le milieu innovant apprend alors dans la collectivité, l'interaction des acteurs étant le moteur de l'innovation. 
Régions apprenantes: dans ce concept (tourné vers l'action) également (Florida, 1995), la mise en réseau des acteurs régionaux et/ou locaux est placée au premier plan, en particulier celle des petites et moyennes entreprises. Au final, toutes les conclusions des précédentes hypothèses se regroupent ici et constituent la base du processus d'apprentissage au sein d'une région. En raison des différentes relations qui unissent les entreprises et les autres acteurs (coopération ou concurrence) et de leur confrontation permanente, le processus de développement connaît de nombreuses rétroactions. Il en résulte le maintien et l'apparition de réseaux flexibles toujours en communication. Cela crée des expériences d'apprentissage au niveau individuel des acteurs ainsi qu'au niveau régional, ce qui peut être utile pour la région. Cela exige des régions et des acteurs de grandes capacités d'organisation et de flexibilité, car des concepts et des acteurs désordonnés et dispersés doivent être rassemblés de manière pertinente et sans cesse réorganisés pour maintenir les réseaux dans un état optimal. L'objectif est d'accélérer la dynamique régionale de développement. Contrairement au concept du milieu innovant, dans ce contexte les mesures politiques (publiques) sont considérées comme utiles pour faire avancer et influencer le développement régional (Stahl/Schreiber, 2003).

Ces quatre thèses interprètent différemment les possibilités de succès de l'intervention publique dans l'économie au moyen de concepts tournés vers l'innovation. On peut cependant leur trouver un dénominateur commun : une aide initiale de la part des institutions publiques est largement souhaitée, par exemple sous la forme d'un système national d'innovation ou d'une réglementation nationale destinée à encourager le transfert et l'entrepreneuriat (cf. projet Volkswagen). Toutefois, cette aide doit se limiter à la promotion du cadre institutionnel (par ex. systèmes d'incitation, lois). Ou bien il faut une aide ciblée et ponctuelle en faveur de la création d'un réseau d'acteurs et d'institutions (régionaux). En effet, si les régions disposent déjà d'une bonne infrastructure, mais ne sont pas fonctionnelles pour une génération optimisée des innovations, cela tient généralement au manque de coordination entre les acteurs et les éléments présents. La promotion doit donc engendrer la capacité à utiliser des potentiels jusqu'alors sous-développés grâce à la constitution de réseaux. Les mesures de promotion ayant des motifs politiques doivent ainsi stimuler le potentiel existant et contribuer à son épanouissement (Koschatzky, 2003). C'est pourquoi il apparaît que consacrer des moyens publics à lancer des processus de création de réseaux régionaux tournés vers l'innovation est une mesure ciblée et efficace ; cependant, cela doit toujours se faire dans l'esprit d'une mesure de soutien et d'incitation, non d'une contrainte (Fritsch, 2004). C'est avant tout la coopération des acteurs économiques, scientifiques et institutionnels qu'il faut encourager. Les ressources doivent être couplées, coordonnées et focalisées.

En fin de compte, il n'existe donc aucun instrument universel pour promouvoir l'innovation. Par contre, l'interaction et la mise en réseau des acteurs sont 
très importantes pour le processus d'innovation. En outre, une promotion adaptée individuellement à la région concernée semble nécessaire. D'une manière générale, on peut conclure (Fritsch, 2004) que :

- les ressources d'innovation régionales peuvent être activées et renforcées au moyen d'une promotion ciblée. Dans ce contexte, le capital humain est une ressource particulièrement importante, ce qui met au premier plan l'extension des possibilités de formation et des offres de qualification ;

- les ressources doivent être couplées et coordonnées dans des réseaux d'innovation régionaux. Pour cela, il est utile d'identifier les réseaux essentiels, dont la constitution devrait être accompagnée et soutenue financièrement. Cela peut se faire en encourageant les projets d'alliance et de coopération, en intégrant explicitement et en exploitant la proximité territoriale ;

- la création d'interfaces entre les réseaux d'innovation régionaux et les acteurs nationaux et internationaux est utile.

Apparemment, l'élan initial, l'édification, le soutien des réseaux d'innovation régionaux et une promotion orientée sur le long terme et la durabilité semblent donc constituer une mesure adaptée pour exploiter plus efficacement les potentiels de génération d'innovations existant dans les régions, pour motiver et mettre en marche les processus qui mènent à la formation de régions innovantes et à l'installation d'entreprises innovantes. L'infrastructure disponible semble alors utilisée plus efficacement.

\section{Réseaux d'innovation régionaux}

Avant d'aborder au dernier chapitre les exemples de réseaux d'innovation régionaux, voici tout d'abord une brève présentation de la notion.

D'une manière générale, les réseaux d'innovation régionaux peuvent être définis par une coopération entre entreprises et institutions impliquant plus de deux acteurs. L'objectif commun doit être la création et la mise en œuvre d'innovations (durables). En règle générale, les coopérations qui s'établissent dans le réseau sont peu formalisées; il se produit des échanges mutuels et des processus d'apprentissage où les acteurs du réseau se comportent de manière coopérative, tout cela étant souvent basé sur une orientation de projet à court ou moyen terme. Le plus souvent, des réseaux planifiés (artificiellement) se créent en raison de mesures régionales ou fédérales de promotion économique, afin d'influencer positivement le développement de structures sectorielles régionales et l'économie dans son ensemble (Becker et al., 2007; Fritsch et al., 2007; Stifterverband für die Deutsche Wissenschaft, 2007).

Les facteurs moteurs de la création, de l'établissement et du maintien à long terme de réseaux d'innovation régionaux sont tout d'abord la recherche d'un 
espace économique régional attractif. Ensuite, pour que ces réseaux existent, il est indispensable de savoir que les innovations ne sont pas un processus linéaire, mais nécessitent un partage du travail entre différents acteurs, pas des inventeurs isolés ou des entrepreneurs "schumpetériens », et que ces acteurs génèrent des innovations plus efficacement s'ils sont organisés en réseau. Les réseaux d'innovation régionaux résultent donc fréquemment de l'exigence d'un transfert efficace entre l'économie et la science.

\section{Schéma 1 : Formes de réseaux régionaux}

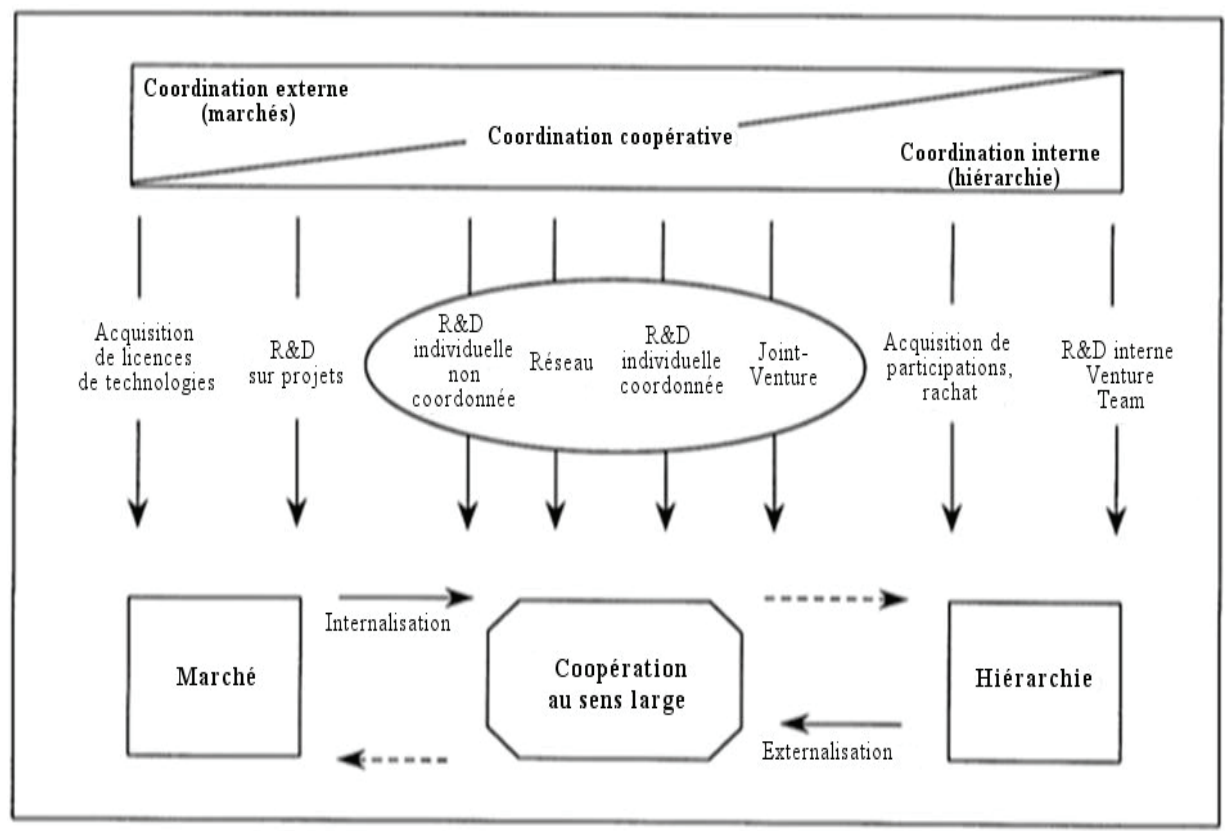

Source : Haritz, 2000.

Les systèmes d'innovation régionaux doivent compléter les institutions que sont le marché et les organisations (par ex. les entreprises ou les universités), ce qui permettra de réunir des chaînes de création de valeur jusqu'alors séparées et de les rendre plus efficaces (Burger-Menzel, 2006). On s'attend à ce que les réseaux régionaux connaissent la meilleure réussite s'ils ont auto-organisés et coordonnés par des acteurs autonomes (par ex. Haritz, 2000). De cette manière, les réseaux d'innovation régionaux se représentent entre le marché et la hiérarchie comme au schéma 1 .

En règle générale, les réseaux d'innovation régionaux sont intégrés dans les systèmes d'innovation nationaux, dans le cas de l'Allemagne le plus souvent au niveau du Land ou au niveau communal. 
Schéma 2 : Le système d'innovation national en Allemagne

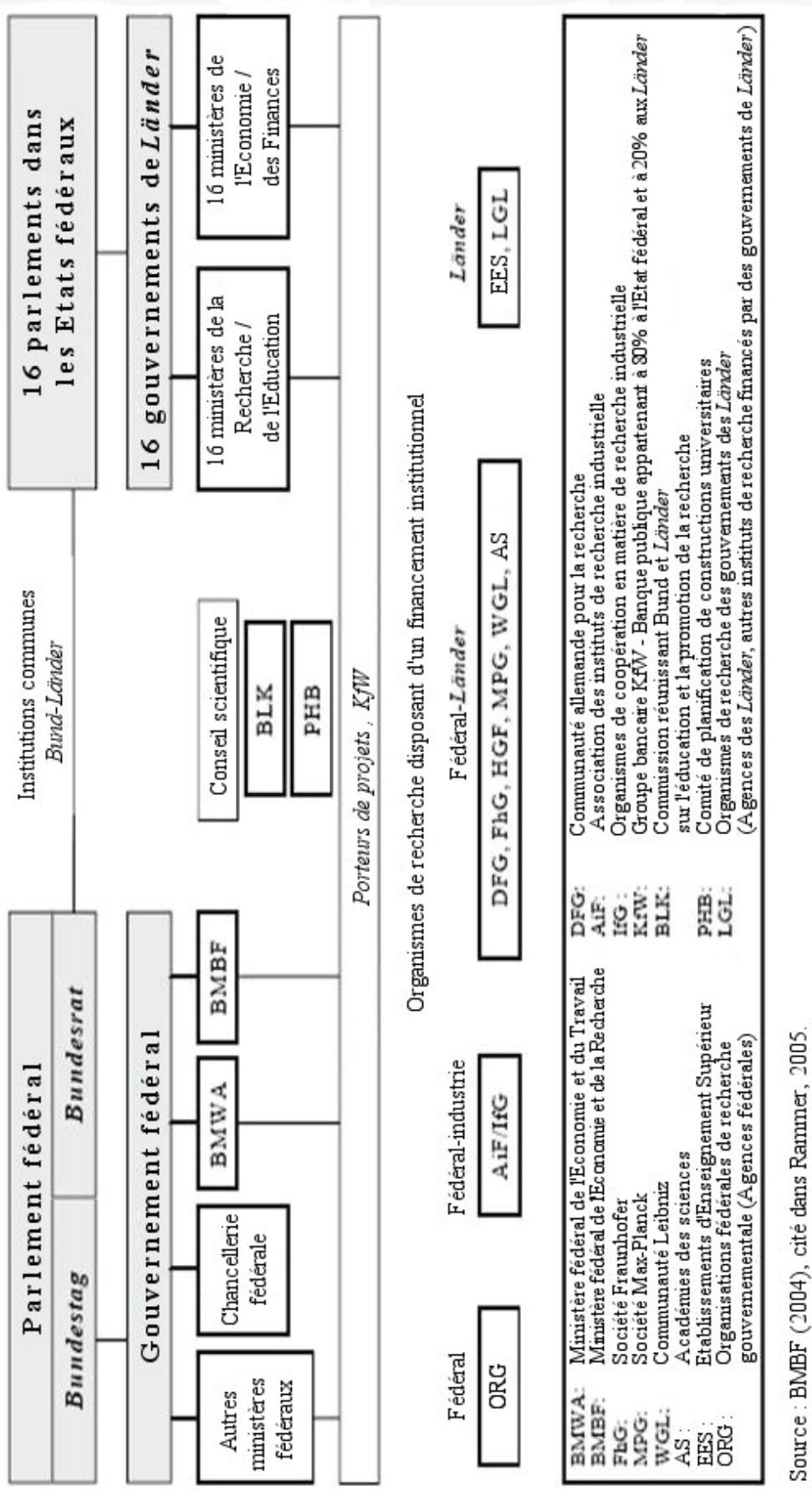


Dans ce cadre, les acteurs des réseaux d'innovation régionaux se recrutent parmi différents groupes (institutionnels), comme le montre le schéma 3 sur la participation des acteurs aux réseaux du programme InnoRegio (Kirschten, 2005).

\section{Schéma 3 : Acteurs d'un prototype de réseau d'innovation régional}

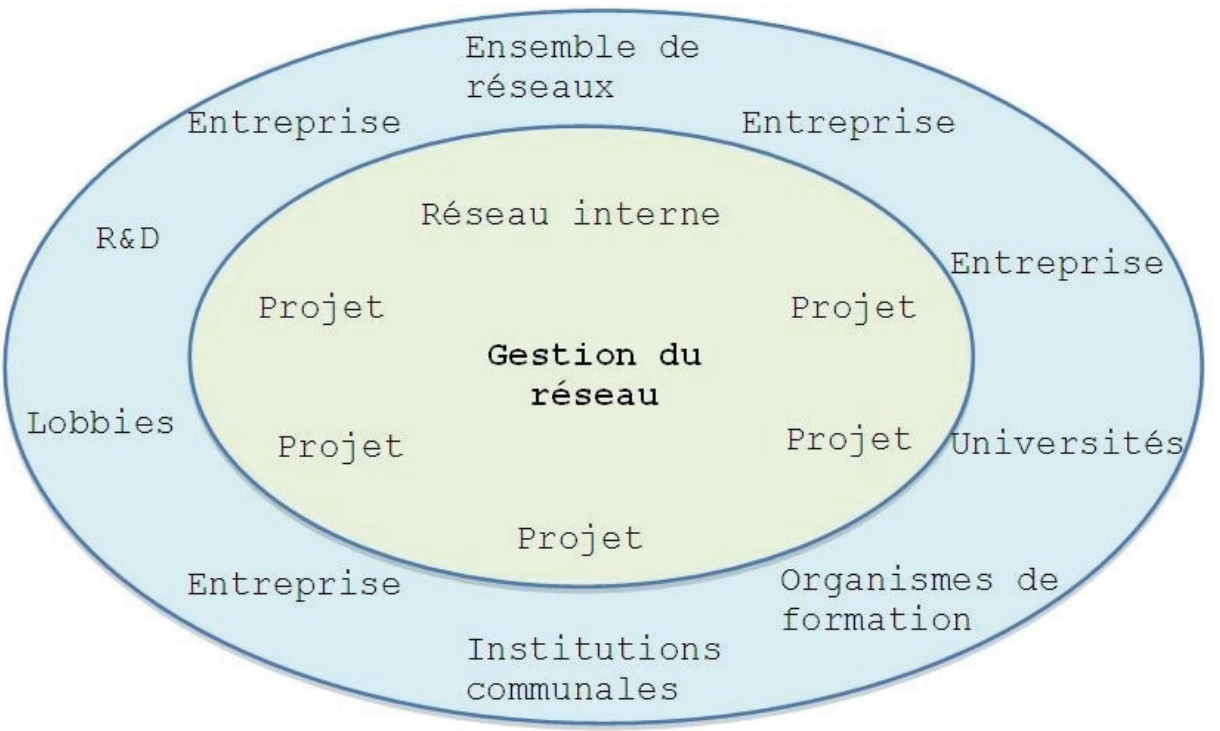

Source : Kirschten, 2005.

Les réseaux d'innovation régionaux sont généralement présentés de manière fortement positive, c'est-à-dire que leur création et leur existence sont considérées comme positives ; les avantages suivants sont mis en avant pour les acteurs et les régions: tout d'abord, on souligne les effets de synergie et d'apprentissage ainsi que l'accélération de l'apprentissage par la mise en réseau. Les compétences complémentaires présentes au sein du réseau doivent donner naissance, à moyen terme, à des compétences essentielles. Avec la combinaison potentielle de ressources du réseau, cela doit engendrer un potentiel supérieur permettant d'aborder des missions complexes. En outre, la/les capacité(s) (disponibilité de connaissances extérieures) doivent augmenter. On souligne également le partage des risques dans le réseau, ce qui entraîne une réduction des coûts et un gain de temps, et donc des avantages en termes d'efficacité et de compétitivité.

Souvent, les inconvénients potentiels des réseaux d'innovation ne sont pas autant mis en évidence; ils existent pourtant et ne doivent pas être négligés. Ainsi, la création de réseaux et leur entretien peuvent entraîner d'importants frais de coordination. De plus, l'étroitesse du réseau et de la coopération ainsi 
que le partage de travail peuvent créer des dépendances. Le « risque moral», c'est-à-dire une forme de déloyauté d'un ou plusieurs acteurs du réseau par rapport à celui-ci et aux autres acteurs, représente une autre source de problèmes avec les arrangements nuisibles au réseau ("shirking»). Les comportements de défection ou d'exploitation vis-à-vis des obligations de confidentialité ou des questions de droits de propriété intellectuelle entrent également dans cette catégorie de problèmes. De par leur coopération souvent très étroite, les acteurs accèdent mutuellement à des informations sensibles. Si ces informations sont utilisées aux dépens d'un acteur, sont divulguées ou font l'objet de violations de droits de propriété intellectuelle, cela réduit durablement l'efficacité et le succès des réseaux. Il peut également arriver que tous les acteurs ne profitent pas du travail en réseau de manière égale, ou que les partenaires accordent une importance différente à la réalisation des objectifs. (Becker et al., 2007 ; Asche et al., 2005).

\section{Les réseaux d'innovation régionaux : exemple de la Rhénanie du Nord-Westphalie}

Après avoir présenté la théorie, défini les réseaux d'innovation et la politique économique tournée vers l'innovation, voici une étude de cas portant sur trois réseaux d'innovation régionaux du Land de Rhénanie du Nord-Westphalie. Ces trois réseaux sont décrits et analysés sur les critères du contenu, des objectifs, de la structure, des partenaires et du financement; leurs implications pour le développement durable sont démontrées. Ces analyses reposent sur des recherches en ligne et bibliographiques ainsi que les observations des auteurs. Ont été choisis des réseaux dont la structure est marquée par le rôle dominant du monde politique et de l'enseignement supérieur. Cette analyse ne prétend pas être représentative. Pour cela, des efforts de recherche plus poussés seraient nécessaires. Afin de refléter la diversité des réseaux d'innovation régionaux existants, on a sélectionné des réseaux aux structures et aux contenus très différents.

Il existe en Rhénanie du Nord-Westphalie une multitude de réseaux dans lesquels l'enseignement supérieur, le monde politique et l'économie régionale coopèrent avec diverses fonctions et des contenus très variés. Il existe aussi des réseaux regroupant exclusivement des partenaires du monde économique ou des sociétés de développement. Par exemple, on peut citer les réseaux «Zukunftsinitiative Textil NRW» ou le regroupement des centres technologiques et de création d'entreprises «TGZ NRW ». De tels réseaux ne font pas l'objet de la présente analyse. On se concentrera ici sur des réseaux régionaux dans lesquels la politique a donné un élan initial pour encourager l'innovation et qui s'efforcent d'utiliser les résultats de la recherche universitaire. 


\subsection{Patente Gründungen Westfalen Ruhr (POWeR)}

\subsubsection{Contenu}

Le projet « Patente Gründungen Westfalen Ruhr» fait partie de l'offensive brevets Ruhr Westphalie (Patent Offensive Westfalen Ruhr, POWeR). Le programme POWeR a été fondé en 2002 à l'occasion du remaniement de la loi sur les inventions du personnel (Arbeitnehmererfindungsgesetz) ${ }^{6}$. Les universités de Bielefeld, Dortmund, Münster et Paderborn se sont regroupées dans le cadre de cette offensive pour former une alliance régionale. Celle-ci s'efforce, par des projets, des échanges d'expérience et d'information ainsi que des structures pluriuniversitaires, d'instaurer une culture durable de dépôt de brevets ainsi que la réalisation de processus de transfert innovants. Cela inclut la promotion de créations d'entreprises à partir de brevets, objectif du projet «Patente Gründungen » (cf. Université technique de Dortmund, 2006).

Depuis mars 2007, le projet est soutenu financièrement dans le cadre de l'initiative fédérale EXIST (Existenzgründungen aus der Wissenschaft, création d'activités indépendantes d'origine scientifique). Le projet réparti sur trois ans se compose des sous-projets suivants : (cf. Université technique de Dortmund, 2008) :

- le sous-projet «Sensibilisierung und Qualifizierung für Patente Gründungen » (Sensibilisation et qualification pour la création d'entreprise à partir de brevets) forme et qualifie des collaborateurs scientifiques dans des domaines pertinents du point de vue technologique ;

- le sous-projet «Ideen für Patente Gründungen » (Idées de création d'entreprise à partir de brevets) élabore des concepts organisés de création d'entreprise à partir d'ateliers d'idea mining ;

- le sous-projet «Verwaltungsworkshop Patente Gründungen » (Atelier de gestion des créations d'entreprise à partir de brevets) a pour objectif de sensibiliser l'administration des universités aux besoins spécifiques des inventeurs prêts à fonder une entreprise ;

- le sous-projet «Patente der regionalen Wirtschaft - Gründungen mit Hochschulen » (Brevets de l'économie régionale - créations d'entreprise avec l'enseignement supérieur) identifie des brevets «en sommeil » dans l'économie régionale de Westphalie, élabore pour ces brevets des concepts de création d'entreprise économiquement viables; avec ces concepts, des fondateurs, des entreprises et l'université, il crée de nouvelles entreprises et de nouveaux emplois pour la région ;

\footnotetext{
6 A l'occasion de ce remaniement, la notion de "privilège de professeur d'université » a été abolie. Désormais, les universités ont le droit de déposer des brevets sur les inventions de leurs employés et de les valoriser pour leur propre compte. Les professeurs d'université et le personnel scientifique ne disposent plus librement de leurs inventions.
} 
- le sous-projet «Profitcenter Patente Gründungen » (Création d'entreprise à partir de brevets, centre de profits) facilite, réalise et assure, en tant qu'incubateur d'entreprises, les idées de création du réseau POWeR ;

- le sous-projet « Handlungsleitfaden Patente Gründungen » (Guide de la création d'entreprise à partir de brevets) documente et publie les résultats du projet dans son ensemble.

\subsubsection{Structure}

Le projet « Patente Gründungen Westfalen Ruhr » est étalé sur trois ans, dans le cadre du partenariat POWeR. Ce regroupement est organisé sous forme de réseau sans structure juridique propre comme par exemple celle d'une association ou d'une société à responsabilité limitée (SARL). Il n'existe ni cotisation de membre, ni capital de départ commun. Le réseau POWeR et le projet «Patente Gründungen Westfalen Ruhr» sont coordonnés par l'unité de transfert de connaissances de l'Université technique de Dortmund. La collaboration s'effectue sur la base de réunions régulières de projet ou de réseau, d'accords de coopération et d'une stratégie commune de valorisation. Le projet s'engage entre autres :

- «à être un réseau régional pour la valorisation des résultats propres de recherche et développement ;

- à renforcer les créations d'entreprises et la coopération entre l'économie et la science dans le champ de valorisation de l'enseignement supérieur ;

- à intégrer dans son travail d'autres partenaires de réseau venant du milieu scientifique extra-universitaire, de l'économie régionale et des communes. ${ }^{7} »$

Les partenaires clés du réseau et du projet sont quatre universités. La gestion du réseau est prise en charge par l'unité de transfert de connaissances de l'Université technique de Dortmund. En outre, il existe dans le cadre de l'organisation des sous-projets des partenaires de coopération, par exemple pour le traitement et la valorisation des inventions, les conseils à la création, les conférences, l'animation d'ateliers ou le pilotage de la coopération avec les entreprises (cf. schéma 4). La coopération est en règle générale organisée sous forme d'accords de coopération ou de travail. Les sous-projets sont sous la responsabilité autonome des différents partenaires. Il existe également des promoteurs qui soutiennent le projet « Patente Gründungen Westfalen Ruhr». Ceux-ci ont pour mission, par leur notoriété et leurs contacts personnels, de soutenir la mise en œuvre durable du projet et de s'impliquer dans cette thématique lors de réunions de travail régulières. Les promoteurs sont notamment des dirigeants

\footnotetext{
${ }^{7}$ Proposition de projet du consortium d'universités POWeR pour le programme de subventions EXIST III, Dortmund, juillet 2006, page 1 .
} 
d'entreprises régionales de développement économique, de petites et moyennes entreprises ou de sociétés de participation. Le cercle élargi du réseau comprend par exemple les bailleurs de fonds, les porteurs de projet ainsi que les ministères responsables de la valorisation des brevets au niveau du Land et de l'Etat fédéral. Les cibles du projet sont les étudiants, les chercheurs, les administrations des universités ainsi que les entreprises de la région Ruhr Westphalie (cf. Université technique de Dortmund, 2006).

Schéma 4 : Structure du projet Patente Gründungen Westfalen Ruhr

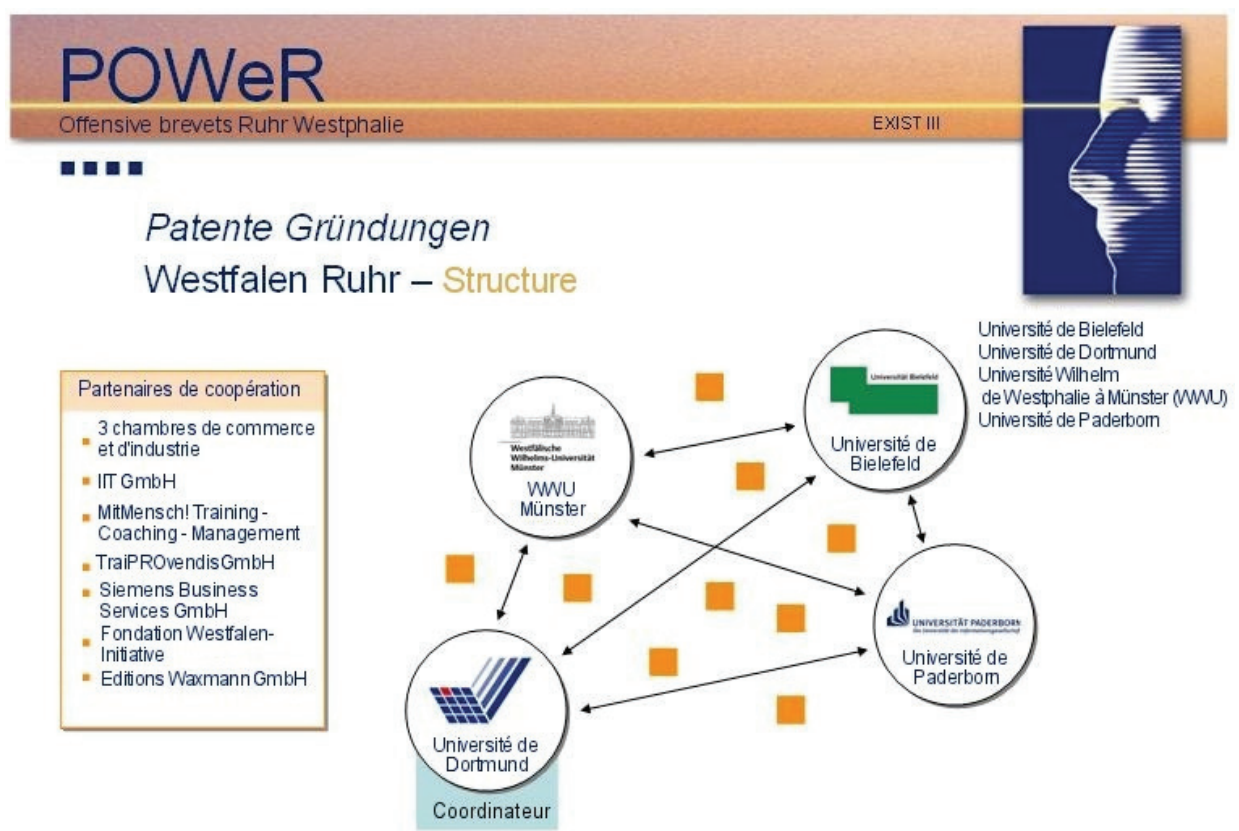

Source : cf. Université technique de Dortmund, 2007.

\subsubsection{Partenaires}

Les fonds sont apportés par le ministère fédéral de l'Economie et de la Technologie (Bundesministerium für Wirtschaft und Technologie, BMWI) et le Fonds social européen (FSE) qui financent le projet «Patente Gründungen Westfalen Ruhr » dans le cadre de l'initiative EXIST (Initiative Existenzgründungen aus der Wissenschaft) visant à promouvoir la création d'entreprise dans les milieux scientifiques. EXIST encourage une culture d'indépendance des universités et des instituts de recherche et, depuis 1998, a soutenu financière- 
ment plus de 30 réseaux en leur apportant plus de 45 millions $€$ (cf. FraunhoferInstitut, 2006).

Les partenaires principaux sont l'Université technique de Dortmund, l'Université Wilhelm de Westphalie à Münster, l'Université de Paderborn et l'Université de Bielefeld. Les partenaires de projet sont les Chambres de commerce et d'industrie de Nord-Westphalie, de Westphalie orientale à Bielefeld et à Dortmund, les sociétés Institut für Innovationstransfer an der Universität Bielefeld GmbH et MitMensch Training-Coaching-Management à Paderborn, la société de valorisation de brevets Provendis $\mathrm{GmbH}$, la société Siemens Business Services $\mathrm{GmbH} \& \mathrm{Co}$. OHG, la fondation Westfalen-Initiative ainsi que les éditions Waxmann Verlag (cf. schéma 4).

\subsubsection{Financement}

Le projet de réseau «Patente Gründungen Westfalen Ruhr» dispose d'un volume de subventions de 1,2 million $€$ sur trois ans (mars 2007 à mars 2010), qui servent à financer six sous-projets. Les coûts se répartissent entre frais de personnel et de matériel, moyens pour la conclusion de marchés et frais de déplacement. Chaque université partenaire est responsable de sous-projets et du budget prévu à cet effet. La subvention permet de proposer gratuitement les manifestations et les offres de conseils aux destinataires étudiants, administrateurs et chercheurs (cf. Université technique de Dortmund, 2006).

\subsubsection{Durabilité}

$\mathrm{Au}$ moment de cette contribution, le réseau avait notamment organisé dans le cadre de ce projet deux ateliers de gestion pour les collaborateurs des quatre universités partenaires, cinq manifestations pour le personnel scientifique avec au total 75 participants et créé une base de données de brevets avec les coordonnées et les informations sur les brevets de 5000 entreprises moyennes de la région.

Le projet inclut des objectifs qui doivent être mesurés régulièrement. Il n'est pas possible de procéder à une évaluation finale du projet, puisqu'il court encore jusqu'en mars 2010.

En raison de la structure du projet et des résultats obtenus jusqu'à présent, on peut envisager les alternatives suivantes pour un financement durable et une poursuite du projet sans aides publiques de l'Etat fédéral :

- reprise du personnel par les universités ou cofinancement avec les partenaires du projet ;

- mise en place d'offres payantes de formation initiale et continue pour les étudiants, les chercheurs et les administrations des universités ;

- élaboration de projets de continuation; 
- recherche de sponsors, fondations ou autres partenaires financiers extérieurs ;

- création d'une société de droit privé.

\subsection{Create.NRW}

\subsubsection{Contenu}

«Create.NRW » est un concours conçu par le ministère de l'Economie, du Mittelstand et de l'Energie du Land de Rhénanie du Nord-Westphalie.

Il s'agit d'un concours d'idées et de concepts visant à promouvoir l'économie culturelle et artistique en Rhénanie du Nord-Westphalie. Il s'adresse aux acteurs et aux entreprises du secteur créatif - l'industrie musicale, les éditeurs et libraires, le marché de l'art, le théâtre, le cinéma et la télévision, le design et la mode, la publicité, l'industrie des logiciels et des jeux ainsi que l'architecture - qui sont des générateurs d'impulsions et un moteur d'innovation dans leur branche respective, mais aussi dans toute l'économie et la société (ministère de l'Innovation, de la Science, de la Recherche et de la Technologie du Land de Rhénanie du Nord-Westphalie, MIWFT, 2007a).

L'objectif de ce concours est d'améliorer la force d'innovation et la compétitivité des petites et moyennes entreprises de Rhénanie du NordWestphalie, de créer et de sécuriser l'emploi. Il recherche et soutient des coopérations et des réseaux sortant de l'ordinaire entre entreprises, secteurs, universités, sociétés de promotion économique et acteurs de la scène locale.

Ce concours doit contribuer à :

- faire ressortir l'importance de l'économie de la culture et de la création en tant que facteur économique et innovant en Rhénanie du Nord-Westphalie ;

- affiner la compréhension de l'économie spécifique des secteurs créatifs ;

- générer des idées et des stratégies pour développer de nouveaux systèmes d'innovation régionaux ;

- renforcer les réseaux et clusters existants et nouveaux ;

- améliorer la compétence des indépendants et des petites entreprises de l'économie culturelle et artistique en matière d'entreprise et de marché ;

- créer des plates-formes, des marchés et des infrastructures susceptibles de servir de points d'ancrage à l'économie créative ;

- favoriser le transfert de savoir-faire entre les universités et les entreprises (MIWFT, 2007a). 


\subsubsection{Structure}

«Create.NRW » fait partie de l'offensive d'innovation du gouvernement du Land de Rhénanie du Nord-Westphalie qui cherche depuis 2007 à détecter et à soutenir les projets innovants d'excellence au moyen d'un total de 17 concours. ${ }^{8}$ Ceux-ci s'adressent aux acteurs de l'économie et de la science et visent avant tout les universités, les établissements de recherche et les petites et moyennes entreprises de Rhénanie du Nord-Westphalie. Les programmes des concours sont gérés par le ministère de l'Economie, du Mittelstand et de l'Energie du Land de Rhénanie du Nord-Westphalie et intègrent des structures supplémentaires de gestion et de contrôle (cf. schéma 5).

\section{Schéma 5 : Structures du programme NRW Ziel2}

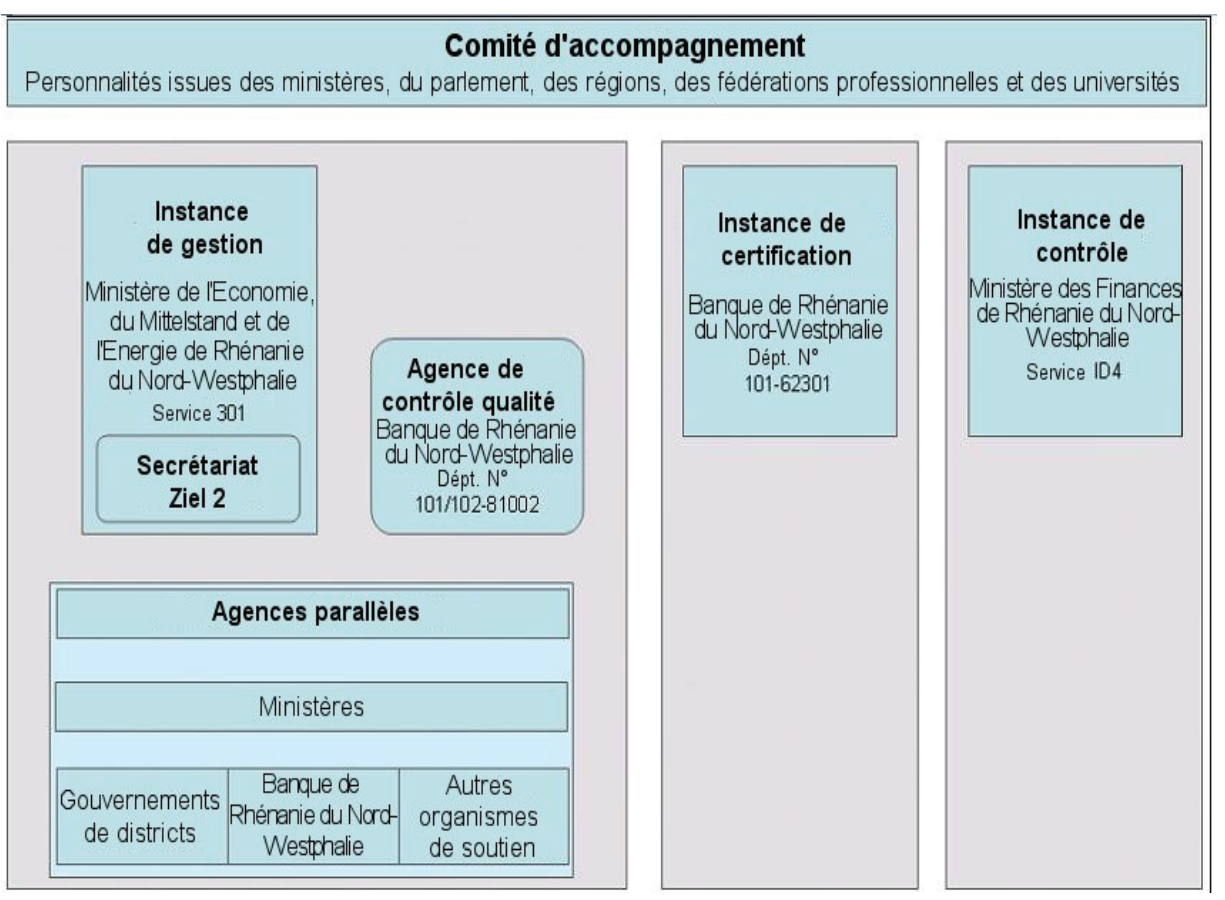

Source: Guide «Förderhandbuch Operationelles Programm (EFRE) 2007 - 2013 für das Ziel „Regionale Wettbewerbsfähigkeit und Beschäftigung“ für Nordrhein-Westfalen ».

Le concours d'idées et de concepts "Create.NRW » se décompose en deux phases : lors de la première phase, il était possible jusqu'à fin octobre 2007 de soumettre des idées de projets réussis dans l'économie culturelle et artistique.

\footnotetext{
${ }^{8}$ Ces concours font partie de la politique de clusters du Fonds européen de développement régional (FEDER). L'objectif de cette politique est de créer un environnement favorable à l'innovation et ainsi d'augmenter la compétitivité de l'économie du Land de Rhénanie du Nord-Westphalie
} 
Un jury a choisi les six idées qui lui semblaient les plus prometteuses et les a récompensées de $10000 €$ chacune. Lors de la seconde phase, il était possible jusqu'à fin mai 2008 de soumettre des concepts détaillés. La participation à la phase 2 était possible indépendamment de la phase 1. Parmi les dossiers, 10 projets pilotes ont été sélectionnés mi-2008. Après la sélection, un processus officiel de soumission et d'autorisation se déroule jusqu'à fin août 2008. Le concours se tient en 2008 et en 2009 ; les projets présentés doivent être terminés au plus tard fin 2011 (cf. MIWFT, 2008).

\subsubsection{Partenaires}

L'autorité de gestion du réseau est le ministère de l'Economie, du Mittelstand et de l'Energie (MWME) du Land de Rhénanie du Nord-Westphalie. Le concours est financé par le Fonds de développement régional de l'Union européenne (FEDER). La société Gesellschaft für innovative Beschäftigungsförderung GIB $G m b H$ de Bottrop s'intercale comme organe de coordination. Les demandeurs et partenaires du projet peuvent être des universités, des établissements de recherche, des acteurs de l'économie culturelle et artistique - par exemple des associations ou des organismes de droit public - ainsi que des petites et moyennes entreprises.

Les entreprises candidates peuvent employer jusqu'à 250 personnes et générer un chiffre d'affaires annuel atteignant 50 millions $€$; elles doivent être indépendantes des grandes entreprises. Il n'y aucune disposition relative au nombre de partenaires (cf. MIWFT, 2007a ; 2007b).

\subsubsection{Financement}

Pour les deux éditions du concours Create.NRW (2008/2009), il est prévu un budget global à hauteur de 15 millions $€$. Chaque édition doit soutenir 10 projets pilotes, ce qui donne une subvention moyenne de $750000 €$. Au maximum $80 \%$ des dépenses éligibles sont prises en charge, tandis que les candidats doivent en financer au minimum $20 \%$ par leurs propres moyens ou avec l'aide de partenaires extérieurs (cf. MIWFT, 2007a).

Seules des dépenses sont subventionnées, pas des coûts. Ainsi, ne sont reconnues comme dépenses admissibles que celles qui sont causées directement par le projet. Suivant ce principe, les frais de personnel ne peuvent en règle générale être pris en compte que s'ils concernent du personnel supplémentaire nécessaire à la réalisation du projet. Les dépenses liées au personnel existant ne peuvent être subventionnées qu'à titre exceptionnel en cas d'exigences particulières. Le paiement des allocations octroyées est soumis au principe de remboursement des dépenses. Un paiement n'est donc possible que si le destinataire a déjà procédé aux dépenses éligibles et les justifie au moyen de factures acquittées ou autres pièces comptables similaires. Il en résulte que le 
destinataire des subventions doit préfinancer les dépenses du projet (MIWFT, 2007b).

\subsubsection{Durabilité}

Dans la première phase du concours, 117 concepts ont été présentés (cf. MIWFT, 2007b). Parmi les dossiers, 10 projets pilotes ont été sélectionnés mi2008. Après la sélection, une procédure officielle de demande et d'autorisation a lieu jusqu'à fin août 2008. Les résultats des projets sélectionnés ne peuvent pas encore être évalués, puisqu'ils commencent seulement courant 2008.

Si l'objectif d'augmenter la compétitivité du Land de Rhénanie du NordWestphalie dans le domaine de l'économie culturelle et artistique était atteint, il existerait différentes possibilités de poursuite des projets après écoulement de la période de subvention :

- poursuite du concours avec des moyens du Fonds européen de développement régional (FEDER) si c'est possible et encore nécessaire ;

- reprise du personnel financé par les projets par les candidats et/ou les partenaires de projet ;

- élaboration d'offres payantes de manifestations, conseils, formation initiale et continue pour les groupes cibles des projets ;

- intégration des résultats des projets dans des structures existantes des candidats et/ou partenaires des projets ;

- recherche de sponsors privés, donateurs ou autres partenaires financiers extérieurs pour poursuivre les projets.

\subsection{InnovationsAllianz NRW}

\subsubsection{Contenu}

Selon ses propres indications, le groupement InnovationsAllianz NRW est la plus grande alliance d'universités d'Allemagne pour le transfert de connaissances et de technologies. Il a démarré en janvier 2007. Dans le cadre de ce réseau partenarial, les universités et les Fachhochschulen veulent contribuer à l'extension des capacités d'innovation du Land. D'ici 2015, la Rhénanie du Nord-Westphalie sera le premier Land du pays en termes d'innovation. Pour cela, le Land et l'économie doivent investir plus dans la recherche et le développement. Le groupement InnovationsAllianz a pour objectif de renforcer les transferts de connaissances et de technologies des universités, par exemple au travers d'actions de relations publiques et de projets conjoints ou sur contrats avec l'industrie. Un autre de ses points forts 
est le développement et la mise en place de normes communes de qualité, par exemple dans la promotion de la création d'entreprises, la valorisation des brevets ou le développement de modèles de coopération avec l'industrie (cf. Innovationsallianz e.V., 2007).

InnovationsAllianz soutient et promeut ces objectifs au moyen d'une offre de prestations de services adaptées à la demande et par un travail actif de relations publiques et de publicité. Au centre de cette offre se trouve l'échange d'expériences et de découvertes coordonné et animé par des professionnels ainsi qu'un travail commun engagé sur les thèmes pertinents pour le transfert (Innovationsallianz e.V., 2007). Grâce à un site Internet, les intéressés à l'intérieur et à l'extérieur des universités peuvent rechercher des interlocuteurs dans les universités en remplissant un formulaire. On y trouve également les actualités sur les universités et les manifestations du groupement. ${ }^{9}$

\subsubsection{Structure}

Les organes d'InnovationsAllianz e.V. sont le bureau et l'assemblée des membres. Les organismes consultatifs sont le conseil consultatif et le conseil d'administration. La mission de l'assemblée des membres est notamment d'adopter un plan d'activité et de donner quitus, élire ou révoquer les membres du bureau. Le bureau est composé de représentants de l'enseignement supérieur ainsi que des sociétés de transfert des universités concernées. Quatre tâches lui incombent :

- communication et coordination des travaux transversaux ;

- établissement et optimisation, en particulier des structures de valorisation de droit privé des universités ;

- lobbying et promotion de la recherche, création d'entreprises, valorisation de brevets ;

- label, marketing, publicité, plate-forme de communication.

Les missions du conseil consultatif consistent à conseiller le bureau et à apporter un soutien durable à InnovationsAllianz. Y siègent notamment des représentants du gouvernement du Land, de la banque de financement NRW.Bank, des médias ainsi que des chambres de commerce et d'industrie.

Quant au conseil d'administration, il assiste le bureau dans l'aménagement et l'organisation des prestations de service d'InnovationsAllianz. Y siègent des représentants du secteur opérationnel du transfert ainsi que des entreprises privées de valorisation dans lesquelles les universités ont des parts (cf. Innovationsallianz e.V., 2006 ; schéma 6).

\footnotetext{
${ }^{9}$ Les nouvelles sont principalement des communiqués de presse universitaires, des manifestations organisées par les établissements d'enseignement supérieur et InnovationsAllianz, des études et la publication de projets de soutien à l'échelle du Land (cf. http://www.innovationsallianz.nrw.de [consulté le 06/09/10]).
} 


\section{Schéma 6 : Structure du réseau InnovationsAllianz}

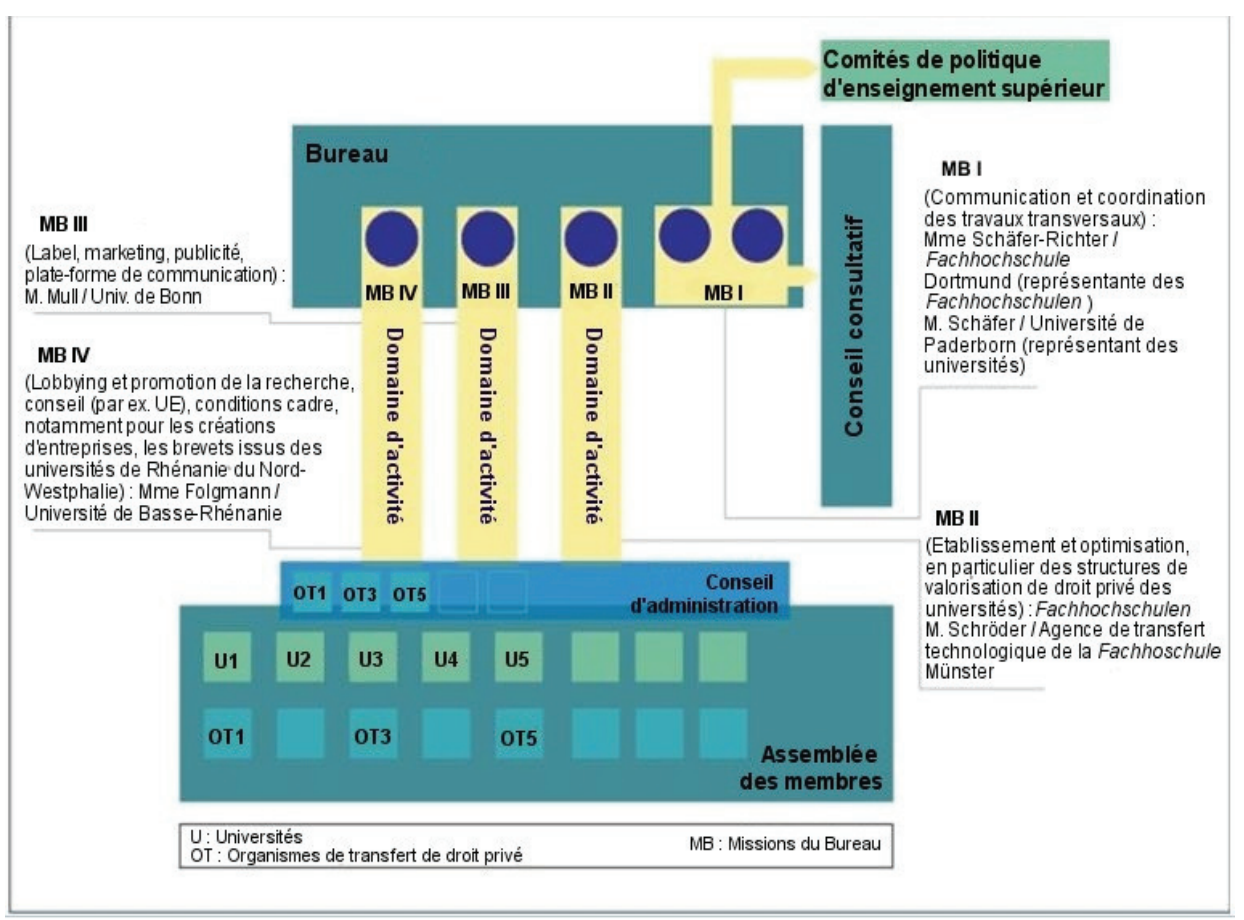

Source : http://www.innovationsallianz.nrw.de/organisation/organisationsinfos.html [consulté en janvier 2009].

\subsubsection{Partenaires}

Le ministère de l'Innovation, des Sciences, de la Recherche et de la Technologie (MIWFT) de Rhénanie du Nord-Westphalie ainsi que 23 universités financent le réseau InnovationsAllianz. Les établissements participants sont $:^{10}$

\begin{tabular}{|l|l|}
\hline $\begin{array}{l}\text { Fachhochschule Aachen (Aix-la- } \\
\text { Chapelle) }\end{array}$ & Fachhochschule Gelsenkirchen \\
\hline RWTH Aachen (Aix-la-Chapelle) & Fachhochschule Köln (Cologne) \\
\hline Fachhochschule Bielefeld & $\begin{array}{l}\text { Rheinische Fachhochschule Köln } \\
\text { (Cologne) }\end{array}$ \\
\hline Université de Bielefeld & $\begin{array}{l}\text { Katholische Fachhochschule } \\
\text { Nordrhein-Westfalen }\end{array}$ \\
\hline Fachhochschule Bochum & Fachhochschule Lippe und Höxter \\
\hline $\begin{array}{l}\text { Evangelische Fachhochschule } \\
\text { Rheinland-Westfalen-Lippe/Bochum }\end{array}$ & $\begin{array}{l}\text { Fachhochschule Niederrhein } \\
\text { (Krefeld / Mönchengladbach) }\end{array}$ \\
\hline
\end{tabular}

\footnotetext{
${ }^{10}$ Plus d'informations sur les membres d'Innovationsallianz :

cf. http://innovationsallianz.nrw.de/innovationsallianz/im-profil/mitglieder.html [consulté le 06/09/10]
} 


\begin{tabular}{|l|l|}
\hline Université de Bonn & Fachhochschule Münster \\
\hline Fachhochschule Bonn-Rhein-Sieg & Université de Münster \\
\hline Fachhochschule Dortmund & Université de Paderborn \\
\hline Université technique de Dortmund & Université de Siegen \\
\hline Universität Duisburg-Essen & $\begin{array}{l}\text { Fachhochschule Südwestfalen } \\
\text { (Sud-Westphalie) }\end{array}$ \\
\hline Fachhochschule Düsseldorf & \\
\hline
\end{tabular}

\subsubsection{Financement}

Le ministère de l'Innovation, des Sciences, de la Recherche et de la Technologie (MIWFT) de Rhénanie du Nord-Westphalie soutient le réseau InnovationsAllianz par un financement annuel de $160000 €$. Les 23 universités participantes ont apporté $120000 €$ supplémentaires via leurs cotisations et leurs apports en personnel et en matériel (cf. Innovationsallianz e.V., 2007). Le bureau perçoit une indemnité de représentation.

Le montant des cotisations et des apports de financement est consacré à l'élaboration et à la mise en œuvre d'offres conformes au but de l'association. Les autres détails sont réglés par des statuts et un règlement intérieur. Aucune offre payante n'est incluse. La dissolution de l'association ne peut être obtenue que si neuf dixièmes des membres le décident (cf. Innovationsallianz e.V., 2006).

\subsubsection{Durabilité}

A l'heure de cette intervention, il n'est pas possible d'évaluer les résultats du travail de réseau d'Innovationsallianz. Jusqu'à présent, le site Internet du réseau annonçait notamment les manifestations du réseau et des universités participantes, les nouvelles des universités et des informations sur les programmes de subvention actuels. En mai 2008, une manifestation d'information de l'alliance s'est tenue à Bruxelles sous le titre «Scientific Entrepreneurship - Unternehmerische Wissenschaft in Nordrhein-Westfalen » (Entreprenariat scientifique - science d'entreprise en Rhénanie du NordWestphalie), dans le cadre de laquelle des réseaux de création d'entreprises des universités du Land se sont présentés aux décideurs européens (cf. Innovationsallianz e.V., 2008). Aucun résultat officiel n'a encore été fourni en ce qui concerne le nombre de demandes de conseils transmises via le formulaire de contact électronique.

Pour mesurer le succès du réseau InnovationsAllianz, il faut définir des critères de réussite clairs, et ce, principalement dans l'optique d'une poursuite des financements publics par le Land de Rhénanie du Nord-Westphalie. De par la structure d'Innovationsallianz e.V., on peut en principe envisager les 
alternatives suivantes pour pérenniser le travail de l'association si les membres le souhaitent :

- augmentation de la cotisation d'adhésion ou entrée de nouveaux membres ;

- poursuite avec l'aide de partenaires financiers et sponsors extérieurs ;

- élaboration d'offres payantes de manifestations, conseils, formation initiale et continue sur les thèmes du transfert de connaissances et de technologies ;

- développement de projets de transfert;

- intégration des résultats du projet dans des structures existantes des partenaires et des membres.

L'IDÉE ET L'INTENTION DE BASE DES RÉSEAUX d'innovation régionaux sont d'exploiter plus efficacement le potentiel des régions, voire de compenser des situations de départ défavorables par un soutien politique et/ou un coup de pouce financier. Cependant, il est indispensable de se demander si les spécificités caractéristiques d'une "région» offrent réellement un point de départ prometteur pour une croissance endogène entraînée par l'innovation. Les établissements de recherche et de formation ou les services de R\&D localisés des entreprises peuvent stimuler l'activité économique régionale. Cependant, il n'entre pas forcément dans les intentions (de l'ensemble) des institutions et surtout des entreprises d'être le moteur du développement régional. L'intervention publique doit donc être mise en œuvre de manière bien réfléchie et soigneusement dosée pour être utile à l'économie et rentable.

\section{Traduction de Marie-Céline GEORG}

\section{Indications bibliographiques}

Asche M., BAuHus W., KAdDATZ B., SEEL B., Verwertungsnetzwerke: Eine Perspektive für den Technologietransfer, Waxmann, Münster, 2005

Becker T., DAmmer I., HowaldT J., Killich S., LoOSE A., Netzwerkmanagement: Mit Kooperation zum Unternehmenserfolg, Springer Verlag, Berlin/Heidelberg, 2007

BELITZ H., DEUTSChES INSTITUT FÜR WIRTSCHAFTSFORSCHUNG (Diw), «Forschung und Entwicklung in multinationalen Unternehmen», Studien zum deutschen Innovationssystem, n8, janvier 2004, p. 1-55

BÖHME G., STEHR N. (ed), The Knowledge Society, D. Reidel Publishing Company, Dordrecht, 1986

BUNDESMINISTERIUM FÜR BILDUNG UND FORSCHUNG (BMBF), Starke Impulse für die nationale Technologieentwicklung: Bioregionen in Deutschland, BMBF, Bonn, 2003

BURGER-MENZEL B., Fachhochschulen in regionalen Innovationsnetzwerken - das Beispiel FH Brandenburg. Intervention tenue lors de la conférence finale Coronas Metropolitanas, Potsdam, 30 novembre 2006

CAMAgni R., «Local 'Milieu', Uncertainty and Innovation Networks: Towards a New Dynamic Theoriy of economic Space», in CAMAGNi R., Innovation Networks: Spatial Perpectives, Belhaven Press, Londres, 1991, p. $122-157$ 
ETZKOwITZ H., MiT and the Rise of Entrepreneurial Science, Routledge, Londres/New York, 2002

EtzKowitz H., LeYdesdorfF L. (ed), Universities and the Global Knowledge Economy. A Triple Helix of University-Industry-Government Relations, Continuum International Publishing Group, Londres, 1997

Etzkowitz H., Webster A., Healey P., Capitalizing knowledge: new intersections of industry and academia, State University of New York Press, Albany, 1998

FLORIDA R., «Toward the Learning Region», Futures, vol. 25, n 5, juin 1995, p. 534-553

FRAUNHOFER-INSTITUT FÜR SYSTEM- UND INNOVATIONSFORSCHUNG, EXIST - Existenzgründungen aus Hochschulen, Bericht der wissenschaftlichen Begleitung zum Förderzeitraum 1998-2005 (Kurzfassung), Fraunhofer-Institut für System- und Innovationsforschung, Karlsruhe, 2006

FRITSCH M., Marktdynamik und Innovation, Duncker \& Humblot, Berlin, 2004

Fritsch M., Henning T., Slatchev V., Steigenberger N., Hochschulen, Innovation, Region, Sigma, Berlin, 2007

Granovetter M., «Economic Action and Social Structure: The Problem of Embeddedness», American Journal of Sociology, vol. .91, $\mathrm{n}^{\circ} 3$, novembre 1985, p. 481-510

HARITZ A., Innovationsnetzwerke - Ein systemorientierter Ansatz, DUV/Gabler, Wiesbaden, 2000

HEIDENREICH M., «Regionale Netzwerke in der globalen Wissensgesellschaft», in WEYER J., ABEL J. (ed), Soziale Netzwerke: Konzepte und Methoden der sozialwissenschaftichen Netzwerkforschung, Oldenbourg, Munich, 2000, p. [n.c.]

HeIDENREICH M., «Knowledge-Based Work: An International Comparison», International Management, vol. $8, \mathrm{n}^{\circ} 3$, printemps 2004, p. $87-110$

InNOvationsAllianZ E.V., Satzung des Vereins InnovationsAllianz der NRW-Hochschulen e.V., InnovationsAllianz e.V., Düsseldorf, 2006

INNOVATIONSALLIANZ E.V., InnovationsAllianz gegründet - NRW-Hochschulen stellen Wissenstransfer auf eine neue Basis [en ligne] InnovationsAllianz e.V., Dusseldorf, 2007 : http://www.innovationsallianz.nrw.de [consulté le 07/01/10]

INNOVATIONSALLIANZ E.V., InnovationsAllianz in der NRW-Landesvertretung in Brüssel, [en ligne] InnovationsAllianz e.V., Dusseldorf, 2008 : http://www.innovationsallianz.nrw.de [consulté le 07/01/10]

KIRSCHTEN U., Risiken der Zusammenarbeit in Innovationsnetzwerken konzeptionelle Überlegungen und empirische Ergebnisse, Intervention tenue à l'université d'Erfurt lors de la septième journée professionnelle «Technologie- und Innovationsmanagement» (TIM) du 27 au 29 octobre 2005

KOSCHATZKY K., «Regionale Entwicklungskonzepte zur Initiierung wirtschaftlichen Wachstums», in PleschaK F (ed), Wachstum und Innovation. Strategien, Probleme und Erfahrungen FuE-intensiver Unternehmen, DUV/Gabler, Wiesbaden, 2003, p. [n.c.]

KOWOL U., KROHN W., «Innovationsnetzwerke. Ein Modell der Technikgenese», in BeCHMANN G., HALFMANN J., RAMMERT W. (ed), Jahrbuch Technik und Gesellschaft 8, Campus, Francfort/New York, 1995, p. $77-105$

KOwOL U., KROHN W., «Innovation und Vernetzung. Die Konzeption der Innovationsnetzwerke», in WEYER J., ABEL J. (ed), Soziale Netzwerke: Konzepte und Methoden der sozialwissenschaftlichen Netzwerkforschung, Oldenbourg, Munich, 2000, p. 135-160

Marshall A., Principles of Economics, MacMillan, Londres, 1966

MinisteriUM FÜR WIRTSCHAFt, MitTELSTAND Und ENERGIE (MIWFT), Create.NRW, Gesucht: Die besten Ideen für die Kreativ- und Kulturwirtschaft, Wettbewerbsaufruf, MIWFT, Dusseldorf, 2007a

MINISTERIUM FÜR WIRTSCHAFT, MITTELSTAND UND ENERGIE (MIWFT), Großes Interesse am Landeswettbewerb Create.NRW, Ministerin Thoben: NRW will Marktplatz für die Kreativen sein, Pressemitteilung, MIWFT, Dusseldorf, 2007b 
MINISTERIUM FÜR WIRTSCHAFT, MitTELSTAND UND ENERGIE (MIWFT), Create.NRW, Förderwettbewerb 2008, FAQs - Fragen und Antworten zum Wettbewerb, MIWFT, Dusseldorf, 2008

Mowery D. C., Nelson R. R. (ed), Sources of Leadership. Studies of Seven Industries, Cambridge University Press, Cambridge/New York, 1999

OCDE, Managing National Innovation Systems, OCDE, Paris, 1999

OCDE, «Bio Information», OECD Observer, n² 240/241, Paris, décembre 2003, p. [n.c.]

PETER V., Institutionen im Innovationsprozess. Eine Analyse anhand der biotechnologischen Innovationssysteme in Deutschland und Japan, Physica-Verlag, Heidelberg, 2003

PIORE M. J., SABEL C. F, The second industrial divide, Basic Books, New York, 1984

Porter M. E., On Competition - Cluster and Competition: New Agendas for Companies, Governments, and Institutions, Harvard Business Review Book, Cambridge MA, 1998

RAMMER C., ZENTRUM FÜR EUROPÄISCHE WIRTSCHAFTSFORSCHUNG (ZEW), «Unternehmensdynamik in Deutschland 1995-2003: die Rolle forschungs- und wissensintensiver Branchen und eine Einordnung im internationalen Vergleich.», Studien zum deutschen Innovationssystem, n ${ }^{\circ} 1-2005$, nomvembre 2004, p. 1-35

RAMMERT W., «Zwei Paradoxien einer innovationsorientierten Wissenspolitik: Die Verknüpfung heterogenen und die Verwertung impliziten Wissens», Soziale Welt, vol. 54, n 4, octobre 2003, p. 483-508

RÜTTGERS J., Regierungserklärung vor dem Landtag Nordrhein-Westfalen am Mittwoch, 13. Juli 2005, [s.e.], [s.1.], 2005

SCHUMPETER J. A., Capitalism, socialism and democracy, Harper and Brothers, New York, 1942

SOSKICE D., «Technologiepolitik, Innovation und nationale Institutionengefüge in Deutschland», in NASChOld F., SOSKICE D., HANCKÉ B., JÜRGENS U. (ed), Ökonomische Leistungsfähigkeit und institutionelle Innovation, Sigma, Berlin, 1997, p. 319-348

SpIElKamP A., CZARnitzKi D., RAMMER C., «Ideenmotor Hochschule? Wege des Wissenstransfers und die Bedeutung von Spin-offs», in HeINZE R. G., Schulte F. (ed), Unternehmensgründungen zwischen Inszenierung, Anspruch und Realität, Westdeutscher Verlag, Wiesbaden, 2002, p. 79-100

STAHL T., SchreIBER R., Regionale Netzwerke als Innovationsquelle. Das Konzept der ,Lernenden Region' in Europa, Campus Verlag, Francfort/New York, 2003

STEHR N., Arbeit, Eigentum und Wissen: Zur Theorie von Wissensgesellschaften, Suhrkamp, Francfort, 1994

STIFTERVERBAND FÜR DIE DEUTSCHE WISSENSCHAFT, Innovationsfaktor Kooperation: Bericht des Stifterverbandes zur Zusammenarbeit zwischen Hochschulen und Unternehmen, edition Stifterverband, Essen, 2007

Technische Universität Dortmund, Transferstelle, Ideenskizze Patente Gründungen Westfalen Ruhr, Projektvorschlag des POWeR-Hochschulkonsortiums für das Förderprogramm EXIST III, Technische Universität Dortmund, Dortmund, 2006

Technische Universität Dortmund, Transferstelle, Powerpoint-Folien Patente Gründungen Westfalen Ruhr, Technische Universität Dortmund, Dortmund, 2007

Technische Universität Dortmund, Transferstelle, Projektstruktur Patente Gründungen Westfalen $R u h r$ [en ligne] : www.patente-gruendungen.de, Lien : "Die Projekte“, [consulté le 07/01/10]

TEILJOHANN V., «Die italienische Debatte um Industriedistrikte. Das Beispiel der Emilia-Romagna», in KRUMBEIN W (ed), Ökonomische und politische Netzwerke in der Region. Beiträge aus der internationalen Debatte, LIT Verlag, Münster/Hamburg, 1994, p. [n.c.]

WeINGART P., Die Stunde der Wahrheit? Zum Verhältnis der Wissenschaft zu Politik, Wirtschaft und Medien in der Wissensgesellschaft, Velbrück Wissenschaft, Weilerswist, 2001. 



\title{
Le pôle biotech de Munich : une « culture de l'innovation »
}

\author{
Horst DOMDEY
}

Dans le secteur des biotechnologies, l'Allemagne occupe toujours le premier rang européen : en 2006, le pays comptait près de 500 entreprises dans ce secteur, regroupées le plus souvent au sein de pôles de compétitivité. En première position de ceux-ci figure la BioRegio de Munich construite autour de la localité de Martinsried, quatrième cluster biotechnologique européen après Cambridge, Copenhague/Lund et Zurich/Bâle, et dont le succès repose en grande partie sur le développement de nouvelles molécules. En effet, les deux seuls médicaments issus des biotechnologies allemandes actuellement présents sur le marché ont été conçus par l'entreprise MediGene, implantée sur le site de Martinsried.

A l'heure où on s'interroge en France sur les orientations à donner à la politique des pôles de compétitivité et où un nouveau partage des responsabilités entre l'Etat et les acteurs de la recherche est envisagé, l'approche bottom-up privilégiée outre-Rhin est susceptible d'apporter de nouveaux éclairages sur le rôle de l'initiative privée comme facteur clé de succès dans le développement des clusters.

Le Professeur Horst Domdey, l'un des fondateurs de la société MediGene, président depuis 1998 de la structure de coordination du pôle de Martinsried (BioM), ainsi que du Centre de promotion de l'innovation et de la création d'entreprises (Innovations- und Gründerzentrum Biotechnologie, IZB), évoque dans cette contribution la naissance et l'organisation de la BioRegio de Munich.

\section{Genèse du cluster}

Le pôle de biotechnologies de Munich n'est pas né ex nihilo, bien qu'il ait vu le jour voici trente ans dans un petit village au sud-ouest de Munich. La proximité de la capitale du Land de Bavière avec ses deux universités et ses centres de recherche, l'attractivité naturelle du site ainsi que la présence de personnel qualifié ont contribué au choix de Martinsried dans l'implantation en 1972 d'un nouvel Institut Max-Planck de biochimie, ce qui a, soit dit en passant, brutalement mis fin à la ruralité de Martinsried. Cet Institut Max-Planck est né du regroupement de toute une série d'instituts, transférés là pour être mis en réseau. $\mathrm{Au}$ fil du temps ont été ensuite intégrés à ce nouvel Institut diverses équipes de l'Université de Munich, dont le Centre d'études génomiques, fondé en 1984. Je suis arrivé pour ma part en 1976 à l'Institut Max-Planck de biochimie de Martinsried afin d'y préparer ma thèse de doctorat, avant de poursuivre un temps mes recherches à Lausanne et aux Etats-Unis.

Le cluster s'est donc développé autour de l'Institut Max-Planck de biochimie, dans un environnement où les premières entreprises de biotechnologies commençaient à voir le jour, comme Mikrogen. Cette entreprise a été fondée à 
Martinsried en 1989 par deux chercheurs de l'un des centres de recherche en microbiologie de l'Université Ludwig Maximilian de Munich. Créée par spin-off, elle développe et commercialise des méthodes de diagnostic médical. Elle compte actuellement près de cent collaborateurs et réalise un chiffre d'affaires annuel supérieur à 10 millions $€$. Il est intéressant de relever que cette entreprise a pu être créée sans apport de capital-risque ou de capital extérieur et qu'elle appartient toujours à ses deux fondateurs. Suite à cela sont apparues, de 1992 à 1994, les premières entreprises faisant appel au capital-risque, communément nommées les « $3 \mathrm{M} »$ : MorphoSys, Micromet et MediGene. J'ai personnellement pris part à la création de MediGene dont le siège a tout naturellement été installé à Martinsried, dans cet environnement porteur en termes de $\mathrm{R} \& \mathrm{D}$ de biologie moléculaire. La spirale était enclenchée...

\section{MediGene AG}

La création en 1994 de l'entreprise de biotechnologies MediGene AG s'inscrit dans le prolongement de l'activité de recherche du Centre d'études génomiques de Munich. Employant près de 175 collaborateurs, la société est aujourd'hui également implantée en Angleterre, à Oxford et aux Etats-Unis, à San Diego. Cotée à la bourse de Francfort depuis 2000, elle se consacre à la recherche, au développement et à la commercialisation de nouveaux médicaments pour le traitement de cancers, de maladies de peau et de maladies auto-immunes. Première entreprise allemande de biotechnologies, MediGene laissait jusqu'à présent à des entreprises partenaires le soin de distribuer ses produits. Elle a pour ambition de se lancer en 2008 dans la commercialisation de ses deux médicaments déjà présents sur le marché, Eligard $\AA$ et Polyphenon ${ }^{\circledR}$ E-Salbe. Un troisième, Oracea $\AA$, fait actuellement l'objet d'une demande d'autorisation de mise sur le marché. Malgré un CA de 23,9 millions $€$ en 2007, l'entreprise enregistre toujours des pertes (-29,9 millions $€$ ) liées en grande partie aux coûts élevés de R\&D, qu'elle espère compenser en 2008 par une forte croissance de CA. (SH)

\section{Entrée tardive des pouvoirs publics dans le jeu des acteurs locaux}

C'est donc la création d'un réseau par des chercheurs, élargi ensuite à des banquiers et des entrepreneurs, qui a mené à la naissance, puis à la croissance progressive du cluster. Le ministère bavarois de l'Economie n'a décidé que par la suite, en 1995, de mettre en place un Centre de promotion de l'innovation et de la création d'entreprises: 1'Innovations- und Gründerzentrum Biotechnologie $(I Z B)$. J'ai pris part aux discussions qui ont été menées à l'époque : ce centre pouvait-il apporter de nouvelles perspectives, quelle devait en être la surface ? On a voulu être prudent en fixant dans un premier temps l'étendue des locaux à $800 \mathrm{~m}^{2}$ seulement. Depuis, cet espace a été élargi pour atteindre plus de 15000 $\mathrm{m}^{2}$. Indépendamment de cela, en 1996, les biotechnologies ont connu une impulsion particulière en Allemagne : du fait des moyens financiers limités dont disposait le ministère fédéral de la Recherche, il a été décidé de mettre en place un concours dans le cadre duquel les régions allemandes pouvaient présenter leur candidature, et ce en vue de devenir une "région modèle » dans le domaine des biotechnologies modernes. Nous avons alors pris part à ce concours BioRegio et, à notre plus grande joie, l'agglomération de Munich a fait partie des trois gagnantes parmi les dix-sept régions participantes. Les deux autres lauréats étaient la région de Heidelberg, c'est-à-dire le triangle Rhin-Neckar comprenant 
les villes de Mannheim, Ludwigshafen et Darmstadt, ainsi que la région de la Rhénanie avec les Universités de Düsseldorf, de Cologne et d'Aix-la-Chapelle.

Nous nous attendions alors à des félicitations du ministre de la Recherche et une poignée de mains en signe de reconnaissance. Or à notre grande surprise, le gouvernement fédéral nous a annoncé que, dans les régions sélectionnées, la recherche au sein des jeunes entreprises de biotechnologies recevrait un soutien financier de la part du gouvernement fédéral, la priorité dans l'allocation des moyens du ministère fédéral de la Recherche étant accordée aux trois régions gagnantes [en 1996, le financement s'élevait à 25 millions $€$ pour chacun des trois clusters retenus ; $\mathrm{SH}]$. Mais ce qui a été au moins tout aussi important que ce soutien financier, c'est que les régions ont été invitées à s'auto-organiser. Il fallait créer les conditions afin de faire émerger les meilleurs concepts. Les BioRegios devaient acquérir la compétence nécessaire pour prendre des décisions de manière autonome. Quant aux start-up en leur sein, elles ont été elles aussi soumises à concurrence, les aides étant attribuées en priorité à celles présentant les concepts les plus porteurs. La répartition de ces moyens financiers limités s'est donc faite sur la base d'une compétition à la fois entre régions et, au niveau de chacune d'entre elles, entre start-up.

\section{Mise en place de Bio ${ }^{M}$, structure de coordination du pôle}

Nous avons alors décidé très rapidement qu'il nous fallait mettre en place un mode d'organisation spécifique pour coordonner nos activités de manière professionnelle. Ainsi, vingt-cinq ans après la fondation de l'Institut Max-Planck de biochimie de Martinsried, nous avons créé, en 1997, une plateforme pour mieux fédérer les acteurs de R\&D et du marché. Nous avons donné à cette société non pas la forme alors habituelle d'une SARL, mais celle d'une SA dans l'espoir d'obtenir des moyens financiers plus conséquents ; ce choix s'est révélé pertinent par la suite. Il est vrai que le contexte était porteur : la politique d'innovation du gouvernement fédéral, de même que celle des Länder, comportait alors également un volet visant le développement d'un financement des start-up technologiques par le marché. Cette SA, la $\mathrm{Bio}^{M} A G$, avait pour mission de conseiller, d'informer, d'encourager la mise en place de partenariats, de promouvoir le site économique, d'organiser des manifestations et de mener des activités de lobbying. Mais il s'agissait aussi, et c'était ce qu'il y avait de particulier dans l'organisation du réseau, de proposer un apport de capital d'amorçage aux entreprises. Plus tard, en 2002, nous y avons même intégré un petit fonds de capital-risque.

Ce choix que nous avons fait de proposer des financements en termes de capital d'amorçage ou de capital-risque afin de dynamiser la création d'entreprises issues du monde de la recherche nous différencie d'autres organisations. Nous avons dans notre portefeuille des entreprises dans lesquelles nous avons investi 3,2 millions $€$; nous avons pu lever en outre 200 millions $€$ de capital-risque. Le Land de Bavière y a trouvé également son intérêt car une partie de 
cet argent lui est revenu sous la forme d'impôts. Nous représentons ainsi une source de revenus aussi bien pour la République fédérale que pour le Land.

\section{Bio $^{m}$, une structure réticulaire évolutive}

$B i o^{M}$ est une tête de réseau, l'émanation d'un réseau qui s'est constitué grâce à l'initiative privée. Nous avions un domaine d'innovation, un nouveau cluster qui s'est créé de lui-même. Il n'a pas été mis sur pied de manière artificielle mais il était là, et il a bénéficié ensuite de la mise en place d'une organisation pour le gérer. Cela signifie que les partenaires de ce réseau étaient déjà présents. Il ne restait plus qu'à les relier entre eux. Tout l'art de la gestion du réseau consiste à reconnaître très tôt les nouvelles tendances et à y adapter les services proposés. Par exemple, au début, il a beaucoup été discuté des moyens de financement et des possibilités d'entrée en bourse. Plus tard, quand les entreprises ont atteint une certaine taille, s'est posée la question du développement de médicaments, des accords nécessaires en vue de la réalisation de tests et des autorisations de mise sur le marché. Nous avons ainsi construit un réseau multidimensionnel avec des activités multidimensionnelles qui apparaissent puis s'estompent selon les périodes.

\section{Les acteurs du réseau de biotechnologies de Munich/Martinsried}

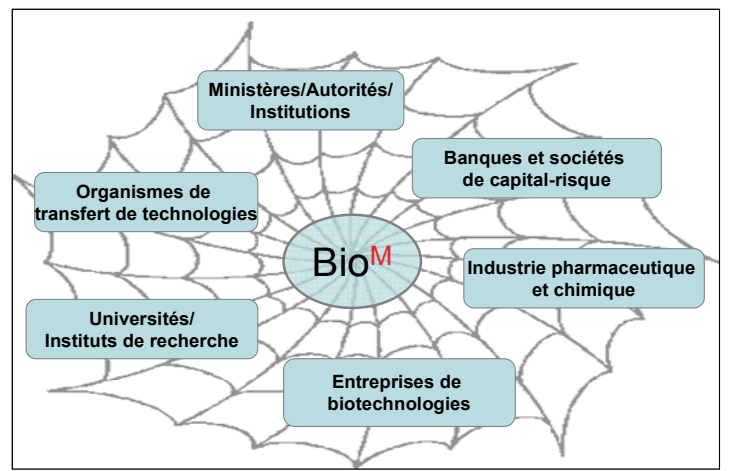

Source : Domdey, 2008

La $B i o^{M} A G$ a été scindée en deux à l'automne 2006, lorsqu'un autre domaine d'activité est venu s'ajouter : la gestion du cluster des biotechnologies à l'échelle de l'ensemble de la Bavière. Depuis, les fonctions de gestion du réseau sont exercées par la nouvelle Bio ${ }^{M} \mathrm{GmbH}$ Biotech Cluster Development, qui est donc non seulement une structure de coordination, mais aussi une sorte de guichet unique pour toute personne œuvrant dans le marché des biotechnologies. La $B i o^{M} A G$ Munich Biotech Development, quant à elle, se concentre exclusivement sur le capital-risque, dont l'amorçage et le coaching. Ces entités, dont les équipes travaillent en étroite collaboration, offrent une complémentarité idéale. 


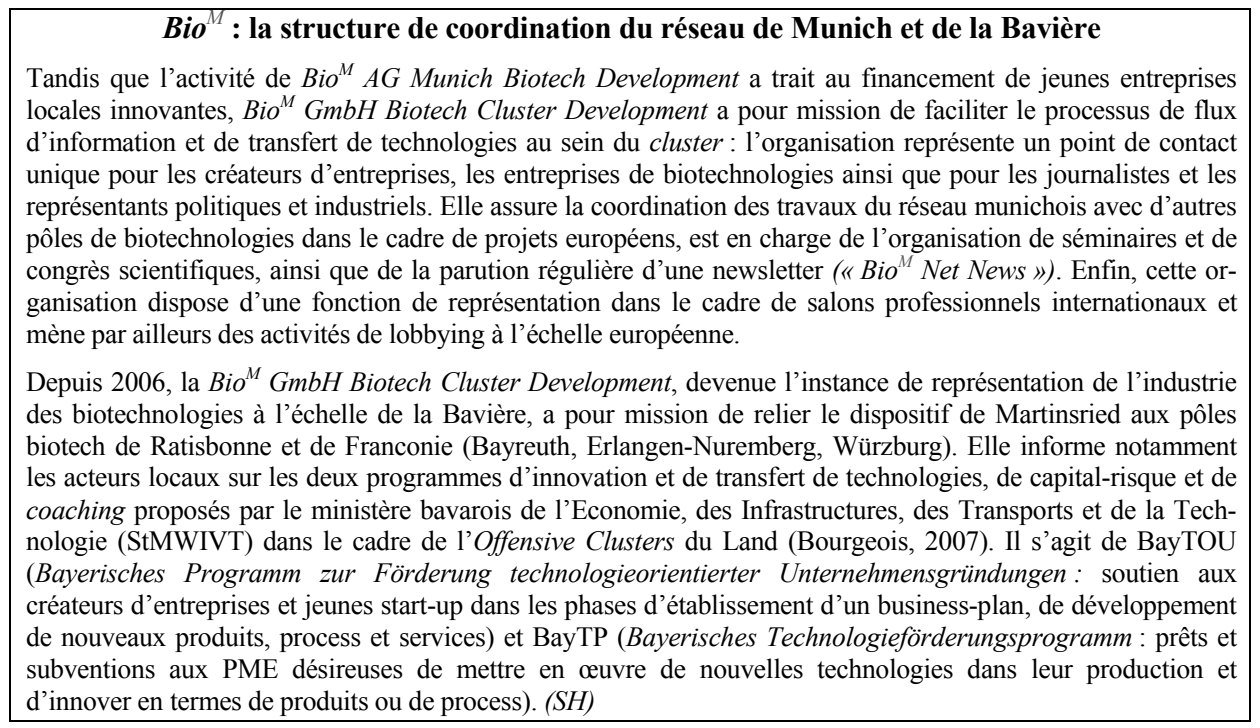

\section{Communication et activités de réseau, y compris à l'échelle européenne}

Nous soignons la communication, en organisant des manifestations, ou en tentant d'apparaître au moins une fois par semaine dans la presse locale, ainsi qu'une fois par mois dans les quotidiens suprarégionaux, ou dans les autres médias comme la télévision. Nous organisons également des journées portes ouvertes au sein des entreprises de biotechnologies de Martinsried ou de Munich, nous recevons des groupes de visiteurs et leur présentons le cluster. Mais nous menons aussi un intense lobbying. Nous sommes ainsi à l'origine de la création de la fédération d'entreprises Biotechnologie-Industrie-Organisation Deutschland (BIO Deutschland). En 2005, j'ai lancé un groupe de travail regroupant les pôles régionaux allemands de biotechnologies, nommé Arbeitskreis der BioRegionen Deutschlands (AK BRD), dont l'objectif était d'aboutir à une coopération entre des régions concurrentes à l'échelle fédérale. Sur la base de ce groupe de travail, nous sommes arrivés à la constitution d'un Conseil des régions européennes de biotechnologies (Council of European Biotech Regions, CEBR), dont fait partie par exemple le groupement d'intérêt public Genopole situé à Evry. Enfin, nous disposons également de groupes de travail plus spécialisés. C'est ainsi que nous essayons d'avoir une certaine influence politique. Mais c'est malheureusement plus difficile que nous ne le pensions.

\section{La mise en réseau des pôles biotech de Bavière}

Nous avons commencé à intensifier la mise en réseau des sites bavarois de Würzburg, Ratisbonne, Bayreuth... Et nous avons identifié certains problèmes. En premier lieu, malgré le potentiel considérable que représente l'exploitation des biotechnologies blanches (ou biotechnologies industrielles), il reste beaucoup à 
faire dans ce domaine. Nous avons donc créé un sous-cluster «Biotechnologies blanches » pour lequel nous avons pu rassembler 5 millions $€$ de financement de l'Etat fédéral, 5 millions $€$ à l'échelle du Land et 15 millions $€$ de plus du côté de l'industrie. Par ailleurs, nous souhaitons développer la réalisation d'études cliniques en Bavière. Pour cela, nous avons repéré les centres qui procèdent à ce genre d'études en vue de mettre en place des processus de coopération étroite.

\section{Facteurs clés : le tissu local d'activités économiques ou scientifiques...}

Certains facteurs locaux sont indispensables à la constitution d'une culture de l'innovation. Pour notre cluster, cela commence effectivement par les établissements de recherche. A Munich, nous avons la chance d'avoir deux universités de pointe qui, avec l'Université de Karlsruhe dans le Land de Bade-Wurtemberg, ont été distinguées "Universités d'Elite» à l'issue du premier tour du concours d'excellence du gouvernement fédéral en octobre 2006. Pourquoi l'Université Ludwig Maximilian et l'Université Technique de Munich sont-elles si performantes ? Parce qu'elles sont en situation de concurrence permanente. Par ailleurs, elles sont dotées d'un CHU. Nous disposons en outre de deux Instituts d'enseignement supérieur spécialisés dans les villes de Munich et de Weihenstephan, de trois Instituts Max-Planck (de neurobiologie, de biochimie et de psychiatrie) et du Centre Helmholtz de recherche sur l'environnement et la santé. Cela constitue la masse critique nécessaire à l'innovation et explique le taux de $95 \%$ de créations d'entreprises émanant du domaine scientifique. A l'opposé, si vous regardez d'autres sites européens à la pointe dans les biotechnologies, par exemple ceux de Copenhague/Lund ou de Zurich/Bâle, vous verrez que les nouvelles entreprises sont en grande part issues de l'industrie, ce qui est compréhensible : les deux groupes pharmaceutiques Roche et Novartis sont implantés à Bâle.

\section{... et une culture favorable au transfert de technologies}

A l'origine d'un tel cluster, il y a donc toujours un environnement porteur, et une culture favorable au transfert de technologies. Outre la présence d'un tissu scientifique dense et de plateformes de transfert, de bonnes conditions cadre valorisent cette culture de l'innovation. Par exemple pour ce qui est de l'offre en surfaces de bureaux ou de la rapidité avec laquelle la mise en place d'un laboratoire de recherche en génétique peut recevoir l'accord des autorités, et des autorités qui travaillent réellement en collaboration avec les entreprises.

Dans la région de Munich, nous disposons des deux premières plateformes de transfert de technologies d'Allemagne, qui sont totalement indépendantes des universités. Il s'agit de Max Planck Innovation pour les Instituts Max-Planck et d'Ascenion pour les Instituts Helmholtz. S'ajoutent à cela les plateformes de transfert de technologies des universités. Mais celles-ci, qui n'existent que depuis 2002 et qui ont connu des débuts difficiles, en partie en raison d'un sousfinancement, sont encore en cours de développement. Par ailleurs, le Centre de 
promotion de l'innovation et de la création d'entreprises, actuellement présent sur deux sites, contribue activement au transfert de technologies. C'est la seule structure à la fondation de laquelle le Land de Bavière a grandement participé. On pense souvent que le cluster de biotechnologies de Munich a pu se constituer grâce à l'octroi de subventions. Or c'est faux. Les deux Centres de promotion de l'innovation et de la création d'entreprises, qui ont peut-être coûté dans l'ensemble 60 millions $€$, n'ont pas été financés uniquement par des moyens alloués par le Land de Bavière, mais une grande partie de leur financement provient de prêts accordés par des banques privées, actuellement en cours de remboursement.

\section{Le cluster de biotechnologies de Munich}

Le quatrième cluster européen s'est nettement développé ces dix dernières années : il comprend actuellement non moins de 191 entreprises dans le domaine des sciences de la vie, qui emploient près de 20000 personnes. Il s'agit d'entreprises pharmaceutiques, de filiales de grands groupes, de CRO (Contract Research Organisations) et de PME de biotechnologies, qui comptent à elles seules pour plus de la moitié des entreprises du réseau et occupent 2500 collaborateurs.

En l'espace de dix ans, la région biotech a connu un essor considérable non seulement grâce au soutien financier du ministère fédéral de la Recherche qui s'élève à 45 millions $€(25$ millions $€$ ont été accordés à l'issue du concours BioRegio, puis près de 20 millions $€$ dans le cadre du programme BioChancePlus), mais aussi et surtout grâce aux 2 milliards $€$ de capitaux privés qui ont permis de financer l'industrie locale des biotechnologies. En résulte une forte croissance du nombre d'entreprises de biotechnologies : tandis qu'en 1997, le cluster comprenait 31 PME pour 370 collaborateurs, ce nombre avait plus que triplé en 2007, où 104 entreprises employaient 2500 personnes. Le chiffre d'affaires de ces entreprises a par ailleurs plus que doublé entre 2001 et 2007, passant de 125 à près de 300 millions $€$. Un léger bémol cependant en termes de dépenses de R\&D : malgré un pic en 2002 avec un investissement de 225 millions $€$, elles ont connu une légère baisse pour revenir à ce niveau en 2006.

Bien que le volume de financement des entreprises ait fortement augmenté en 2000 (cette augmentation s'explique notamment par deux entrées en bourse qui ont contribué à hauteur de 250 millions $€$ aux investissements, 250 millions $€$ supplémentaires ayant été injectés par des fonds de capital-risque privés cette même année), la région doit faire face à un apport de capitaux insuffisant, le volume de cet apport ayant fortement chuté jusqu'en 2003 pour ne remonter que légèrement depuis. Ainsi, alors que 500 millions $€$ ont été investis dans les biotechnologies en 2000, cette somme ne s'élève plus qu'à 278 millions $€$ en 2006 . Le manque de capitaux disponibles sur le marché européen, accompagné d'un trop grand nombre de créations d'entreprises fondées sur des business plans (encore) imprécis et la trop faible incitation pour les scientifiques à commercialiser les résultats de leur recherche constituent les défis que le cluster aura à relever dans les années à venir. (SH)

\section{Les compétences managériales des chercheurs sont déterminantes}

Les entrepreneurs représentent un facteur déterminant, car ce sont eux qui font avancer les choses. Les huit entreprises cotées en bourse depuis 1998 avec une capitalisation de 200 à 400 millions $€$ ont fait taire les critiques qui pensaient en 1994, année de la création de MediGene, que ce qui fonctionne aux Etats-Unis, à San Francisco ou à Boston, ne pourrait pas fonctionner en Allemagne, ni en Bavière au sein d'une économie naguère agricole, et encore moins dans un petit village comme Martinsried. La culture de l'innovation est largement présente en Allemagne aussi, mais comme partout, il faut, pour l'activer, des personnalités : des chercheurs et patrons capables de concevoir leur activité comme faisant partie intégrante d'une dynamique ouverte de réseau. Et il faut entre autres des chercheurs animés par l'esprit d'entreprise et dotés de compétences managériales. 


\section{Bio $^{M}$ : une mission d'aide à l'auto-assistance}

Notre mission au sein de $\mathrm{Bio}^{M}$ est de consolider ces multiples maillons d'un même réseau qui sont à la base de la constitution du cluster. Il ne faut en effet pas oublier l'existence de sous-réseaux. Par exemple, au sein des entreprises de biotechnologies, les responsables du développement clinique travaillent en réseau avec leurs homologues. Il en va de même pour les business developers ou les chefs d'entreprise. Il faut donc continuer de multiplier des liens transversaux pour mailler entre eux ces éléments de réseau verticaux et renforcer ainsi, au niveau horizontal, l'interconnexion de ces divers maillons. Ce n'est qu'alors que se constituera un réseau possédant une solidité et une stabilité comparables à celle d'une toile d'araignée. Notre organisation est pour ainsi dire l'araignée qui a généré cette toile, et notre approche est celle de l'aide à l'auto-assistance. Nous espérons que notre travail ne sera un jour plus nécessaire et que nous pourrons, à terme, disparaître. Le succès de $B i o^{M}$ sera en effet avéré lorsque personne n'aura plus besoin de nos services. C'est-à-dire quand, au sein des réseaux de pôles de biotechnologies allemands et européens, le cluster de Munich sera à même de générer sa propre croissance; en un mot : de mener la 'vie autonome' de tout pôle d'activités hautement compétitif.

Traduction de Solène HAZOUARD

\section{Indications bibliographiques}

BouRgeOIS I., «Bavière : la patiente construction d'une économie performante», in BOURGEOIS I. (dir.), Allemagne : compétitivité et dynamiques territoriales, CIRAC, Cergy-Pontoise, 2007, p. 77-94

BOURGEOIS I., «Le 'miracle biotechnologique' allemand», Regards sur l'Economie Allemande, n 57/2002, juillet 2002, p. 11-22

Guiot C., «L'essor des biotechnologies médicales en RFA», Regards sur l'Économie Allemande, n 77/2006, juillet 2006, p. 19-24

BAYERISCHES STAATSMINISTERIUM FÜR WIRTSCHAFT, INFRASTRUKTUR, VERKEHR UND TECHNOLOGIE, Offensive des clusters bavarois [en ligne] : http://www.cluster-bayern.de [consulté le 07/01/10]

Bio-Deutschland-GeschäfTSSTElle, Bio-Deutschland [en ligne] : www.biodeutschland.org [consulté le 07/01/2010]

BioM Biotech Cluster Development GMBH, BioM [en ligne] : www.bio-m.org [consulté le 07/01/2010]

BIOCOM PROJEKTMANAGEMENT GMBH, biotechnologie. de [en ligne] : www.biotechnologie.de [consulté le 07/01/2010]

MediGene AG, MediGene [en ligne] : www.medigene.de [consulté le 07/01/2010]. 


\title{
Le facteur humain au cœur du réseau bavarois Sensorik
}

\author{
Hubert STEIGERWALD
}

Avec $30 \%$ de parts de marché et un chiffre d'affaires global, tous acteurs confondus, de près de 30 milliards $€$ en 2007, l'Allemagne est aujourd'hui leader mondial dans le domaine des capteurs de mesure et de commande employés dans l'automobile, les sciences du vivant ou les technologies de l'environnement. L'activité se concentre dans le sud du pays, notamment dans le Land de Bavière qui rassemble près d'un quart des entreprises du secteur. Certaines font partie des 35 membres et 75 partenaires du réseau Sensorik, implanté dans le BioPark de Ratisbonne. Visant à stimuler les transferts de technologies, la création d'entreprises et la formation de personnel qualifié, le cluster labellisé "réseau de compétences ॥ (Kompetenznetz) par le ministère fédéral de l'Economie en 2007 figure parmi les lauréats du concours "Réseaux de compétences 2009 - Pour un développement pérenne des réseaux ॥. II a placé l'individu au cœur de sa stratégie. Ce choix s'est révélé judicieux, comme en témoignent les résultats encourageants des premières évaluations menées en 2008, après seulement deux années d'existence.

Dès lors, peut-on s'inspirer de cet exemple de réussite de ce côté-ci du Rhin, où 35 projets de plates-formes d'innovation, aux fonctions équivalentes à celles du réseau allemand, ont été présélectionnés par l'Etat et la Caisse des Dépôts le 5 février dernier dans le cadre de la politique française des pôles de compétitivité ? Dans sa contribution, Hubert Steigerwald, manager de Sensorik, également directeur du Partenariat Stratégique Sensorik et de la Sensorik-Bayern $\mathrm{GmbH}$, revient sur la genèse et les axes stratégiques de l'un des 2017 clusters recensés par l'Observatoire européen des clusters.

\section{Un partenariat stratégique s'est organisé autour d'acteurs préexistants...}

La région de Ratisbonne, située à proximité de Munich, se caractérise par une forte concentration d'entreprises dans le domaine des capteurs de mesure et d'asservissement (Sensorik). Le service d'expansion économique de la ville de Ratisbonne, ayant saisi le potentiel de ces entreprises en matière d'attractivité territoriale, avait initié dès juin 2003 un partenariat stratégique : à l'époque, 14 responsables économiques et académiques de la ville et du district se sont rassemblés dans une perspective de coopération. De nombreuses réunions se sont tenues, parfois en comité très restreint, jusqu'à ce que le cœur initial du réseau finisse par s'étendre : en août 2006, Sensorik comptait déjà 23 membres...

...puis a été institutionnalisé en 2007

Une nouvelle impulsion a été donnée en février 2006 par le ministère bavarois de l'Economie, des Infrastructures, des Transports et des Technologies (StMWIVT), 
qui a fait de notre secteur d'activité l'un des domaines technologiques clés de son programme de promotion des clusters. Notre partenariat stratégique s'est alors doté d'un statut juridique avec la création, le 11 mai 2006, d'une association à but non lucratif qui, dans les mois qui ont suivi, a endossé le rôle de plate-forme bavaroise dans notre domaine : la Strategische Partnerschaft Sensorik e.V. Cette formalisation juridique a permis de percevoir les fonds alloués par le ministère bavarois de l'Economie et d'enregistrer les cotisations des membres, dont le montant pouvait parfois atteindre $10000 €$. Puis en juillet 2007, nous avons décidé de constituer une SARL, la Sensorik-Bayern GmbH. Pour deux raisons: les acteurs économiques étaient de plus en plus nombreux à souhaiter que nous accomplissions des prestations de services, et le statut de SARL nous autorisait, contrairement à celui d'association, à réaliser des bénéfices. Le cluster s'est ainsi développé dans la continuité.

\section{L'Allemagne, leader mondial dans le domaine des capteurs}

Qu'ils possèdent des propriétés mécaniques, électriques, chimiques, biochimiques ou optiques, les capteurs sont intégrés à des systèmes de réglage et de commande. Agissant comme une véritable interface avec leur environnement, ils peuvent notamment contribuer à déterminer le taux de sucre dans le sang dans le cadre d'un traitement ciblé du diabète, à allier une utilisation réduite de carburant à une meilleure performance automobile, à améliorer la qualité des processus industriels et à en réduire le coût ou encore à localiser une explosion nucléaire à n'importe quel endroit de la planète. Il s'agit par conséquent d'une technologie clé aux interconnexions multiples : technique automobile, sciences du vivant, technologies de l'environnement, automatisation, mécatronique...

En 2007, le secteur des capteurs de mesure et d'asservissement a réalisé 22 milliards $€$ de chiffre d'affaires outreRhin. Affichant une croissance annuelle de $6,5 \%$ à $12,5 \%$ sur la période $2004-2007$, il consacre non moins de $40 \%$ de sa production à l'export et se compose essentiellement de PME : plus de la moitié des entreprises du secteur emploient moins de 50 personnes, $83 \%$ moins de 250 . Le pays dénombre 900 producteurs de capteurs de réglage et de commande. Si on inclut les prestataires de services dédiés, les bureaux d'étude ainsi que les agents commerciaux indépendants, ce chiffre s'élève à près de 2000 , pour un total de 230000 à 250000 collaborateurs. L'Allemagne est leader mondial du secteur avec $30 \%$ de parts de marché. La Bavière y occupe le premier rang en termes de CA et d'implantation, rassemblant sur son territoire près d'un quart des entreprises. A l'échelle fédérale, les intérêts de la branche sont représentés par la fédération AMA (Arbeitsgemeinschaft Messwertaufnehmer e.V.) : fondée en 1980, elle compte aujourd'hui près de 450 membres, parmi lesquels 70 centres de recherche. Elle publie régulièrement des statistiques de marché, s'implique dans la formation continue et organise des manifestations comme le salon annuel Sensor + Test de Nuremberg (www.ama-sensorik.de [consulté le 06/09/10]). (SH)

\section{La Cluster-Offensive du ministère bavarois de l'Economie}

Dans le cadre de cette politique, le ministère bavarois de l'Economie accorde à 19 clusters une aide de 50 millions $€$ au total répartie sur cinq ans (2006-2011), Sensorik s'étant vu attribuer pour sa part 1,2 million $€$. Le Land souhaite ainsi renforcer l'attractivité du site économique de la Bavière, où les grandes entreprises industrielles ont bien souvent fait le choix de délocaliser. Par conséquent, l'initiative vise plus spécifiquement les PME, plus fortement ancrées à l'échelle locale et régionale. A travers le soutien à des domaines technologiques clés, elle a pour vocation de stimuler l'innovation, condition sine qua non du maintien et du développement de l'emploi dans la région.

Ce programme incite à la création de plates-formes de coordination de l'activité des réseaux, dont le pilotage stratégique est assuré par un porte-parole, 
tandis que la gestion opérationnelle est aux mains d'un manager et de son équipe. Chaque manager détermine pour son cluster des objectifs, parmi lesquels celui de l'autonomie financière car les moyens attribués dans le cadre de la ClusterOffensive, constituant au départ entre $80 \%$ et $100 \%$ du capital, diminuent progressivement pour se réduire à terme à $35 \%$. Pour notre part, nous avons pour ambition d'accéder à l'autonomie en l'espace de trois ans, via la rétribution de nos prestations de services et l'obtention de financements lors de manifestations.

\begin{tabular}{|l|}
\hline Allianz Bayern Innovativ : l'Alliance pour l'Innovation en Bavière \\
Avec l'Alliance pour l'Innovation, le gouvernement de Bavière s'est doté d'un instrument visant à stimuler la \\
compétitivité territoriale du Land. Il s'agit dans un premier temps d'y développer, via la Cluster-Offensive, les \\
réseaux de coopération entre universités, centres de recherche, entreprises, prestataires de services et capital- \\
risqueurs dans 19 secteurs clés et/ou domaines de hautes technologies, selon trois objectifs : transposer plus \\
rapidement les résultats de la recherche académique dans le monde de l'entreprise sous la forme de nouveaux \\
produits ou procédés, intensifier la coopération entre les entreprises et leurs fournisseurs locaux tout au long de \\
la chaîne de valeur et enfin consolider l'attachement des entreprises au site économique bavarois. En partenariat \\
avec le réseau dédié à l'électronique de puissance (Cluster Leistungselektronik), Sensorik s'inscrit dans l'un des \\
19 thèmes d'avenir de ce programme de promotion des clusters. Outre un soutien ciblé par secteur d'activité, \\
l'Alliance pour l'Innovation a pour vocation d'encourager et de renforcer des potentiels de croissance \\
endogènes dans chaque région constitutive du Land. (SH)
\end{tabular}

\section{Assurer le leadership de la Bavière dans le domaine des capteurs}

Fort de mon expérience passée dans les activités opérationnelles des groupes Siemens et EADS, j'ai insisté sur la nécessité de fixer des objectifs précis lors de la candidature de Sensorik au programme du ministère bavarois de l'Economie. Où nous situons-nous actuellement? Où en serons-nous dans cinq ans? Notre vocation première consiste à garantir et développer à l'échelle mondiale le leadership technologique et commercial de la Bavière en matière de capteurs de mesure et d'asservissement. Cela se décline par toute une série de mesures visant à connecter les entreprises entre elles, accroître le potentiel d'innovation, développer nos compétences, étendre notre notoriété, créer de l'emploi, stimuler la création d'entreprises et disposer d'une maind'œuvre qualifiée.

\section{Le facteur humain au cœur du réseau}

Si une grande entreprise possède tous les outils requis en matière d'innovation, il n'en va pas de même pour les PME qui ne disposent pas nécessairement d'un département de R\&D ou d'un réel service du personnel et ne peuvent pas consacrer suffisamment de moyens à la formation initiale ou continue. Nous pouvons apporter une aide non seulement dans ces domaines, mais aussi en termes de financement, de marketing et vente, c'est-à-dire que nous mettons sur pied des demandes de financement, proposons des collaborations lors de salons ou assurons par ailleurs des prises de contacts à l'international via la société Bayern In- 
ternational [créée en 1995 à l'initiative du gouvernement de Bavière pour la promotion du commerce extérieur du Land; $\mathrm{SH}]$.

Notre approche repose sur la création d'une relation de confiance. C'est là que le facteur humain prend toute son importance. Car le réseau est constitué d'institutions anonymes; or pour les relier entre elles, vous avez besoin des responsables, des partenaires, des personnes. Quand vous disposez d'un réseau personnel, vous savez à qui vous adresser lorsque vous rencontrez un problème. Vous ne vous adressez pas à une institution, mais à la personne que vous connaissez en son sein. Voilà pourquoi le facteur humain est au cœur de notre stratégie. Car la confiance que vous avez dans les personnes impliquées permet de résoudre plus vite vos problèmes. L'une des missions de Sensorik est donc de créer la confiance, c'est-à-dire de tisser des liens entre les personnes. Et comment gagne-t-on la confiance de ses partenaires ? Par des rencontres. Quand vous savez qui est assis en face de vous, tout devient alors plus simple et plus rapide.

Il est capital de créer une relation de confiance pour pouvoir travailler ensemble. Lorsque nous rencontrons les dirigeants de PME pour la première fois, ils ne viennent pas nous exposer leurs projets futurs car ce qu'ils ont en tête constitue un potentiel en termes de chiffres d'affaires qu'ils ne souhaitent pas divulguer. Ils ne se confieront à nous que lorsque nous aurons réussi à instaurer une relation de confiance. Ce processus, qui mûrit pendant six mois voire un an, passe par le tutoiement et par un certain nombre de rencontres, procure à terme un sentiment fort : nous n'avons pas seulement trouvé un partenaire, mais aussi peutêtre un ami. L'aspect psychologique est déterminant.

\section{Evaluer en amont les risques liés à la propriété intellectuelle}

Quand un entrepreneur vient nous voir pour que nous l'aidions à lancer un produit ou un service innovant, nous signons d'abord un accord de confidentialité où nous nous engageons à ne pas révéler d'informations à des tiers. Toutes les personnes impliquées dans le projet doivent travailler dans ce sens, de sorte que l'entrepreneur conserve la maîtrise de son idée initiale. Lorsque nous avons plusieurs collaborateurs sur un tel projet, il est nécessaire de déterminer ce qui a trait à la propriété intellectuelle et de s'informer de la situation juridique en matière de brevets. Cela se traite toujours en amont, et plus tôt nous nous y consacrons, mieux nous pouvons éviter les conflits susceptibles de faire échouer le projet. En nous basant sur l'expérience des entreprises, nous cherchons à identifier les risques potentiels.

\section{Sensorik coordonne le travail en réseau sur l'ensemble de la chaîne de valeur}

Au cœur du réseau, Sensorik stimule les échanges entre les membres issus des milieux industriel et universitaire, les responsables en charge de la formation professionnelle continue, les structures de développement économique à l'échelon local et régional, le ministère bavarois de l'Economie, les CCI, les 
chambres de commerce extérieur ou encore les chambres de métiers et de l'artisanat. Bien que notre domaine d'activité se compose en grande partie de PME, le cluster comprend aussi de grandes entreprises dont l'expérience constitue une source d'inspiration. Dans ce contexte, divers acteurs participent à l'effort de R\&D : universités, PME, grandes entreprises disposant de leurs propres départements de R\&D, comme Siemens, Continental, Krones ou EADS, et centres de recherche, notamment les Instituts Fraunhofer de Munich et d'Erlangen. Toute la chaîne de valeur dans la production des capteurs de mesure et d'asservissement est donc ici représentée.

\section{Le réseau Sensorik réunit l'ensemble des acteurs de la chaîne de création de valeur}

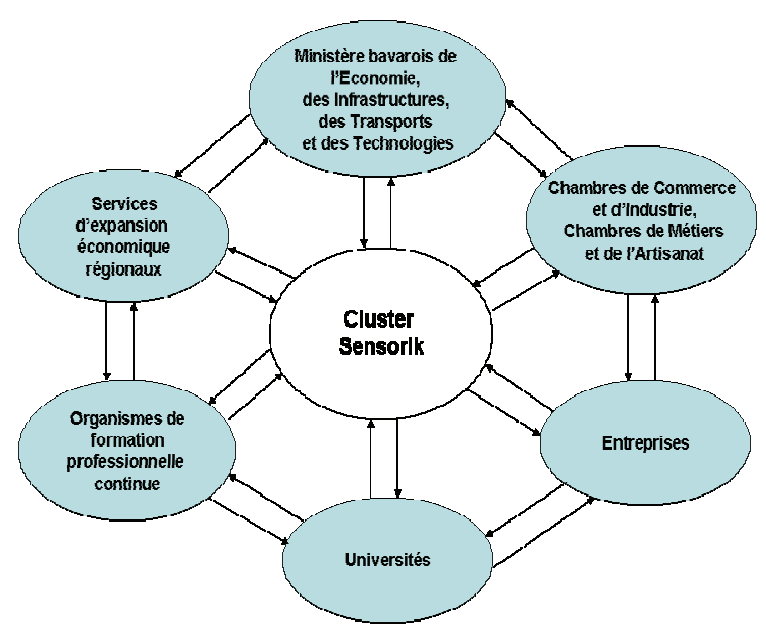

Source : Steigerwald, 2008.

\section{L'association au centre de l'organisation}

Notre structure organisationnelle concorde avec nos missions et objectifs. Le cluster comprend trois entités, avec au centre l'association Strategische Partnerschaft Sensorik e.V. - Partenariat stratégique-, véritable plateforme de mise en réseau et de suivi des acteurs, en charge des fonds propres et cofinancements. Les membres nous versent des cotisations: les universités contribuent à hauteur de $1000 €$ par an, tandis que certaines entreprises injectent jusqu'à $20000 €$ voire $30000 €$ par an, disposant en contrepartie de certains privilèges comme la réalisation d'études de faisabilité. Outre le financement, l'association se consacre aux ressources humaines, et notamment à la formation professionnelle initiale et continue, avec un pôle ad hoc à destination des membres du réseau et, plus généralement, de toute personne intéressée par les technologies de mesure et de commande. 


\section{Le Cluster Sensorik, structure institutionnelle de tête de réseau à l'échelle de la Bavière}

De l'initiative bavaroise est ensuite née une instance de représentation consacrée au secteur des capteurs. Cette seconde composante, le Cluster Sensorik, a pour mission de représenter les intérêts du Land de Bavière au niveau fédéral et international. L'entité recouvre toute l'activité marketing et l'organisation de salons et foires technologiques. Elle encourage les transferts de technologies et participe à la constitution de dossiers dans le cadre de demandes de soutien financier.

\section{La structure organisationnelle du partenariat stratégique Sensorik}

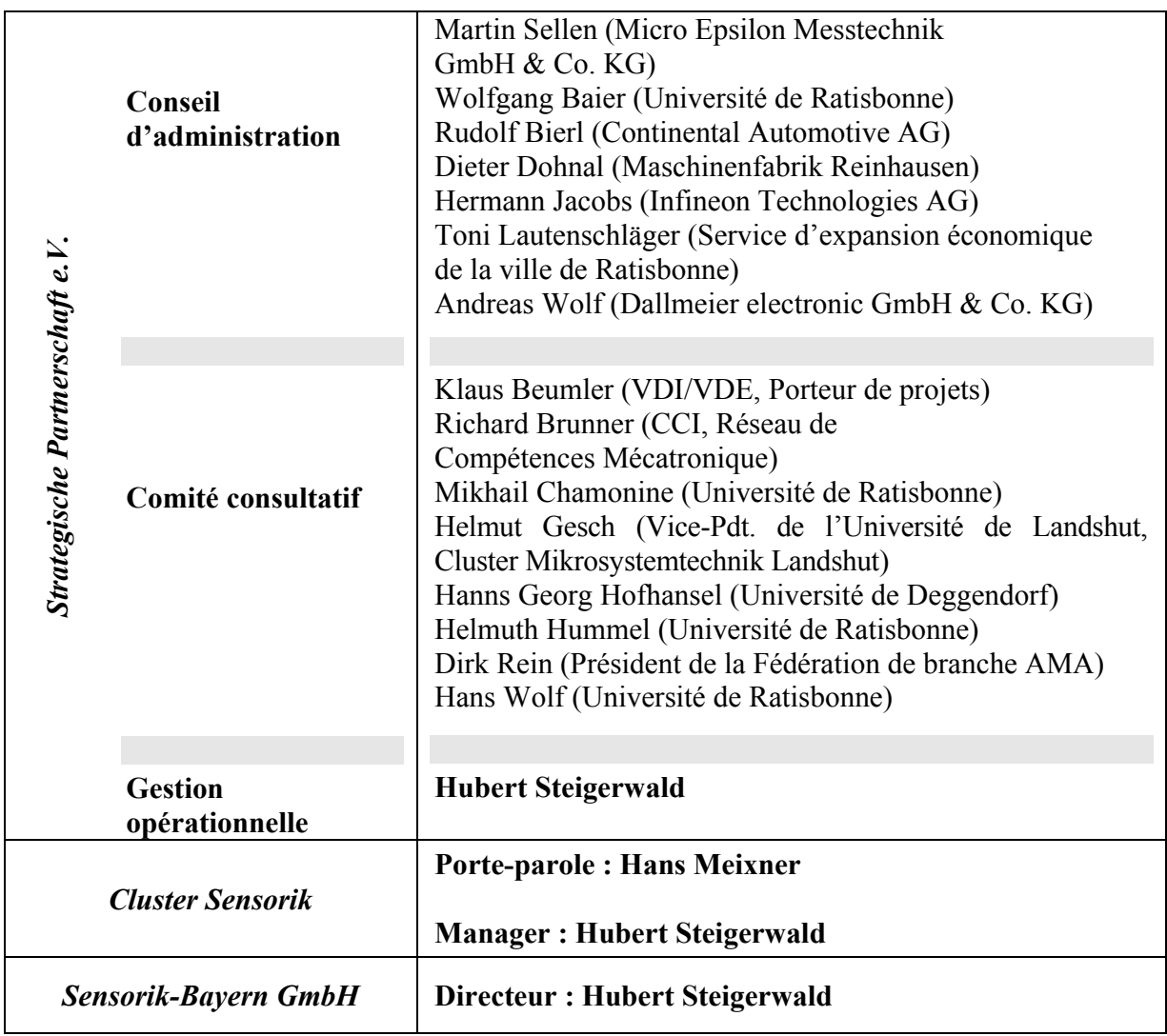

Source : Steigerwald, 2008.

\section{Sensorik-Bayern $\mathrm{GmbH}$ : un centre de compétences éminent}

Le troisième pilier de l'organisation, Sensorik-Bayern $G m b H$, agit pour ainsi dire comme une filiale de l'association. Créée par les membres du Partenariat Stratégique, elle est dédiée à tout ce qui a trait à l'innovation : $\mathrm{R} \& \mathrm{D}$, études 
de faisabilité, projets de partenariats, management de l'innovation... La gestion opérationnelle de Sensorik-Bayern $\mathrm{GmbH}$ me revient. Il en va de même pour les deux autres entités constitutives du réseau, ce qui crée de facto une passerelle entre les instances organisationnelles du cluster Sensorik. Par ailleurs, la composition du conseil d'administration et du comité consultatif de l'association, principal organe de Sensorik, témoigne des bases solides de notre partenariat science/industrie : des dirigeants d'entreprise y côtoient des professeurs d'université, un représentant des collectivités locales, un membre de la chambre de commerce et d'industrie, le dirigeant de la fédération de branche AMA ainsi qu'un représentant de la structure commune de l'Association allemande des ingénieurs et de la Fédération du secteur électrotechnique/électronique (VDI/VDE).

\section{La gestion des ressources humaines est primordiale}

L'association s'occupe des questions ayant trait à la gestion du personnel. Comme je l'ai évoqué, le facteur humain est capital : le succès repose sur les individus. Si vous avez des collaborateurs motivés, vous pouvez être assuré de la réussite de votre entreprise. Nous avons donc mis en place un pôle de personnel qualifié regroupant étudiants et personnes en quête d'emploi. Notre objectif n'est pas de mettre les entreprises en concurrence les unes avec les autres. Ces dernières nous exposent leurs besoins pour que nous puissions les aider à embaucher des personnes correspondant parfaitement aux profils recherchés. Comme nous recevons de nombreuses candidatures, nous procédons à des entretiens et réalisons une présélection. Nous aidons par ailleurs le candidat à améliorer la structure de son $\mathrm{CV}$. Ce service créé en 2007 nous a déjà permis de procéder, avec succès, à des recrutements pour le compte d'entreprises. Les personnes nouvellement en poste savent de qui elles tiennent leur emploi, ce qui nous offre un autre type d'accès aux entreprises. Mais cela n'est qu'un élément dans le maillage entre acteurs, qui demeure secondaire par rapport à l'aspect technologique. Le pôle de ressources humaines nous permet par ailleurs d'employer des personnes dont l'entreprise a procédé à des délocalisations. Flexibles, elles peuvent accepter de mettre leurs compétences à disposition des PME sur une base temporaire, ces dernières ne souhaitant pas recruter de tels experts à long terme, mais seulement dans la phase de développement d'un nouveau produit ou d'un nouveau service. Il s'agit à la fois de flexibilité pour les PME et de stabilité pour les salariés de notre pôle. La coexistence de la flexibilité et de la stabilité représente aujourd'hui un réel défi.

\section{Actions de formation destinées à constituer un potentiel de main-d'œuvre}

Nous voulons éveiller l'intérêt des étudiants pour les technologies de mesure et d'asservissement. Dans cette optique, nous organisons des présentations dans les universités, nous démarchons auprès des étudiants et contribuons à rendre ce do- 
maine de recherche attractif. Nous avons instauré une sorte d'université d'été où pendant une semaine chaque année, étudiants et collaborateurs d'entreprises sont formés, initiés à la problématique et apprennent mutuellement à se connaître. De grandes sociétés se joignent à cet événement pour évaluer le potentiel en termes de recrutement. Nous regroupons enfin, dans nos académies de formation initiale et continue, les universités du réseau situées dans un rayon de 100 à $120 \mathrm{~km}$.

\section{La SARL en support à la recherche et au transfert de technologies}

Au sein de la $\mathrm{GmbH}$, nous disposons d'un pôle de compétences dédié à la R\&D dans les technologies de mesure et d'asservissement, où nos propres physiciens, électrotechniciens et biochimistes font de la recherche pour le compte des entreprises. Nos employés savent que nos ressources sont limitées et connaissent leurs interlocuteurs dans les universités bavaroises. Toujours pour le compte des entreprises, nous déterminons le facteur coût et le facteur temps en matière d'innovation, partant du principe que si vous n'êtes pas assez rapide, vous serez vite dépassé par vos concurrents. Ces deux facteurs s'appliquent aussi aux universités, ce qui ne convient pas nécessairement à tout professeur ou à toute unité de recherche. Notre mission est alors de faire en sorte que tous travaillent ensemble dans cette même perspective...

Nous proposons en outre des études de faisabilité : un entrepreneur a une idée qui doit ensuite être formulée dans un dossier de demande de subvention, transmis à un porteur de projets, ici VDI/VDE, avec qui nous sommes régulièrement en contact et qui réalise une présélection. VDI/VDE fait ensuite parvenir la demande à l'échelon concerné (Land, Bund ou UE). Les financements de l'UE ne sont cependant pas adaptés aux besoins des PME car les délais de constitution et de traitement des dossiers sont trop longs pour des entreprises qui, pour survivre, doivent produire de l'innovation à court terme. Ainsi nous n'utilisons les programmes européens que pour les questions relatives aux infrastructures. Nous aidons enfin les PME en matière de management de l'innovation, problématique que nous intégrons par ailleurs à notre offre de formation.

\section{Un marketing au niveau régional et international...}

Le Cluster Sensorik, dernière composante du réseau, a quant à lui pour vocation d'accroître la visibilité de notre domaine d'activité, dans une stratégie plus globale de promotion du site économique de la Bavière. Nous agissons non seulement dans la région de Ratisbonne, de Munich, voire à l'échelle de la Bavière, mais aussi à l'international (Chine, Mexique...), où nous informons les entreprises étrangères des possibilités de collaboration avec notre réseau. Nous organisons des salons, effectuons des présentations en laboratoire, réalisons des actions de marketing pour nos membres, assurons les connexions entre nos membres et mettons sur pied des dossiers de demande d'aide financière. Dans le 
cadre de salons comme la Hannover Messe ou le salon Sensor + Test de Nuremberg, nous mettons en place des stands communs financés par les cotisations des membres, qui permettent à ces derniers de se rencontrer. Ces stands, toujours bien visités, apportent une meilleure visibilité aux PME. Par exemple, la technologie des capteurs étant très développée dans le secteur automobile, nous avons fait réaliser par nos membres issus de ce secteur une voiture de course équipée de divers capteurs. Présentée lors du salon Sensor + Test de 2008, cette voiture de Formule 1 a retenu l'attention de tous. Elle concentrait les innovations d'EMZ, Hofmann Leiterplatten, Infineon, Micro-Epsilon, PCO. Imaging, PreSens et Siemens VDO. Nous pensons par ailleurs qu'il est dans l'intérêt de notre réseau de prendre part à l'orientation de la politique européenne des clusters : c'est dans cette optique qu'à la fin de l'année 2008, j'ai déposé une candidature en vue d'intégrer le European Cluster Policies Group (ECPG).

\section{...en vue d'accroître la notoriété de Sensorik}

Notre stratégie marketing s'est avérée efficace: en 2007, nous avons pu rassembler plus de 2000 participants dans le cadre d'une cinquantaine de manifestations. Ces dernières permettent par ailleurs d'obtenir un retour direct des responsables politiques, fortement enclins à promouvoir les technologies de mesure et d'asservissement à l'échelle du Land (stratégiques en matière de communication). Nous organisons enfin des forums technologiques où des responsables d'entreprise, munis d'un cahier des charges, viennent exposer les difficultés techniques qu'ils rencontrent sur sept ou huit sujets différents en vue d'une coopération. Il est important pour nous, en termes de collaboration et de confiance, que les acteurs se réunissent, s'échangent, voire se rencontrent en dehors du travail : ils sont ainsi plus disposés à demander de l'aide à un membre du réseau. Nous réalisons aussi la promotion de notre cluster dans la presse, ce qui nous permet d'améliorer notre notoriété et de recevoir des demandes d'aides supplémentaires de la part d'entreprises.

\section{Un cluster labellisé « réseau de compétences »}

L'intégration de notre Partenariat Stratégique au programme fédéral des « réseaux de compétences » (Kompetenznetze) a eu un effet positif en termes de notoriété. Il s'agit là d'un véritable label en matière de durabilité, de qualité, de transferts technologiques, de promotion de l'innovation et de coopération. Cela nous a permis de communiquer vers l'extérieur et d'asseoir notre présence à l'échelle fédérale. La qualité et la diversité de nos services ont par ailleurs été confirmées par les résultats d'une évaluation menée au premier semestre 2008 par l'Institut Fraunhofer pour la Recherche sur les Systèmes et l'Innovation (ISI) de Karlsruhe pour le compte du ministère bavarois de l'Economie. Le 
sondage réalisé à cette occasion a donné lieu à un taux de retour exceptionnellement élevé (54\%), ce qui atteste du degré de satisfaction de nos membres, mais aussi du fait que les acteurs se connaissent au sein d'un réseau relativement restreint.

\section{Consolider les acquis et la chaîne de valeur}

Nous entrons dans une phase de consolidation durant laquelle nous allons continuer de nous développer en nous inspirant de ce que nous avons déjà réalisé ces deux dernières années. Dans un premier temps, nous allons mener à bien nos projets actuels car nous voulons prouver qu'à partir d'une idée, nous sommes capables d'aider les entreprises à produire de l'innovation malgré les nombreuses étapes à franchir. L'innovation suppose qu'il existe un besoin commercial : à l'écoute de la demande, nous vérifions constamment l'état et les perspectives du marché ainsi que la situation juridique en matière de brevets. Dans un second temps, nous valorisons nos thèmes de recherche en vue de trouver de nouveaux membres susceptibles de renforcer l'ensemble de la chaîne de valeur. Enfin, nous souhaitons acquérir de nouveaux projets afin de développer l'activité de notre SARL.

\section{La «crise»: une opportunité pour réaliser de nouvelles conquêtes technologiques}

La crise financière et économique actuelle pèse, bien sûr, sur certaines entreprises de notre réseau. En raison de la frilosité des banques, certaines, même les mieux gérées, peuvent rencontrer des problèmes passagers de liquidité. Nous y voyons une raison supplémentaire de venir en aide à nos membres et de les soutenir économiquement. Après avoir appréhendé les conditions d'ordre technique et commercial en vigueur dans les entreprises concernées, mon équipe s'attelle activement à la recherche de soutiens publics : elle s'engage notamment à obtenir des subventions au niveau du Land ou de l'Etat fédéral tout en dégageant et en activant des aides complémentaires. Je suis, pour ma part, fortement impliqué dans la promotion des PME à l'échelle fédérale en tant que membre, depuis 2008, du jury du programme ZIM (Zentrales Innovationsprogramm Mittelstand) mis en place par le ministère fédéral de l'Economie. Le recours efficace aux aides publiques permettra à notre organisation et à nos membres de bénéficier, pour l'après-crise, d'un positionnement stratégique sur des projets à forte densité technologique. Car, plus fondamentalement, nous considérons la situation actuelle comme le moment idéal pour innover. Nos membres doivent pouvoir profiter pleinement de notre offre de services. L'approche que nous partageons avec eux consiste à voir dans la «crise » l'opportunité d'aborder de nouveaux domaines technologiques et de jeter les bases d'un avenir serein en termes économiques. 


\section{De nouveaux membres et des projets prometteurs}

Sensorik évolue dans un formidable processus de développement et de croissance, quelque peu à contre-courant de la tendance économique actuelle (ce qui nous réjouit particulièrement en tant que plate-forme de réseau). D'un côté, nous connaissons depuis de nombreuses semaines un afflux exceptionnel de nouveaux membres cotisants (grandes entreprises et PME). De l'autre, à travers ce processus de développement et de croissance, nous nous détachons clairement d'autres réseaux de la Cluster-Offensive.

Nous cherchons à nous rapprocher des entreprises en nous orientant clairement vers des projets high-tech pilotes et porteurs d'avenir (capteurs magnétiques innovants pour les systèmes de gestion du trafic, mesures d'épaisseur de couches térahertz ou encore dispositifs de pointage munis d'une caméra dans le domaine de la chirurgie mini-invasive). Par ce biais, nous apportons notre aide aussi bien aux grandes entreprises industrielles bavaroises qu'à de nombreuses PME du secteur qui, grâce à leur offre de niche, parviennent à se hisser au rang de leader mondial. Via des coopérations science/ industrie, notre objectif plus global est de continuer de développer avec succès un site bavarois des capteurs de mesure et de commande au potentiel prometteur.

\section{L'orientation stratégique de Cluster Sensorik : un vecteur de croissance}

Notre plate-forme de réseau bénéficie également de ce processus de développement et de croissance, dont la garantie future passe par notre positionnement stratégique en tant que cluster bavarois : en février 2009, nous nous sommes vu remettre par le secrétaire d'Etat parlementaire auprès du ministère fédéral de l'Economie, Hartmut Schauerte, l'un des prix du concours «Réseaux de compétences 2009 - Pour un développement pérenne des réseaux » qui s'inscrit dans l'initiative «Réseaux de compétences » du ministère. Je souhaite préciser par ailleurs que dans le cadre de l'évaluation menée pour le compte du ministère bavarois de l'Economie, notre cluster a obtenu la meilleure note, aussi bien de la part de notre évaluateur que des membres et acteurs du réseau.

\section{Le facteur humain, unique dénominateur commun de la réussite}

Bien que notre réseau se développe avec succès suivant le modèle que je vous ai exposé, cet exemple est difficilement transposable. Chaque cluster possède en effet des missions qui lui sont propres et connaît une situation de départ différente. Un seul élément commun demeure cependant: le facteur humain, dont la contribution à la réussite des réseaux est essentielle.

Traduction de Solène HAZOUARD 


\section{Indications bibliographiques}

BALZEREIT J., Innovationsfaktor Kooperation - Ein Gewinn für Wirtschaft und Wissenschaft!, Chambre de Commerce et d'Industrie de Ratisbonne, Ratisbonne, 2007

BAYERISCHES STAATSMINISTERIUM FÜR WIRTSCHAFT, INFRASTRUKTUR, VERKEHR UND TECHNOLOGIE, Cluster-Offensive Bayern - Im Netzwerk zum Erfolg, Bayerisches Staatsministerium für Wirtschaft, Infrastruktur, Verkehr und Technologie, Munich, 2008

BouRgeOIS I., «Bavière : la patiente construction d'une économie performante», in BouRGEOIS I. (dir.), Allemagne : compétitivité et dynamiques territoriales, CIRAC, Cergy-Pontoise, 2007

«The concept of cluster and cluster policies and their role for competitiveness and innovation: Main statistical results and lessons learned», SEC(2008) 2637, Europe INNOVA/PRO INNO Europe paper $\mathrm{n}^{\circ}$ 9, Document de travail des services de la Commission européenne - Annexe à la communication du 17.10.2008 COM(2008) 652: Vers des clusters de classe mondiale dans l'Union européenne: mise en ouvre d'une stratégie d'innovation élargie

DOMDEY H., «Le pôle biotech de Munich : une "culture de l'innovation"», Regards sur l'Economie allemande, $\mathrm{n}^{\circ} 86 / 08$, mai 2008, p. 19-24

BAYERISCHES STAATSMINISTERIUM FÜR WIRTSCHAFT, INFRASTRUKTUR, VERKEHR UND TECHNOLOGIE, Offensive des clusters bavarois [en ligne] : http://www.cluster-bayern.de [consulté le 07/01/10]

BAYERN INTERNATIONAL (BAYERISCHE GESELLSCHAFT FÜR INTERNATIONALE WIRTSCHAFTSBEZIEHUNGEN MBH), Bayern international [en ligne] : www.bayern-international.de [consulté le 07/01/10]

DIRECTION INTERMINISTÉRIELLE DE LA COMPÉTITIVITÉ DES TERRITOIRES (DIACT), Les pôles de compétitivité [en ligne] : www.competitivite.gouv.fr [consulté le 07/01/10]

Cluster LeistungSElEKTRONIK, Cluster Leistungselektronik [en ligne]: www.cluster-bayernleistungselektronik.de [consulté le 07/01/10]

CENTER FOR STRATEgy AND COMPETITIVEnESS, European Cluster Observatory [en ligne]: www.clusterobservatory.eu [consulté le 07/01/10]

FRAUNHOFER INSTITUT FÜR SYSTEM- UND INNOVATIONSFORSCHUNG (ISI), Fraunhofer ISI [en ligne]: http://cms.isi.fraunhofer.de [consulté le 07/01/10]

VDI/VDE INNOVATION + TECHNIK GMBH, Kompetenznetze Deutschland [en ligne]: www.kompetenznetze.de [consulté le 07/01/10]

STRATEgische PARTNERSCHAFt SENSORIK E.V., Sensors made in Bavaria-Sensorik-Bayern-GmbH [en ligne] : www.sensorik-bayern.de [consulté le 07/01/10]. 
- IV -

LES PME AU COEUR DES PROCESSUS D'INNOVATION 



\title{
Fonction des PME dans le système d'innovation allemand : la politique de soutien au Mittelstand
}

\author{
Marianne KULICKE
}

Selon les évaluations du Centre pour la recherche européenne (ZEW), on observe ces dernières années une stagnation, voire un recul du nombre de PME menant des activités de R\&D outre-Rhin. Marianne Kulicke, chef de projets à l'Institut Fraunhofer de recherche sur les systèmes et l'innovation (ISI) de Karlsruhe, s'intéresse dans la présente contribution aux cinq catégories de PME définies par le ZEW en fonction de leur effort de $R \& D$ ainsi qu'à la politique de soutien au Mittelstand, inscrite depuis 2006 dans la stratégie "Hautes Technologies » du gouvernement fédéral.

\section{La place des PME en Allemagne}

En Allemagne, le Mittelstand a traditionnellement une importance économique majeure en termes de diversité des structures et de compétitivité dans les secteurs du commerce, de l'artisanat, de l'industrie, de la production et des services ainsi que dans celui des professions libérales. Ce terme désigne généralement les petites et moyennes entreprises (PME) au sens défini par l'UE', c'est-à-dire les entreprises qui emploient moins de 250 salariés et dont le chiffre d'affaires annuel est de 50 millions $€$ au maximum. Certaines études empiriques et statistiques englobent aussi dans cette catégorie les entreprises comptant jusqu'à 500 salariés $^{2}$. Ce plafond de 500 salariés, souvent utilisé par le passé, garde des répercussions aujourd'hui. Ainsi, de nombreux programmes de financement de la banque KfW, l'établissement public de soutien aux PME, appliquent ce nombre maximal de salariés.

En Allemagne, l'importance économique du Mittelstand est telle que près de 3,2 millions de PME emploient 20,8 millions de salariés (soit environ $70 \%$ des salariés), et représentaient en 2006 un chiffre d'affaires d'environ 1730 milliards $€$. Près de la moitié $(47 \%)$ de la valeur ajoutée brute nationale provient des PME, qui par ailleurs forment $83 \%$ des apprentis. A titre de comparaison, 8300 grandes entreprises emploient 5,7 millions de salariés, et représentent un chiffre d'affaires de 2608 milliards $€$.

La grande majorité des PME opère dans les secteurs du commerce et de l'artisanat ainsi que dans celui des services de proximité. Selon les estimations du Centre pour la recherche européenne (ZEW), environ 140000 PME travaillent dans l'industrie manufacturière et dans les secteurs à haute technologie. Ces dernières années, le nombre de PME concernées a toutefois connu une progression tout aussi faible que le taux d'entreprises innovantes

\footnotetext{
${ }^{1}$ Fixé à l'article 2 de l'annexe à la recommandation 2003/361/EG. Voir Communautés européennes (2006).

${ }^{2}$ Voir BMWI (2007, p. 9 sv.).
} 
(c'est-à-dire la part des entreprises réalisant des produits ou des processus innovants). Le taux d'entreprises innovantes ${ }^{3}$ est même en léger recul. Il était encore de $47 \%$ en 2004, et a atteint $45 \%$ en 2005 . Il est actuellement d'environ $46 \%$. Ce chiffre varie toutefois en fonction des secteurs d'activité. En 2005, le ZEW indiquait ainsi un taux de $58 \%$ pour les entreprises de l'industrie manufacturière (ce taux était encore de $67 \%$ en 1999, et a connu un léger recul ces dernières années), de $55 \%$ pour les services high-tech (contre un taux de $67 \%$ en 1998 , et oscillant entre 52 et $55 \%$ depuis 2002) et de $29 \%$ pour les autres types de services (soit un net recul depuis 2000, où ce taux était de $45 \%$ ). La tendance au repli s'est amorcée dès les années 1980, entraînant une concentration des activités de R\&D sur un groupe de plus en plus restreint de $\mathrm{PME}^{4}$.

Les acteurs politiques notamment en concluent que la capacité d'innovation des PME et donc de l'économie nationale allemande diminue et que des mesures d'aide sont indispensables pour soutenir la force d'innovation des PME.

\section{Activités d'innovation des PME}

Ces dernières années, le personnel de R\&D employé par les PME (définies ici comme les entreprises de 500 salariés au maximum) connaît également une diminution en valeur absolue. Or, le nombre de personnes employées au total par les entreprises dans ce secteur n'a cessé de croître sur la durée. Dans ses enquêtes régulières sur la $\mathrm{R} \& \mathrm{D}$, l'Association des donateurs pour la science allemande (Stifterverband für die deutsche Wissenschaft) observe l'évolution suivante :

Tableau 1 : Personnel de R\&D (tous secteurs confondus)

\begin{tabular}{|c|c|c|}
\hline Année & $\begin{array}{c}\text { Personnel R\&D employé dans } \\
\text { des PME (500 salariés max.) }\end{array}$ & $\begin{array}{c}\text { Personnel R\&D employé dans } \\
\text { l'ensemble de l'industrie }\end{array}$ \\
\hline 1997 & 56500 & 286270 \\
\hline 2001 & 54000 & 307257 \\
\hline 2005 & 52000 & 304500 \\
\hline
\end{tabular}

Source : Stifterverband für die deutsche Wissenschaft (2006) (2007).

Alors que les PME ont par ailleurs un poids économique important sur le marché de l'emploi et qu'elles apportent une contribution considérable en matière de formation et de création de valeur ajoutée, elles ne représentaient en 2005 qu'une faible part de l'ensemble des dépenses de R\&D de l'industrie allemande. Cette année, sur un total de 44,72 milliards $€$ de dépenses de R\&D

\footnotetext{
${ }^{3}$ Source des données suivantes : Rammer (2007, p. 1 sv.).

${ }^{4}$ Voir : Stifterverband (2006), IW Consult GmbH (2006, p. 28).
} 
pour l'ensemble des entreprises, 41,18 milliards $€$ sont le fait des grandes entreprises (250 salariés et plus). Seuls $7,9 \%$ de ces dépenses, soit tout de même une valeur absolue de 3,5 milliards $€$, émanent des PME. Si l'on rapporte les dépenses de $R \& D$ à la taille et donc aux moyens financiers des entreprises, il apparait que la sous-catégorie des entreprises de moins de 100 salariés contribue dans des proportions considérables aux prestations de R\&D. Le graphique cidessous montre cependant qu'en Allemagne l'essentiel des dépenses dans ce domaine est assumé par les grandes entreprises, en particulier celles de 10000 salariés et plus.

Graphique 1 : Part des différentes catégories d'entreprises dans l'ensemble des dépenses de R\&D de l'industrie en 2005

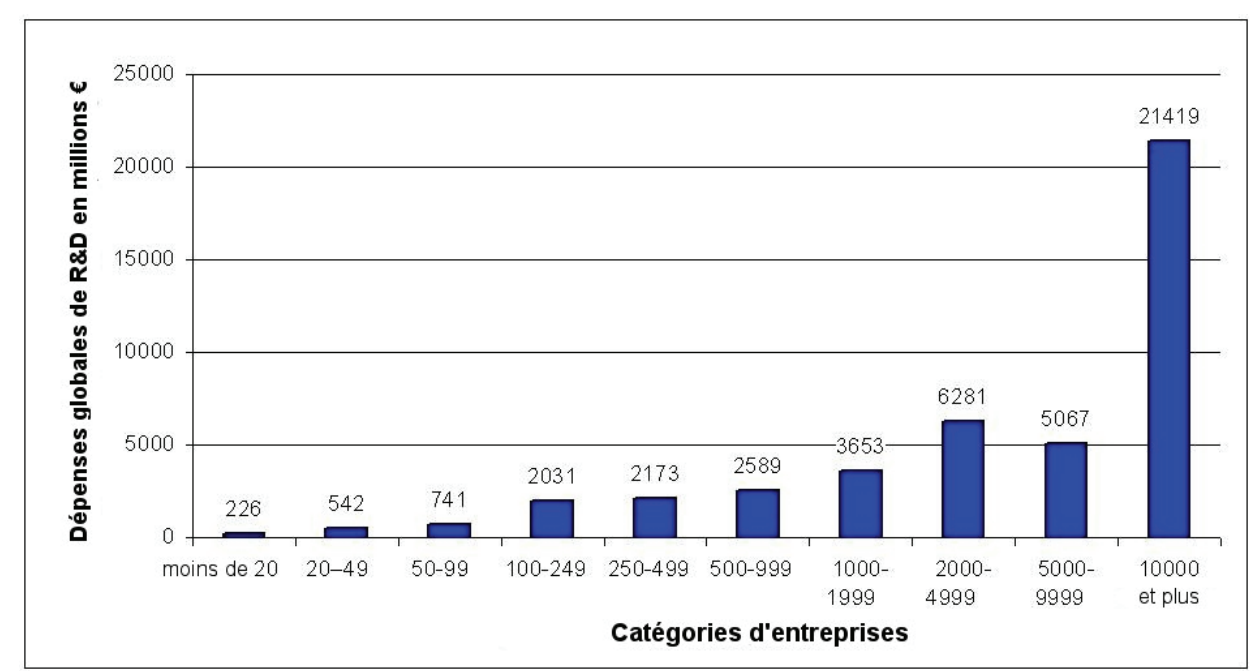

Source : Stifterverband (2007, p. 27).

Dans une étude consacrée à l'innovation dans les PME de l'industrie manufacturière et dans les secteurs des services high-tech, le $\mathrm{ZEW}^{5}$ définit les cinq catégories d'entreprises suivantes :

(1) Les start-up high-tech, c'est-à-dire de jeunes entreprises (5 à 10 années d'existence selon les secteurs) ayant un taux élevé de R\&D (au moins $10 \%$ du chiffre d'affaires consacré aux activités de R\&D, ce chiffre étant en général nettement plus élevé) et fortement tournées vers l'export. La grande majorité d'entre elles sont des entreprises de services à faible croissance. Le ZEW estime le nombre de start-up high-tech à environ 5000 , dont $90 \%$ d'entreprises de services innovantes. Les défis auxquels elles sont confrontées consistent à assurer leur financement et à trouver

\footnotetext{
${ }^{5}$ Voir Rammer (2005, p. 1-2); Version intégrale de l'étude : Rammer et al. (2006).
} 
de nouveaux débouchés. L'étude du ZEW en déduit deux types de besoins essentiels : un bon fonctionnement du marché du capital-risque et un allègement des coûts de recherche.

(2) Les prestataires de $\mathbf{R} \& \mathbf{D}$, qui sont des entreprises généralement plus anciennes (au moins 5 années d'existence, mais le plus souvent plus de 10 ), dotées d'un taux de $R \& D$ très élevé et effectuant des travaux de R\&D pour le compte d'autres entreprises. Leur nombre est actuellement estimé à environ 2000 . De nombreuses entreprises de recherche privée d'intérêt public ou purement privées ont été créées, en particulier en Allemagne de l'Est, à la suite de la restructuration du système de recherche après la réunification. Ces sociétés mènent des recherches sur commande, pour le compte d'autres PME ou de grandes entreprises qui ne font pas de R\&D, à l'aide de financements privés ou bien dans le cadre de programmes publics de financement ${ }^{6}$. La difficulté de ces entreprises consiste à dépendre de la demande de l'industrie, et donc fortement des cycles de R\&D des acheteurs. Ces entreprises prestataires de $R \& D$ dénoncent souvent des distorsions de concurrence dues au financement public de coopérations de recherche orientées application entre des entreprises et des centres de recherche financés sur des fonds publics (universités, instituts Fraunhofer, laboratoires Leibniz, instituts MaxPlanck, etc.), mais beaucoup d'entre elles en bénéficient aussi largement.

(3) Les PME ayant des activités de recherche régulières : ces entreprises constituent le cœur des PME technologiques en Allemagne; elles assument clairement, avec plus de trois milliards $€$ annuels, plus de la moitié de toutes les dépenses de R\&D des PME allemandes. Elles représentent le plus important groupe cible et le principal bénéficiaire de la plupart des programmes de promotion de l'innovation de l'Etat fédéral allemand, des Länder et de l'UE dédiés aux PME. Le ZEW estime leur nombre à environ 29000 . Les principales difficultés de ce type de PME consistent à assurer la régularité de leurs travaux de R\&D par autofinancement et à disposer du personnel permettant de faire face aux évolutions conjoncturelles.

(4) Des PME parvenant à innover sans avoir d'activités de R\&D régulières : il s'agit de suiveurs innovants, qui reprennent les idées de produits et de procédés inventés par d'autres entreprises et les commercialisent, souvent en les adaptant à une clientèle ciblée. Avec au moins 50000 entreprises, elles représentent le deuxième groupe de PME innovantes. En matière d'innovation, il s'agit surtout pour ces entreprises d'asseoir leur position face à la concurrence en reprenant très vite des

\footnotetext{
${ }^{6}$ Voir Kulicke et al. (2006); Lo et al. (2006); Koschatzky et al. (2003).
} 
idées leur laissant suffisamment de débouchés potentiels dans des niches de marchés.

(5) Les PME ne parvenant pas à innover : dans l'industrie manufacturière et dans les secteurs des services high-tech, ces entreprises constituent la catégorie la plus importante (près de 55000 entreprises). Ces PME se caractérisent par une taille modeste (environ 60 salariés en moyenne) et par une rentabilité inférieure à celle des autres catégories. Elles sont très nombreuses à n'avoir réalisé aucune innovation (produits ou procédés) sur une durée de trois ans. Pour assurer leur compétitivité à long terme, ces entreprises doivent réussir à moderniser régulièrement leurs produits et services et être suffisamment rentables pour assurer leur survie sur le marché et, enfin, parvenir à intégrer le groupe (4) voire le groupe (3).

Le graphique 2 montre la répartition des PME selon ces cinq catégories. Il apparaît clairement que les trois quarts environ des PME font partie des deux groupes n'effectuant pas de R\&D par elles-mêmes. Les programmes publics d'aide aux PME visent donc notamment à mettre en place des mesures incitatives pour que davantage de PME mènent régulièrement des activités de recherche.

Mais il n'existe actuellement, à l'échelle fédérale comme dans la plupart des Länder, aucun dispositif général d'aide à la création d'entreprises high-tech par le biais de subventions. Ces dix dernières années, on a observé une focalisation sur les créations d'entreprises issues de laboratoires de recherche (réseaux de création principalement) et des entreprises créées dans une logique de croissance, en faisant appel au capital-investissement. Il n'y a pas de mesures spécifiquement dédiées aux prestataires de $\mathrm{R} \& \mathrm{D}$, qui ne sont par ailleurs que partiellement éligibles aux programmes de soutien à l'innovation existants.

Les PME menant des activités de recherche régulières sont au contraire les principaux bénéficiaires de la plupart des programmes de soutien à l'innovation dédiés aux PME, que ce soit au niveau du Bund, des Länder ou de l'UE. Elles n'ont en revanche qu'un accès limité aux grands programmes spécialisés de soutien à la recherche. Il n'existe pas non plus de dispositif à grande échelle spécifiquement dédié aux PME parvenant à innover sans effectuer de R\&D régulière; ces entreprises sont toutefois les principales cibles de diverses mesures d'incitation à la recherche et d'actions de conseil visant à leur donner les compétences pour adopter de nouvelles solutions technologiques. Les programmes proposés jusque-là n'ont toutefois pas encore permis à des entreprises de cette catégorie de rejoindre le cercle des PME innovantes. On peut dresser le même constat pour les PME ne parvenant pas à innover. Elles sont elles aussi la cible de diverses mesures d'incitation à la recherche, avec les mêmes résultats que ceux des PME parvenant à innover sans mener d'activités de $R \& D$ régulières. 
Graphique 2 : Nombre de PME pour chacune des cinq catégories dans l'industrie manufacturière et dans les secteurs des services high-tech (2003)

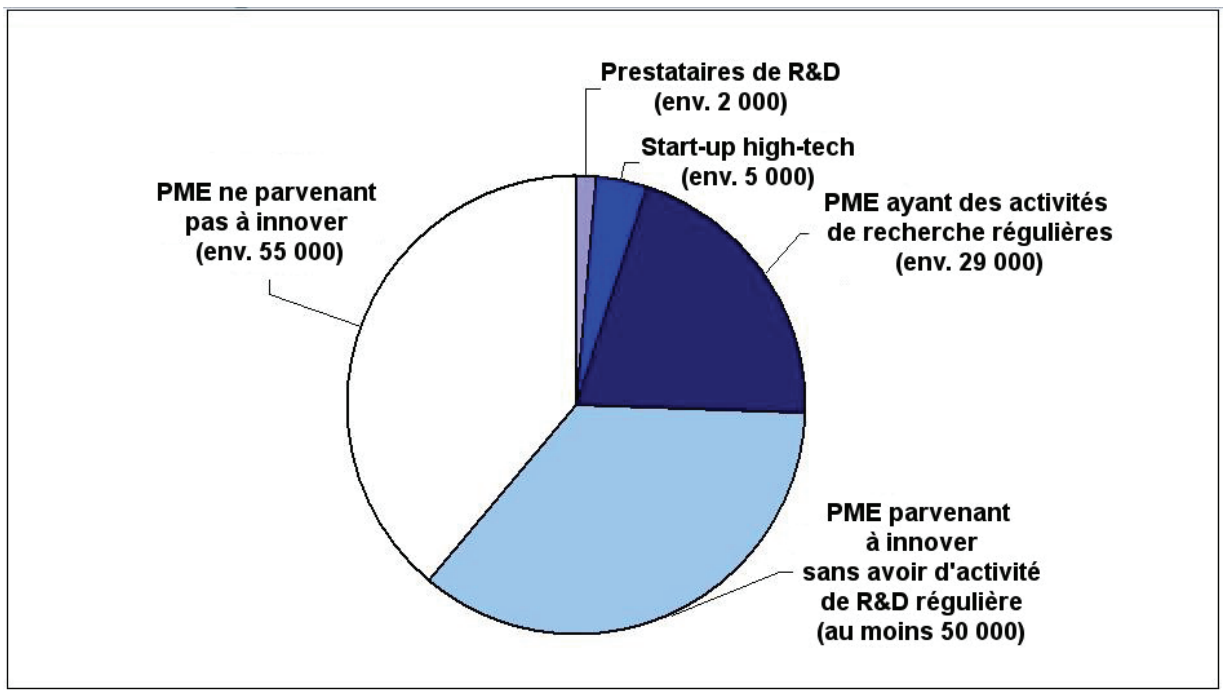

Source : Rammer (2005, p. 1-2).

La stratégie «Hautes Technologies» adoptée en 2006 par le gouvernement fédéral regroupe tout un ensemble d'activités et de programmes visant à renforcer la capacité d'innovation des PME. Ces dispositifs constituent pour partie le prolongement (modifié) de programmes existant depuis longtemps déjà ; mais de nouveaux instruments ont également été mis en œuvre afin de promouvoir la coopération entre science et industrie.

\section{La politique de soutien au Mittelstand}

\subsection{Inscription du soutien aux PME innovantes dans la stratégie « Hautes Technologies » du gouvernement fédéral}

La stratégie "Hautes Technologies 》 du gouvernement fédéral (publiée en 2006) met l'accent sur la politique de soutien aux PME innovantes comme élément essentiel du renforcement de la capacité d'innovation de l'Allemagne. L'objectif est de faire de l'Allemagne l'un des leaders mondiaux sur les principaux marchés d'avenir. Il est donc prévu d'investir 15 milliards $€$ pour la recherche et le développement au cours de la législature actuelle (d'ici 2009). Cette stratégie « Hautes Technologies » constitue globalement le prolongement de programmes qui existaient déjà et sont progressivement complétés par de nouveaux programmes. Elle vise en outre à rendre les conditions fiscales et le cadre réglementaire plus favorables aux entreprises et au système scientifique. Il 
existe par ailleurs au niveau fédéral tout un éventail de programmes de soutien de la KfW (instruments de financement: prêts, financement par du capitalinvestissement) avec un volume de crédit très important, prioritairement destiné à financer les PME d'une manière générale et non spécifiquement l'innovation, mais comportant aussi des mesures relatives à l'innovation. L'offre dédiée aux PME innovantes est complétée par un grand nombre de programmes de soutien dans les 16 Länder. La politique régionale d'aide connaît actuellement de profonds changements du fait des moyens émanant du FEDER et du FSE pour la période 2007-2013, qui augmentent considérablement la marge de manœuvre financière des Länder. On a ainsi observé dès fin 2007 et début 2008 le lancement de nouveaux programmes de promotion de l'innovation à l'échelon régional. Les moyens alloués à de tels programmes ont connu une hausse sensible comparativement aux années précédentes, surtout dans les nouveaux Länder, de même que dans certains anciens Länder comportant des régions confrontées à des reconversions industrielles (Basse-Saxe, Rhénanie du NordWestphalie). Ce phénomène, conjugué à une augmentation des recettes fiscales, a permis une augmentation considérable des actions régionales de promotion de l'innovation, qui avaient connu une forte chute dans le contexte de la récession économique de 2003-2004 et des réductions budgétaires.

La stratégie "Hautes Technologies» a été mise en place en tant que stratégie nationale du gouvernement fédéral ayant vocation à réaliser, par-delà les différentes administrations, une politique d'innovation coordonnée. On trouve au cœur de ce dispositif des stratégies d'innovation spécifiques dans 17 domaines technologiques et 5 grands domaines transversaux. La mise en œuvre des stratégies d'innovation ne se concentre pas sur quelques thèmes, mais couvre largement tous les domaines technologiques considérés comme « modernes » et porteurs d'avenir ${ }^{7}$. Des programmes thématiques de soutien à la $R \& D$ ont été lancés, qui servent de programmes cadre avec un horizon à 5 ou 10 ans.

Les cinq domaines transversaux sont :

- la coopération entre science et industrie à l'aide d'une stratégie transversale de clusters ;

- la valorisation et l'accès à la recherche ;

- l'échange de spécialistes ;

- l'aide à la création ;

- le soutien aux PME.

\footnotetext{
${ }^{7}$ Il s'agit de la recherche et des technologies médicales, des technologies de la sécurité, de la botanique, des technologies énergétiques, des technologies environnementales, des technologies de l'information et de la communication, des technologies de transport, des technologies aéronautiques, des technologies aérospatiales, des technologies maritimes, des services, des nanotechnologies, des biotechnologies, des technologies des microsystèmes, des technologies optiques, des technologies des matériaux et des technologies de la production. Voir : http://www.hightech-strategie.de/de/77.php [consulté le 06/09/10].
} 
Graphique 3 : La stratégie " Hautes Technologies » en Allemagne : tentative de synthèse

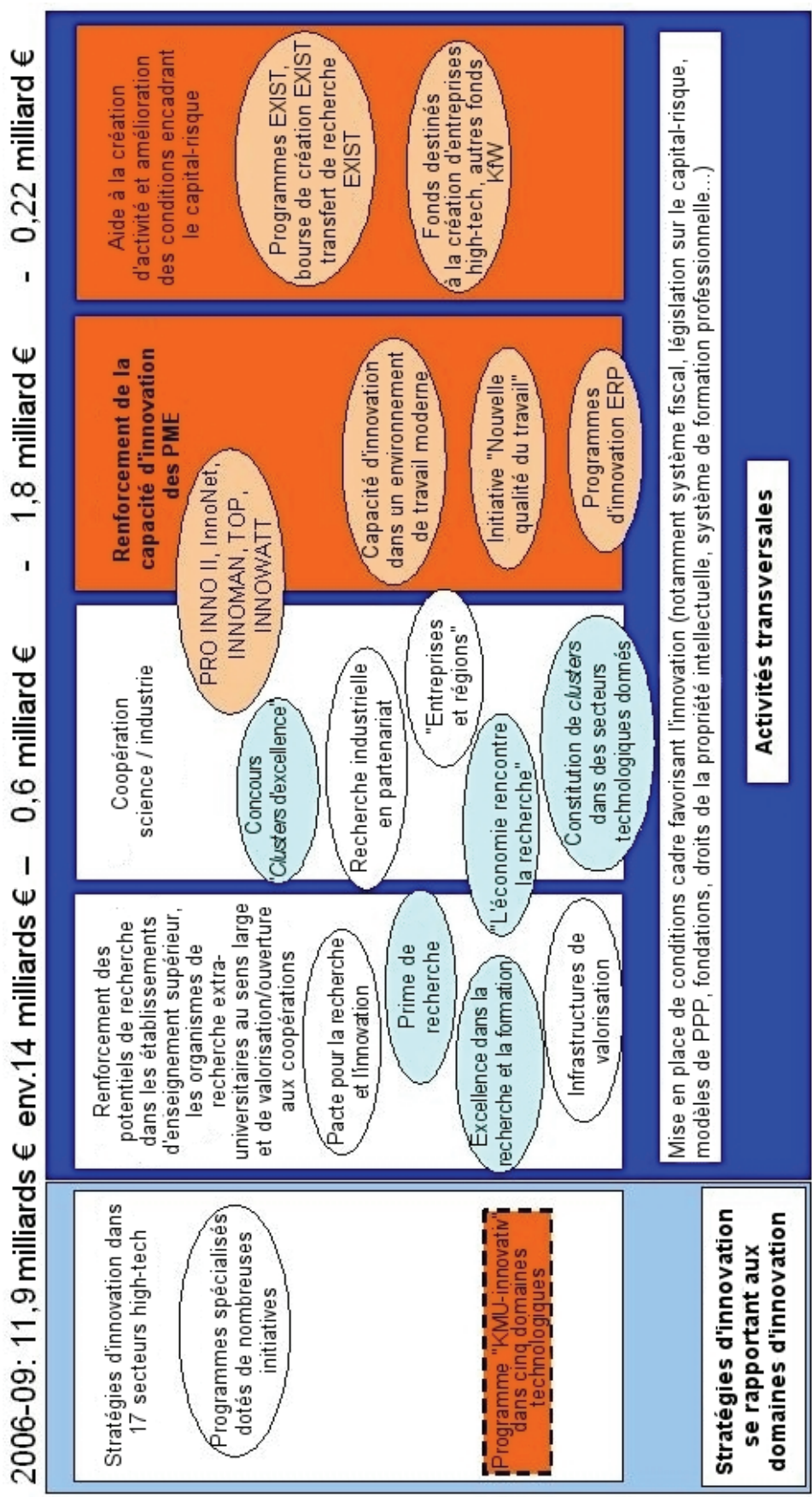


Le graphique 3 tente d'organiser les programmes et activités listés dans la copieuse présentation de la stratégie « Hautes Technologies ». Il faut souligner les points suivants :

- le soutien aux PME innovantes se concentre essentiellement sur les deux derniers domaines transversaux cités. Mais, au sein même des 17 domaines technologiques, la stratégie «Hautes Technologies» s'efforce de définir des conditions d'aide tenant compte des potentiels et des contraintes des PME. C'est notamment le souci des mesures «KMU-innovativ» dans cinq domaines technologiques, qui facilitent l'accès des PME aux programmes de soutien concernés ;

- les dispositions financières prévues (dont une partie est encore à l'étude) reflètent les priorités : de 2006 à 2009, 11,9 milliards $€$ doivent être consacrés aux stratégies d'innovation dans les 17 domaines technologiques ;

- l'activité transversale «renforcement des potentiels de recherche dans les établissements d'enseignement supérieur, les organismes de recherche extra-universitaires au sens large et de valorisation/ouverture aux coopérations » comporte, avec le Pacte pour la recherche et l'innovation, un renforcement des grands organismes scientifiques et de recherche financés sur des fonds publics ou recevant une dotation ${ }^{8}$. Un budget d'environ 14 milliards $€$ est prévu pour ces programmes et mesures ;

- l'activité transversale "coopération science-industrie» est nettement moins bien dotée, avec environ 600 millions $€$ pour la même période ;

- une enveloppe d'1,8 milliard $€$ est prévue pour le domaine « renforcement de la capacité d'innovation des PME», qui regroupe les principaux programmes de soutien aux PME innovantes ;

- la quatrième activité transversale vise l'aide à la création d'activité et l'amélioration des conditions encadrant le capital-risque. Les start-up high-tech en constituent le groupe cible ;

- le cinquième domaine des activités transversales vise à établir un environnement plus favorable à l'innovation (système fiscal, législation sur le capital-risque, modèles PPP, fondations, droits de propriété intellectuelle, système de formation professionnelle, etc.).

En matière d'aide aux PME, la stratégie « Hautes Technologies » met l'accent sur les domaines suivants :

- coopérations d'innovation visant à renforcer les activités de R\&D dans les entreprises et la mise en réseau de celles-ci avec les universités, les

\footnotetext{
${ }^{8}$ Il s'agit de la Communauté Helmholtz, de la Société Max-Planck (MPG), de la Société Fraunhofer (FhG), de la Communauté Leibniz (Communauté scientifique Gottfried Wilhelm Leibniz e.V., WGL) et de la Communauté allemande pour la recherche (DFG) en tant qu'organisme de promotion de la recherche, dont bénéficient également les universités. Voir : http://www.bmbf.de/de/3215.php [consulté le 06/09/10].
} 
centres de recherche et d'autres entreprises afin de développer de nouveaux produits, services et procédés; à cette fin, certains programmes de soutien (PRO INNO II en particulier, mais aussi NEMO, InnoNet et INNO-WATT) sont regroupés à partir de la mi-2008 en un nouveau dispositif, le «Programme central d'innovation pour le Mittelstand $»(\mathrm{ZIM})^{9}$. Il est prévu d'en faciliter l'utilisation et de l'adapter encore davantage aux contraintes des PME innovantes ;

- amélioration de la capacité d'innovation dans les PME (qualification, conseil) ;

- «KMU-innovativ» : plus grande participation de PME du secteur des technologies de pointe aux programmes spécialisés, bien dotés, du soutien à la recherche (p. ex. biotechnologies, nanotechnologies, technologies de la production);

- aide aux organismes externes de recherche industrielle, partenaires de recherche des PME dans les nouveaux Länder;

- simplification du processus de financement et amélioration des conditions cadre.

Ce dispositif ne dote toutefois pas l'Allemagne d'incitations fiscales favorisant l'innovation dans les (petites et moyennes) entreprises; seules des aides individuelles limitées sont prévues par les programmes dédiés au Mittelstand. Les programmes d'innovation mettent clairement l'accent sur le soutien à la coopération entre entreprises ainsi qu'entre entreprises et organismes de recherche (universités incluses).

Les mesures prévues pour la création d'entreprises innovantes dans le cadre de la stratégie « Hautes Technologies » sont les suivantes :

- aide à la création d'entreprises issues d'universités et d'organismes de recherche via les programmes EXIST - aide à la création d'entreprises issues de la science, bourse de création EXIST (préparation à la création), transfert de recherche EXIST (pour le développement des résultats de la recherche en phase de préparation à la création jusqu'à la viabilité sur le marché ; à partir de début 2008) ; Objectifs :

- établir une culture de la création dans les universités et les centres de recherche,

- accroître le transfert de technologie et la valorisation du savoir et des résultats de la recherche en créant des spin-off (jeunes pousses),

- renforcer les réseaux régionaux en soutenant ce type de créations d'activité,

\footnotetext{
${ }^{9}$ Voir BMWi (2008, p. 18-23).
} 
- augmentation des fonds destinés à la création d'entreprises high-tech pour soutenir l'investissement dans de nouvelles entreprises high-tech ;

- amélioration des conditions fiscales pour favoriser les investissements.

Dans ce contexte, il est intéressant de noter le pourcentage d'aide obtenu par les entreprises innovantes ces dernières années pour leurs activités de R\&D. Le graphique 4 montre que seule une petite partie des entreprises industrielles en général, et des PME en particulier, a bénéficié d'une aide publique. Si l'on inclut tous les financeurs, ce taux est deux fois plus élevé dans les grandes entreprises que dans les moyennes, et plus de deux fois plus élevé que dans les petites entreprises.

\section{Graphique 4 : Part des entreprises innovantes ayant bénéficié d'un financement public}

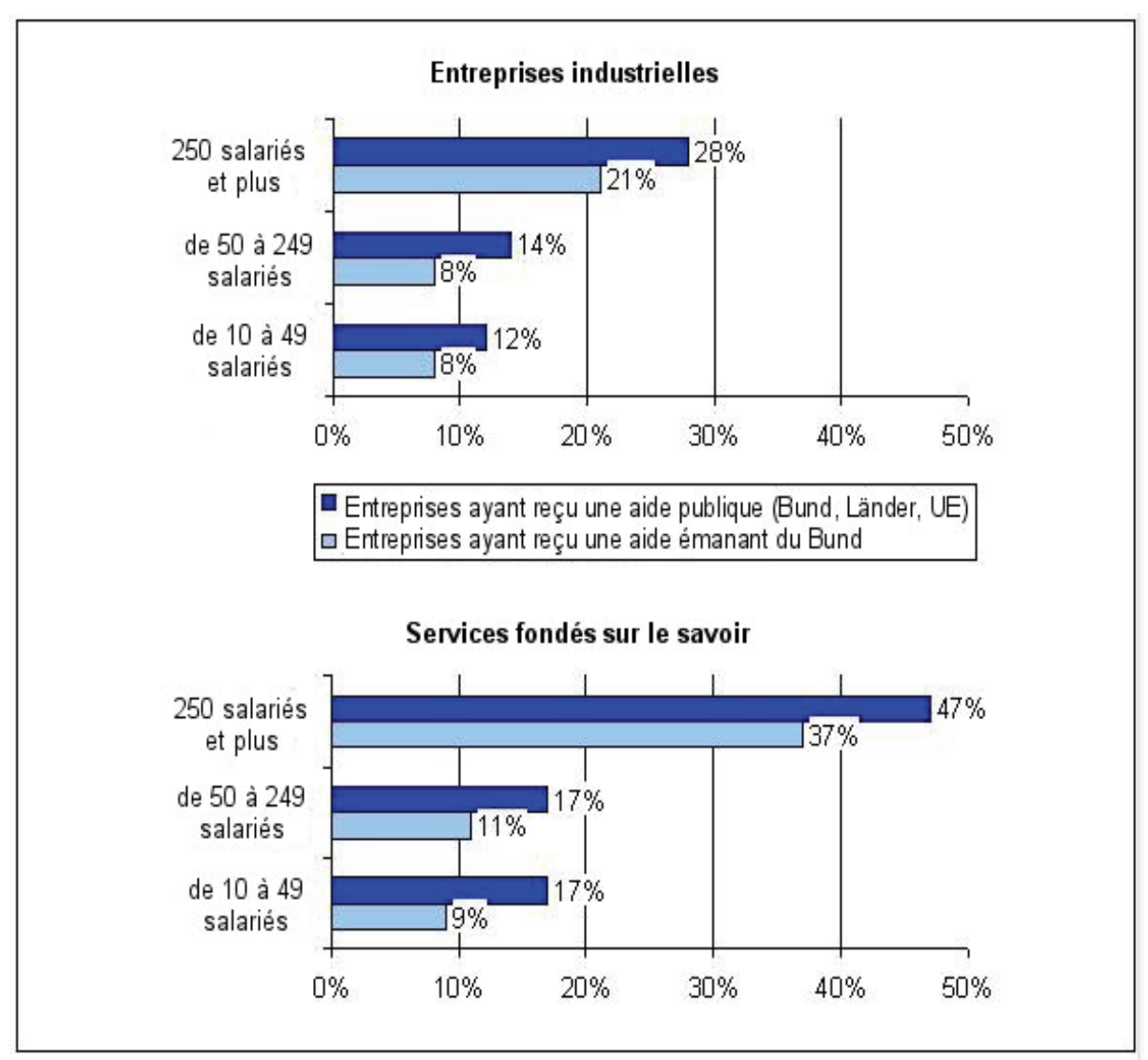

Source : enquête sur l'innovation du ZEW 2005, sur la base de normes CIS4 ${ }^{10}$.

\footnotetext{
${ }^{10}$ Source : http://www.zew.de/de/publikationen/innovationserhebungen/euroinno.php3 [consulté le 06/09/10].
} 
Les entreprises de services fondées sur le savoir - en particulier les grandes ( 250 salariés et plus) - bénéficient nettement plus des aides publiques que les entreprises industrielles : au cours de la période prise en considération, près de la moitié d'entre elles a reçu des aides émanant du Bund, des Länder ou de l'UE. Parmi les PME, les taux ne sont que de $17 \%$ environ. Le graphique souligne aussi que, pour toutes les catégories d'entreprises, l'Etat fédéral est le principal financeur.

\subsection{Domaines cibles et dispositifs de financement}

En matière d'aide aux PME innovantes, plusieurs dispositifs de soutien existent pour les différents domaines, comme on le voit sur le tableau 2. On peut également observer qu'il existe en Allemagne de nombreux programmes de soutien favorisant indirectement les PME.

Ainsi, afin de renforcer la coopération entre science et PME, les universités et les organismes de recherche extra-universitaires bénéficient d'une prime de recherche s'ils réalisent des travaux de R\&D pour le compte de PME ou en collaboration avec elles. Ce dispositif incitatif vise à instaurer une meilleure exploitation des savoirs et des résultats de la recherche dont les PME bénéficient puisque la prime de recherche fait d'elles des partenaires plus attractifs.

\subsection{Deux exemples de programmes de soutien à l'innovation pour les PME}

\subsubsection{PRO INNO II : programme de subventions pour les PME}

La mesure PRO INNO II « Soutenir l'accroissement de la capacité d'innovation des PME », une initiative du ministère fédéral de l'Economie et de la Technologie (BMWi), poursuit la tradition de promotion des coopérations de R\&D initiée au début des années 1990. Les subventions sont allouées pour les projets de recherche réalisés à travers différentes formes de coopérations entre des PME et leurs partenaires, ainsi que pour la création d'activités de R\&D. Ces aides sont versées aux PME et à leurs partenaires de recherche. Sont possibles les formes de projets suivantes :

- projets de coopération entre PME ;

- projets de coopération entre PME et organismes de recherche ;

- projets de coopération d'une PME avec une commande de R\&D à un organisme de recherche;

- échange de personnel entre PME et organisme de recherche ;

- projets de lancement pour les PME menant pour la première fois des activités de R\&D ;

- depuis peu, également prestations de service et de conseil soutenant l'innovation. 
Tableau 2 : Domaines cibles et instruments de soutien

\begin{tabular}{|c|c|c|c|c|c|}
\hline & Subventions & $\begin{array}{c}\text { Capital- } \\
\text { investissement }\end{array}$ & $\begin{array}{c}\text { Prêts } \\
\text { pour } \\
\text { la } \\
\text { R\&D }\end{array}$ & $\begin{array}{c}\text { Cautions + } \\
\text { Garanties }\end{array}$ & Conseil \\
\hline \begin{tabular}{|l|} 
Production \\
d'innovation : \\
produits, \\
procédés, services \\
nouveaux ou \\
améliorés
\end{tabular} & $\bullet$ & - & $\bullet$ & & $(\bullet)$ \\
\hline \begin{tabular}{|l|} 
Coopération \\
entre science et \\
PME : \\
exploitation de \\
savoirs et de \\
résultats de la \\
recherche \\
\end{tabular} & $\bullet(\bullet)$ & & & & \\
\hline \begin{tabular}{|l|} 
Créations et \\
PME primo- \\
innovantes : \\
augmentation du \\
nombre de PME \\
menant des \\
activités de $R \& D$ \\
\end{tabular} & $\bullet(\bullet)$ & $\bullet$ & & & $(\bullet)$ \\
\hline \begin{tabular}{|l} 
Accroissement \\
quantitatif et \\
qualitatif des \\
activités de R\&D \\
dans les PME : \\
augmentation de \\
la qualité dans les \\
PME existantes \\
\end{tabular} & - & - & • & & - \\
\hline \begin{tabular}{|l|} 
Mise en réseau \\
avec des acteurs \\
de l'innovation \\
de l'ensemble de \\
la chaîne de \\
valeur : \\
réseaux \\
d'innovation \\
régionaux et \\
sectoriels \\
\end{tabular} & $(\bullet)$ & & & & \\
\hline
\end{tabular}




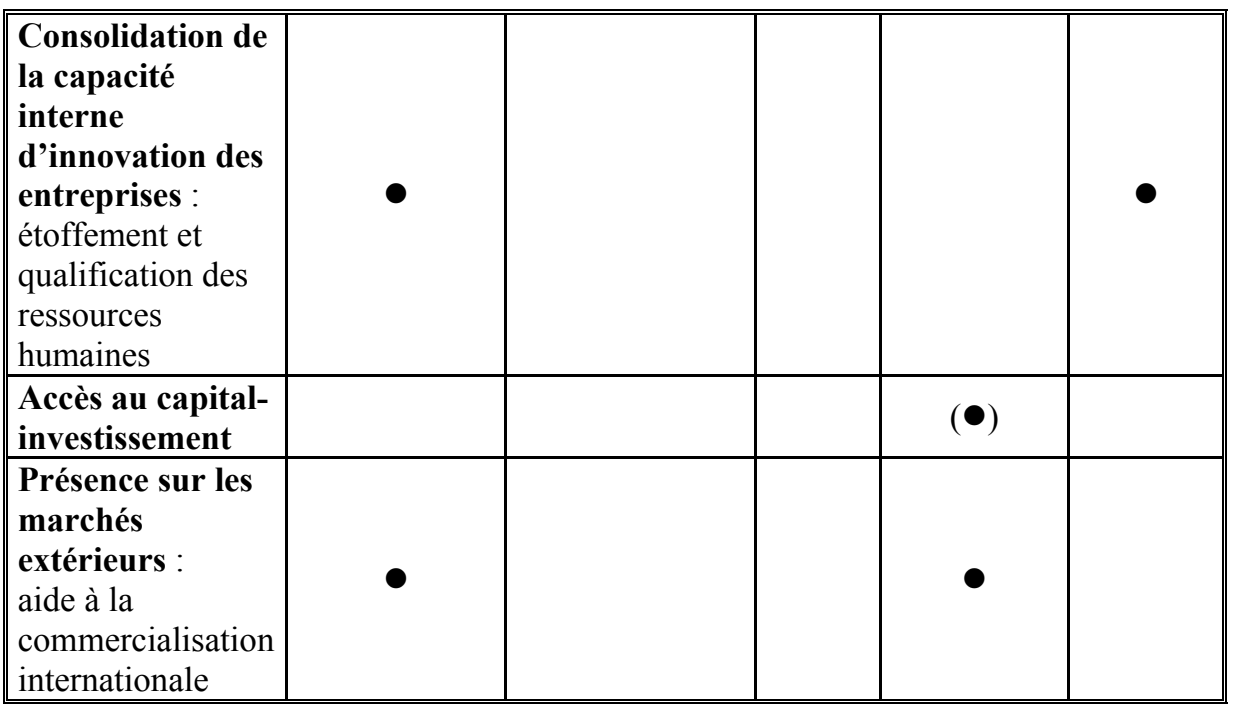

Légende : • Aides versées directement aux PME $(\bullet)$ Aides versées à d'autres structures pour des prestations réalisées pour des PME.

Source : présentation de l'auteur.

Les coopérations ont pour but le développement de produits, procédés ou services techniques innovants, l'une des forces du programme de soutien aux PME étant de ne pas se limiter à certaines technologies ; par ailleurs la procédure de demande est relativement simple. Ce dispositif comprend des subventions pouvant aller jusqu'à $350000 €$ avec des taux de financement de 40 à $50 \%$ selon la taille et la localisation de la PME. En 2007, ce programme a représenté un volume total d'environ 180 millions $€$ d'aides allouées aux PME et à leurs partenaires de recherche. Une évaluation des programmes de financement PRO INNO I et PRO INNO II, réalisée par l'Institut Fraunhofer de recherche sur l'innovation et les systèmes ${ }^{11}$ et achevée en 2006, a confirmé le haut niveau d'acceptation au sein du groupe cible et la capacité d'adaptation du programme aux contraintes des PME.

\subsubsection{Programme d'innovation ERP, programme de crédit et d'investissement}

Le programme d'innovation ERP, proposé par la banque KfW, a pour objectif le financement à long terme (1) d'activités de recherche et développement de nouveaux produits, procédés ou services (2) et de leur lancement sur le marché. Dans ce dernier domaine, les possibilités de financement public sont rares dans la mesure où la réglementation européenne en matière d'aides d'Etat imposait jusque-là des règles contraignantes. Le programme d'innovation ERP comporte

${ }^{11}$ Voir : Kulicke et al. (2005), Lo et al. (2006), Kulicke et al. (2006). 
une formule crédit et une formule investissement. Dans le premier cas, le financement se fait sous la forme d'un prêt classique combiné à un prêt subordonné. La formule investissement favorise indirectement les PME car les investisseurs sont soutenus au moyen d'un crédit à taux avantageux pour investir dans une PME pour le financement de (1) ou (2).

Le programme d'innovation ERP, dans la formule crédit en phase de $R \& D$, octroie des crédits à des entreprises et à des indépendants, en particulier à des PME. Tous les coûts jusqu'à la clôture des travaux de développement préalables à l'exploitation commerciale peuvent être couverts. Le prêt peut s'élever à cinq millions $€$. Si les moyens nécessaires au financement de la phase de lancement sur le marché concernent des entreprises et des indépendants qui sont des PME, les investissements de commercialisation de nouveaux produits et procédés de production et ceux relatifs à certaines mesures de lancement sur le marché peuvent être couverts jusqu'à hauteur de $50 \%$ (anciens Länder) ou $80 \%$ (nouveaux Länder, Berlin). Le crédit maximum est de 1 à 2,5 millions $€$. En 2006, dans le cadre de la formule crédit du programme d'innovation ERP, la KfW a accordé plus de 1,66 milliard $€$ aux PME (500 salariés max.).

\section{Synthèse et évaluation de la politique de soutien au Mittelstand}

Pour résumer, on peut retenir les points suivants :

- depuis plusieurs années, on observe en Allemagne une stagnation, voire dans certains cas un recul du nombre de PME innovantes menant des activités de R\&D. L'industrie manufacturière et les secteurs des services high-tech regroupent une grande partie des PME n'ayant pas d'activités de R\&D ;

- l'Allemagne est depuis longtemps dotée d'un dispositif de soutien diversifié pour les PME, avec des offres du Bund, de la banque KfW et des Länder. Le soutien au Mittelstand a une place cruciale dans la politique économique et de l'innovation. Les offres de financement sont si nombreuses que les PME déplorent souvent le manque de lisibilité et la complexité des procédures de demande ;

- on peut globalement qualifier le soutien de fortement sélectif dans la mesure où seule une petite partie des PME bénéficie d'un financement public ou est éligible à ces dispositifs. Il n'y a pas d'incitation fiscale à la R\&D à une large échelle, mais il est actuellement envisagé d'introduire de tels instruments ;

- le soutien aux PME innovantes met clairement l'accent sur la promotion de l'innovation (R\&D orientée marché) avec des mesures visant à amener davantage de PME à investir dans la recherche $(R \& D$ très en amont du marché);

- parmi les dépenses d'innovation, priorité est donnée aux dépenses de R\&D ; à l'heure actuelle, rares sont les possibilités de financer les dépenses de 
lancement sur le marché, même si cela s'avère souvent, en particulier pour les PME, un point faible en matière de valorisation des résultats de la recherche;

- le soutien aux PME innovantes ne se limite pas à des domaines technologiques précis (ouverture thématique), afin de tenir compte de la grande hétérogénéité et du large spectre d'activités de cette catégorie d'entreprises ;

- après un net recul ces dernières années, davantage de moyens sont de nouveau déployés depuis 2006 pour le soutien à l'innovation. Cela vaut pour le Bund comme pour la plupart des Länder;

- le soutien aux PME met très fortement l'accent sur l'idée de coopération, qui domine le plus grand programme d'innovation PRO INNO II. A l'heure actuelle, les programmes de subvention de la $R \& D$ soutiennent exclusivement les partenariats ;

- en matière de soutien aux PME innovantes précisément, il est fait appel à divers instruments de financement: il s'agit souvent de subventions non remboursables, parfois aussi de prêts et de fonds de capital-investissement ;

- ces dix dernières années, le renforcement des infrastructures de soutien à l'innovation s'intensifie. En font partie de nombreuses mesures de mise en réseau des PME avec d'autres entreprises, avec des universités et des organismes de recherche, en particulier dans les nouveaux Länder. Dans ce cas, les aides ne sont pas versées aux PME elles-mêmes, mais aux structures de gestion de réseau, aux organismes de conseil, etc.;

- le soutien au Mittelstand a un poids politique important, notamment dans le cadre de la stratégie «Hautes Technologies» de l'Etat fédéral, mais les principaux axes de financement de cette stratégie sont clairement la consolidation des universités, des organismes de recherche extrauniversitaires et le renforcement de grandes entreprises très actives en matière de R\&D ;

- de nouveaux instruments de promotion, comme le concours de promotion des clusters d'excellence, mettent l'accent sur la formation des élites ou vont, comme la prime de recherche, dans le sens du renforcement des infrastructures de recherche. Les PME ne bénéficient que partiellement de ces dispositifs.

DEPUIS MI-2008, LA PLUPART DES OFFRES DÉDIÉES AUX PME INNOVANTES sont progressivement regroupées dans le nouveau dispositif « Programme central d'innovation pour le Mittelstand (ZIM) ». Cette évolution implique de poursuivre de manière intégrée les éléments qui caractérisaient déjà l'aide aux PME ces dernières années : coopération, mise en réseau, conseil.

Traduction de Marie GRAVEY 


\section{Indications bibliographiques}

BUNDESMINISTERIUM FÜR BILDUNG UND FORSCHUNG (BMBF), Die Hightech-Strategie für Deutschland, BMBF, Bonn/Berlin, 2006

BUNDESMINISTERIUM FÜR WIRTSCHAFT UND TECHNOLOGIE (BMWI), «Der Mittelstand in der Bundesrepublik Deutschland: Eine volkswirtschaftliche Bestandsaufnahme», Dokumentation, $\mathrm{n}^{\circ}$ 561, février 2007, p. 1-64

BUNDESMINISTERIUM FÜR WIRTSCHAFT UND TECHNOLOGIE (BMWI), Schlaglichter der Wirtschaftspolitik. Monatsbericht, février 2008, BMWi, Berlin

COMMUNAUtÉS EUROPÉENNES, Die neue KMU-Definition. Benutzerhandbuch und Mustererklärung [en ligne], Commission européenne, Bruxelles, 2006 :

http://europa.eu.int/comm/enterprise/enterprise_policy/sme_definition/index_de.htm [consulté le 07/01/10]

KLÖS H.-P., Forschungsförderung in Deutschland: Stimmen Angebots- und Nachfragebedingungen für den Mittelstand?, Gutachten für die Stiftung Industrieforschung [en ligne], Institut der deutschen Wirtschaft, Cologne, 2007 : http://www.argez.de/library/documents/Klös_32609.pdf [consulté le 07/01/10]

Koschatzky K., Reinhard M., Grenzmann C., Forschungs- und Entwicklungsdienstleistungen in Deutschland. Struktur und Perspektiven eines Wachstumsmarktes, Fraunhofer IRB Verlag, Stuttgart, 2003

KULICKE M., BÜHRER S., LO V., Untersuchung der Wirksamkeit von PRO INNO - PROgramm INNOvationskompetenz mittelständischer Unternehmen - Modul 1: Einschätzung der Ergebnisse des Programms PRO INNO, Fraunhofer IRB Verlag, Stuttgart, 2005

Kulicke, M.; BÜHRER, S.; RuHLAND, S., PRO INNO II - PROgramm zur Förderung der Erhöhung der INNOvationskompetenz mittelständischer Unternehmen - Entwicklung des Programmanlaufs von August 2004 bis Ende 2005, Fraunhofer IRB Verlag, Stuttgart, 2006

LO V., KULICKE M., KIRNER E., Untersuchung der Wirksamkeit von PRO INNO - PROgramm INNOvationskompetenz mittelständischer Unternehmen - Modul 2: Analyse von in den Jahren 2001/2002 abgeschlossenen FuE-Kooperationsprojekten, Fraunhofer IRB Verlag, Stuttgart, 2006

LO V., WOlF B., KOSCHATZKY K., WeIß D., Förderung von Forschung und Entwicklung bei Wachstumsträgern in benachteiligten Regionen - Evaluation des BMWi-Programms INNO-WATT, Fraunhofer IRB Verlag, Stuttgart, 2006

RAMMER C., «Innovationspotenzial von KMU nutzen», ZEWnews [en ligne], 11/2005, novembre 2005, p. 12 : ftp://ftp.zew.de/pub/zew-docs/zn/zn1105.pdf [consulté le 07/01/10]

RAMMER C., «Wenig Innovationsdynamik», Sonderteil ZEWnews [en ligne], Sonderteil 03/2007, mars 2007, p. 1-4: ftp://ftp.zew.de/pub/zew-docs/zn/schwerpunkte/innovation Innovationen0307.pdf [consulté le $07 / 01 / 10]$

Rammer C., Zimmermann V., Müller E., Heger D., AschHoff B., Reize R., Innovationspotenziale von kleinen und mittleren Unternehmen, Nomos, Baden-Baden, 2006

STIFTERVERBAND FÜR DIE DEUTSCHE WISSENSCHAFT, Forschung und Entwicklung in der Wirtschaft, Stifterverband für die deutsche Wissenschaft, Essen, 2006

STIFTERVERBAND FÜR DIE DEUTSCHE WISSENSCHAFT, FuE-Datenreport 2007. Tabellen und Daten. Forschung und Entwicklung in der Wirtschaft. Bericht über die FuE-Erhebungen 2005 [en ligne], Stifterverband für die deutsche Wissenschaft, Essen, 2007: http://www.stifterverband.de/pdf/ fue_datenreport_2007.pdf[consulté le 07/01/10]. 



\section{Le rôle des PME dans les pôles de compétitivité français}

\section{Emmanuel LEPRINCE}

Comment soutenir l'activité des PME innovantes en France ? L'une des solutions consiste à faciliter leur accès au marché en renforçant leurs relations avec les grandes entreprises, publiques et privées. C'est cet objectif que poursuit, depuis son lancement en 2006, le Pacte PME, appuyé par les pouvoirs publics et mis en œuvre par le Comité Richelieu, avec le soutien financier et opérationnel d'OSEO ainsi que la participation active d'un réseau de 40 partenaires et 21 pôles de compétitivité. Emmanuel Leprince, ancien délégué général du Comité Richelieu et aujourd'hui délégué général de l'association Pacte PME, présente ici les enjeux du programme auquel ont participé près de 2500 PME françaises au cours de la période 2006-2008.

Créé en 1989, le Comité Richelieu est une association exclusivement composée de PME innovantes, indépendante des pouvoirs publics. Elle regroupe 250 entreprises qui présentent le profil moyen suivant: un effectif de 55 salariés; 6,7 millions $€$ de chiffre d'affaires dont $31,5 \%$ réalisés à l'exportation et $33,2 \%$ réinvestis en $\mathrm{R} \& \mathrm{D}$.

\section{Le Pacte PME}

Opéré conjointement par le Comité Richelieu et OSEO, l'agence publique française des PME, le Pacte PME est une forme de Small Business Act à la française. Ce dispositif américain, âgé d'un peu plus de cinquante ans et que la crise financière actuelle replace sur le devant de la scène, oriente vers les PME américaines $23 \%$ des marchés publics. Cela représente chaque année une manne de 100 milliards \$ de contrats pour les PME américaines... Alors que les PME françaises ne se voient attribuer que $12 \%$ des marchés publics hexagonaux, qui pèsent 100 milliards $€$ de contrats annuels dont les $88 \%$ restants vont aux grandes entreprises.

C'est ce sujet d'accès à la commande et au chiffre d'affaires, mis en évidence dans le Livre blanc publié par le Comité Richelieu en 2003, qui est à l'origine du Pacte PME. Son importance est encore accrue dans le contexte de crise que nous traversons car les PME doivent faire face, non seulement à un problème de credit crunch, mais aussi au phénomène de contract crunch, c'est-à-dire des reports de contrats qui menacent leur chiffre d'affaires. Pour ce faisceau de raisons, il est pertinent de se poser la question de savoir s'il faut ou non orienter une partie des 1500 milliards $€$ d'achats publics européens vers les PME - et si oui, comment le faire - afin de permettre à ces entreprises de réaliser un chiffre d'affaires suffisant pour leur permettre de traverser la crise. 
A la différence du Small Business Act américain, le Pacte PME n'est pas contraignant et n'impose pas de quotas. C'est un dispositif incitatif qui s'appuie sur le volontariat de ses signataires, grandes entreprises privées ou grands acheteurs publics. En signant le document qui témoigne de leur adhésion à la dynamique du Pacte PME, les grands comptes s'engagent à la transparence sur la part des PME dans leurs achats en publiant des indicateurs, ainsi qu'à mettre en place des outils pour intensifier et améliorer leurs relations avec les PME innovantes susceptibles de leur proposer de nouveaux produits et services. La particularité de cette démarche est de s'effectuer dans l'intérêt mutuel des deux parties. Celui des PME bien sûr, mais aussi celui des grands comptes. Ces derniers, en signant le Pacte PME reconnaissent qu'ils n'ont pas un accès suffisant aux PME porteuses d'innovations qui représentent pour eux un gain de compétitivité potentiel. Deux raisons sont à l'origine de cette situation. D'une part, il n'est pas aisé pour ces grands comptes d'identifier les PME susceptibles de répondre avec pertinence à leurs besoins. D'autre part, leurs acheteurs ne veulent pas prendre de risques et préfèrent travailler avec des entreprises établies. Pour réussir une innovation ouverte, les dirigeants de ces grandes entreprises doivent trouver les mécanismes qui leur permettront de travailler avec ce vivier d'idées nouvelles et le Pacte PME leur propose justement les outils adéquats.

Les résultats sont au rendez-vous : l'ensemble des achats réalisés auprès des PME par les 50 grands comptes signataires est aujourd'hui de près de 40 milliards $€$. Trois facteurs concourent à l'efficacité du Pacte PME. Tout d'abord, le caractère collectif de la démarche: des échanges d'informations afin de déterminer les meilleures pratiques sont organisés entre les grands comptes. Ensuite, il est piloté par le Comité Richelieu, dont l'une des vocations consiste à recueillir le retour d'expérience des PME innovantes sur les relations qu'elles entretiennent avec leurs grands donneurs d'ordres. Enfin, il reçoit un soutien opérationnel et financier des pouvoirs publics, notamment grâce à OSEO. Il est aussi appuyé, au moins de manière informelle, par l'exécutif français. Tout en insistant sur la valeur que prend à ses yeux sa philosophie résidant dans le volontariat, le président de la République qualifie le Pacte PME de "programme clé pour l'économie française » dans la préface qu'il a rédigée pour son rapport d'activité 2007.

Les grandes entreprises qui participent au Pacte PME sont, pour la plupart, installées en France. Certaines sont totalement privées, comme Total, Schlumberger et Ericsson France. Mais on y trouve aussi des ministères - entre autres le ministère de la Défense et le ministère de l'Intérieur - et de grandes entreprises publiques comme EDF ou la SNCF dont le volume d'achats, bien que très élevé, ne revient que pour une faible part aux PME innovantes. Enfin trois collectivités locales : la première à s'engager a été la Ville de Paris, dont le volume annuel d'achats aux entreprises s'élève à 1,2 milliard $€$, précédant Angers Loire Métropole et la Ville de Versailles.

Près de 2500 PME françaises se sont inscrites au programme entre 2006 et 2008. Ce sont majoritairement des entreprises qui ont dépassé la phase start-up : 
en moyenne, elles existent depuis cinq à six ans et emploient 55 salariés. Mais, malgré ce profil type, elles ont du mal à se développer et il s'agit là d'une réalité commune à la France, à l'Allemagne, voire à l'Europe en général. Certes, il existe de grandes différences entre la France et l'Allemagne. Cependant, une comparaison des tissus économiques en Europe et aux Etats-Unis fait apparaître des écarts encore plus importants. Par exemple, dans la liste des 500 principaux acteurs mondiaux, on compte 50 grandes entreprises que l'on peut qualifier de « jeunes », c'est-à-dire créées après 1975. 26 d'entre elles sont américaines et seulement 3 européennes, dont SAP qui est allemande. Ces chiffres mettent en lumière le problème du renouvellement de la population des très grandes entreprises, qui est un élément critique en termes de croissance. C'est pourquoi le programme essaie avant tout de soutenir les entreprises qui ont le potentiel de devenir des acteurs mondiaux.

Afin d'identifier dans tous les secteurs ces PME prometteuses, qui sont aussi recherchées pour leur potentiel d'innovation, nous avons mis en place un réseau de partenaires, qui couvre tout le territoire français métropolitain, et des échanges d'information descendants et ascendants. Très concrètement, il s'agit d'organiser pour chaque grande entreprise une conférence annuelle réunissant $120 \mathrm{PME}$ en moyenne. Au cours de cette rencontre, la grande entreprise présente sa politique d'achats, détaillant ses méthodes d'acquisition et ses thèmes d'intérêt technologique. La remontée d'informations s'effectue à l'occasion de présentations organisées, à un rythme hebdomadaire, sur des thèmes technologiques précis, par exemple la « voix sur IP » (Voice on IP). Chaque PME dispose de cinq minutes pour présenter sa technologie et la valeur ajoutée de celle-ci à un auditoire composé d'acheteurs et de responsables de l'innovation des grands groupes. Ces événements, que nous appelons « rencontres [met]» reprennent la logique des présentations aux investisseurs qui avaient cours voici quelques années. Mais ce qui est en jeu dans le Pacte PME, ce n'est plus le financement des projets, c'est une mise en contact susceptible d'aboutir à la conclusion d'un contrat. Nous en avons dénombré 78 en 2006 et, en 2007, ce ne sont pas moins de 306 contrats entre PME et grands groupes qui ont été initiés par ces rencontres. De plus, des offres de PME répertoriées sous 60 catégories différentes sont présentées en ligne sur un site Internet bilingue français-anglais. Par exemple, aujourd'hui même, nous avons ouvert un thème intitulé paperless (sans papier). Notre équipe, composée de 12 personnes, a identifié une vingtaine de PME françaises dont les offres sont très pointues sur ce sujet. Notre connaissance des besoins des grands comptes nous permet d'informer rapidement ceux qui sont concernés. Dans le domaine de l'innovation, il est important que les acteurs se parlent et se voient car, lorsque les acteurs ne sont pas informés, les grandes entreprises ont tendance à se tourner vers leurs fournisseurs habituels, en excluant ainsi les nouveaux entrants. Mais ce sont autant de processus qui prennent du temps. Les modes de mise en relation proposés par le Pacte PME - conférences, rencontres [met], bottom-up en ligne - 
sont déterminants car ils permettent de favoriser ces échanges en réalisant un gain de temps précieux.

Pourtant, il ne suffit pas de mettre en contact les PME et les grands groupes : beaucoup d'obstacles se lèvent avant la signature des contrats. Un exemple? Celui du coût que représente pour une grande entreprise la phase de test d'un produit provenant d'une PME, ou encore son adaptation à un besoin précis. Très souvent, le produit proposé par une PME innovante ne répond pas exactement au besoin du grand groupe qui est pourtant intéressé. Le travail qui demeure à effectuer doit être financé. Mais par qui ? En général, c'est la PME qui doit prendre en charge le financement, ce qui pose problème. Pour le résoudre, nous nous sommes inspirés d'un dispositif norvégien, mis au point par Innovation Norway. Cette agence d'innovation norvégienne, avant d'octroyer ses aides aux PME, leur demande de se présenter avec un client potentiel. Celui-ci, pour prouver qu'il est intéressé, doit accepter de financer un tiers du projet. L'Etat fournit le deuxième tiers sous forme de subvention et la PME apporte, quant à elle, le dernier tiers du montant du projet. A l'issue des travaux, c'est la PME qui conservera la propriété intellectuelle des résultats, tandis que le grand groupe aura un droit de premier regard sur les travaux pendant six mois, voire un an. En Norvège, une centaine de projets sont montés chaque année avec un budget de 38 millions $€$ par an. En France, ce système adopté en 2008 a permis de financer une quinzaine de projets. Nous développons actuellement ce programme, financé par OSEO, l'Agence française de l'innovation. Ainsi, les pouvoirs publics français, après avoir alloué des financements pour aider les banques et les investisseurs à aller vers les PME, reconnaissent qu'il faut aussi inciter les grands groupes à aller dans ce sens. Nous avons également mis en place une garantie financière qui permet à la PME d'apporter une sécurité à l'acheteur. Si la PME rencontre des difficultés pendant l'exécution du contrat, l'acheteur sera remboursé de la somme qu'il a avancée.

Nous organisons aussi des groupes de travail réunissant les grands comptes afin de stimuler l'échange de bonnes pratiques pour optimiser la collaboration avec les PME innovantes. Un exemple parmi ces bonnes pratiques : la mise en place d'une équipe d'évaluation dédiée, dont l'existence est encore rare au sein des grandes entreprises. En règle générale, ce sont les services Achats ou R\&D qui reçoivent les PME innovantes venues proposer leurs produits et services. Or, les responsables de la R\&D des grands groupes - ceux-là même qui, souvent, collaborent aux projets des pôles de compétitivité - peuvent se montrer réticents à travailler avec les PME innovantes, qui pourraient les concurrencer. En revanche, il arrive que les responsables des services «métiers" souhaitent travailler avec des entreprises qui apportent des idées nouvelles. Et lorsque deux solutions leur sont proposées, l'une en interne par la R\&D et l'autre en externe par la PME, ce sont eux qui devront faire un choix. Un grand groupe doit avoir des équipes d'évaluation dédiées aux idées extérieures, comités à la fois déconnectés de la $R \& D$ mais aussi des services achats, qui ont eux tendance à vouloir travailler avec des entreprises de plus de dix ans et dont le chiffre d'affaires est supérieur à 
100 millions $€$. Pour permettre l'innovation ouverte au sein des grands comptes, il faut appliquer des règles différentes pour les PME. Ceux qui essaient de mettre en place ce genre d'équipes dédiées sont encore minoritaires. Nous essayons d'avancer avec eux sur le sujet des bonnes pratiques.

La motivation du personnel dans les grandes entreprises est un deuxième exemple de bonne pratique. Afin de motiver leurs acheteurs à aller chercher les idées nouvelles au dehors, certains grands comptes leur proposent une prime annuelle proportionnelle à la part de nouveaux fournisseurs dans leurs achats. En guise de dernier exemple, citons le renforcement des fournisseurs stratégiques. L'une des raisons pour lesquelles les grandes entreprises n'ont pas envie de travailler avec les PME innovantes, c'est leur fragilité. Elles redoutent ce qui se produirait si la PME venait à fermer, alors que la signature d'un contrat aurait fait d'elle un fournisseur clé. Nous mettons donc en place des programmes, appuyés sur des dispositifs financiers, pour renforcer les fournisseurs stratégiques des grandes entreprises. Dans le même objectif, nous aidons les PME à trouver du chiffre d'affaires à l'exportation en favorisant leur portage à l'international par les grands comptes, dans un état d'esprit d'intérêt partagé.

Pour chaque signataire, nous organisons des commissions rassemblant les PME qui travaillent avec lui, afin d'être en mesure de lui remonter un retour d'expérience de ces entreprises. Ces retours d'expérience sont construits sur la base d'enquêtes qui permettent de mieux cerner le comportement du grand groupe avec les PME. Ces informations qualitatives sont présentées de manière confidentielle à chaque grand groupe. Le rapport d'activité publié annuellement dédie, à chaque grand compte qui a signé le Pacte PME, une fiche sur laquelle figurent des indicateurs quantitatifs sur la part des PME dans ses achats. De 2007 à 2008, la moyenne de cette part chez l'ensemble des signataires est passée de $18 \%$ à plus de $19 \%$, ce qui représente une augmentation de 900 millions $€$.

\section{La participation des PME aux pôles de compétitivité}

En France, les PME attendent beaucoup des pôles de compétitivité. Nombre d'entre elles les ont intégrés afin d'y rencontrer de grandes entreprises, dans l'espoir d'en devenir fournisseur. Mais ceci se produit encore assez peu et engendre davantage de concurrence que de partenariats puisque, comme nous l'avons vu précédemment, les représentants des grandes entreprises dans les pôles de compétitivité français ne sont pas des responsables Achats, mais R\&D qui tentent avant tout de faire financer leurs travaux.

Le Pacte PME a deux modes de relation avec les pôles de compétitivité. En premier lieu, nous avons appliqué aux 20 pôles de compétitivités signataires en 2008, qui sont les 20 principaux pôles français, une même logique de transparence qui suit, non pas le volume d'achat mais, cette fois-ci, celui des soutiens publics à la $\mathrm{R} \& \mathrm{D}$. Les pôles permettent de financer des projets de coopération technologique. Il semble qu'en Allemagne, les pôles gèrent eux-mêmes les finan- 
cements qu'ils reçoivent. En France, les pôles ne gèrent pas d'enveloppe financière eux-mêmes et doivent, à chaque fois, transmettre à de multiples financeurs les projets provenant des entreprises. Or, nous avons constaté que la part financière des PME dans ces projets de coopération technologique était assez faible au regard de celle des grandes entreprises, plus de $80 \%$, à laquelle s'ajoute encore celle des laboratoires. Ce déséquilibre est certainement lié à la gouvernance des pôles, dans laquelle l'Etat français n'a pas souhaité intervenir et qui est donc plutôt portée par les entreprises les plus importantes, ce qui bloque le système puisque c'est la gouvernance qui labellise les projets.

Nous avons proposé aux pôles de compétitivité d'adopter la démarche du Pacte PME pour contribuer à renforcer la participation des PME, notamment en donnant une visibilité à la part qu'elles occupent dans les budgets de coopération technologique. Le rapport d'activité comprend également une fiche par pôle, indiquant le budget total annuel de chacun. C'est ainsi que nous avons pu constater la relative faiblesse de la part des PME. En 2007, elles n'ont bénéficié que d'un peu moins de 40 millions $€$ en provenance du Fonds Unique Interministériel (FUI), qui dépend du ministère des Finances, de l'Economie et de l'Emploi. C'est peu comparé aux autres sources de financement des PME que sont OSEO Innovation (financement direct) et le Crédit d'Impôt Recherche (dispositif fiscal).

En deuxième lieu, le Pacte PME peut faciliter l'accès au marché et aux utilisateurs des résultats des projets financés dans le cadre des pôles, particulièrement ceux qui sont portés par les PME. Comment? Ils figurent parmi les offres de la place de marché en ligne du Pacte PME, afin qu'ils puissent trouver des débouchés auprès des services Achats des 50 grands groupes signataires. En outre, sur la base de leur domaine d'application, nous construisons des échanges thématiques entre PME et grands groupes.

LES PÔLES DE COMPÉTITIVITÉ sont clairement devenus des dispositifs structurants du territoire français. Il n'en demeure pas moins que le rôle qu'ils jouent dans l'écosystème des PME reste encore à affirmer. Le Pacte PME se penche également sur ce point en enquêtant sur l'image que les PME ont des pôles auxquels elles appartiennent. Nous leur avons demandé si elles estimaient que leur participation était bien prise en compte. Les résultats que nous avons publiés dans notre rapport d'activité 2007 montrent qu'à l'époque, sur les 20 pôles avec lesquels nous travaillions, seuls quatre obtenaient le satisfecit de plus de la moitié de leurs PME membres. Il nous faut collaborer d'autant plus profondément avec les 16 autres. Afin de les aider à progresser dans cette voie, nous déclinons pour les pôles les groupes de travail organisés pour les grands comptes sur les bonnes pratiques comme, par exemple, une évolution de la gouvernance où des associations locales représenteraient l'ensemble des PME. 


\title{
Le capital-risque pour les petites et moyennes entreprises (PME) innovantes
}

\author{
Michael GROß
}

Le recours au capital-risque pour le financement de PME constitue un soutien considérable à la création d'une génération d'entreprises innovantes et compétitives à l'échelle internationale. Fort de ce constat, Michael Groß, co-dirigeant jusqu'en 2010 de BC Brandenburg Capital $\mathrm{GmbH}$, aborde dans sa contribution l'évolution historique et la situation actuelle du financement par capital-risque pour les PME en Allemagne, en regard du cas de la France et de la Grande-Bretagne. II précise ensuite le rôle de BC Brandenburg Capital GmbH comme modèle de partenariat public-privé à l'échelle du Brandebourg pour identifier enfin les acteurs du processus de financement des PME.

\section{Introduction}

Les grandes lignes de l'évolution historique du financement par capital-risque en Allemagne

Selon la conception de la fédération allemande des sociétés de capital-risque (BVKBundesverband Deutscher Kapitalbeteiligungsgesellschaften-German Private Equity and Venture Capital Association e.V.), à laquelle l'auteur se rattache, l'évolution historique du capital-risque peut se diviser en quatre périodes :

Phase 1 «Léthargie »: La première phase correspond plus ou moins à la période allant des années 1980 au début des années 1990. Du côté de l'offre, on trouve un petit nombre de sociétés d'investissements, dotées d'un faible volume de capital et ne réalisant pas activement de levée de fonds. Comme il n'y a alors pas encore de marché établi pour les investissements ou participations, les sociétés d'investissements se trouvent face à un nombre limité d'opportunités d'investissements. Au cours de cette phase, les entreprises financent le plus souvent leurs investissements de manière traditionnelle, en faisant appel à leur banque ou en utilisant leurs propres excédents. Les structures à la recherche de capitaux sont alors critiques à l'égard des fournisseurs de capital-risque (CR) : l'acceptation n'est pas encore là. Il n'y a pas encore de tendances sectorielles, comme celles qui apparaitront au cours de la deuxième phase.

L'environnement (peu de places boursières, peu d'incitations publiques et des possibilités de sortie limitées) est tout sauf incitatif.

Phase 2 «Boom»: Au cours de la phase de boom, qui a commencé autour de 1992 et a pris fin à l'éclatement de la bulle Internet en mars 2000, l'offre de capital-investissement augmente. Les sociétés sont de plus en plus nombreuses, et le volume de capital s'accroît considérablement. Certaines sociétés de capitalinvestissement commencent à lever des fonds. 
C'est au cours de la deuxième phase que se forge la notion de «nouvelle économie ». La multiplication des ordinateurs et l'apparition de nouveaux moyens de communication entraînent le développement de tendances technologiques et créent des opportunités d'investissements dans de nombreux domaines. Un tout nouveau marché est né, qui trouve aussi, peu à peu, l'acceptation des entreprises à la recherche de fonds. De nouvelles possibilités de sortie se développent; c'est aussi au cours de cette période qu'apparait le terme «F\&A » (« fusions et acquisitions »).

L'Etat découvre lui aussi le potentiel que représente pour lui le soutien à l'innovation ou l'aide à la création d'activité : de nombreux fonds de création sont constitués, comme le fonds de capital-investissement pour les entreprises technologiques (Beteiligungskapital für Technologieunternehmen, BTU), le European Recovery Program (ERP) ou les programmes INNO.

Phase 3 «Consolidation»: De nombreux business models de la nouvelle économie se sont avérés défaillants, ce qui entraîne finalement l'éclatement de la bulle Internet, avec des cours d'actions tombant en dessous de $5 €$ («Pennyactions »). Les investisseurs réagissent avec frilosité, de nombreuses sociétés de capital-risque quittant notamment le marché; les entreprises à la recherche de fonds se retrouvent confrontées à une pénurie de capitaux. La méfiance s'est également insinuée dans les entreprises. Les sociétés de F\&A, qui poussaient auparavant comme des champignons, se raréfient soudain. La confiance est clairement entamée.

Vient peu à peu le temps de l' "économie réelle », l'idée que seules les entreprises de technologie, véritablement viables, peuvent s'imposer sur le marché. L'Etat réagit lui aussi à la crise, mettant un terme aux dispositifs de soutien ou les adaptant au marché. On peut situer cette phase de consolidation entre mars 2000 et 2004.

Phase 4 «Croissance durable»: Le marché du financement se remet peu à peu de sa crise, ce qui se traduit en particulier par l'augmentation du nombre de sociétés de capital-investissement. Les sociétés nationales se montrent elles aussi plus matures. Les sociétés internationales renforcent leurs activités et c'est le début des levées de fonds. Bref : les investisseurs se remettent à miser sur le capital-investissement.

De leur côté, les entreprises reprennent peu à peu confiance. Avec les normes Bâle II, qui obligent les banques à avoir une base de fonds propres plus importante, les entreprises sont aussi confrontées de plus en plus souvent à des cotes de crédit et à des formes alternatives de financement, ce qui entraîne un profond changement dans le financement des PME. En outre, des secteurs tout à fait nouveaux conquièrent le marché, comme la biotechnologie et la technologie solaire.

Le soutien public fait un bond remarquable : le fonds de fonds ERP-EIF est mis en place; le fonds d'amorçage ERP et le fonds de création high-tech sont créés. Les bourses connaissent un nouvel essor, ce qui crée de nombreuses opportunités d'entrées en bourse, et même le marché des F\&A retrouve sa vigueur passée. La phase de croissance durable a commencé en 2004 et perdure jusqu'à maintenant.

Partant du classement opéré en Allemagne par BVK e. V., qui situe le capitalrisque (Venture Capital) dans la catégorie générale du capital-investissement (Private 
Equity), qui englobe également les financements LBO (Leverage buy out), l'évolution des placements en capital-investissement s'est déroulée ainsi depuis 1992 :

En 1993, un volume global de 517,3 millions $€$ provenant de fonds de capitalinvestissement était injecté dans des PME. En 2006, ce volume atteignait 3638 millions $€$, soit environ sept fois plus. Le niveau le plus élevé a été enregistré en 2000 et 2001 avec des volumes respectifs de 4451 et 4435 millions $€$. Alors que la part de capital-risque dans l'ensemble des opérations de capital-investissement atteignait son plus haut niveau en Allemagne dans les années 1999-2001, elle a connu un repli de l'ordre de $1 / 4$ à $1 / 3$ de l'ensemble des investissements après l'effondrement du nouveau marché. Or, c'est dans les premières phases de développement des PME que le recul du capital-risque est problématique, car il représente alors jusqu'à $10 \%$.

L'essentiel des capitaux a été injecté et continue aujourd'hui de l'être dans des transactions de LBO au volume important, qui ont une image négative dans l'opinion publique.

\section{Le marché de l'investissement en Europe en 2005-2006 et dans les années suivantes. Comparaison: France, Allemagne, Grande-Bretagne}

La levée de fonds par des sociétés de gestion spécialisée constitue la base des possibilités d'investissement de capital-risque. En 2006, cette méthode a permis de réunir 112,3 milliards $€$ en Europe pour des fonds de capital-investissement et de capital-risque, soit une augmentation de près de $50 \%$ par rapport à 2005 . Les Britanniques caracolaient en tête, avec près de 75,2 milliards $€$ à eux seuls ; la France atteignait 10,67 milliards $€$; et l'Allemagne représentait le maigre volume de 2,82 milliards $€$. La part des levées de fonds destinées à alimenter des fonds de capital-risque était très en retrait. En matière d'investissements, on trouve de nouveau la Grande-Bretagne en tête avec près de 41 milliards $€$, suivie de la France avec environ 10 milliards $€$ et l'Allemagne loin derrière, en troisième position, avec environ 3,5 milliards $€$.

L'on peut considérer 2006 comme une année record en matière de levée de fonds et d'activités d'investissements. Relativement à leur poids économique, l'Allemagne et la France n'y ont guère contribué. Si l'on observe la répartition des investissements, c'est surtout le secteur du capital-transmission (LBO) qui prédomine, ainsi que les financements de croissance avec des perspectives et des conditions assurées sur toute la planète. A l'échelle européenne, ce secteur représente environ $69 \%$; pour la France seule, ce sont même près de $80 \%$. Le capital-risque, qui concerne classiquement les premières phases (amorçage/ démarrage), est très en retrait. La valeur européenne moyenne se situe autour de $8 \%$, l'Angleterre se trouvant, avec environ $10 \%$, un peu au-dessus de la moyenne, et la France et l'Allemagne, avec respectivement $5 \%$ et $6,6 \%$, légèrement en dessous. La prise de risques financiers, qui représente aussi des chances pour les PME innovantes, tournées vers l'avenir, se dessine comparati- 
vement comme suit : pour la phase d'amorçage, les statistiques des associations interprofessionnelles nationales indiquent des chiffres situés entre 0 et 31 millions $€$ pour la France et l'Allemagne. La Grande-Bretagne se démarque, au moins dans les statistiques, avec 1,6 milliard $€$ d'investissements d'amorçage et 4,2 milliards $€$ d'investissements de démarrage.

La Grande-Bretagne est-elle la championne $d u$ monde $d u$ fonds d'amorçage? Les investisseurs allemands et Français sont-ils des lâches et des ratés? A chacune de ces questions, l'auteur souhaite clairement répondre par la négative. Premièrement, on peut supposer que les Britanniques ont éventuellement ajouté les investissements de démarrage au capital d'amorçage; et deuxièmement, on ne peut pas imputer les risques véritablement présents dans les phases initiales aux seuls investisseurs de capital-risque en France et en Allemagne : les conditions réglementaires sont encore trop défavorables à ces activités. D'éphémères aides fiscales et financières au financement des PME ne suffisent pas ; il est crucial de disposer du soutien de partenaires publics et privés à large échelle. Il en va en effet de notre avenir commun.

\section{Le capital-risque au niveau régional : le Brandebourg}

\section{$\mathrm{BC}$ Brandenburg Capital $\mathrm{GmbH}$ : la réussite grâce à un partenariat public-privé}

Pendant la première moitié des années 1990, dans tous les Länder, les PME se tournent avec un intérêt croissant vers le secteur du capital-risque, alors en plein boom. Au cours du processus de réunification de l'Allemagne, cette évolution a également concerné les nouveaux Länder, au premier rang desquels Berlin et le Brandebourg.

Le Brandebourg a pu bénéficier des premières expériences de son partenaire, la Rhénanie du Nord-Westphalie, mais également et en particulier de celles de Berlin, qui a lancé en 1989 un fonds d'amorçage soutenu par l'Union européenne (UE). Mais les précurseurs furent les sociétés d'investissements dédiées aux PME (mittelstandsorientierte Beteiligungsgesellschaften, $M B G$ ), qui créèrent en décembre 1992 la MBG Berlin/Brandenburg. Suivit Seed Capital Brandenburg GmbH (SCB) en novembre 1993, qui posa les bases des activités du capital-risque dédié aux phases de démarrage. Le fonds SCB permit à SCB GmbH d'investir 20 millions $€$ dans des PME, dans les phases de lancement. Il a reçu le soutien de l'Union européenne, de la KfW («Etablissement de crédit pour la reconstruction»), de la tbg («société d'investissement dans la technologie » à responsabilité limitée de la banque KfW des PME), des Länder de Brandebourg et de Saxe.

Dans les années 1990, lors de la mise en place de la SCB et de la société de capital-investissement pour le Brandebourg (KBB), ce Land est intervenu comme investisseur leader, motivant ainsi d'autres investisseurs.

Le fonds KBB, lancé en 1996, a été doté d'un volume de 40 millions $€$, avec une participation, là encore, du Land et de la KfW ou tbg. Mais le développement de 
l'activité d'investissement a connu une étape essentielle avec la création de BC Brandenburg Capital $\mathrm{GmbH}$, une banque qui opère depuis décembre 2000 comme établissement de gestion et holding, et gère aujourd'hui 5 fonds de capital-risque.

Il faut souligner que des investisseurs privés sont entrés à partir de 2001 dans le groupe $\mathrm{BC}$, porté par des sociétés publiques.

Le troisième fonds, BC Venture, a été créé en 2000 , avec un volume d'investissement de 45 millions $€$. Il a reçu le soutien du Fonds européen d'investissements (FEI), de la KfW/tbg, de l'ILB («banque d'investissement du Brandebourg ») et d'investisseurs privés.

En 2001, les Sparkassen du Land de Brandebourg ont pu lancer un fonds doté d'un volume d'investissement de 5 millions $€$. Ce fonds porte le nom de « Risikokapitalfonds der Unternehmensbeteiligungsgesellschaft der Sparkassen des Landes Brandenburg mbH », abrégé en S-UBG (Fonds de capital-risque de la société d'investissement des caisses d'épargne du Brandebourg).

Le cinquième fonds, BeteiligungsFonds Brandenburg $\mathrm{GmbH}$, a été créé en 2005. Son volume d'investissement est de 30 millions $€$, dont 20 millions sont cofinancés par des investisseurs privés. Les capitaux publics émanent à $75 \%$ du Fonds européen de développement régional (FEDER) et à $25 \%$ des aides du ministère de l'Economie du Brandebourg, injectés dans le fonds via l'ILB, au titre d'apport ciblé.

Après plus de 15 années d'activité, la $\mathrm{BC} \mathrm{GmbH}$ fait aujourd'hui figure d'exemple de partenariat public-privé (PPP) réussi.

\section{Les fonds FEDER, une source de financement pour des fonds de capital-risque}

Outre le développement de la $\mathrm{BC}$ et la continuité visible de l'offre de capital-risque pour les PME du Brandebourg, deux autres facteurs essentiels contribuent au succès de ce Land en matière de capital-investissement. D'abord, la constitution de fonds privés à haut degré de spécialisation et la mise à disposition d'autres offres de financement de la KfW via la $\mathrm{BC} \mathrm{GmbH}$, avec une orientation sur les PME innovantes. Puis, la mise à disposition, pour la première fois, d'aides FEDER pour des fonds de capital-risque par le Land de Brandebourg en 2005, comme base d'une offre de cofinancement d'investisseurs publics et privés renforçant considérablement le modèle de PPP. Le fonds FEDER suivant est prévu pour l'année 2009.

\section{Exemples de financements réussis par le groupe $B C$}

Depuis la constitution du SCB en 1993 et du KBB en 1996, la société BC GmbH a injecté plus de 100 millions $€$ dans 115 PME innovantes sous forme d'investissements directs, qui ont pu être complétés par près de 250 millions $€$ de cofinancement. Plus de 3000 nouveaux emplois qualifiés ont été créés dans ces entreprises; de nouveaux processus de développement technologiques et économiques y ont été mis en œuvre. Les investissements du fonds BC se sont concentrés sur les secteurs innovants et porteurs d'avenir au plan international : 
technologies de l'information et de la communication, sciences de la vie/biotechnologies, nanotechnologies, microélectronique/microsystèmes, mesures et asservissement, médias et nouveaux matériaux. Le volume d'investissement par PME a également évolué : ainsi, alors que le Seed Capital Fonds prévoyait un volume d'investissement moyen de 0,1 à 1 million $€$ au maximum, le $B F B$ a investi 0,3 à 1,5 million $€$ par PME. Les principales formes d'investissement sont les participations « ouvertes » et les participations « tacites ».

Ce sont surtout les participations aux quelques PME à forte croissance qui ont été décisives. Nous en présenterons cinq :

- Beyo : développement de systèmes de lecture mobiles pour les non-voyants ;

- Firstwood : traitement de bois des régions tempérés pour de meilleures propriétés, p. ex. résistance aux intempéries et aux nuisibles ;

- OD-OS : développement, production et commercialisation d'appareils de diagnostic et de traitement des maladies rétiniennes déclenchées par le diabète, en ophtalmologie ;

- PVflex : développement de technologies solaires intégrées ;

- Excentos : développement et commercialisation de conseillers produit en ligne, avec lesquels les boutiques en ligne et les fabricants de produits discutent sur Internet comme avec un super vendeur, optimisant leurs ventes.

Dans l'intervalle, sur les participations opérées depuis 1994, 20 PME ont été revendues à de nouveaux partenaires de la sphère entrepreneuriale et financière internationale, soit un produit supérieur à 30 millions $€$. On peut en déduire que l'investissement professionnel de capital-risque dans la nouvelle génération d'entreprises peut être une opération tout à fait profitable pour les sociétés de capital-risque.

\section{Le capital-risque pour les PME : la base de la nouvelle génération d'entreprises}

\section{Les PME et leur place dans l'économie nationale}

Des statistiques actuelles de la Commission européenne indiquent que les PME contribuent de manière significative à la satisfaction des objectifs définis en matière de politique économique et d'emploi.

Les micro-entreprises, qui sont selon la définition européenne de très petites entreprises employant moins de 10 salariés et ayant un chiffre d'affaires inférieur à 2 millions $€$, représentent plus de $90 \%$ des entreprises répertoriées en Europe et en Allemagne. Les PME représentent $5 \%$ supplémentaires, ce qui laisse moins de $5 \%$ pour les grandes entreprises et les groupes. En Allemagne, les PME créent $71 \%$ des emplois (elles y contribuent pour les deux tiers en Europe), et fournissent plus de $50 \%$ du produit national brut. A l'échelle euro- 
péenne, elles réalisent $60 \%$ du chiffre d'affaires des entreprises et représentent, selon les enquêtes statistiques de la Commission européenne, environ 165000 dépôts de brevets par an. Les PME fournissent donc un apport important en termes de croissance, de compétitivité, d'innovation et d'emploi. Les orientations prises en matière de soutien à l'économie, politique de l'emploi et, surtout, offres de financement en fonds propres ne sont, selon l'auteur, pas à la hauteur de ces réalités. Il reste à voir dans quelle mesure la crise financière actuelle et, à sa suite, la destruction de capital dans les grandes banques d'investissement et banques nationales en Allemagne et en France vont entraîner une diminution supplémentaire de l'offre de financement proposée aux PME.

\section{Les PME : critères de différenciation}

Comme cela a été montré plus haut, il faut considérer les PME de manière très différenciée. Il a fallu attendre janvier 2005 pour que la catégorie des microentreprises soit intégrée à la définition officielle des PME donnée par l'UE.

Selon l'UE, sont qualifiées de micro-entreprises les entreprises employant moins de 10 salariés et présentant un chiffre d'affaires ou un total de bilan inférieur à 2 millions $€$. Entrent dans la catégorie des petites entreprises les structures employant moins de 50 salariés et présentant un chiffre d'affaires annuel ou un total de bilan n'excédant pas 10 millions $€$. Les entreprises employant moins de 250 salariés et ayant un chiffre d'affaires inférieur à 50 millions $€$ ou un total de bilan inférieur à 43 millions $€$ relèvent de la catégorie « moyennes entreprises ».

D'une part, les mesures de soutien et les instruments de financement doivent correspondre aux dimensions des différentes entreprises, d'autre part, il faut souligner les exigences d'innovation et de croissance en particulier pour les micro- et les petites entreprises, qui constituent véritablement la nouvelle génération d'entreprises.

Des produits et des procédés nouveaux, avec les dépôts de brevets correspondants, naissent dans ces entreprises qui requièrent des fonds de capitalrisque en quantité parfois considérable et portent l'innovation pour le Mittelstand et la grande industrie. L'apport d'aides publiques européennes devrait par conséquent être proposé aux PME prioritairement sous la forme de capitalrisque ou de financement en fonds propres.

Pour ce faire, il est nécessaire et urgent d'étendre massivement les incitations à lever des fonds pour des fonds de capital-risque (amorçage, démarrage, première phase d'expansion)!

\section{Principaux acteurs impliqués dans le processus de financement de PME}

On peut fondamentalement répartir les principaux acteurs du processus de financement en trois groupes: les investisseurs de fonds assurent la mise à disposition du capital. Il faut toutefois au départ une structure durable et fiable 
d'acteurs publics et privés, d'une part pour soutenir le processus sans cesse renouvelé de mise sur pied de nouvelles entreprises compétitives au plan international et, d'autre part, pour faire confiance à l'estimation du risque et des chances de fournisseurs de capital-risque expérimentés. Le placement ciblé et préparé avec professionnalisme de capital-risque dans les PME est réalisé par des gestionnaires de fonds mandatés (capital-risqueurs) qui misent sur le rendement des capitaux engagés (entrepreneurs) : le choix des investissements se base sur une estimation précise des potentiels dont disposent les PME. Les PME mal gérées et peu innovantes ne sont généralement pas une cible d'investissement pour les fonds de capital-risque.

Enfin, il faut une convergence d'intérêts aussi large que possible entre les partenaires de financement, c'est-à-dire :

- les investisseurs,

- le gestionnaire de fonds et

- l'entrepreneur,

pour assurer la circulation des fonds. Les intérêts peuvent être de nature stratégique, économique, politique, entrepreneuriale. Il faut aussi prendre en compte les besoins financiers réels des PME au cours des différentes phases de financement, lesquels imposent une syndication des fonds publics et privés.

On distingue généralement différentes composantes dans le capital :

Capital d'amorçage: CR ; aides: A ; emprunts: $\mathrm{E}$; fonds propres des créateurs d'entreprises - FP ; part d'autofinancement : AF et capital de croissance : CC.

Pendant la phase d'amorçage, le capital d'amorçage constitue la plus grande partie du capital, suivi par les aides et les fonds d'emprunt. La part d'autofinancement est supérieure aux fonds propres des créateurs d'entreprises : $\mathrm{CR}>\mathrm{A}>\mathrm{E}>\mathrm{AF}>\mathrm{FP} .1$ à 3 millions $€$ sont généralement investis au cours de cette phase.

La phase de démarrage reste certes dominée par le capital d'amorçage, mais la part d'autofinancement dépasse déjà celle des capitaux empruntés et des aides : $\mathrm{CR}>\mathrm{AF}>\mathrm{E}>\mathrm{A}$. Les montants investis au cours de cette phase sont généralement de 3 à 7 millions $€$.

Pendant la première phase d'expansion, les investissements sont surtout financés sur le chiffre d'affaires : $\mathrm{AF}>\mathrm{CC} / \mathrm{CR}>\mathrm{E}$. Les montants d'investissement nécessaires sont supérieurs à 5 millions $€$.

Les montants indiqués ci-dessus sont issus des expériences faites par l'auteur de 1993 à 2008.

\section{Bilan}

- Le financement par capital-risque est la base sur laquelle va se construire la/les nouvelle(s) génération(s) d'entreprises, compétitives à l'échelle internationale ; 
- l'importance économique du secteur du capital-risque s'accroît du fait de l'actuelle crise internationale des marchés financiers et de l'affaiblissement des autres modes de financement qui en est résulté pour les PME ;

- malgré la polémique sur les « fonds sauterelles », qui concerne surtout le segment du capital-transmission (LBO), le secteur du capital-risque n'est pas perçu de manière négative par l'opinion publique (capitalrisque= garant de l'avenir)

- il reste essentiel de créer des conditions (juridiques, fiscales) plus favorables au développement du capital-risque en Allemagne, afin d'inciter constamment les investisseurs à financer de jeunes entreprises ;

- le capital-risque est aussi un « capital-chance». Les risques sont parfois supérieurs aux chances, mais comme on dit « no risk, no fun! ».

Traduction de Marie GRAVEY 



\section{Petites et moyennes entreprises, moteurs de croissance et d'innovation. Conditions et perspectives du rôle des PME dans le cluster optique de Thuringe}

\section{Christoph THIEME}

Christoph Thieme, chercheur à l'Institut de Conseil et de Recherche Sociale Appliquée (IPRAS) de l'Université d'léna, analyse ici les facteurs de succès des PME innovantes œuvrant sur le site optique et optoélectronique de Thuringe. Le principal défi de la branche, qui a plutôt bien réussi sa mutation structurelle depuis la chute du Mur, réside dorénavant dans la pénurie de main-d'œuvre qualifiée.

\section{Introduction}

Dans l'ensemble, les entreprises de l'industrie optique en Thuringe se sont bien développées dans les années qui ont suivi la chute du Mur et peuvent désormais, dans le Land mais aussi à l'extérieur, être considérées comme une branche exemplaire. Même si l'importance de la branche optique de Thuringe est plutôt faible aussi bien en termes d'emplois que par rapport à l'ensemble des entreprises du secteur manufacturier, la signification plus large des industries optiques en tant que technologie transversale essentielle ne doit en aucun cas être sous-estimée.

Après une difficile phase de survie et de consolidation suite au bouleversement politique et économique des années 1989/1990, les entreprises de la branche optique sont aujourd'hui devenues un important moteur de croissance et d'innovation en Thuringe. Avec 12000 salariés en 2007, ce secteur de l'industrie est certes encore loin de l'ancien niveau d'emploi, supérieur à 27000 salariés avant la chute du Mur dans la région d'Iéna et alentours, mais depuis plusieurs années, il se distingue par une santé économique robuste avec des taux de croissance des effectifs tournant autour de $5 \%$ en moyenne et une progression annuelle des chiffres d'affaires d'environ $10 \%$ (cf. Behr/Thieme, 2007).

Si le secteur manufacturier dans son ensemble a connu une évolution plutôt positive ces dix dernières années, celle de l'industrie optique en Thuringe ressort particulièrement. Dans la structure de la branche optique et optoélectronique organisée autour de petites entreprises, typique de l'économie estallemande, un modèle de production fortement innovant basé sur la qualification des ingénieurs et des travailleurs spécialisés semble s'être établi. Dans le présent article, on cherchera tout d'abord dans quelle mesure les petites et moyennes entreprises agissent comme importants porteurs et moteurs d'innova- 
tion autant que de croissance au sein de la branche optique et comment elles s'approprient ces deux éléments.

Dans la deuxième partie, on présentera les facteurs considérés comme déterminants pour le rôle des petites et moyennes entreprises dans la croissance et l'innovation tel qu'il a été présenté. L'attention se portera tout d'abord sur la base de main-d'œuvre qualifiée et sur la tradition, tandis que les points de l'infrastructure scientifique et technique ainsi que de la structure en cluster seront abordés en second.

En conclusion, la troisième partie s'articulera autour de l'esquisse d'un nouveau modèle est-allemand d'innovation et de production, pour s'interroger sur les conditions dans lesquelles le modèle présenté peut être porteur de développement futur. On envisagera en particulier l'évolution de la situation est-allemande en matière de personnel qualifié.

\section{La branche optique en Thuringe}

Même si le présent article traite essentiellement de l'évolution de l'industrie optique depuis la chute du Mur, élargir la perspective aux origines de l'industrie optique de Thuringe, qui remontent jusqu'au milieu du XIX ${ }^{\mathrm{e}}$ siècle, peut montrer quel rôle le lien entre l'économie et la science a joué dans son développement et comment on en est arrivé à la structure actuelle de la branche en petites unités.

C'est seulement dans ce contexte que l'on pourra correctement évaluer l'importance de la recherche et du développement dans les entreprises telle qu'on l'étudiera dans la seconde partie de cette section.

\section{Brève histoire de l'industrie optique}

Les origines de l'industrie optique en Allemagne sont idéalement illustrées par l'exemple de l'entreprise Carl Zeiss. Les deux ruptures de la Seconde Guerre mondiale et de la réunification en sont des éléments indissociables.

Ces origines remontent au milieu du $\mathrm{XIX}^{\mathrm{e}}$ siècle. Le fonctionnement des composants optiques était connu depuis des siècles, en particulier dans le cadre de la recherche astronomique, mais pour une percée industrielle, il manquait des procédures de fabrication généralisables. Pour cela, il fallait au préalable réformer la technique de l'optique pratique et obtenir les capacités techniques nécessaires, diffuser les bases théoriques et renouveler les principes pratiques relatifs au matériau. ${ }^{1}$ Seule la réalisation de ces conditions permit de franchir l'étape décisive suivante sur le chemin de l'industrialisation. Cette étape, menée par Abbe, consistait à fractionner le travail, ce qui augmenta la productivité et améliora la précision des instruments. La position de pionnier de l'entreprise

${ }^{1}$ Cf. Abbe, 1906, p.69, in : Büttner, 1993, p.17. 
Zeiss $^{2}$ résulte non seulement des capacités techniques et organisatrices d'Abbe, mais aussi des spécificités locales, en particulier de l'atmosphère intellectuelle propice aux relations entre l'université et le travail pratique de Zeiss (cf. Büttner, 1993). La symbiose entre économie et science est donc, depuis le début, un facteur constitutif de l'industrie optique. La coopération avec le chimiste Otto Schott, qui mettait à disposition des matières premières de grande qualité, permit de nouvelles améliorations dans ce domaine.

La croissance de l'entreprise se heurta très rapidement à des limites internes liées à l'organisation. Avec le changement de génération des années 1890, le pas fut franchi vers une direction professionnelle de la société. La transition entraîna également une diversification du portefeuille de produits jusqu'alors exclusivement consacré aux microscopes. ${ }^{3}$ La fondation Zeiss, mise sur pied au tournant du siècle, laisse deviner les prémices des instruments actuels de politique de gestion du personnel. Ainsi, Abbe considérait qu'assurer et transmettre le capital intellectuel était un objectif essentiel (cf. Abbe, 1906).

$\mathrm{Au}$ début du $\mathrm{XX}^{\mathrm{e}}$ siècle, la politique publique d'armement et l'augmentation correspondante de la demande envers l'industrie de l'armement s'accompagnèrent d'une internationalisation de l'entreprise Zeiss. La fin de la Première Guerre mondiale marqua un premier changement de cap dans le développement de l'industrie optique, le Traité de Versailles interdisant à l'Allemagne de produire du matériel de guerre et les entreprises devant se tourner vers de nouveaux domaines de production. Zeiss, par exemple, s'imposa dans la production de verres de lunettes et devint un fabricant essentiel de la branche. Une autre piste d'extension du chiffre d'affaires consista à conquérir et à développer les marchés étrangers. Cette tendance était également favorisée par la conjoncture mondiale ainsi que l'inflation de la monnaie allemande. Avec l'aggravation de la crise économique mondiale et la concurrence de plus en plus rude, une énorme pression économique poussait au renouvellement des produits et à la rationalisation. Ainsi, d'une part on mit sur le marché une série de produits innovants, tandis que d'autre part on réagit à partir de 1930 par des licenciements et des mesures de chômage partiel pour garantir la pérennité de l'entreprise au-delà de la crise mondiale.

La situation de l'économie allemande changea du tout au tout lorsque Hitler prit le pouvoir en $1933 .{ }^{4}$ La centralisation rassembla les entreprises d'optique et de mécanique de précision au sein d'un groupe public qui comptait environ 1300 entreprises pour un total de 100000 salariés. Pour chaque société, y compris Zeiss, cette mesure signifiait l'abandon d'une politique d'entreprise

\footnotetext{
${ }^{2} \mathrm{Au}$ moins sur le territoire de l'Empire allemand, et/ou en Europe.

${ }^{3}$ A la fin des années 1880, les longues-vues et les lunettes astronomiques, les instruments de mesure optiques ainsi que les lentilles photographiques s'ajoutèrent aux divisions de l'entreprise, cf. Büttner, 1993, p.20.

${ }^{4}$ La loi de cartellisation obligatoire du 15 juillet 1933 et la loi sur la préparation organique de l'économie allemande du 27 février 1934 mirent en place une politique économique centralisée qui régissait aussi bien les processus de production que l'organisation économique ; l'organisation de la production et la répartition du produit social étaient centralisées, cf. Eucken, 1952, p. 61 sv.
} 
indépendante. Comme lors de la Première Guerre mondiale, la politique publique d'armement se traduisit par une poussée de la demande qui cessa brutalement à la fin de la Seconde Guerre mondiale. En outre, l'industrie allemande de l'optique et de la mécanique de précision se trouvait dans une phase de maturité et de saturation sur les marchés essentiels des microscopes et des longues-vues, de sorte que l'après-1945 doit être considéré comme un nouveau départ.

Après la Seconde Guerre mondiale, l'évolution économique de l'industrie allemande de l'optique et de la mécanique de précision doit être considérée sous l'angle de l'Occupation par les forces alliées qui se manifesta à partir de 1950 par la partition de l'Allemagne en deux Etats. Cette évolution est particulièrement visible dans l'histoire de Zeiss: les forces américaines victorieuses avaient délocalisé la production à Oberkochen et l'expropriation des établissements de la Fondation Zeiss en 1948 scella la séparation de l'entreprise (cf. Büttner, 1993). Les principales caractéristiques de cette branche de l'industrie, qui se distinguait toujours par une forte tendance exportatrice, un haut degré de transformation et par conséquent un besoin en main-d'œuvre de haute qualité, restèrent cependant marquantes dans les deux parties de l'Allemagne, bien qu'à l'Est la productivité ait souffert de la perte de personnel qualifié. Entre 1950 et 1960, la production à l'Ouest augmenta proportionnellement plus que dans l'ensemble de l'industrie; dans la même période, le progrès technologique et les changements dans les habitudes de consommation entraînèrent une mutation structurelle non négligeable qui peut se résumer en deux éléments: recul de la production de biens de consommation et intensification des biens d'investissement. En 1961, le taux d'exportation tournait autour de $46 \%$. L'évolution a été tout aussi rapide sur le territoire de la RDA. Ainsi, en 1962 la production de Zeiss avait quadruplé, le taux d'exportation atteignant $44 \%$ cette année-là. En 1965, la direction du nouveau combinat industriel fut transmise à la société d'Etat VEB Carl Zeiss Jena, mettant pratiquement toute l'industrie optique et mécanique de précision sous une même autorité. Pendant la seconde moitié des années 1960, la croissance ralentit et les exportations continuèrent à progresser, mais surtout en direction des pays du COMECON.

La situation en RDA pendant les années 1960 et 1970 doit être replacée dans le contexte des mesures du NÖS ${ }^{5}$. A la fin des années 1960, on nota effectivement un bond dans la productivité du travail. Au début des années 1970, la commission de planification publique décida de développer les domaines de la mécanique de précision et de l'optique; de plus, il fallait construire des lignes de production pour les supports d'enregistrement électroniques

\footnotetext{
${ }^{5}$ Le Nouveau système économique de direction et de planification (Neues Ökonomisches System der Planung und Leitung der Volkswirtschaft, NÖSPL) débuta en 1963 et s'interrompit en 1972; son objectif était de faire sortir l'économie dirigée de la crise par des éléments économiques et en renforçant la responsabilité individuelle, cf. Kohli, 1994, p. 44.
} 
qui nécessitaient une forte intégration verticale en raison de l'absence de structure de fournisseurs. Si les produits soutenaient au moins partiellement la comparaison avec le marché mondial du point de vue technologique, il en résulta un retard impossible à combler en termes de productivité, établie d'après des calculs internes à environ un quart de celle des entreprises occidentales.

Avant la chute du Mur, le combinat Zeiss présentait une intégration verticale d'environ $80 \%$, due principalement à la pénurie de matériaux. Presque toutes les entreprises de mécanique de précision et d'optique sur le territoire de la RDA étaient subordonnées au combinat. Leur main-d'œuvre s'élevait à 58000 salariés en 1985 et augmenta jusqu'à environ 67000 personnes en 1989. En termes économiques, le combinat disposait d'une position de monopole dans l'espace du COMECON, de sorte que le commerce présentait une certaine sécurité en raison de la non-convertibilité des monnaies. Par ailleurs, le combinat s'était vu confier la mission de se procurer des devises, ce qui l'obligeait à s'exposer à la concurrence internationale.

Le budget important consacré à la recherche $-12 \%$ du chiffre d'affaires et $15 \%$ du personnel étaient employés au service $R \& D$ - n'empêcha toutefois pas le fait qu'une grande partie du portefeuille de produits ne satisfaisait plus aux normes internationales et était obsolète. Les sommes énormes englouties en vain dans le développement de puces-mémoires commercialisables retardaient le perfectionnement des gammes de produits classiques, de sorte que là aussi il était impossible de rattraper les normes internationales. La perte du monopole sur les marchés de l'Est ainsi que les conséquences de l'union monétaire en 1990 privèrent l'entreprise d'une base stable de débouchés et l'obligèrent à prendre, une fois de plus, un nouveau départ.

La branche se distingue aujourd'hui par une grande hétérogénéité. Avec les secteurs des produits d'optique oculaire, de la photonique et de la technique de précision ainsi que de la technique médicale, c'est une branche industrielle au caractère fortement transversal qui s'est développée. Le taux d'exportation reste typiquement élevé, atteignant presque $50 \%$ en $2007 .{ }^{6}$ Ces chiffres indiquent que l'industrie optique, médicale et mécatronique, avec une part de 4,6\% des entreprises et 3,6\% de la main-d'œuvre du secteur productif de Thuringe, est certes proportionnellement plutôt petite, mais dispose d'une force d'innovation supérieure à la moyenne et que son caractère transversal lui confère un rayonnement notable sur d'autres branches (cf. Schmidt/Behr/Thieme et al., 2003). C'est particulièrement valable pour la Thuringe, où cette branche est considérée

\footnotetext{
${ }^{6}$ Source : Thüringer Landesamt für Statistik (Office national de statistique du Land de Thuringe) : enquête de conjoncture dans le secteur minier et manufacturier pour la branche 33 - génie médical, mesure et asservissement, optique, horlogerie. Cette branche de l'économie est très similaire à l'industrie optique et optoélectronique qui a fait l'objet des enquêtes utilisées ici, mais pas totalement identique, ce qui explique la différence dans les taux d'exportation.
} 
comme une source d'espoir essentielle à laquelle sont associés d'autres potentiels de développement pour la région. ${ }^{7}$

Les PME, porteuses d'innovation et de croissance

Dans presque toutes les publications qui traitent du développement économique de l'Allemagne de l'Est depuis 1990, sa structure nettement plus fragmentée par rapport à l'économie ouest-allemande est considérée comme un inconvénient sérieux dans la compétition face aux concurrents occidentaux ou présentée comme un inconvénient local qui explique pourquoi le processus de rattrapage des entreprises est-allemandes en termes de productivité est en panne.

Les causes des inconvénients liés à la taille des entreprises sont multiples et résident dans des positions plus défavorables sur le marché et donc des marges inférieures. De plus, il existe aussi des inconvénients au niveau des achats, avec des remises sur volume moins importantes. D'autres explications sont la faiblesse des capitaux propres et le manque de notoriété au-delà de la région ainsi que des insuffisances dans l'établissement de certaines fonctions, par exemple au niveau du marketing et des ressources humaines dans les plus petites entreprises (cf. Behr/Schmidt, 2006).

Un inconvénient important qui ne manque jamais à cette énumération est le manque de ressources pour la recherche et le développement.

Par ailleurs, on s'accorde largement à considérer qu'en Allemagne de l'Est, à cause de la structure des entreprises, seules celles du Mittelstand peuvent être envisagées comme porteuses de croissance, car ce sont elles qui représentent le principal vivier d'emplois des entreprises. La capacité de ces entreprises à remplir leur rôle de porteur de croissance est souvent associée à leur capacité d'innovation (cf. Behr/Schmidt, 2005).

En 2007, la branche optique de Thuringe comptait au total plus de 160 entreprises et employait 12000 personnes. Son chiffre d'affaires se situait légèrement au-dessus de 2 milliards $€$, dont $59 \%$ réalisés à l'étranger. Pour $10 €$ de recettes, $1 €$ est réinvesti dans le secteur de la $\mathrm{R} \& \mathrm{D} .{ }^{8} \mathrm{C}$ 'est ainsi que, ces dernières années, la branche a pu étendre son volume de main-d'œuvre et son chiffre d'affaires. L'évolution depuis 2001 équivaut à une croissance annuelle de $5 \%$ en personnel et de $10 \%$ en chiffre d'affaires. La répartition des entreprises selon leur taille montre une prédominance des PME. Environ un tiers des entreprises entre dans la catégorie des très petites entreprises avec moins de 10 salariés. Environ $3 \%$ de tous les salariés de la branche travaillent dans ces entreprises. Représentant $37 \%$ du total, la plupart des entreprises

\footnotetext{
${ }^{7}$ Ainsi, le projet technologique du Land de Thuringe prévoit de renforcer non seulement l'industrie automobile, les microtechniques et le génie biologique ainsi que médical, mais aussi l'industrie optique ainsi que les infrastructures d'entreprises et de recherche.

${ }^{8}$ Les données indiquées ici et par la suite résultent d'un sondage réalisé dans l'industrie optique de Thuringe au printemps 2007 par l'institut IPRAS (FSU Iéna), qui interroge régulièrement les entreprises de la branche optique depuis 2001 ; la dernière enquête constitue la $4^{\mathrm{e}}$ depuis 2001.
} 
comptent de 10 à 49 salariés et regroupent tout de même $12 \%$ de l'ensemble de la main-d'œuvre. Un quart des entreprises se classe dans la catégorie allant de 50 à 249 salariés et emploie un tiers du personnel. Le groupe le plus important en termes d'emploi est celui des entreprises de 250 personnes et plus, où travaillent $58 \%$ des salariés dans $8 \%$ du total des entreprises.

\section{Graphique 1 : Evolution de la branche optique depuis 2000}

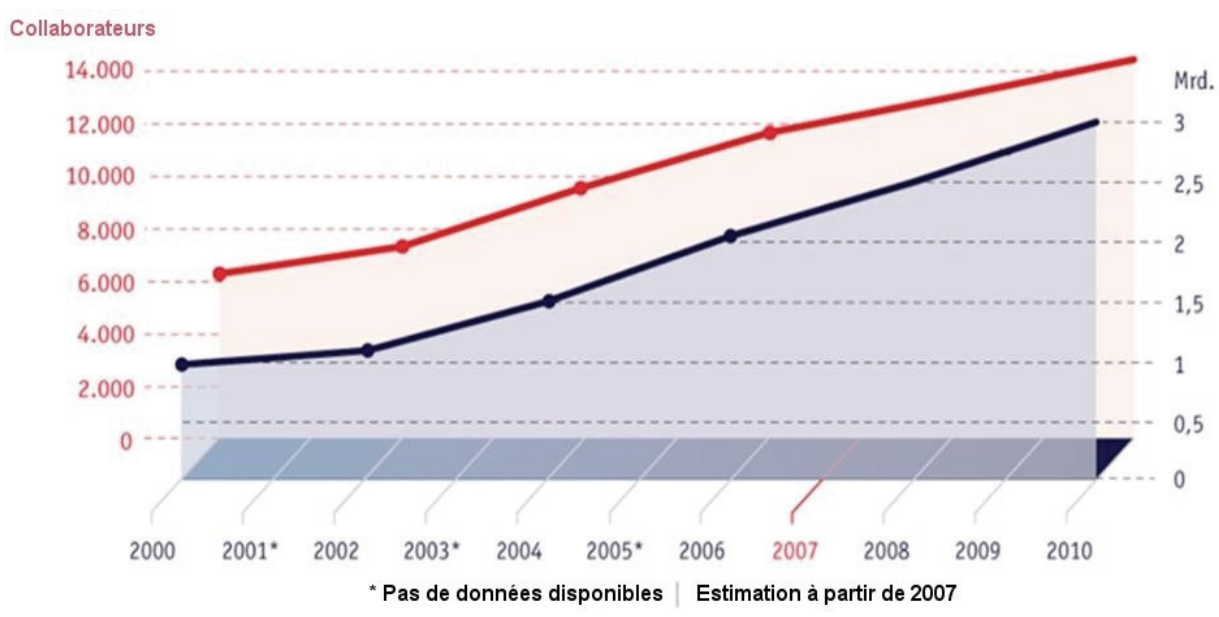

Source : Behr, Michael/Thieme, Christoph: Die optische Industrie in Thüringen - Hightechbranche auf Wachstumskurs, Iéna 2007.

Pour pouvoir identifier les porteurs de croissance au sein de la branche, il faut cependant adopter une approche différenciée. D'une part, il existe des différences notables dans l'évolution des entreprises par segment de taille ces dernières années ; d'autre part, la progression du chiffre d'affaires et du nombre de salariés ne repose pas seulement sur une croissance organique. Si l'on étudie l'évolution des entreprises par segment de taille, au premier abord aucun des segments ne se distingue par un dynamisme particulier. Aussi bien au niveau du nombre d'entreprises que du nombre de salariés, l'évolution des groupes répartis selon la taille de l'entreprise ne présente guère de différences entre 2003 et 2007, tous les segments se caractérisant cependant par une croissance plus ou moins forte comprise entre $32 \%$ et $76 \%$. En outre, le seul critère d'évolution de chaque segment dans une branche aussi dynamique ne peut donner qu'une image très déformée. Il existe plusieurs raisons à ce phénomène : tout d'abord, la croissance dynamique fait qu'il peut se produire d'importants décalages entre les différents segments pendant la période étudiée. Par ailleurs, malgré le recul des activités de création d'entreprise, il existe toujours de nouvelles créations qui se traduisent par une modification des segments inférieurs. De plus, ces dernières années de nombreuses entreprises ont déménagé sur de nouveaux sites. 
Un dernier facteur important pour l'évolution des segments, que l'on ne peut pas attribuer à la croissance organique des entreprises, est l'entrée de sociétés dans le domaine des technologies optiques suite à une diversification de leur offre de produits, ce qui entraîne leur prise en compte dans la branche. En raison de ces facteurs d'influence, l'évolution des différents segments ne peut pas être mise en parallèle avec la croissance des entreprises dans ces segments. Il est donc plus pertinent de considérer l'évolution des entreprises pour lesquelles on possède des données en termes de main-d'œuvre sur toute la période étudiée, de 2003 à 2007. On peut alors voir clairement dans quels segments de taille se trouvent les entreprises qui ont progressé par elles-mêmes. Tandis que les entreprises de moins de 10 salariés et de plus de 250 salariés ont eu tendance à stagner, voire à reculer légèrement, les segments médians de 10 à 249 salariés présentent la plus forte progression du personnel avec une croissance supérieure à $30 \%$. Ces petites et moyennes entreprises détiennent donc de toute évidence le rôle de porteurs de croissance (cf. graphique 2).

\section{Graphique 2 : Croissance organique des entreprises en fonction de leur taille (Données en valeurs absolues, croissance en \% selon la taille des entreprises en 2007)}

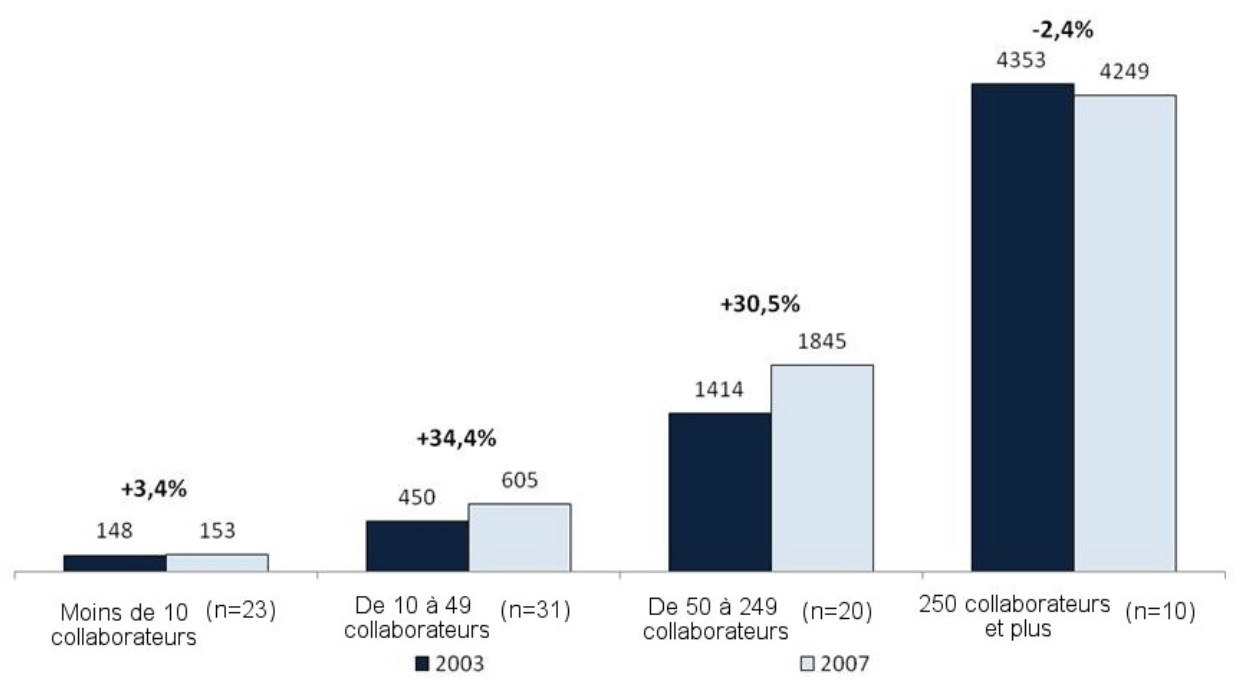

Source : propre enquête.

Comme on l'a montré précédemment, la R\&D ainsi que l'innovation jouent traditionnellement un rôle central dans la conception du modèle de production pour la plupart des entreprises de la branche optique, et ce, quelle que soit leur taille. Dans les petites et moyennes entreprises précisément, on en trouve un nombre plus élevé qui accordent une très grande importance à la recherche et au développement. Lorsque la taille des entreprises augmente, la proportion de ces 
entreprises recule légèrement, tout en restant remarquable à $40 \%$ dans le groupe des plus grandes entreprises (cf. graphique 3).

\section{Graphique 3 : Importance de l'innovation en fonction de la taille de l'entreprise Quel rôle pour la R\&D dans les entreprises ? (Données en \%)}

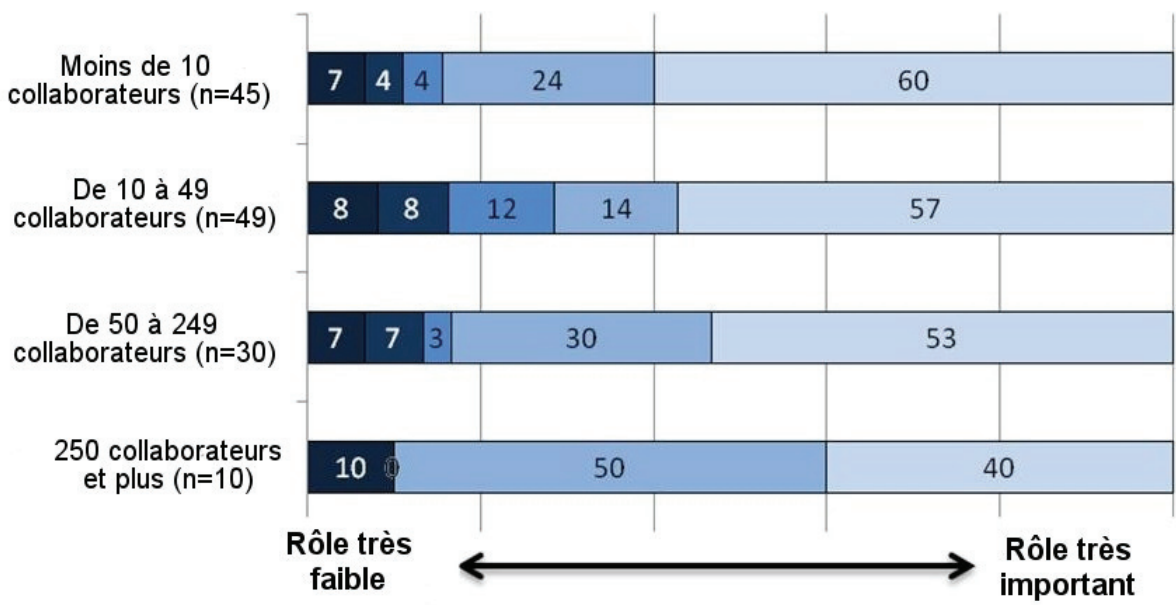

Source : propre enquête, 2007.

Le montant des investissements réalisés dans la recherche \& développement par rapport au chiffre d'affaires est un autre indicateur qui montre bien que ce secteur est primordial pour les petites entreprises. C'est dans les très petites entreprises que le pourcentage est le plus élevé avec $31 \%$. Les entreprises de 10 à 49 et de 50 à 249 salariés réinvestissent environ $15 \%$ de leurs recettes, tandis que la proportion s'établit à $9 \%$ dans les grandes entreprises de plus de 250 salariés. Même s'il est possible que des méthodes comptables différentes accentuent les écarts, ces valeurs montrent clairement quelle importance capitale les entreprises accordent au domaine de la R\&D.

Dans l'ensemble, on peut constater jusqu'ici que ce sont principalement les petites et moyennes entreprises qui assurent la croissance organique de la branche optique, se ménageant également une place très importante dans la recherche et le développement. Nous allons maintenant étudier les modalités et les conditions d'exercice de cette fonction au sein de la branche optique.

\section{Conditions nécessaires au rôle de porteur de croissance et d'innovation}

L'évolution impressionnante présentée plus haut est dans son ensemble le résultat d'une série de facteurs qui dépassent sans aucun doute les points présentés ci-après. On pensera principalement aux facteurs dits subjectifs tels que l'offre culturelle, la situation politique globale et la sécurité publique. Le choix des cri- 
tères présentés cherche cependant à résumer les points les plus importants. Il s'agit, d'une part, de facteurs directement liés au site d'Iéna et, d'autre part, de facteurs développés ou favorisés sciemment. On montrera ainsi que l'évolution économique peut être influencée, mais pas intégralement planifiée et donc qu'elle n'est pertinente qu'en harmonie avec les facteurs locaux existants.

\section{Tradition et potentiels humains}

Une condition essentielle à la force d'innovation et de croissance de toute la branche optique peut, comme on l'a déjà décrit, résider dans l'évolution historique de l'optique. Pour cette analyse, il est utile de faire la différence entre la tradition de l'industrie optique et la disponibilité d'un abondant capital humain bien formé après l'effondrement de la RDA. Ces deux facteurs permettent de répondre aux questions sur les raisons du bon développement de l'industrie optique après 1990. Au sujet du capital humain, on peut surtout citer, outre l'excellence disponibilité de main-d'œuvre spécialisée sur le marché, les conditions favorables en termes de politique de ressources humaines: salaires peu élevés, travailleurs peu exigeants mais très motivés (cf. Behr, 2000), tandis que le facteur tradition concerne surtout la conscience et le lien des acteurs avec l'optique. Par ailleurs, une série d'institutions a survécu à l'effondrement économique : on citera par exemple l'Université et la Fachhochschule Friedrich Schiller et leurs prédécesseurs. Il y a également eu quelques autres établissements de recherche dont l'histoire remonte bien avant la chute du Mur.

\section{Graphique 4 : Les facteurs de succès \\ (Données par catégorie en \%, $n=47$ )}

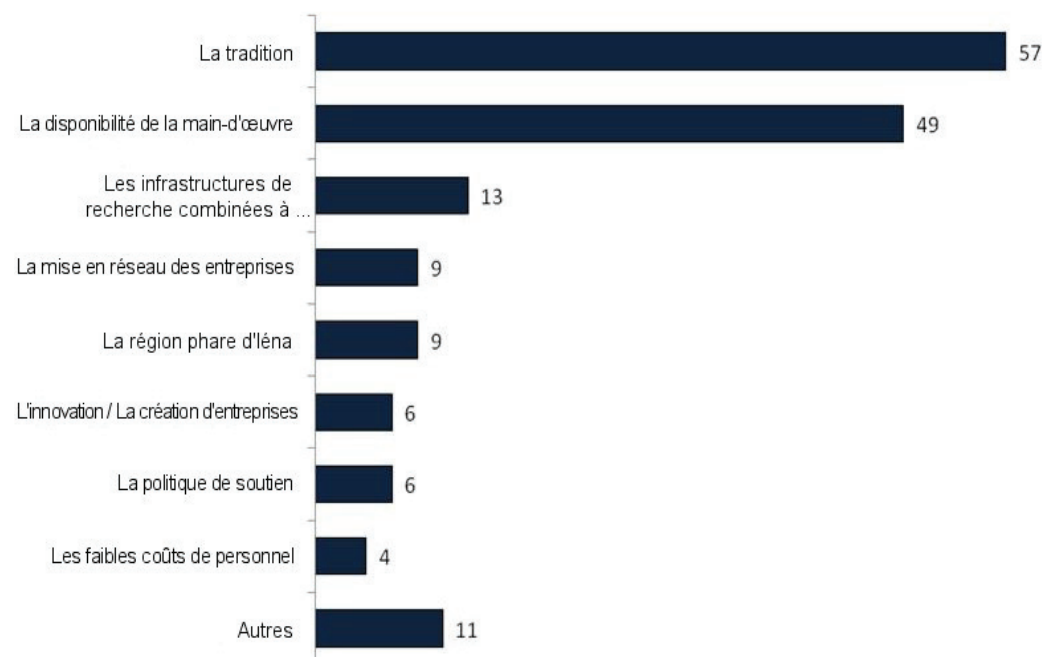

Source : propre enquête, 2004. 
Juste après 1990, le capital humain et la tradition ont été les facteurs décisifs pour la reconstruction de l'industrie optique, puisque les installations de production étaient largement obsolètes et les produits trop chers pour les principaux marchés historiques de l'ancien COMECON suite au changement de monnaie en 1991.

L'ancien combinat VEB Carl Zeiss Jena, avec ses 30000 salariés en 1989, a été scindé en deux entreprises, Carl Zeiss Jena GmbH et Jenoptik AG, qui employaient encore à elles deux environ 2000 salariés en 1991. Par la suite, une forte dynamique de création d'entreprises s'est installée, grâce à laquelle on compte aujourd'hui environ 160 entreprises en Thuringe. Une majorité de ces entreprises est directement dérivée de divisions de l'ancien combinat, et de nombreuses entreprises nouvelles ont également leurs racines chez Zeiss. La troisième source est la création de « spin-offs » d'établissements scientifiques. L'importance du volume de main-d'œuvre qualifiée et expérimentée se reflète aujourd'hui encore dans la structure d'emploi des entreprises. Au total, presque $90 \%$ des salariés sont ingénieurs ou travailleurs qualifiés, seul un salarié sur dix est sans qualification ou formé sur le tas. La comparaison en fonction de la taille des entreprises montre que les très petites et petites entreprises sont particulièrement bien pourvues en ingénieurs, leur proportion étant supérieure à $50 \%$, voire aux deux tiers (cf. graphique 5). C'est surtout en s'appuyant sur leurs excellentes ressources en main-d'œuvre qualifiée que les entreprises ont pu s'établir sur de nouveaux marchés.

\section{Graphique 5 : Qualification du personnel en fonction de la taille de l'entreprise (Données en \%)}

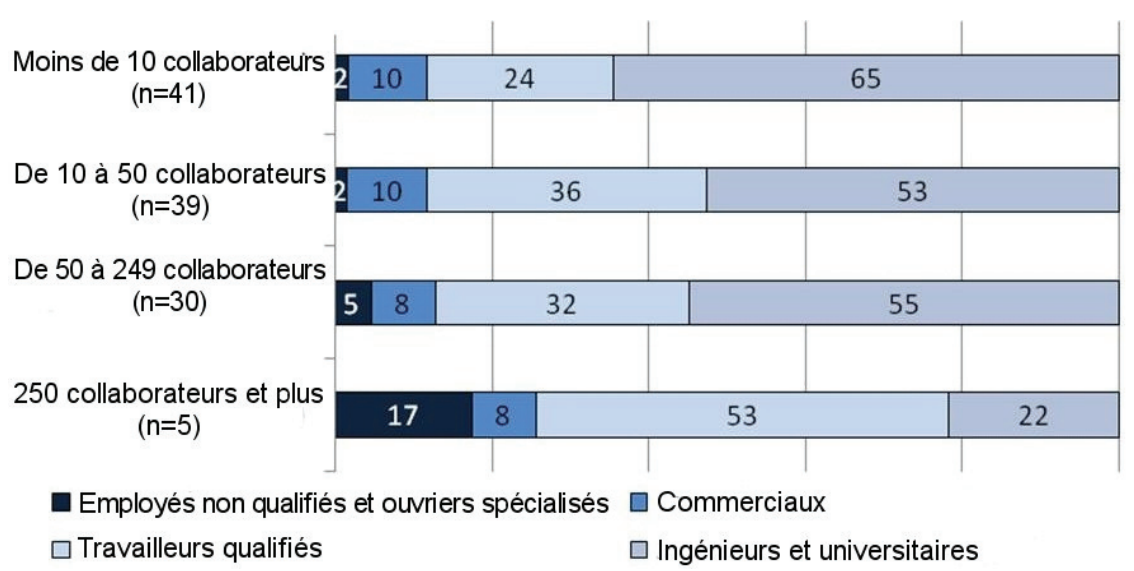

Source : propre enquête, 2007.

Bien que la main-d'œuvre qualifiée puisse être considérée comme la base du succès des entreprises, ce sont surtout les acteurs de leur création, provenant principalement de la deuxième génération de dirigeants de l'ancien combinat, 
qui ont joué un rôle décisif dans la conquête des nouveaux marchés. Ils ont eu une importance névralgique pour le développement des entreprises; ils étaient prêts à remplir leur mission de direction grâce à leur expérience non seulement technique, mais surtout administrative, car contrairement à la plupart des combinats est-allemands, ils disposaient déjà d'une longue expérience de la conduite de négociations, y compris avec les clients occidentaux, et aussi souvent du développement de projets de manière indépendante. Dans les premières années, ces acteurs ont assuré pratiquement seuls, outre la direction des entreprises, des missions capitales telles que l'acquisition de clientèle, la vente et la gestion du personnel. Et c'est surtout dans le domaine de la recherche et du développement que les chefs ont souvent joué le premier rôle, précisément dans les petites et moyennes entreprises. Tout compte fait, dans une entreprise sur deux de moins de 10 salariés, dans un tiers des entreprises de 10 à 49 salariés et dans un quart des entreprises de 50 à 249 salariés, le dirigeant joue toujours un rôle important d'initiateur des innovations. C'est dans les grandes entreprises de plus de 250 salariés que cette proportion est la plus faible, avec seulement $20 \%$ (cf. graphique 6).

\section{Graphique 6 : Initiateurs d'innovations (Données en \%)}

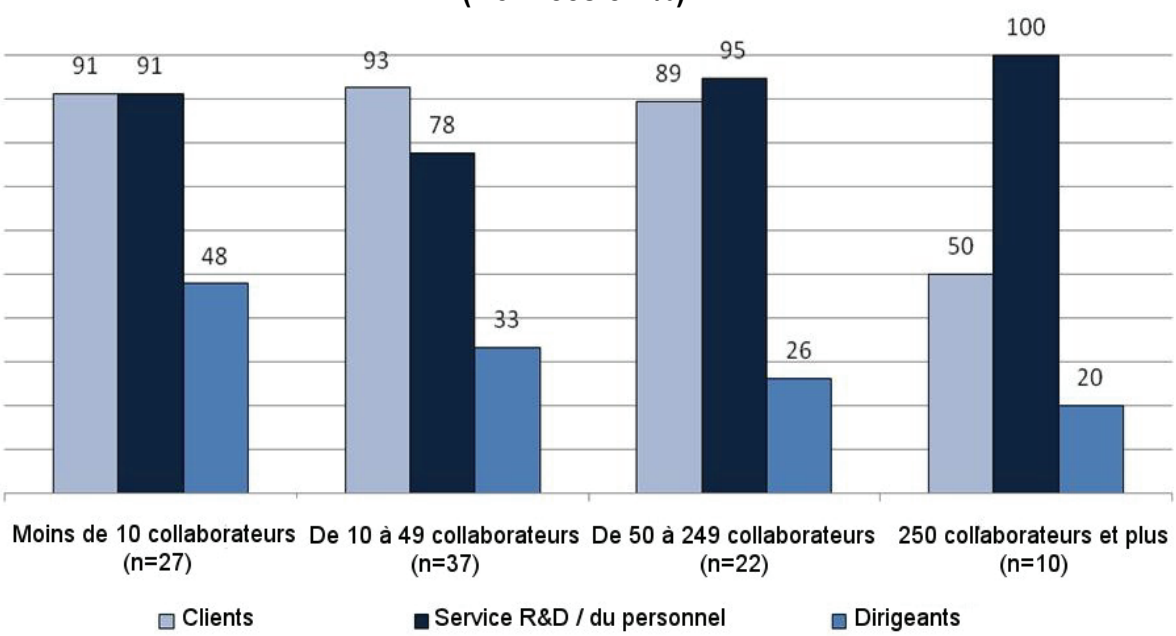

Source : propre enquête, 2007.

Cette situation des acteurs et de la main-d'œuvre a également représenté un facteur décisif pour l'évolution dynamique des entreprises de l'industrie optique dans les années qui ont suivi l'effondrement économique. Il a ainsi été possible de compenser, au moins en partie, les lourds déficits enregistrés en matière de rentabilité et d'accès au marché. Comme on le montrera ci-après, les acteurs de la création d'entreprises ont visiblement réussi à convertir l'in- 
tense pression de la concurrence sur les nouveaux marchés en développement de nouveaux produits.

\section{La pression de l'innovation}

Au début, la stratégie de nombreuses entreprises consistait principalement à se positionner sur le marché comme fournisseur de solutions personnalisées répondant aux problèmes des clients. Le travail de développement visait surtout à adapter des solutions connues aux exigences spécifiques des clients (cf. Behr/Schmidt, 2006).

Ce modèle de production s'est maintenu dans la plupart des entreprises. Dans les petites entreprises en particulier, les exigences spécifiques des clients sont le principal déclencheur du travail de développement (cf. graphique 6). Les entreprises les plus petites sont particulièrement soumises à la pression du marché. Environ une entreprise sur deux se déclare poussée par l'évolution du marché. D'un autre côté, il existe également un important groupe d'entreprises qui se sont ménagé des marges de manœuvre (cf. graphique 7).

\section{Graphique 7 : Pression du marché ou marges de manœuvre Vous sentez-vous poussé par l'évolution du marché ou existe-t-il assez de marges de manœuvre?} (Données en \%)

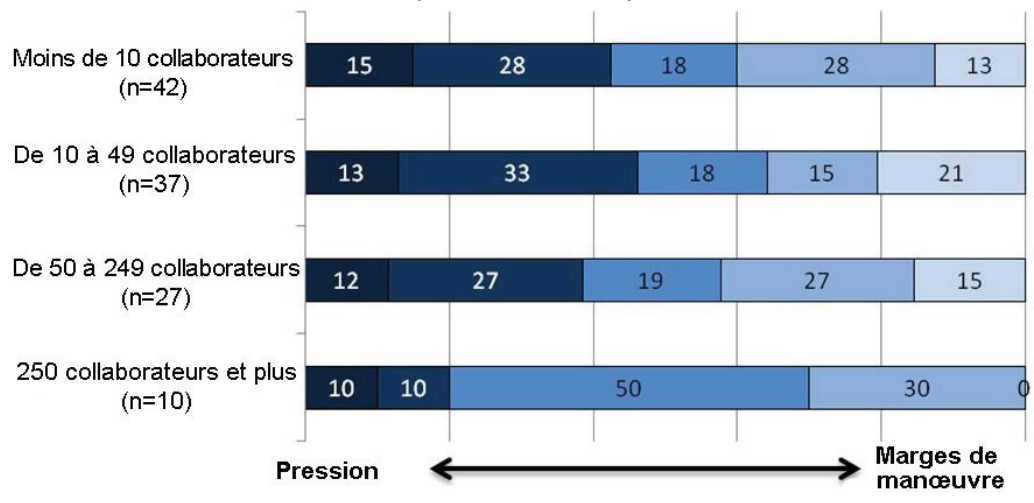

Source : propre enquête, 2007.

On constate en outre que le prix n'est apparemment un argument de vente décisif que pour un petit nombre d'entreprises. La grande majorité attribue son succès à la qualité technique de ses produits (cf. graphique 8).

Les travailleurs hautement qualifiés sont ainsi en mesure de développer des solutions exclusives pour lesquelles les clients sont prêts à payer des prix élevés. On ne cherchera pas ici à savoir dans quelle mesure ces rentes d'innovation sont réellement encaissées. Il est cependant très probable que la faiblesse des coûts salariaux puisse aussi expliquer le succès des entreprises. Toutefois, cet 
avantage devrait s'effacer dans les prochaines années, en raison de la pénurie croissante de main-d'œuvre de remplacement, en particulier en Allemagne de l'Est.

\section{Graphique 8 : Facteurs de succès commercial La réussite sur le marché dépend-elle du prix ou de la technologie? (Données en \%)}

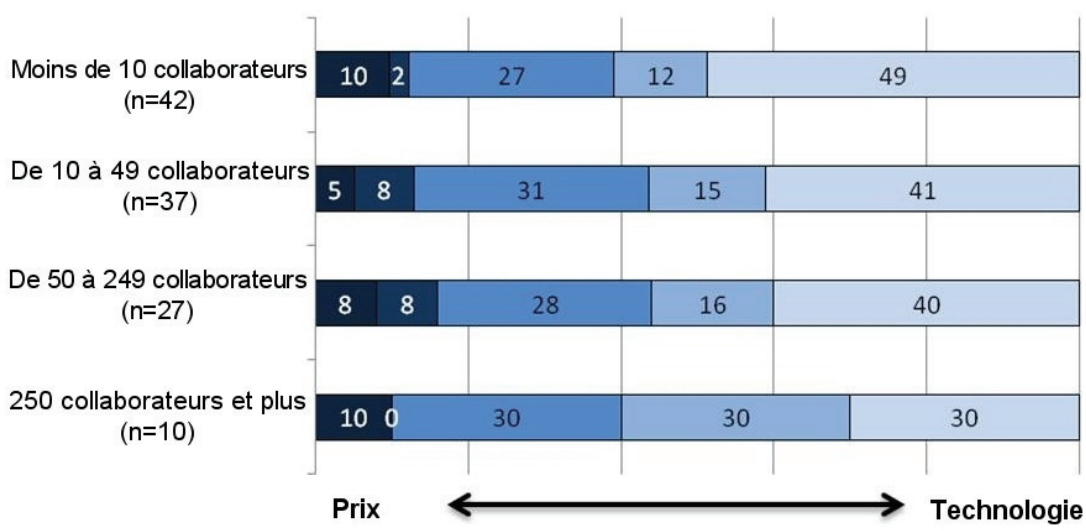

Source : propre enquête, 2007.

Cette grande proximité des clients, liée plus à la qualité des solutions techniques qu'à un prix avantageux, permet de conclure que le marché est stable. Cependant, dans de nombreuses entreprises la pression exercée par les clients pour l'innovation est principalement transmise par la personne du dirigeant, ce qui pourrait s'avérer un problème. Si cette fonction n'est plus exercée, en raison d'un changement de génération ou d'événements imprévus tels que la maladie ou un accident, cela pourrait avoir de graves conséquences pour l'entreprise.

\section{Infrastructures scientifiques et de recherche externes}

Une autre condition nécessaire pour que les petites et moyennes entreprises jouent le rôle de porteurs de croissance et d'innovation est la présence d'infrastructures externes de recherche et de formation. Ainsi, presque $90 \%$ des entreprises indiquent qu'elles ont recours à une aide extérieure dans le cadre de leur activité de R\&D. En examinant les chiffres des différents segments de taille, on remarque que les plus grandes entreprises reçoivent plus souvent un soutien continu. Environ $60 \%$ d'entre elles déclarent qu'elles doivent faire appel régulièrement à une aide externe, tandis que pour les autres c'est au minimum une aide occasionnelle. Mais dans les petites entreprises aussi, la proportion est très élevée. Dans respectivement $20 \%$ et $10 \%$ des entreprises des premiers segments de taille, l'éventail des tâches est assez réduit pour qu'une coopération avec des institutions externes soit considérée comme superflue. Cependant, presque toutes les entre- 
prises interrogées déclarent travailler avec des établissements externes. Il existe cependant des différences notables : alors que pour plus des deux tiers des PME, la coopération avec d'autres entreprises joue un rôle important, cette forme de collaboration est nettement moins marquée dans les grandes entreprises. Cela pourrait s'expliquer par leur position à l'extrémité supérieure de la chaîne de création de valeur, où une collaboration en matière de R\&D avec les soustraitants semble rarement utile. Par ailleurs, les entreprises situées à un niveau similaire de la chaîne de valeur ont tendance à entrer en compétition, ce qui rendrait difficile une coopération en matière de R\&D.

Les principaux partenaires de recherche et développement sont donc majoritairement des instituts universitaires ainsi que des établissements de recherche extra-universitaires. L'intensité de la coopération augmente quelque peu avec la taille des entreprises, cependant cette hausse ne peut être attribuée qu'en partie à une plus forte tendance vers la coopération en matière de recherche. En effet, les grandes entreprises ont plus de possibilités de mettre en œuvre des projets de coopération en raison de leur structure plus différenciée (cf. graphique 9). La proportion comparativement élevée de petites entreprises montre plutôt qu'une forte participation des petites et moyennes entreprises est tout à fait de l'ordre du possible. Les enquêtes réalisées dans d'autres branches d'activité en Allemagne de l'Est ont toujours montré des carences dans l'intensité de coopération de ces groupes d'entreprises (cf. le projet EFA III). D'autres interlocuteurs en cas de problèmes et de questions liés à la R\&D sont les réseaux, qui assurent une coordination très importante justement pour les entreprises ne disposant pas de contacts avec des établissements scientifiques (cf. Behr/Schmidt, 2007).

\section{Graphique 9 : Partenaires des entreprises pour la recherche A qui vous adressez-vous en matière de R\&D ? (Données en\%)}

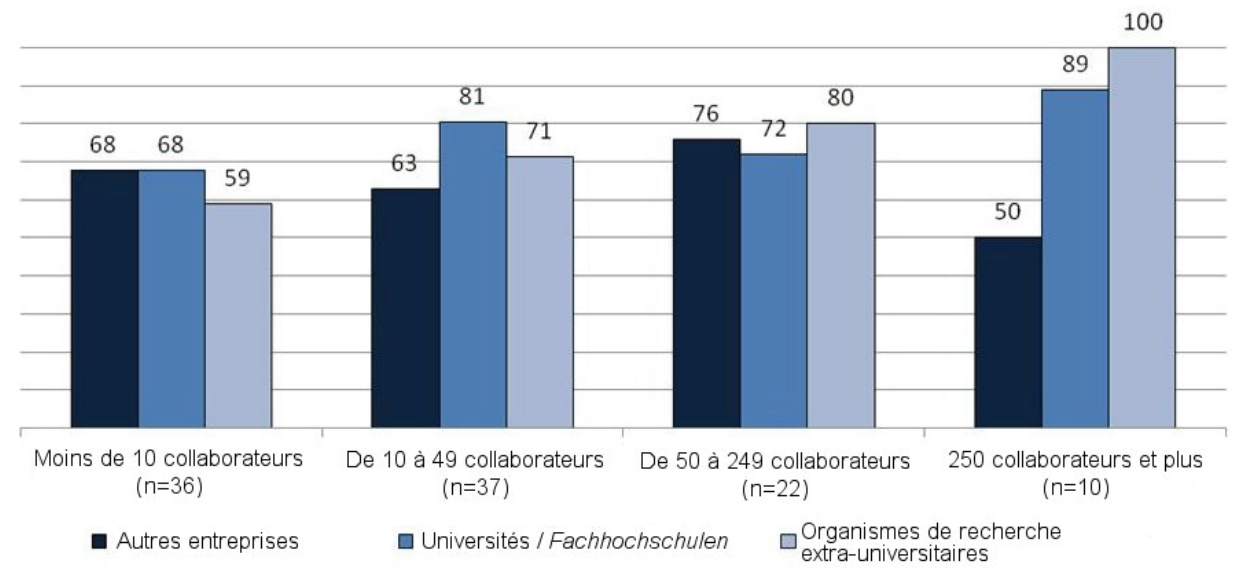

Source : propre enquête, 2007. 
L'étude des formes de coopération montre également que c'est d'abord dans le segment des petites et moyennes entreprises qu'une coopération durable est pratiquée. La proportion est la plus élevée dans les très petites entreprises de moins de 10 salariés, presque une entreprise sur quatre indiquant pratiquer une coopération durable en R\&D. Ce chiffre peut être considéré comme un indice du rôle important que jouent dans ce domaine les contacts personnels entre les dirigeants d'entreprise et les chercheurs. Dans les plus grandes entreprises, il existe plus de coopérations ponctuelles par projet et donc des partenaires plus changeants (cf. graphique 10).

Les entreprises ont accès non seulement à Iéna, mais aussi à Ilmenau, le deuxième pôle de l'industrie optique en Thuringe, à un réseau dense et bien développé d'établissements de formation et de recherche dont les racines remontent jusqu'aux origines de cette industrie et qui a été largement renforcé depuis 1990. En 2007, 600 scientifiques travaillaient sur des domaines relevant des technologies optiques. On citera principalement trois établissements d'enseignement supérieur: l'Université Friedrich Schiller, la Fachhochschule d'Iéna ainsi que l'Université technique d'Ilmenau. En outre, le Campus Beutenberg d'Iéna accueille l'Institut für Optik und Feinmechanik (IOF, Institut Fraunhofer d'optique et de mécanique de précision) et l'Institut für Photonische Technologien (IPHT, Institut de technologies photoniques), deux établissements qui étudient principalement les questions technologiques liées au domaine optique. Il existe également à Ilmenau un Institut für Digitale Medientechnologie (IDMT, Institut de technologie des supports numériques) et un Anwendungszentrum für Systemtechnik (AST, centre d'application des systèmes), deux instituts Fraunhofer à dominante optoélectronique. S'y ajoutent d'autres instituts moins importants ainsi que des centres de recherche collaborative et des écoles doctorales.

\section{Graphique 10 : Formes de coopération (Données en \%)}

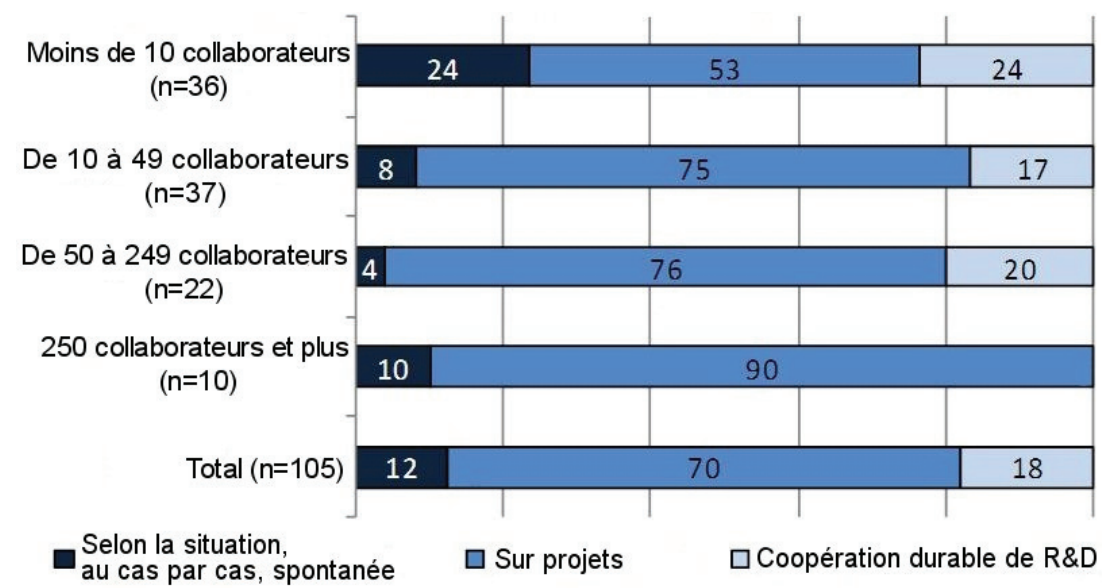

Source : propre enquête, 2007. 
C'est surtout en raison de l'étroitesse et de la durée des relations personnelles existantes entre les acteurs des établissements scientifiques et des entreprises que s'est établie dans la région une interaction efficace entre ces acteurs. Il ne faut pas sousestimer l'importance des acteurs des réseaux pour la (ré)activation des relations de coopération personnelles. Le réseau OptoNet, en particulier, a joué un rôle décisif pour renforcer la sensibilisation aux possibilités de la région et pour rassembler des partenaires régionaux. Cependant, des problèmes pourraient survenir au moment du retrait des porteurs du réseau, lorsqu'il faudra organiser leur succession. Les initiatives de réseau auront là une mission capitale à remplir pour transmettre les réseaux à la génération suivante.

\section{Une structure en cluster}

Comme il ressort des points précédents, il existe au sein de l'industrie optique et optoélectronique de Thuringe un tissu serré reposant sur les relations personnelles entre entreprises, établissements scientifiques et instituts universitaires. Par ailleurs, l'étroitesse des relations entre les entreprises elles-mêmes représente une particularité. Si l'on compare l'intensité des relations régionales en Thuringe à celle des autres sites optiques, c'est particulièrement évident: dans toutes les dimensions, la part des entreprises disposant d'un fort ancrage régional est nettement supérieure à celle des autres sites optiques d'Allemagne. C'est d'autant plus net dans les catégories des fournisseurs et des partenaires de coopération : tandis qu'en Thuringe, tout juste un tiers des entreprises possède son principal contact client dans la région, ce taux est seulement de $12 \%$ en moyenne sur l'ensemble des sites optiques en Allemagne. Le principal partenaire de coopération est également situé en Thuringe pour une entreprise optique sur deux de la région, alors que sur les autres sites, c'est seulement une entreprise sur quatre qui a un partenaire dans la région (cf. graphique 11).

Outre la proximité régionale des relations d'affaires, il existe aussi, comme on l'a déjà mentionné plus haut, une coopération très étroite en matière de défis techniques. Pour les petites et moyennes entreprises surtout, l'adaptation aux besoins du client représente la forme de production typique. Plus de $70 \%$ des entreprises de moins de 250 salariés déclarent produire exclusivement ou majoritairement à partir d'indications spécifiques des clients. Simultanément, il n'existe en Thuringe aucune structure de fournisseurs exclusivement consacrée à un producteur final. Cela tient d'une part au taux d'exportation élevé ; d'autre part, il n'existe aucun véritable producteur final à l'extrémité de la chaîne de valeur, sur lequel les autres entreprises régionales s'alignent. Au contraire, la branche se caractérise par une grande diversité de produits au niveau des composants complexes, des systèmes préintégrés et de l'intégration de systèmes, ce qui évite des situations de concurrence directe à un même niveau de la création de valeur. Au final, cela facilite la naissance de projets de coopération entre les entreprises (cf. Schricke, 2007). 


\section{Graphique 11 : Proximité régionale des relations d'entreprise Poids des partenaires régionaux de coopération et d'affaires (Données en \%)}

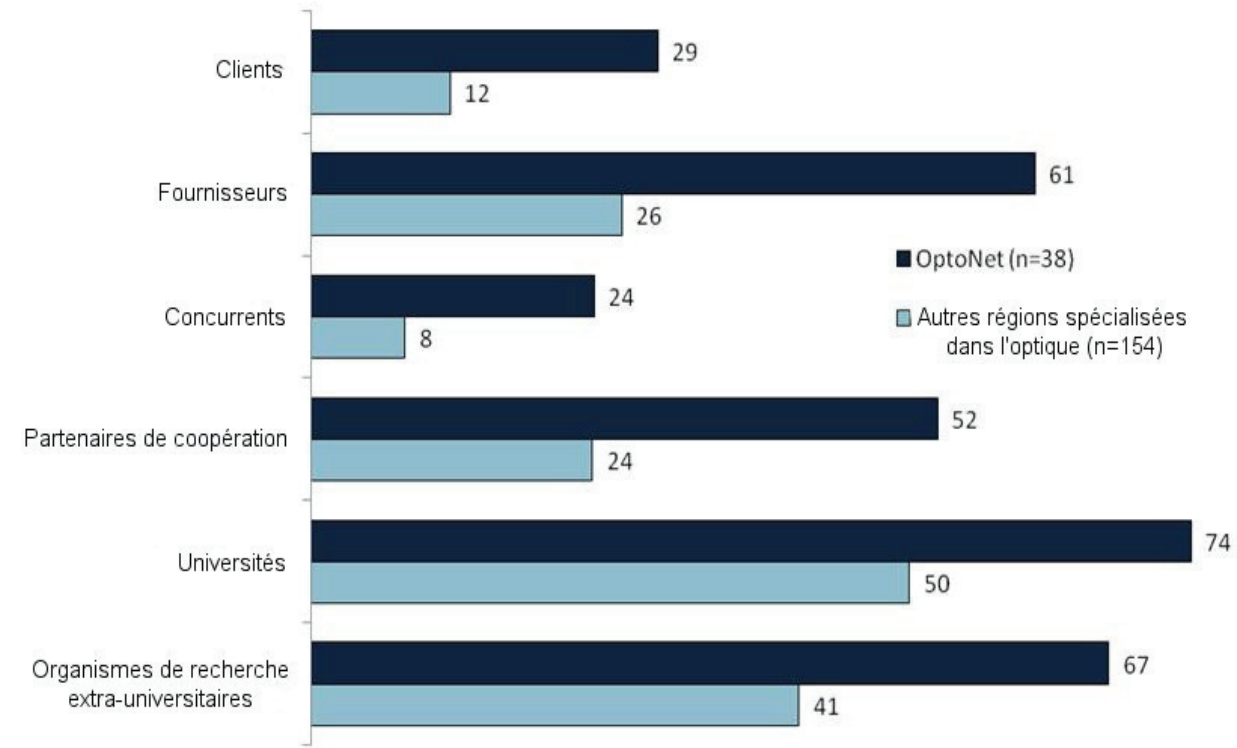

Source : propre enquête, 2005.

DANS L'ENSEMBLE, L'ÉVOLUTION de la branche optique et optoélectronique présentée ici incite à l'optimisme. On y voit des entreprises misant fortement sur l'adaptation aux besoins des clients, mais qui parviennent à se ménager des marges de manœuvre pour développer leurs propres produits et se donner ainsi une chance de sortir d'un rapport très étroit avec le client et donc de se libérer d'une forte dépendance envers un seul ou quelques clients. Pourtant, même si les entreprises ont gagné un meilleur accès aux marchés et donc une position plus favorable dans les négociations, ce sont précisément ces relations intensives avec les clients qui constituent la base du développement ultérieur de ces entreprises, car c'est toujours là que les principaux résultats de l'innovation sont obtenus. Comme les entreprises misant largement sur des composants et des produits finis standardisés ne jouent qu'un rôle mineur, il est donc peu probable que cette branche souffre exagérément des variations conjoncturelles. En outre, l'environnement institutionnel ainsi que les structures de cluster semblent soutenir le développement. En ce qui concerne l'industrie optique de Thuringe, le processus de transformation entamé après la chute du Mur a bien fonctionné.

Néanmoins, la branche s'expose à des problèmes non négligeables et à des tendances qui pourraient menacer sa progression. L'évolution démographique et 
surtout la problématique migratoire particulière à l'Allemagne de l'Est pourraient poser aux entreprises un problème de main-d'œuvre qualifiée dans les années à venir. Pour certains groupes de main-d'œuvre, il existe déjà aujourd'hui de véritables goulots d'étranglement qui pourraient se transformer en une véritable pénurie structurelle ces prochaines années, d'abord au niveau des jeunes sortant de l'enseignement secondaire, puis à celui des étudiants. Même si la croissance des dernières années a permis à de nombreuses entreprises d'établir des procédures de recrutement et d'intégration de nouveaux salariés, à l'avenir certains postes ne seront plus pourvus ou bien le seront à des conditions nettement différentes. En plus des postes non pourvus, l'écart croissant des rémunérations entre les salariés de différentes générations d'une part et entre les groupes de qualification d'autre part pourrait détériorer le climat des entreprises. Cela pourrait empêcher ou au minimum entraver nettement les missions de transmission d'expérience et de connaissances cruciales au moment du changement de génération. Les marchés de main-d'œuvre qualifiée étant encore très bien pourvus ces dernières années, de nombreux acteurs pourraient ne pas encore avoir pris pleinement conscience de la gravité de la situation.

Traduction de Marie-Céline GEORG

\section{Indications bibliographiques}

ABBE E., «Gedächtnisrede zur Feier des 50jährigen Bestehens der optischen Werkstätte», discours tenu le 12 janvier 1896, in ABBE E. , Sozialpolitische Schriften, G. Fischer, Iéna, 1906 p. [n.c.]

BÜTTNER S., Die Wettbewerbssituation in der feinmechanischen und optischen Industrie. Eine markttheoretische Analyse unter besonderer Berücksichtigung des Unternehmens Carl Zeiss, P.C.O.-Verlag, Bayreuth, 1993

BEHR M., THIEME C., Die optische Industrie in Thüringen - Hightech-Branche weiter auf Wachstumskurs, OptoNet e.V., IPRAS e.V, Iéna, 2007

BEHR M., SCHMIDT R. (ed), Potenziale und Beiträge ostdeutscher Hochschulen und Forschungseinrichtungen bei der Entwicklung regionaler Innovationspotenziale am Beispiel von Clusterbildungsprozessen im mitteldeutschen Raum, rapport de clôture du projet homonyme, aussi intitulé EFA III, pour le compte du ministère fédéral des Transports, de la Construction et des Affaires urbaines (BMVBS), à paraître dans les Jenaer Beiträge zur Soziologie, cahier 20 (conjointement avec SCHMIDT R., EHRLICH M., THIEME C., 2007

BEHR M., SCHMIDT R. (ed), Aufbau Ost - Innovation durch Kooperation und Fachkräfteentwicklung, Institut für Soziologie der Friedrich-Schiller-Universität Jena, Iéna, 2006 (Jenaer Beiträge zur Soziologie, cahier 18)

BEHR M., SCHMIDT R., Aufbau Ost - Betriebliche und überbetriebliche Erfolgsfaktoren im verarbeitenden Gewerbe Ostdeutschlands, Institut für Soziologie der Friedrich-Schiller-Universität Jena, Iéna, 2005 (Jenaer Beiträge zur Soziologie, cahier 16)

Behr M. , Engel T., Thieme C., Die Optische Industrie in Thüringen - starker Mittelstand setzt auf Export. Eine Studie des Kompetenznetzes für optische Technologien, Institut für Soziologie der Friedrich-SchillerUniversität Jena, Iéna, 2005

BEHR M., «Ostdeutsche Arbeitsspartaner», Die politische Meinung. Monatszeitschrift zu Fragen der Zeit, $\mathrm{n}^{\circ}$ 369 , août 2000 , p. $27-38$ 
BeHR M., ENGEL T., «Entwicklungsverläufe und Entwicklungsszenarien ostdeutscher Personalpolitik», in PaWlowsKy P., WILKENS U., Zehn Jahre Personalarbeit in den neuen Bundesländern. Transformation und Demographie, Hampp, Munich/Mering, 2001, p. 255-278

BRAUNSPERgER M., Feinmechanische optische sowie Uhrenindustrie. Strukturelle Probleme und Wachstumschancen, Duncker \& Humblot, Berlin/Munich, 1964

EUCKEN W., Grundsätze der Wirtschaftspolitik, Mohr/Siebeck,Tübingen, 1952

KOHLI M., «Die DDR als Arbeitsgesellschaft? Arbeit, Lebenslauf und soziale Differenzierung», in KAEBLE H., KOCKA J., ZWAHR H. (ed), Sozialgeschichte der DDR, Klett-Cotta, Stuttgart, 1994, p. 31-61

SCHMIDT R., BeHR M., ThIEME C. et al., Die optische Industrie in Thüringen - Zukunftsperspektive und Beschäftigungsentwicklung, Institut für Soziologie der Friedrich-Schiller-Universität Jena, Iéna, 2003

SCHRICKE E., Lokalisierungsmuster und Entwicklungsdynamik von Clustern in der optischen Technologie in Deutschland, wvb, Berlin, 2007. 


\title{
L'intégration de la problématique du Wissensmanagement
}

\author{
Isabelle BOURGEOIS
}

Une problématique dont il est rarement tenu compte dans l'analyse de la compétitivité des PME et de leur place dans le système d'innovation allemand est celle du "Wissensmanagement ॥. Elle est pourtant cruciale, car elle est l'un des éléments moteurs de l'innovation. Si le concept est relativement répandu en Allemagne, il est en revanche presque inconnu en France. Cette divergence révèle les différences foncières qui s'observent dans les cultures et approches de l'innovation de part et d'autre du Rhin. C'est donc de propos délibéré que j'ai choisi de ne pas traduire le terme "Wissensmanagement ». L'usage du terme allemand permettra ainsi de mieux garder présent à l'esprit que si les systèmes d'innovation nationaux reposent sur des structures et des flux entre celles-ci, ils sont avant tout déterminés aussi par une culture - ou plutôt : un système culturel.

\section{Management du savoir en France et en Allemagne : deux systèmes et deux visions du monde}

J'aurais certes pu opter pour l'appellation française la moins éloignée, à savoir "knowledge management ». Mais si ce terme semble à première vue la traduction littérale de "Wissensmanagement », il ne recouvre pas la même réalité. De surcroît, il ne se laisse guère traduire en français lui non plus. Ce terme très prisé outre-Rhin, et par-delà, dans tous les pays industrialisés, désigne généralement tout un ensemble d'outils et de systèmes informatiques destinés à collecter, hiérarchiser, faire circuler l'information au sein d'une entreprise pour accroître la performance globale de cette dernière. Ces outils et méthodes, connus aussi sous des appellations comme «business intelligence » ou " corporate performance management 》, servent à améliorer le pilotage de grandes entreprises ou groupes œuvrant dans un contexte de globalisation des activités et dont la structure constitue le plus souvent un ensemble très éclaté d'entités plus petites, voire de fonctions, qu'il convient de relier par le biais d'une communication plus efficiente de l'information. Autrement dit, l'objectif de performance globale appelle une nouvelle gestion du flux d'informations afin de faire émerger ou de développer une nouvelle culture de la transparence au sein d'une structure organisationnelle hautement complexe; cette transparence étant seule à même d'assurer la cohésion de tels ensembles disparates.

Le seul lien détectable avec l'innovation repose dans ce cas sur la mise en œuvre des TIC. Les méthodes et outils du knowledge management ont connu un large engouement en Allemagne à partir des années 1990, époque aussi de la promotion du «venture capital » et de nouvelles politiques d'innovation. Cette époque, où était née la «bulle Internet» a vu se multiplier les développeurs de logiciels et les structures de conseil liés à une approche hautement informatisée 
du «management du savoir». Mais les entreprises qui ont eu recours à ces services nouveaux, donc innovants, ont assez vite été déçues par les coûts, disproportionnés en regard des promesses de performance, des systèmes informatiques afférents. L'enjeu ne valant pas l'investissement, a fortiori dans une PME où les voies de circulation de l'information ne sont jamais longues, ces outils sont depuis boudés - sauf, bien sûr, dans le seul secteur où ils ont une justification 'd'outil de production' moderne : la logistique, dont le business modèle repose par définition sur la gestion de données.

Les Allemands - pouvoirs publics comme entreprises - se sont donc assez vite détournés d'un concept qui tenait plus d'un phénomène de mode à l'ère de la «bulle Internet» et qui, surtout, reposait sur une confusion. Ce Wissensmanagement-là permet certes d'améliorer le management des informations - autrement dit, le management de données. Mais les informations ou données ne sont pas le savoir. Et sur un site dédié (www.wissenmanagen.net), le ministère fédéral de l'Economie et de la Technologie tirait en janvier 2008 la conclusion suivante : «Le concept de 'ressource savoir' à la base des innombrables programmes de Wissensmanagement condamnés à l'échec s'est révélé trop étroit. C'est la raison pour laquelle il a mené à une impasse sous la forme d'un management du savoir excessif se manifestant par la mise en ouvre d'une gestion informatisée de bases de données détachées le plus souvent de tout contexte, générant des coûts de collecte, alimentation et entretien incommensurables en termes de budget, de gestion du temps comme de ressources humaines 》 (rubrique « Einführung von Wissensmanagement », consultée le 29-01-2008).

A ses débuts, le Wissensmanagement était donc un beau mythe fondé sur une vision beaucoup trop réductrice de la notion de savoir. Et quant à l'espoir de voir grâce à ses outils s'accroître dans l'économie la créativité, c'est-à-dire la capacité à innover, il s'est vite révélé erroné lui aussi. En effet, "la tentative de collecter, traiter et façonner le savoir existant de la manière la plus exhaustive possible mène souvent [à l'effet contraire] : elle inhibe la créativité, puisqu'elle accapare une grande partie des ressources humaines pour la gestion du savoir existant» (Howaldt, 2006).

Ce mythe répondait à une première phase dans l'approche de l'innovation, construite sur l'idée que le transfert des savoirs s'assimile au transfert des technologies : c'est-à-dire à la transmission des connaissances de la recherche fondamentale vers l'entreprise en vue de leur intégration dans un bien ou service à mettre sur le marché et, dans le sens inverse, à l'identification de la demande du marché - une information à faire 'remonter' vers la R\&D. Cette vision est elle aussi réductrice puisqu'elle ne couvre qu'un aspect de l'innovation: la R\&D, et la structuration des flux d'information qui lui sont inhérents. Elle n'en est pas moins importante, et les différentes contributions de cette journée d'étude ont décrit les efforts entrepris en Allemagne par les acteurs économiques et les pouvoirs publics pour améliorer encore la circulation de l'information en la matière. 
Le terme de Wissensmanagement intègre donc la question du transfert des technologies et des savoirs, de même que celle de leur management. Reste celle de l'acception à donner à la notion de 'savoir'. Il existe bien un terme prisé en France, et qui repose à première vue sur une acception large, à savoir « intelligence économique ». Mais ce terme, tout aussi connoté de magie que Wissensmanagement, est intraduisible en allemand, et pour cause.

Bien sûr, certains grands groupes allemands fortement internationalisés, cotés à New York, ont importé des méthodes de management américaines et quelques notions de «business intelligence ». Mais l'américanisme se contente de dorer de la magie du rêve américain une réalité fort banale, à savoir ce que fait d'instinct ou de manière explicite toute entreprise intelligente et soucieuse de recueillir l'information/savoir stratégique pour sa compétitivité : mener une veille concurrentielle, informationnelle, réglementaire, une analyse des risques pays ou autres... Et si l'américanisation des concepts apporte du prestige, elle introduit aussi, dans ces groupes exposés à la concurrence mondiale, une vision un tantinet plus guerrière de la concurrence que ne l'est l'approche allemande, tout du moins celle de l'écrasante majorité des entreprises, donc des PME.

Si on fait abstraction de ces glissements sémantiques qui restent l'exception, et si on considère le tissu des PME, on s'aperçoit alors que le concept français d'intelligence économique est foncièrement impropre pour décrire la réalité allemande. Il désigne en effet avant tout une politique publique et non pas l'action des acteurs économiques : une politique qui vient en complément de la politique d'innovation et de compétitivité globale de la France, et qui se décline du haut vers le bas - des pouvoirs publics vers les entreprises. Son approche top-down est double : du politique vers les acteurs économiques, et de la capitale vers l'échelon local. Dans ce dernier cas, elle se présente sous le terme "d'intelligence territoriale » qu'on peut définir, selon le directeur économique de l'Association des Chambres Françaises de Commerce et d'Industrie, comme «la déclinaison locale d'une stratégie nationale d'intelligence économique publique et privée à destination des PME, sur tout le territoire » (Clerc, 2007). Autrement dit : une approche nationale pour le site France, définie au sommet de l'Etat par un Haut responsable en charge de l'intelligence économique auprès du Premier ministre, est portée jusqu'aux PME au niveau local, grâce à un dispositif institutionnel de structure pyramidale s'appuyant entre autres sur le ministère de l'Intérieur. A l'échelon régional, elle prend par exemple pour relais les DRIRE afin de diffuser auprès des entreprises l'UtiLisation de l'Information Stratégique (ULIS) selon l'objectif suivant: "A l'image d'une démarche qualité, l'acquisition, l'exploitation, la diffusion et la protection d'informations ciblées (technologiques, réglementaires, commerciales, concurrentielles, ...) doit irriguer tous les métiers de l'entreprise », comme le formulait à la fin 2007 la DRIRE Nord Pas-de-Calais.

Une telle conception est totalement étrangère aux Allemands. La République Fédérale est un Etat fédéral où les politiques de compétitivité sont définies dans les Länder. La compétitivité globale du site Allemagne repose donc, au plan structurel 
institutionnel, sur le polycentrisme décisionnel et une approche de bottom-up. Il en va de même de la structure des activités, et donc de l'innovation, perçue dès lors comme un effort collectif, multi-niveaux et multidimensionnel, établi sur la confrontation ou la mise en concurrence des approches et modèles. Dans une culture fondée sur le principe de subsidiarité, aucun dispositif central n'est donc concevable pour impulser une dynamique de compétitivité ou d'innovation. En ce qui concerne la gouvernance des entreprises, les hiérarchies plates établies sur les compétences individuelles et le principe de collégialité sont contraires elles aussi à toute logique pyramidale. Et si, en matière d'innovation, l'attention des acteurs publics et privés se concentre en Allemagne comme en France sur la R\&D, outre-Rhin, on tend désormais vers une lecture plus large de l'innovation: "Seuls les Hommes sont innovants. La technologie n'est pas innovante, elle est tout au plus le fruit de l'innovation » (Putzhammer, 2004). Or ce sont les Hommes, non les institutions, qui portent le savoir...

S'ajoute à ces différences systémiques entre nos deux pays une différence fondamentale qui est, elle, d'ordre culturel; elle rend à elle seule incompatibles les deux approches. Le pivot en est l'approche de la concurrence. En France, l'intelligence économique est fondée sur un concept de "guerre économique», avec ses écoles propres ; mais cette approche est enseignée plus généralement dans toutes les écoles de management en France. Or pour un Allemand, l'idée même de considérer la concurrence, la compétition mondiale, comme une guerre relève de l'absurde. J'ai interrogé un jour sur ce point une spécialiste allemande des questions de gouvernance et des relations internationales, notamment dans les grandes entreprises, et elle m'a répondu ceci : "La concurrence, une guerre? Une absurdité dans la culture allemande où domine au contraire une approche partenariale de la concurrence. Le concurrent actuel est toujours dans le même temps un partenaire, ne serait-ce que potentiellement » (Reisach, 2007).

$\mathrm{Ce}$ constat vaut pour un Etat comme pour une entreprise. Le fait d'être concurrents en affaires, de s'affronter sur une cause ou un point précis n'exclut nullement qu'on se respecte et même qu'on s'apprécie. Pour le dire schématiquement : en allemand, concurrence implique le partenariat, avec la conclusion de compromis bénéfiques à toutes les parties; en France, concurrence est synonyme de guerre, et il n'y a généralement qu'un seul vainqueur. C'est la première idée, celle de partenariat, qu'on a vue déclinée sous la logique de réseaux, de complémentarité, et de mise en commun des compétences des uns et des autres. La formule d'intelligence économique, construite sur la logique du top-down, et établie sur la conception de la guerre économique, est donc foncièrement étrangère à un système économique fondé sur le principe de subsidiarité, c'est-àdire la responsabilité de chacun des acteurs et le partage des compétences. Dans l'approche allemande, le savoir n'est pas synonyme de pouvoir, il ne protège pas une fonction; il est au contraire considéré comme un 'bien' dont la diffusion génère de nouveaux savoirs et est en ce sens, puisqu'il est partagé, source d'innovation. 


\section{La créativité et la compétitivité naissent du partage des savoirs}

Cela étant, même pour les Allemands, le concept de Wissensmanagement est difficile à définir. Après la première phase évoquée ci-dessus où il se résumait à la gestion électronique de données, on définit aujourd'hui généralement comme une méthode de gestion des ressources humaines puisqu'au coeur de toute activité, il y a, comme l'a démontré M. Domdey à propos du cluster Biotech, d'abord des individus. Cela présuppose des entreprises considérées comme intelligentes, perçues comme des organisations apprenantes, ouvertes et transparentes, au cœur desquelles se trouve l'individu comme porteur des savoirs.

La fondation Steinbeis, qu'on admire beaucoup en France et à qui on prête volontiers toutes sortes d'activités obscures et qui n'est en réalité qu'un outil, comme tous les autres instituts, pour aider les process de management à se rénover, a défini ainsi l'acception clé sur laquelle repose le Wissensmanagement : "le savoir est bien plus que l'information; le savoir repose sur l'expérience et génère la capacité à agir. En ce sens, le savoir est foncièrement humain, et même le logiciel le plus élaboré de Wissensmanagement ne saurait le reproduire ».

Cette définition est la clé pour comprendre la compétitivité des PME allemandes, leur force d'innovation et aussi la compétitivité des clusters. Au sens large, elle implique que, l'individu étant porteur de savoirs, c'est le partage de ces savoirs qui va créer le savoir. Pour entrer plus profond encore dans la culture allemande, il faudrait même dire que ce partage est la clé de voûte du système d'innovation allemand. Or c'est aujourd'hui seulement (c'est ce que montre l'engouement pour le terme de Wissensmanagement) qu'on en prend toute la mesure, qu'on comprend réellement ce que cela signifie.

Les principales caractéristiques du système d'innovation allemand sont les suivantes : polycentrisme des affaires, des pouvoirs politiques, des administrations, des centres économiques, des établissements de recherche, des universités, des représentations de la société civile organisée. Ce polycentrisme implique la concurrence comme le principe de fonctionnement des marchés et comme un facteur favorable à la diversité des solutions proposées. Cette acception de la concurrence va bien au-delà de la pratique du benchmarking, fort développée au demeurant. Elle est fondatrice du modèle allemand de l'innovation. Elle repose sur l'idée que seule la confrontation d'approches multiples permet de générer l'excellence.

Troisième élément clé du système d'innovation allemand, corollaire de la diversité (polycentrisme) et de l'émulation (concurrence) des approches : l'échange. $\mathrm{Au}$ niveau macro-économique, il se traduit par exemple dans le fait que l'économie allemande est construite sur les échanges commerciaux. Or la notion d'échange est fondamentale, et même un élément fondateur de la culture allemande : qu'il s'agisse d'échange de savoirs entre individus ou via des réseaux de transfert. Dans une République fédérale marquée par le polycentrisme décisionnel, seule la circulation de l'information permet en effet d'assurer la cohérence d'un ensemble par nature disparate. La circulation des savoirs constitue le facteur de cohésion nationale par 
excellence. La presse généraliste, polycentrique elle aussi, est diverse, et elle a pour rôle de rendre accessible à tous le savoir permettant à chacun de prendre sa décision. La presse spécialisée a la même fonction dans le monde professionnel. Quant à l'outil Internet, il est par essence une plate-forme d'échanges ; un des axes de la politique allemande de compétitivité (ou d'innovation) a d'ailleurs consisté à inciter les PME à se raccorder au réseau et à s'équiper de tous les outils liés.

Enfin, et c'est un aspect qu'on oublie souvent quand on évoque les politiques d'innovation, le territoire allemand est étroitement maillé d'infrastructures assurant la mobilité des savoirs comme des personnes : réseau autoroutier, ferroviaire, etc. En Allemagne, les décisions se prennent partout : peu importe que le site de votre PME soit à Hambourg, à Munich ou à Berlin, puisque vous rencontrerez votre partenaire en affaires à Francfort, à Cologne ou à Leipzig. Les idées/savoirs sont aussi mobiles que les personnes. On comprend mieux, dès lors, l'importance des foires et salons professionnels qui contribuent eux aussi à ces échanges. Quant aux fédérations professionnelles, aux chambres de commerce ou aux syndicats comme aux fédérations patronales, ces corps intermédiaires exercent eux aussi une fonction de transfert des savoirs auprès de leurs adhérents. Outre-Rhin, la circulation de l'information est structurelle. Elle est omnidirectionnelle, c'est-à-dire à la fois horizontale, verticale et transversale. Et elle inclut la société civile organisée.

En un mot : elle est la manifestation corollaire d'une société régie par le principe de subsidiarité, c'est-à-dire reposant sur l'autonomie des acteurs et leur responsabilité, en l'occurrence celles des PME. Et, sur cet arrière-plan institutionnel et culturel, ce sont les entreprises ou les centres de recherche qui nouent eux-mêmes leurs coopérations et partenariats, fortes des compétences acquises et cultivées grâce à la mise en réseau et l'échange des savoirs. Ce n'est qu'ensuite que les parties prenantes s'adressent aux pouvoirs publics pour les inclure dans leurs réseaux et, le cas échéant, leur demander leur contribution : créer des conditions cadre favorables à l'activité.

A titre d'exemple de ces réseaux d'échange qui font la force d'innovation des entreprises allemandes, je citerai ici une institution appelée le RKW, le Centre de rationalisation et d'innovation de l'économie allemande (Rationalisierungs- und Innovationszentrum der Deutschen Wirtschaft). Le RKW est une sorte de tête de réseau venant en complément de réseaux préexistants ; son approche est elle aussi celle du bottom-up. Le RKW avait été créé en 1921, puis recréé après la guerre à l'initiative originelle des entreprises, des partenaires sociaux et des acteurs publics. Ce centre agit à deux niveaux. Puisqu'il dispose de l'information que lui transmettent les entreprises, il fait 'remonter' au niveau politique des préconisations pour que les pouvoirs publics agissent sur le cadre réglementaire afin de le rendre plus favorable à l'activité. Au niveau de ses adhérents, ce Centre agit sur la formation et le conseil pour aider les entreprises à se rationnaliser ou à se moderniser. On voit bien là, en considérant le type des acteurs représentés dans ses instances, que la société civile, et notamment les syndicats, donc les salariés, sont impliqués dans l'effort d'innovation.

Et on entrevoit que, dès lors, le Wissensmanagement s'assimile aujourd'hui principalement à une approche rénovée du management des ressources humaines 
dont le pivot est le respect du savoir, du savoir-faire et de l'expérience de l'individu. Car, et c'est là le coeur de l'acception large de l'innovation, telle qu'elle tend à prédominer aujourd'hui en Allemagne : le savoir n'est pas modélisable. Le savoir, c'est ce qui permet à l'individu d'agir en tant qu'être social - et donc en tant qu'acteur économique. On comprendra aisément la place éminente qui est dévolue dans cette GRH à la formation continue, en un mot : à la culture de ce savoir qui repose sur l'expérience humaine et qui, de ce fait, s'inscrit dans un contexte. En effet, " ce n'est que la sélection opérée dans [la] masse d'informations, la mise en perspective des divers éléments, leur interprétation qui, en en faisant jaillir le sens, créent le savoir. Le plus performant des logiciels ne saurait remplacer l'intelligence humaine, la capacité de jugement individuelle ni la volonté de travailler en partenariat. Le véritable patrimoine des entreprises est de nature humaine » (Reisach, 2007). Or, et c'est là un autre facteur clé systémique du système d'innovation allemand, "la performance, la créativité et l'innovation ne peuvent s'épanouir que dans un contexte professionnel serein et constructif, établi sur l'échange humain et donc le partenariat» (ibid.). Le Wissensmanagement signifie par essence un mode de gouvernance de l'entreprise qui valorise l'individu en tant que porteur des savoirs.

Pour conclure, l'intelligence de l'économie vient aux PME allemandes par tout un ensemble de facteurs qui sont avant tout culturels. Ils impliquent une attitude qui permet de saisir la globalisation comme une chance, comme une incitation permanente à innover pour gagner en compétitivité. L'intelligence se fonde alors sur une approche dynamique de processus où les compétences, les responsabilités, se trouvent à chaque niveau de l'entreprise. Cette dynamique est entretenue du fait que les PME allemandes forment $80 \%$ des apprentis et contribuent dès lors à créer le lien social. L'apprenti qui va travailler dans une PME est formé, socialisé par l'entreprise, et ainsi socialisé pour l'économie. Quoi de plus logique dès lors d'inclure dans cette formation l'aptitude à l'apprentissage - et de considérer l'entreprise elle aussi avant tout comme une organisation apprenante? «L'innovation naît dans un processus où l'économie, la science, la formation et la société agissent en réseau et dont le centre est l'individu en tant que porteur des savoirs " (ministère de l'Economie du BadeWurtemberg, www.baden-wuerttemberg.de, déc. 2005). C'est bien là une approche globale où un rôle clé revient aux PME, que leur réactivité rend foncièrement compétitives, d'autant que cette réactivité est décuplée par une intense circulation des savoirs au sein de réseaux associant tous les acteurs publics et privés impliqués dans l'innovation. Et, pour ces PME, " la meilleure politique de soutien qui soit se résume à une bonne politique économique tout court», ainsi que l'a formulé un jour l'Arbeitsgemeinschaft Mittelstand, le lobby de huit fédérations professionnelles rassemblant plus de la moitié des PME allemandes.

LE Wissensmanagement Ne SIGNIFIE RIEN D'AUTRE, au fond, que de rendre plus efficiente encore la circulation des savoirs. Il suffit pour cela que les pouvoirs publics accentuent la politique d'offre telle qu'ils la mènent, c'est-à-dire une politique où le Bund ou le gouvernement d'un Land se contente d'offrir la possibilité 
aux acteurs de mieux travailler ensemble, que les moyens de cette politique soient budgétaires ou qu'il s'agisse de l'instauration de portails favorisant l'échange. Mais la priorité absolue des pouvoirs publics comme des acteurs économiques est le développement des compétences, une autre considération de la ressource humaine, de l'individu comme porteur de savoirs. Or cela implique à terme une politique éducative et de formation totalement différente, centrée plus encore sur le lifelong learning et la capacité à faire fructifier les savoirs explicites, acquis, mais aussi implicites. Il suffit, au fond, de miser sur une plus grande flexibilité des savoirs individuels. Car la culture de l'innovation est un des traits systémiques de l'Allemagne. Elle repose sur une logique contractuelle d'échanges entre les acteurs à la base, elle-même construite sur la confiance mutuelle. En ce sens, le Wissensmanagement est bel et bien une manifestation de l'intelligence économique des PME allemandes qui, grâce au développement de leurs réseaux de partenariats où se partagent les savoirs, se dotent chacune de la capacité à lire intelligemment l'évolution de leur contexte économique et à appréhender leurs marchés en toute connaissance de cause et en pleine autonomie. On voit bien là sur quoi repose l'approche globale de l'innovation qui a cours outre-Rhin: sur une vision de l'individu, de l'entreprise, du cluster, dans leur environnement et dans leur interaction globale.

\section{Indications bibliographiques}

BOURGEOIS I. (dir.), Allemagne : compétitivité et dynamiques territoriales, CIRAC, Cergy-Pontoise, 2007

BOURGEOIS I., «Comment l'intelligence vient aux PME allemande», Regards sur l'économie allemande, $\mathrm{n}^{\circ} 74$, décembre 2005 , p. 29-36

CLERC P., «L'intelligence territoriale: l'originalité de la France», Le Monde, Supplément «Les cahiers de la compétitivité. Intelligence économique (publicité)», 13 juin 2007, p. [n.c.]

«Culture d'entreprise : ,le piège de l'américanisation'. Un entretien avec Ulrike REISACH», Regards sur l'économie allemande, ${ }^{\circ} 84$, décembre 2007, p. 21-28

HOWALDT J., «Eine ernüchternde Bestandaufnahme. Mythos Wissensmanagement», Dossier «Wissen in Unternehmen», Journal Arbeit, vol. 1, printemps 2006, p. [n.c.]

STEINBEIS-TRANSFERZENTRUM WISSENMANAGEMENT \& KOMMUNIKATION, Mensch \& Wissensmanagement [en ligne] : www.steinbeis-wissensmanagement.de/Verstaendnis/Mensch [consulté le 07/01/10]

PUTZHAMMER H., «Plädoyer für einen erweiterten Innovationsbegriff», in «Innovation - was geht das die Gewerkschaften an?», Materialien und Argumente [en ligne], $\mathrm{n}^{\circ}$ 1, juin 2004, p. 3-7: http://www.dgb.de/themen/innovation/grundsatzpos.htm/ [consulté le 07/01/10]

DRIRE NORD PAS DE CALAIS, ULIS. Appel à projets pour l'UtiLisation de l'Information Stratégique. Clôture des dossiers le 15 septembre 2007 [en ligne] : www.nord-pas-de-calais.drire.gouv.fr/di/ulis/ulis.htm, dossier consulté le 18-01-2008

RKW RATIONALISIERUNGS- UND INNOVATIONSZENTRUM DER DEUTSCHEN WIRTSCHAFT E.V., $R K W$ Kompetenz-Zentrum, [en ligne] : www.rkw.de [consulté le 07/01/10]

BUNDESMINISTERIUM FÜR WIRTSCHAFT UND TECHNOLOGIE (BMWI), Wissensmanagement als Innovationsmotor [en ligne] : www.wissenmanagen.net [consulté le 07/01/10]. 
$-\mathbf{V}$ -

Mise EN PERSPECTIVE EUROPÉENNE 



\title{
Politique de clusters : des expériences de gouvernance politique
}

\author{
Dieter REHFELD
}

"La promotion des clusters ne peut être une fin en soi ; elle n'a de sens politique que si, au-delà du soutien direct, émerge une valeur ajoutée ". Dieter Rehfeld, chercheur à I'Institut du Travail et de la Technique (IAT) de Gelsenkirchen, revient dans sa contribution sur la finalité de la gouvernance et de la politique des clusters, évolutives par essence. Adoptant une démarche prospective, il plaide en faveur de clusters certes créatifs, mais également ouverts sur l'extérieur, pour éviter tout effet de "verrouillage " des savoirs et des compétences.

\section{La politique de clusters: une orientation nouvelle dans un contexte de mondialisation}

La politique de clusters est aujourd'hui une composante reconnue de la politique structurelle. Elle intervient à tous les niveaux décisionnels et sous différentes formes, à l'aide d'instruments divers. Il y a environ 15 ans, a démarré un processus étonnant, au fil duquel la politique de clusters s'est imposée dans un mouvement plutôt ascendant ("bottom-up »), jusqu'à être aujourd'hui l'objet de la politique aux différents échelons.

Le succès de la politique de clusters tient notamment au fait que cette approche promet une consolidation stratégique de la politique économique et structurelle dans les économies capitalistes. L'innovation, la compétence et la mise en réseau en sont les notions clés, offrant une alternative à la concurrence mondiale sur les prix et les coûts, une concurrence à laquelle il est impossible de faire face. Les présupposés communs de la politique de clusters peuvent se formuler comme suit :

- dans l'économie de demain, fondée sur le savoir et interconnectée à l'échelle mondiale, les clusters constituent les pôles d'innovation d'une économie nationale ;

- le potentiel de formation de clusters ou de synergies possibles au sein de clusters est loin d'être épuisé ;

- le politique peut activer ce potentiel. Pour ce faire, il faut de nouveaux instruments et stratégies, qui peuvent aujourd'hui puiser dans de nombreuses expériences internationales couronnées de succès ;

- ces éléments font de la promotion des clusters la stratégie centrale de modernisation durable d'une économie : à l'échelle européenne, elle constitue l'un des piliers de la mise en œuvre de la stratégie de Lisbonne. 
On pourrait discuter longuement du contenu empirique de ces présupposés, les analyses scientifiques réalisées sur le développement et l'importance des clusters pour une économie nationale ne menant absolument pas à des résultats convergents. Les données statistiques disponibles rendent de moins en moins compte des changements et des interdépendances économiques; les clusters se trouvent dans des phases de développement très différentes; ils peuvent tout à fait, justement dans les périodes de crises économiques, générer aussi des processus de contraction cumulatifs, comme l'a montré l'évolution dans les régions houillères et sidérurgiques traditionnelles. Enfin, les différents clusters se sont constitués de manières très diverses, et ne sont donc que partiellement comparables (sur l'état de la recherche, voir Asheim/Cooke/Martin, 2006).

Mais notre propos n'est pas d'analyser ici le contenu empirique des présupposés cités (pour une analyse détaillée, voir Rehfeld/Terstriep, 2007). Nous nous concentrerons plutôt sur les expériences et perspectives du pilotage politique et des structures de gouvernance du développement de clusters. Le mot " cluster " revêtant des significations très diverses, il semble nécessaire de définir les principaux termes de ce débat (voir Rehfeld, 1999) :

- on entend par cluster ou développement de clusters des structures ou processus économiques autonomes entraînant, du fait de l'attractivité de sites, une concentration géographique d'entreprises ;

- la politique de clusters englobe des approches stratégiques dans des domaines politiques parfois très différents, qui constituent le cadre de la promotion des clusters. La politique des clusters ainsi définie intervient aux niveaux européen et national ; mais en Allemagne, elle est surtout du ressort des Länder; on peut donc parler d'approche descendante (top-down);

- la gouvernance des clusters renvoie enfin à des activités régionales issues de la coopération (ou mise en réseau) d'acteurs publics et privés d'un ou de plusieurs clusters sur le terrain, ou bien destinées à soutenir ceux-ci dans leur développement ultérieur; on peut donc parler d'approche ascendante (bottom-up).

\section{Le développement de clusters : quelques postulats de base}

La promotion des clusters ne peut être une fin en soi ; elle n'a de sens politique que si, au-delà du soutien direct, émerge une valeur ajoutée. C'est notamment le cas lorsque les moyens financiers initialement légitimes pour des régions ayant des problèmes structurels sont ensuite dépensés pour une politique de « renforcement des forces », donc s'ils sont prioritairement déployés dans les régions déjà structurellement fortes.

$\mathrm{Si}$ des conceptions très diverses du cluster se sont développées, il est toutefois possible d'identifier des composantes communes (voir schéma 1). Les clusters impliquent la concentration géographique des fonctions économiques. 
A la différence des agglomérations, ils ont un lien technologique ou sectoriel, la chaîne de valeur. La proximité géographique crée des avantages résultant d'infrastructures communes, de la présence sur un même site de fournisseurs et d'organismes de recherche spécialisés ainsi que de la possibilité d'une communication directe et même souvent informelle. A partir d'une certaine masse critique d'activités, difficile à définir a priori, et d'une certaine densité, des effets positifs (synergies) sont possibles si les acteurs opèrent non pas de manière juxtaposée, mais en interconnexion. De telles interactions ne se produisent pas automatiquement (c'est là qu'interviennent la gouvernance et la politique des clusters) ; elles supposent un partage du travail et des savoirs, et donc une complémentarité des compétences des acteurs. Les interactions au sein d'un cluster sont toujours un équilibre entre coopération ou mise en réseau d'une part et concurrence d'autre part.

\section{Schéma 1 : Caractéristiques fondamentales de la notion de cluster}

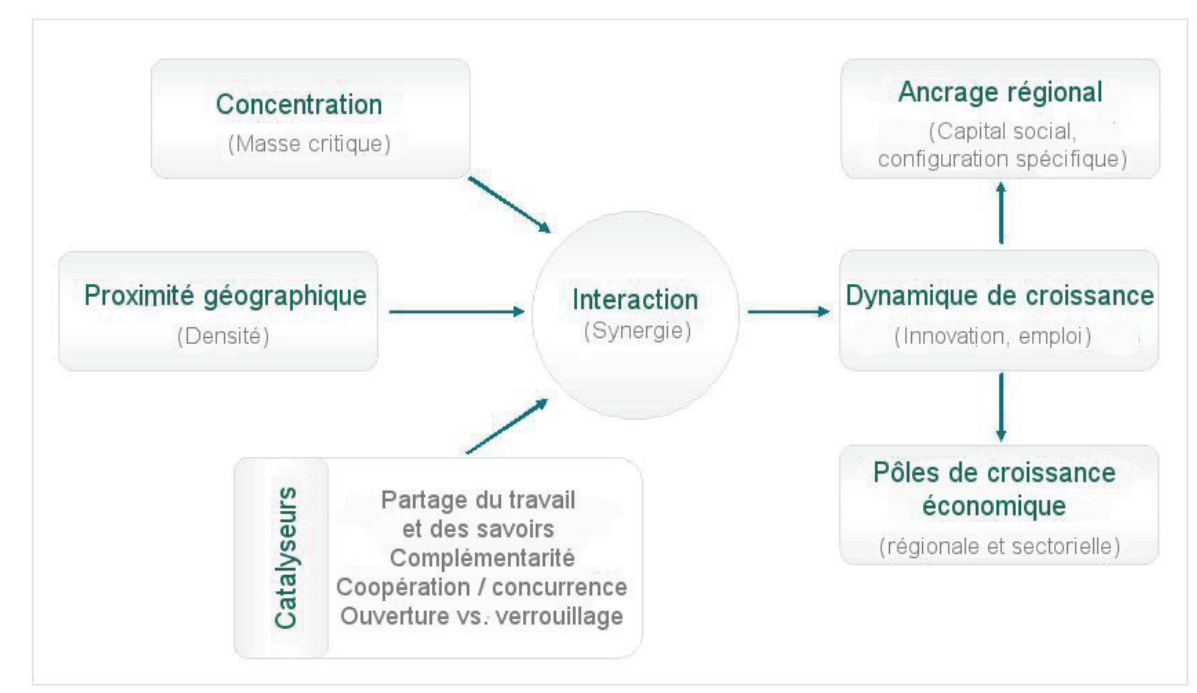

Source : (C) 2007 IAT - présentation de l'auteur.

On attend de ces clusters des effets positifs pour l'économie de la région et pour l'économie en général (c'est la finalité de la gouvernance et de la politique des clusters).

$\mathrm{Au}$ niveau régional, les clusters peuvent favoriser un ancrage territorial des entreprises ainsi que l'implantation d'autres entreprises. Une configuration territoriale offre en effet des avantages matériels et immatériels en termes de compétitivité (capital social notamment) qui, malgré des coûts des facteurs éventuellement assez élevés, rendent ce site attractif et le positionnent durablement dans la concurrence mondiale. Les clusters permettent d'escompter une dynamique 
de croissance fondée sur l'innovation, avec des effets positifs en termes d'emploi. Ces pôles ont donc un rôle de centres d'innovation, à la fois pour l'économie régionale et pour l'économie dans son ensemble, leur dynamisme ayant également des répercussions positives sur d'autres chaînes de valeur et d'autres régions (effets de débordement).

La politique de clusters ne peut produire de tels effets directement: ses possibilités d'action sont seulement indirectes (schéma 2).

Schéma 2 : Politique des clusters : modèle de gouvernance simplifié

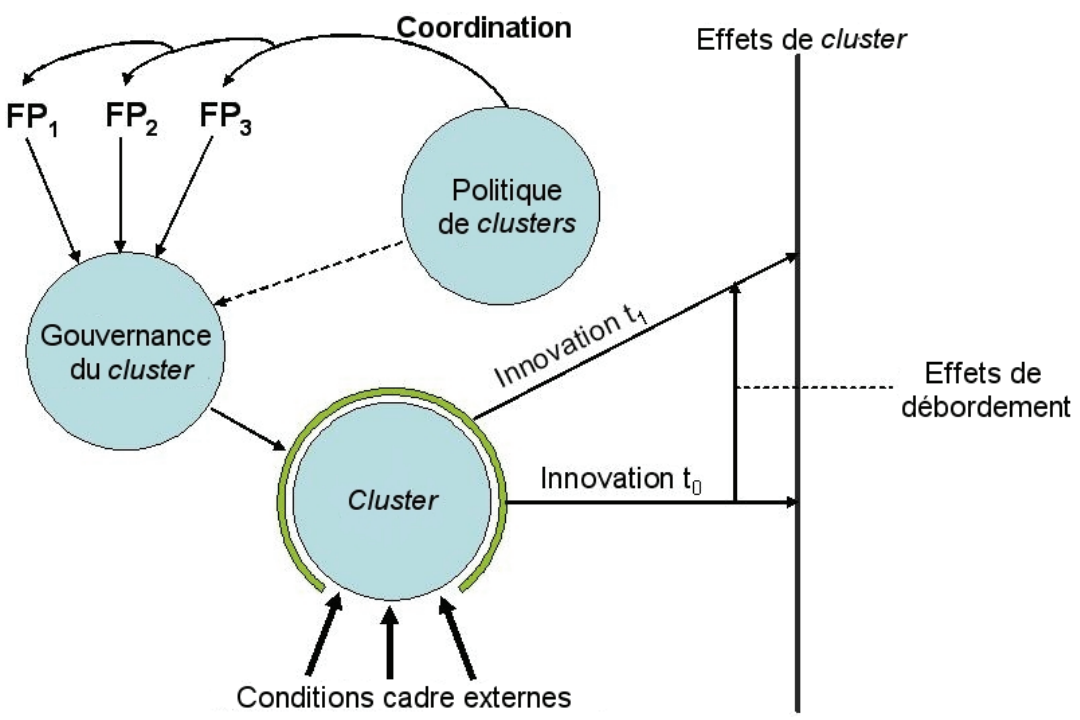

Source : C 2007 IAT - présentation de l'auteur.

Premièrement, la politique de clusters n'intervient pas de manière autonome, mais dans le cadre d'autres politiques. Pour être efficace, il lui faut échapper aux critères de référence de ces politiques: lorsqu'une politique de clusters est menée dans le cadre de la politique structurelle régionale, elle ne peut pour autant soutenir exclusivement les régions confrontées à des problèmes structurels. Lorsqu'elle intervient dans le cadre d'une politique de recherche et de technologie, il faut une conception de l'innovation dépassant les considérations purement techniques. Dans le cadre d'une politique de développement territorial, le souci dépassera le seul soutien aux agglomérations ou aux métropoles. La politique de clusters suppose donc une aptitude à coordonner des politiques spécialisées.

Deuxièmement, la politique de clusters ne peut fonctionner sans une gestion adaptée au niveau régional. Celle-ci va au-delà de la simple prestation de service pour les entreprises membres; la gouvernance de cluster a généralement la fonction d'instance de mise en ouvre de la politique de clusters. Ce 
processus n'est ni évident ni dépourvu de tensions, mais nous ne pouvons qu'y faire allusion ici (voir NICE, 2008).

Troisièmement, la gouvernance des clusters ne peut avoir qu'une action limitée sur le développement de clusters. Ce dernier est surtout influencé par le contexte général : concurrence mondiale, institutions du système national d'innovation et de production, conjoncture des différents secteurs, etc. La gouvernance peut contribuer à améliorer ces conditions extérieures, pour qu'il soit plus facile d'y faire face que sur d'autres sites, mais elle ne saurait en elle-même modifier les conditions cadre.

\section{La gouvernance des clusters : l'histoire d'un succès et ses promoteurs}

Les clusters ne sont pas un phénomène nouveau. Les économistes classiques des $\mathrm{XVIII}^{\mathrm{e}}$ et $\mathrm{XIX}^{\mathrm{e}}$ siècles ont déjà abordé la dimension géographique des activités économiques, et, à la fin du XIX $\mathrm{XI}^{\mathrm{e}}$ siècle, Alfred Marshall a étudié de manière détaillée les districts industriels (voir la synthèse dans Scheuplein, 2006). Mais on ne s'est guère demandé pourquoi les clusters ont largement disparu des débats politiques et scientifiques au $\mathrm{XX}^{\mathrm{e}}$ siècle, et n'ont plus eu le vent en poupe avant la fin $\mathrm{du} \mathrm{XX}^{\mathrm{e}}$ siècle.

Si l'on considère le débat actuel sur les clusters non comme une mode passagère, mais comme l'expression d'une mutation des structures et des stratégies économiques, il faut se mettre en quête d'explications. Le point de vue de Krugman (1991), qui invoque la quasi suppression des coûts de transport et de communication incitant les entreprises à chercher le site optimal - donc le principal cluster (ou, chez Krugmann, l'agglomération) pour la chaîne de valeur concernée - n'est pas convaincant puisque cet argument peut aussi déboucher sur la conclusion inverse, à savoir la recherche permanente des meilleures conditions territoriales et donc une diminution du poids de l'ancrage régional (pour une analyse détaillée de cette question, Rehfeld, 2001).

Les analyses se penchant sur ce qui vient après le fordisme sont plus intéressantes. Il s'agirait de montrer qu'il n'y a guère d'intérêt à constituer des clusters dans un système de production fortement standardisé, mais que cela a davantage de sens dans un système de production souple, caractérisé par un haut degré d'incertitude et des contraintes de flexibilité. Cela ne veut pas dire que les entreprises aient absolument besoin de constituer des clusters, mais que l'implantation au sein de tels pôles offre la possibilité de réduire les incertitudes et de faire face aux contraintes de flexibilité (voir Piore/Sabel, 1984).

Le début du récent boom des clusters a surtout été marqué par les analyses de Porter sur l'avantage concurrentiel des nations (1990). Le tableau 1 regroupe les principales étapes d'évolution survenues au cours des deux dernières décennies. En Europe, le cluster automobile de Styrie (Autriche) fut sûrement l'un des premiers projets de pilotage d'un cluster perçu comme suprarégional. L'évolution s'est ensuite accélérée, et l'on peut aujourd'hui considérer la gou- 
vernance de clusters comme une dimension bien établie d'une politique structurelle décentralisée.

En matière de recherche et d'expertise, la première conférence internationale consacrée aux clusters, organisée à Mexico en 1997 par la Banque mondiale, a constitué une étape importante.

A la suite de cette conférence, le Competitiveness Institute (TCI) a été fondé, dans l'esprit des analyses de Porter, débouchant sur le Cluster Whitebook (2003) et le Cluster Greenbook (2004), qui présentent le savoir international sur la gouvernance des clusters.

En Europe, la politique des clusters a reçu une impulsion cruciale dans le cadre de la stratégie de Lisbonne. Les programmes de la Commission, PAXIS, Pro Inno et Innova ont offert et continuent d'offrir aux régions à la pointe de l'innovation une plateforme leur permettant de développer et d'expérimenter de nouveaux instruments, mais également de se documenter et d'échanger sur les bonnes pratiques. Aujourd'hui, le développement de clusters est également bien ancré dans les institutions (Comité des régions en particulier) et les instruments (FEDER) de la politique régionale.

Tableau 1 : Politique de clusters : étapes d'une histoire à succès

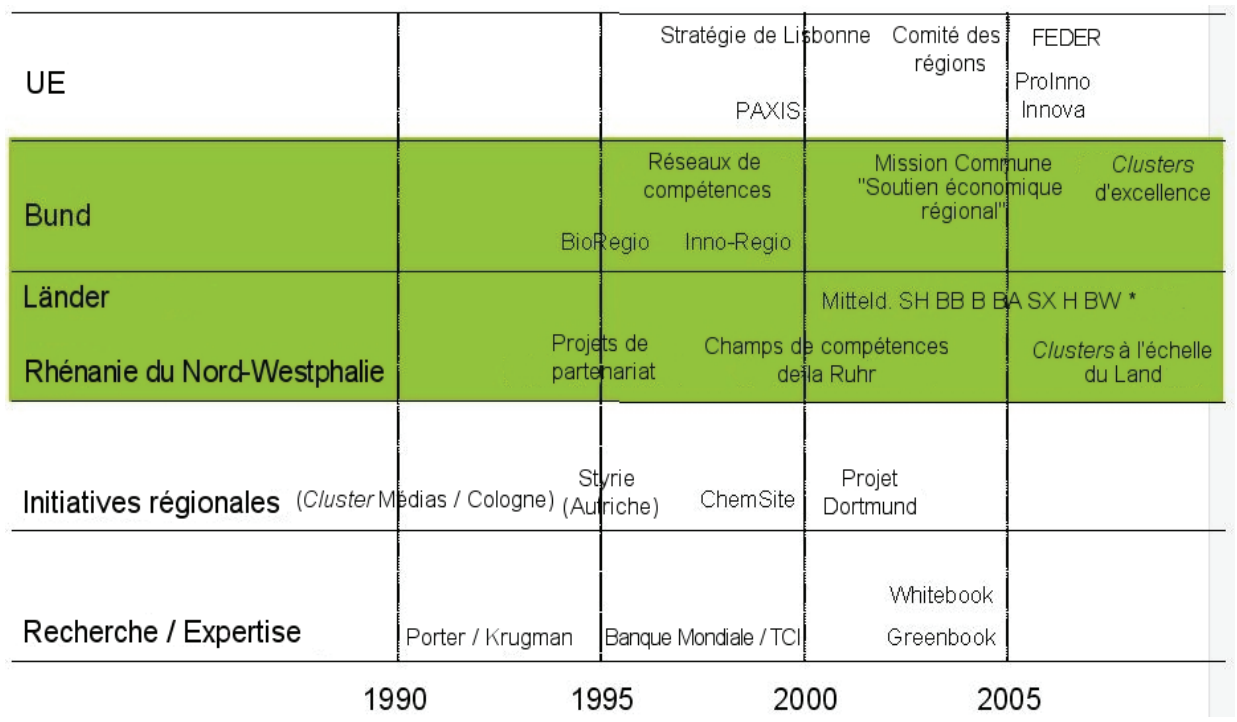

* Mitteldeutschland, Schleswig-Holstein, Brandebourg, Berlin, Bavière, Saxe, Hesse, Bade-Wurtemberg.

Source : (C) 2007 IAT - présentation de l'auteur.

La politique de clusters, mise en œuvre par les Etats nationaux ou, dans des Etats fédéraux comme l'Allemagne, par les Länder, s'est inscrite tardivement dans un champ préstructuré par des initiatives de terrain et par une plateforme 
européenne régie par des normes. En Allemagne, il existait déjà, il est vrai, depuis le milieu des années 1990 des activités reposant sur des idées comme celles de " renforcement des forces » ou de " réseaux régionaux d'innovation ", centrales dans la politique de clusters. Mais une telle politique, explicite et organisée à plus grande échelle, ne démarre en Allemagne (comme en France) qu'à partir de 2000, et s'accélère à partir de 2005.

\section{La politique de clusters : une gouvernance à plusieurs niveaux}

La politique de clusters est donc une gouvernance à plusieurs niveaux, présentant les caractéristiques suivantes (voir les contributions dans Tömmel (éd.), 2007, Benz et al. (éd.), 2007) :

- ce sont des modèles complexes d'institutions et d'acteurs ;

- les frontières entre les niveaux et les acteurs sont floues, de sorte que les responsabilités ne sont pas toujours clairement établies ;

- aucun acteur ne peut à lui seul parvenir au résultat souhaité ; les acteurs sont interdépendants ;

- la définition des objectifs et leur réalisation se font en interaction entre des acteurs publics et privés, dans un processus d'autorégulation plus que de pilotage classique par l'Etat ;

- de nouvelles techniques de pilotage apparaissent, qui ne font pas appel à des instruments d'autorité (et ne peuvent le faire).

Les débats politico-scientifiques sur les nouveaux modèles de gouvernance, au sein de l'Union européenne en particulier, peuvent semer le doute quant au succès de la politique de clusters : la gouvernance à plusieurs niveaux ne fonctionnerait en effet qu' «à l'ombre de la hiérarchie», lorsque l'État a la possibilité d'appliquer de nouveaux instruments d'autorité en cas d'échec de l'autoorganisation.

Or, ce modèle est justement impossible en matière de politique des clusters, celle-ci n'étant réaliste sans la participation active (y compris, de plus en plus souvent, financière) des acteurs. Pour comprendre les contraintes de cette politique, il est bon d'en avoir les grandes caractéristiques à l'esprit ; quatre aspects doivent être soulignés.

1) La politique de clusters vise à susciter l'auto-organisation, en proposant généralement un financement de démarrage comportant des aides publiques dégressives. Parallèlement, cette politique vise la production de biens collectifs qui n'ont pas été produits auparavant par les acteurs privés (dans le cas contraire, la politique de clusters ne serait pas nécessaire). Son propos n'est donc pas uniquement d'activer des potentiels latents jusque-là, mais aussi de modifier le comportement des entreprises : l'entrepreneur idéal n'est plus un combattant solitaire, mais une personne socialement responsable et économiquement en réseau. Notons en passant qu'il ne s'agit 
pas là d'une évolution touchant un domaine politique spécifique, mais qu'une telle orientation collective de l'activité entrepreneuriale est diamétralement opposée au droit de la concurrence, qui postule des acteurs isolés, opérant uniquement par l'intermédiaire du marché, et soupçonne dans la communication directe des ententes illicites.

2) Pour être efficace, la politique de clusters doit sélectionner, prendre des décisions de répartition. D'un point de vue économique, les clusters constituent des exceptions et non la règle; la politique de clusters est donc nécessairement une politique de redistribution. Pour compliquer les choses, les données statistiques permettant d'identifier et de distinguer clairement les clusters sont extrêmement limitées ; il règne par ailleurs une grande incertitude sur les évolutions à venir et donc sur les chaînes de valeur et les territoires, amenés à avoir un rôle central économiquement ; enfin, il n'y a aucun consensus sur les conditions qui doivent être réunies pour assurer le succès d'une politique de clusters. Il n'est donc pas surprenant que les champs thématiques de cette politique soient de plus en plus larges et semblables à l'échelle européenne, que le nombre de projets de clusters bénéficiant d'un soutien public soit de plus en plus important et que, parallèlement, les conditions favorables au développement de clusters soient de plus en plus floues, comme le montre déjà ce vocable élastique (minicluster, micro-cluster, cluster potentiel, éléments de cluster, etc.).

3) La politique de clusters est transversale, et dispose rarement de ressources propres. Un pan essentiel de cette politique en Europe, y compris et surtout en Allemagne, s'inscrit dans le cadre de la politique structurelle régionale. Or, celle-ci est axée sur la cohésion et sur l'équité économique et sociale, donc sur les régions qui, précisément, ne sont pas les plus fortes économiquement. La politique de clusters doit donc, comme il a été dit dans la deuxième thèse, tirer sa légitimité d'une valeur ajoutée économique.

4) La politique de clusters ne peut s'en tenir aux limites administratives : pour fonctionner, il lui faut dépasser largement la logique politique classique de territoires d'action clairement circonscrits. Les interdépendances économiques ne se superposent pas aux territoires politico-administratifs ; la politique de clusters requiert donc non seulement la coopération verticale des différents niveaux décisionnels, mais aussi la coopération horizontale d'entités politiques juxtaposées.

\section{La politique de clusters : un champ d'expérience et d'apprentissage}

La politique de clusters est confrontée à une telle multiplicité de défis qu'il lui faut pouvoir faire évoluer ses stratégies et ses instruments. Ce processus d'apprentissage se compose d'éléments tout à fait identifiables, et une répar- 
tition des tâches se dessine au sein du système européen de gouvernance à plusieurs niveaux (schéma 3).

A l'échelon européen correspond le champ expérimental. Les programmes cités plus haut (PAXIS, INNOVA et PRO INNO) mutualisent surtout des expériences émanant de la gouvernance de clusters établis. Ce cadre permet d'expérimenter de nouveaux outils et instruments, d'élaborer des normes destinées à professionnaliser la gestion des clusters et, enfin, de constituer des bases d'informations permettant de comparer et d'évaluer le développement de clusters. La coopération entre clusters et la mise en réseau fonctionnelle entre leur direction et les autres acteurs de la politique structurelle (issus principalement des domaines du soutien à la création d'entreprises et à la technologie, l'interaction avec les acteurs de la politique d'emploi et de formation étant encore très insuffisante), ainsi que la focalisation sur des problèmes sociaux urgents tels que l'efficacité énergétique et la gestion des ressources ou sur les défis des évolutions démographiques et sociales constituent des éléments cruciaux.

Les sociétés de conseil, les réseaux de directeurs de clusters, les organismes de formation continue impliqués dans les activités et, enfin, les grands congrès internationaux jouent le rôle de multiplicateurs. Si le paysage européen de la gouvernance de clusters reste très hétérogène, des normes de professionnalisme se sont toutefois imposées qui, à long terme, ne pourront être ignorées. Il faut toutefois noter que l'évaluation de cette activité est en encore à ses balbutiements et qu'il y a beaucoup à faire dans ce domaine.

Schéma 3 : Grandes lignes d'une division du travail dans un système européen de gouvernance à plusieurs niveaux

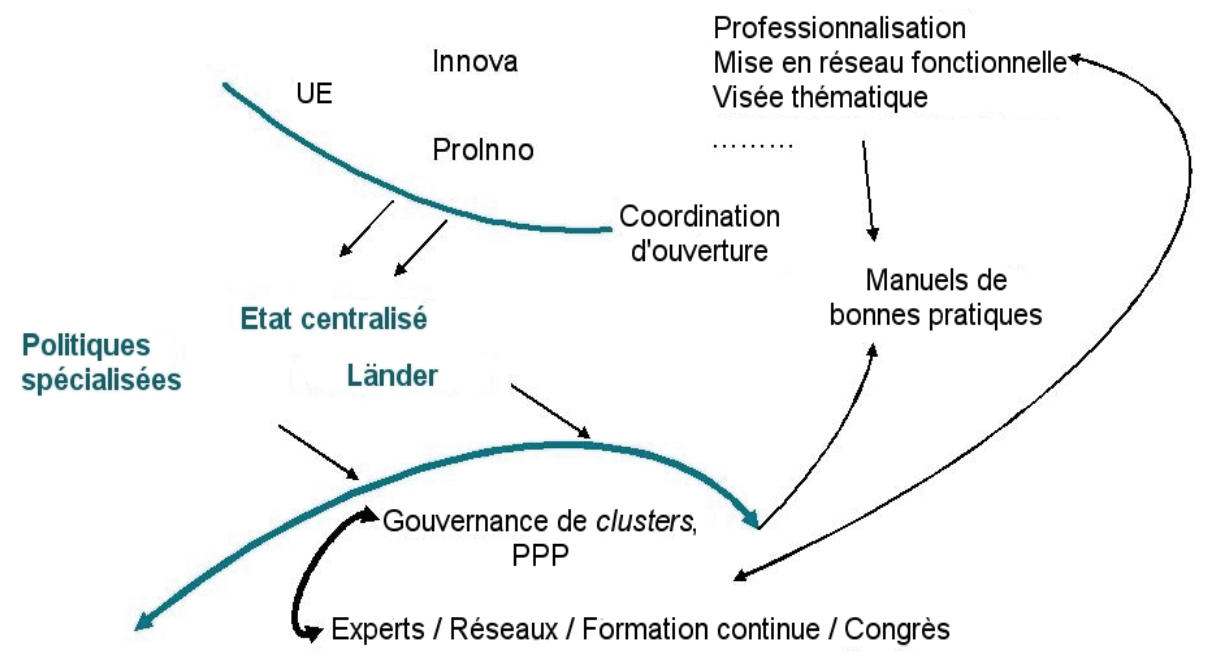

Source : (C) 2007 IAT - présentation de l'auteur. 
La politique de clusters menée par des Etats centralisés, ou par des Länder dans les Etats fédéraux, doit encore trouver sa propre place. Si nous reprenons les catégories clés du débat sur la gouvernance (pilotage par le marché, la hiérarchie et les réseaux comme points de référence), ce triangle (schéma 4) couvre pratiquement tous les domaines possibles. C'est la démarche des Etats et des régions confrontés à d'importants problèmes de restructuration (par exemple dans les pays d'Europe centrale et de l'Est ou dans le Brandebourg) ou celle des Etats à forte tradition de planification comme la France qui s'apparente le plus au pilotage classique. La difficulté majeure consiste dans de tels cas à associer les tendances centralisatrices des Etats au lancement d'une autoorganisation sociale.

\section{Schéma 4 : Modes de gouvernance dans la politique de clusters}

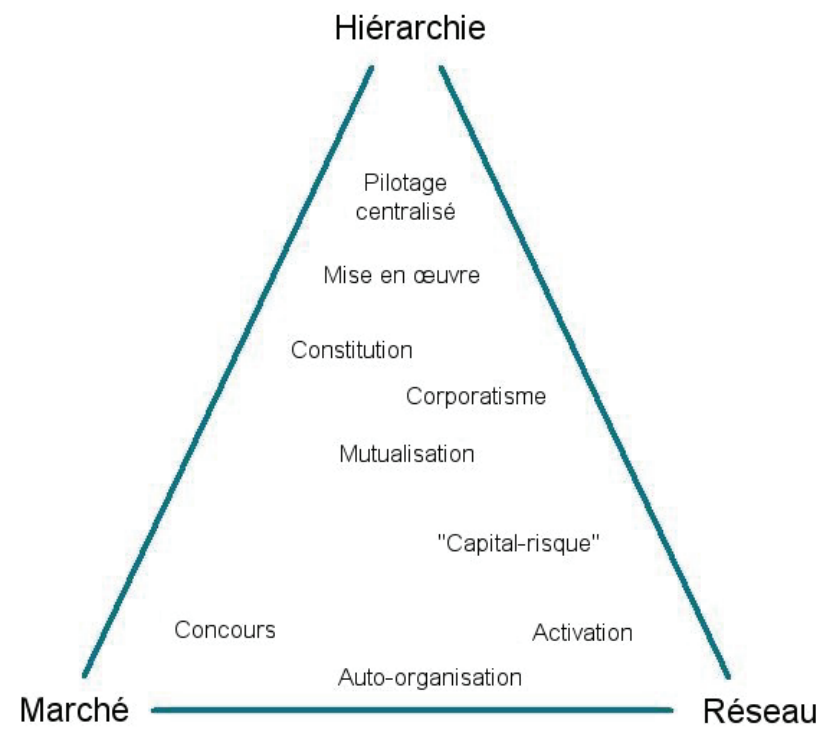

Source : (C) 2007 IAT - présentation de l'auteur.

On trouve à l'autre bout du spectre - et c'est un aspect souvent ignoré - des activités relevant de l'auto-organisation régionale d'entreprises qui ne font pas appel au soutien de l'Etat et ne le souhaitent souvent pas, préférant poursuivre leurs objectifs indépendamment de contraintes politiques et de l'attention des pouvoirs publics.

On trouve entre ces deux pôles toutes les formes hybrides possibles : les aides au démarrage, avec l'objectif d'une auto-organisation autonome et viable à terme, de même que le soutien à des projets de clusters d'excellence lauréats de concours de promotion, s'inscrivent encore du côté du marché et des réseaux. La mutualisation ou la constitution des ressources nationales via une forte coopération technologique ou une coopération comparable au corporatisme classique entre 
l'Etat, les syndicats et les entreprises sont plus proches des intérêts publics. Ces activités devront veiller à ne pas s'associer à une échelle trop restreinte, mais à préserver des possibilités d'ouverture vers l'extérieur, donc à rester ouvertes à de nouvelles impulsions.

Il existera toujours des modèles très différents, en fonction de la situation économique de départ, de la disposition des acteurs sociaux à collaborer à la production de biens collectifs, ou bien des philosophies de la gouvernance politique et administrative et des systèmes de régulation nationaux. Tous ces modèles ont toutefois un point commun : chacun d'eux requiert, à partir d'un certain moment, la participation active des acteurs, en particulier celle des entreprises. Ils sont en outre tributaires d'une concentration des ressources publiques qui devraient largement cibler les activités auto-organisées ou considérées comme stratégiques d'un point de vue politique.

Quel que soit le point de départ, la politique de clusters devra - pour la raison précise qu'on ne peut faire aucune prévision claire quant à l'évolution économique à venir - trouver un autre moyen de résoudre les problèmes de répartition évoqués. La voie actuellement la plus prometteuse consiste à organiser cette politique d'une manière analogue au financement par le capital-risque :

- dans un premier temps, il est tout à fait judicieux de soutenir un large éventail de projets, tout en ayant conscience que tous ne seront pas couronnés de succès ;

- il s'agit ensuite de lier (financièrement et matériellement) les aides à des objectifs clairs et d'organiser un appui à la réalisation de ces objectifs ;

- il faut enfin, à un moment donné, décider quels projets peuvent fonctionner de manière autonome, en portant les fruits sociaux escomptés, lesquels ont encore besoin de soutien car les objectifs souhaités requièrent plus de temps que prévu et quelles activités publiques doivent prendre fin.

Sans ce rapport à l'auto-organisation, les activités de pilotage de clusters, créées du bas vers le haut, et celles de l'Etat centralisateur entreront en conflit. Dans le pire des cas, non seulement les moyens publics seront gaspillés faute d'efficacité, mais la dispersion des ressources publiques pourra même contrecarrer les processus autonomes de développement de clusters. Nous pouvons illustrer brièvement ce danger en prenant l'exemple des biotechnologies : pour fonctionner, les clusters de ce secteur ont besoin d'infrastructures spécifiques (laboratoires, niveaux de sécurité), d'un environnement scientifique très large, adéquat, de compétences spécialisées en matière de financement par le capital-risque, de fournisseurs spécialisés pour les matériels, équipements, etc. et, enfin, de main-d'œuvre hautement qualifiée. Imaginons, dans la perspective d'une politique de clusters très large, que chaque site universitaire doive être développé afin de comporter toutes les fonctions mentionnées ; en cas de doute, c'est exactement l'effet contraire qui se produira : aucun de ces sites n'atteindra la masse critique nécessaire pour mettre en place l'environnement adapté au bon développement d'un cluster. 


\section{La politique de clusters en période de crise économique mondiale}

Les présentes considérations montrent que la politique de clusters est confrontée à des défis importants. On ne peut la mesurer uniquement à l'aune de son aptitude à accroître la capacité d'innovation de la société. La conception et l'implémentation de nouvelles stratégies et de nouveaux instruments de mise en œuvre de cette politique constituent en elles-mêmes une innovation sociale impliquant un profond changement de structures politiques, administratives et sociales. Trois grandes dimensions sont en jeu : une conception nouvelle des stratégies et des instruments politiques ; la mise en place de structures décentralisées (gouvernance de clusters) afin d'utiliser efficacement ces instruments; et une réorientation stratégique des entreprises afin de trouver un nouvel équilibre entre orientation territoriale et globale.

Ces dernières années, la politique de clusters a pu se développer dans des conditions économiques favorables et poser des bases essentielles pour faire face à ces nouveaux défis. Dans le contexte de la crise économique mondiale actuelle, déclenchée par l'effondrement des marchés financiers, avec des conséquences à venir sur l'économie réelle et les finances publiques encore difficiles à estimer, la situation semble nettement plus délicate. Il est grand temps de rappeler que le développement des clusters ne produit pas que des effets cumulatifs positifs pour l'ensemble de l'économie; en temps de crise, ces structures courent également le risque d'être happées par des spirales descendantes, comme nous l'avons vu lors des crises qui ont frappé les anciennes régions industrielles.

La politique de clusters ne saurait se substituer à une politique macroéconomique. C'est une mésopolitique, qui vise à améliorer les conditions de l'innovation et de l'emploi. Si les conditions cadre sont mauvaises, la politique de clusters ne pourra compenser les défaillances économiques générales. Elle s'inscrit toutefois dans le long terme : de même qu'elle ne peut réussir à court terme, elle ne peut être remise en cause par les crises. Si, comme cela a été formulé dans l'agenda de Lisbonne, la capacité d'innovation et de compétitivité de l'Europe ou des régions européennes dépend de facteurs comme le savoir, l'innovation et la compétence, et que cette capacité peut se développer à l'aide de formes souples de coopération et de réseaux, cela influe également sur la conception de la politique de clusters.

En ce sens, la politique de clusters n'a pas pour objectif la mise en place de projets technologiques ou d'innovations à l'échelle individuelle; sa préoccupation est de transformer plus généralement les institutions socio-économiques afin de préserver les performances dans un contexte de concurrence mondiale (sur le contexte sociologique, voir Boltanski/Chiapello, 2006). Pour éclairer plus concrètement les trois dimensions citées plus haut: concevoir des stratégies et des instruments nouveaux revient à demander comment le politique peut activer les potentiels, s'il est en mesure d'évaluer ses projets de manière réaliste et, le cas échéant, d'y mettre un terme. Le politique n'est plus ici l'instance centrale de pilotage et de décision, mais un acteur parmi de nombreux autres, même s'il demeure 
l'acteur central et moteur d'un point de vue stratégique dans un projet social plus global.

Les structures décentralisées vont de pair avec une gouvernance professionnelle de qualité. On note déjà des avancées importantes, mais il ne s'agit pas uniquement de gérer le développement des clusters. Outre l'orientation stratégique, il est essentiel de gérer les interfaces :

- entre acteurs politico-administratifs décentralisés et centralisés et acteurs des territoires ;

- entre la gouvernance du cluster elle-même et d'autres acteurs de la politique structurelle et d'innovation régionale, autrement dit les acteurs du soutien à la technologie, de l'aide à la création d'entreprises ou à l'emploi ;

- entre acteurs publics et privés, afin d'élaborer et de mettre en œuvre une orientation stratégique commune ;

- entre activités coopératives ou mises en réseau et la concurrence parallèlement toujours nécessaire en matière d'innovation, y compris, précisément, entre des entreprises appartenant à un même cluster ;

- enfin, entre la perspective intérieure régionale et la mise en réseau vers l'extérieur, vitale pour le développement du cluster.

Cette évolution ne peut se produire que si la troisième dimension se réalise dans le même temps: si les entreprises opèrent une réorientation stratégique. Cela suppose pour commencer qu'elles ne changent pas de site à loisir, mais que l'implantation stable soit la règle, de même que l'engagement actif des entreprises dans le développement de leur site ou de leur région. Au niveau européen, cette stabilité est aujourd'hui très variable, mais elle n'est pas si faible que le laisse penser la représentation générale d'entreprises apatrides.

\section{Quelles alternatives ? Du cluster à l'espace d'innovation}

A l'avenir, les clusters auront ou pourront avoir la fonction centrale de pôles dynamiques de l'économie nationale. Mais cela suppose que la politique de clusters ait elle-même des capacités d'innovation et qu'elle parvienne à se concentrer sur ces pôles, cessant de saupoudrer les ressources - comme cela se pratique depuis longtemps en matière de politique structurelle. Il est sans doute important de ne pas laisser la perspective régionale se rétrécir à l'excès. Aucun territoire ne peut aujourd'hui échapper à la division mondiale du travail et, ainsi, les réflexions visant à mettre en réseau la science et l'industrie à l'échelle régionale mènent plutôt à une marchandisation du savoir, détachée des tendances mondiales, qu'à une compétitivité régionale.

On ne peut accroître la capacité d'innovation de l'ensemble de la société en partant prioritairement des régions. Le point de départ devrait plutôt être le processus d'innovation lui-même (schéma 5). Premièrement, une région peut être innovante de bien des manières: non seulement en tant que cluster de technologie ou de 
production, mais aussi, par exemple, par une politique volontariste d'utilisation de nouveaux produits, services ou infrastructures. Ce positionnement présente un intérêt particulier car c'est précisément dans la mise en œuvre de nouveaux produits et services que réside la principale faiblesse de l'Europe en matière d'innovation. Deuxièmement, le processus d'innovation regroupe une multitude de clients, fournisseurs ou entreprises de recherche et développement qui sont loin d'être systématiquement implantés sur les sites des clusters, mais qui sont indispensables pour les innovations. Troisièmement, il existe, indépendamment des différents clusters, un grand nombre de conférences et de congrès qui sont des lieux de communication des nouveaux savoirs, et des réseaux très informels, propres à un métier par exemple, au sein desquels on discute des nouvelles évolutions, réduisant ainsi l'incertitude.

\section{Schéma 5 : Espaces d'innovation}

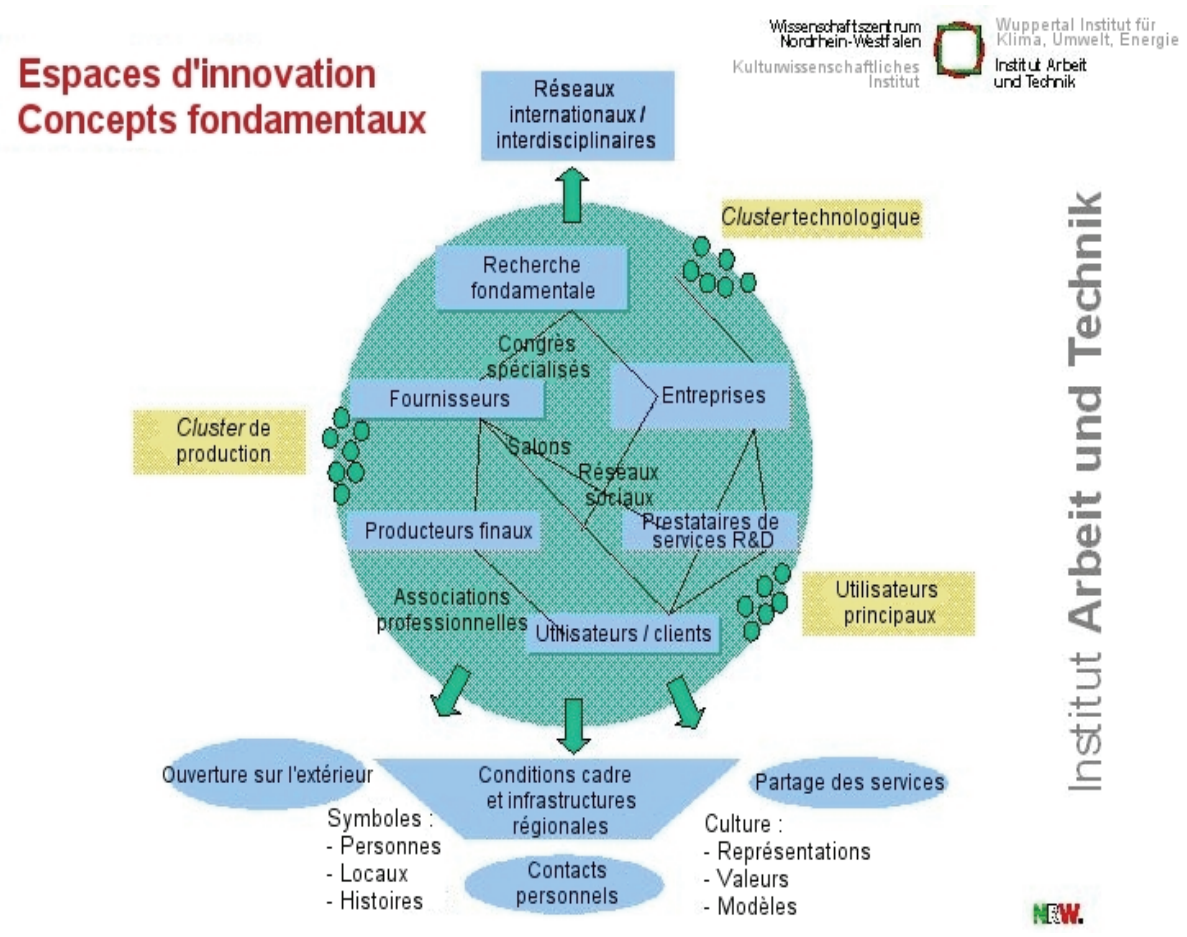

Source : (C) 2007 IAT - présentation de l'auteur.

L'OUVERTURE SUR L'EXTÉRIEUR est donc tout aussi importante pour le bon développement des clusters qu'une base interne créative et souple. En l'absence de ces deux paramètres, les clusters courent le risque de se couper de l'extérieur (effets de " verrouillage ») et de tomber dans une spirale descendante cumulative. Il faudra se demander dans quelle mesure la politique structurelle à 
venir ne doit pas miser sensiblement plus sur ces deux aspects, ouverture sur l'extérieur et créativité à l'intérieur. L'objectif consisterait alors à créer des espaces d'innovation dans le cadre desquels les clusters peuvent jouer un rôle essentiel mais ne sont pas incontournables. Une telle stratégie aurait pour avantage de donner des perspectives stratégiques aux régions, même si elles ne disposent pas des ressources nécessaires au développement d'un cluster.

A l'heure actuelle, aucune stratégie alternative n'étant prévue, les régions qui ont besoin de soutien structurel doivent «s'accorder sur» ou "définir» des clusters. Or, il faut d'urgence des alternatives pour que la politique de clusters puisse prendre les décisions de répartition nécessaires, et se concentrer sur la promotion des pôles qui satisfont à ses objectifs.

\section{Traduction de Marie GRAVEY}

\section{Indications bibliographiques}

Andersson T., Serger S.S., Soervik J., Hansson W.E., The Cluster Policies Whitebook, IKED, Malmö, 2004

Asheim B., CoOKe P., MARTin R. (ed), Clusters and Regional Development, Routledge, Londres/New York, 2006

BENZ A. et al. (ed), Handbuch Governance, VS Verlag, Wiesbaden, 2007

Boltanski L., Chiapello E., Der neue Geist des Kapitalismus, UVK Verlag, Constance, 2006

KRUGMAN P., Geography and Trade, MIT Press/Leuven UP, Londres, 1991

PiORE M., SABEL C., The Second Industrial Divide, Basic Books, New York, 1984

PORTER M. E., The Competitive Advantage of Nations, Free Press Edition, New York, 1990

REHFELD D., Produktionscluster, Hampp, Munich/Mering, 1999

REHFELD., «Global strategies compared: Firms, Markets and Regions», European Planning Studies, vol. 9, $\mathrm{n}^{\circ} 1$, janvier 2001, p. 29-46

REHFELD D., Regionale Kulturen und Unternehmenskulturen, IAT, Gelsenkirchen, 2008

REHFELD D., TERSTRIEP J., Realistische Erwartungen an Clustermanagement. Expertise für die HansBöckler-Stifttung, IAT, Gelsenkirchen, 2007

SCHEUPLEIN C., Der Raum der Produktion. Wirtschaftliche Cluster in der Volkswirtschaftslehre des 19. Jahrhunderts, Duncker\&Humblot, Berlin, 2006

Sölvell O., LindQvist G., KeTEls C., The Cluster Initiative Greenbook, Ivory Tower, Stockholm, 2003

TERSTRIEP J., REHFELD D., Policy Recommendations - Lessons learnt from NICE, Europe innova - innovation and clusters, NICE, IAT, Gelsenkirchen, 2008. 



\section{L’intégration européenne des stratégies nationales}

\section{Alain QUÉVREUX}

Au-delà de la dimension binationale franco-allemande, cette contribution aborde l'enjeu de la coordination des politiques d'innovation des Etats membres au sein de l'Union européenne, dans une logique de concertation et de complémentarité des savoir-faire nationaux.

Peut-on intégrer les stratégies française et allemande alors que tant de différences apparaissent à chaque niveau d'intervention? A défaut d'apporter une réponse immédiate, nous allons au moins essayer de voir comment nous pouvons avancer. La première question à se poser, serait : y a-t-il des stratégies nationales ? Nous avons vu au cours de ce colloque que nous en retrouvions des éléments, mais y a-t-il une stratégie organisée ? Du côté français, il a été annoncé qu'il y aurait une stratégie nationale de recherche et d'innovation au mois d'avril 2009 donc nous pouvons en parler. Il y a donc quand même un début de sujet intéressant l'intégration européenne. Je vais commencer par cet aspect en liant (puisque je viens d'une association qui lie les deux) la recherche et l'innovation et en regardant ce qui se passe actuellement au plan européen et ce que nous essayons de faire. Je mentionnerai les soutiens du gouvernement français.

\section{La recherche : une compétence « partagée » au sein de l'UE}

La première chose est qu'il devient maintenant très clair pour tous, $\mathrm{y}$ compris la Commission européenne, que l'essentiel de ce qui se passe en matière de recherche et d'innovation est fait ou rendu possible par les Etats membres. En matière de recherche, $85 \%$ des actions sont financées par les Etats membres et non pas par la Commission, par des programmes nationaux ou par des programmes transnationaux. Donc, l'essentiel de ce que nous avons devant nous est actuellement d'ordre national. Et là, puisque je m'occupe d'affaires communautaires, je vais peut-être aussi essayer de préciser les mots. La recherche par exemple, du point de vue des traités, est ce qu'on appelle une compétence partagée, mais le problème est qu'en français, on peut comprendre le mot "partagé » de différentes façons. Peut-être la traduction la plus communément admise du mot «partagé » en français, est le mot « à compétences séparées » voire « divisées ». Chacun s'efforce de diviser pour régner chez lui, donc on est dans un univers dont aujourd'hui, nous nous rendons tous compte qu'il est trop fragmenté, trop divisé, trop peu organisé pour être efficace.

\section{Programmation et concertation européennes}

La Commission européenne a fait une proposition au mois de mai 2008 afin que les Etats membres coordonnent leurs politiques. C'est une idée de programmation 
conjointe. Je reviendrai sur ce mot «programmation» qui est un mot compliqué lui aussi. Dans cette idée de programmation conjointe, il y a l'idée de se coordonner, qu'après tout, chacun dans son pays peut prendre les mesures qui lui semblent les plus appropriées à ce qu'il veut faire; simplement il ne doit pas le faire au détriment des autres, de façon clandestine et non coordonnée. Ce que propose la Commission, c'est qu'à géométrie variable, c'est-à-dire lorsque deux, trois, quatre, dix Etats ont l'intention de travailler sur un même problème, on essaye de mettre ensemble la thématique de façon à l'organiser à l'échelle européenne et je dirais que le seul cran pratiquement obligatoire est de regarder ensemble l'agenda de recherche et les possibilités d'innovation. Cette conjugaison des regards n'empêche pas chacun, avec ses propres armes, de tout faire pour tirer le maximum de la stratégie ou de faire de manière isolée quelque chose qui sera peut-être complémentaire à ce que feront les autres. Il y a toute une série de graduations possibles lorsque l'on décide de s'intégrer, de travailler ensemble, mais ce que propose la Commission est finalement quelque chose d'assez modeste qui consiste simplement à dire : nous allons nous concerter et après, chacun fera ce qu'il veut, mais pas contre les autres. J'ai entendu Madame Merkel dans un autre sujet se fâcher contre un petit pays de l'Union européenne qui est plutôt du côté ouest de l'Union européenne et qui avait pris des décisions qu'elle ne critiquait pas sur le fond, mais qu'elle critiquait sur la forme, c'est-à-dire sur l'absence de concertation préalable, en prenant le risque de fragiliser l'ensemble des pays européens. C'est cela qui nous est proposé en termes d'intégration européenne. Je crois qu'à aucun moment, ni par la Commission ni nulle part dans les Etats membres, on n'avait envie d'être tous pareils et de réagir tous aux mêmes injonctions, ni d'avoir tous les mêmes façons de travailler. Ce qui nous est proposé aujourd'hui, c'est de définir ce que chacun veut faire, avec qui il veut le faire, et après nous pourrons voir comment nous voulons le faire parce qu'il y a aussi des différences de trajectoires, de systèmes et il y a parfois des différences dans les modalités d'application, l'organisation des programmes et la délivrance des financements pour des raisons qui sont issues de l'histoire de nos pays. Toutefois je crois qu'il serait possible de mettre en œuvre, avec des outils relativement similaires, des grands programmes allemands, français, ou encore finlandais. Probablement, il doit être possible rien qu'en échangeant, de mieux travailler ensemble.

Il reste ensuite une question difficile, qui est: qu'est-ce qu'on va faire ensemble et jusqu'où va-t-on le faire ensemble ? Ce qui semble certain, c'est qu'il faut faire les orientations ensemble, c'est-à-dire les grands choix politiques. Je pense qu'on va vite aboutir à la conclusion que l'Europe n'a pas tant d'argent que cela, qu'on ne peut pas tout faire et en particulier qu'aucun Etat membre ne peut tout faire, même le plus grand. Par conséquent, il faut faire des choix, c'est-à-dire renoncer. Faire des choix, cela ne consiste pas à dire ce qui est important, car c'est facile de dire ce qui est important. Ce qui est difficile, c'est de dire ce que l'on ne fera pas et si on ne le fait pas, qui le fera et comment on travaillera avec d'autres partenaires. 


\section{Complémentarité des stratégies nationales...}

Finalement, ce qu'on cherche à faire, si on veut penser intégration européenne et stratégies nationales et si on pense que durablement il y aura des stratégies nationales et qu'elles ont intérêt à coordonner leurs efforts, c'est certainement d'améliorer la connaissance et la compréhension de ce que font les autres car souvent ils poursuivent les mêmes objectifs que nous. Si on veut un instant supposer le problème résolu: nous sommes en 3000, les deux stratégies nationales, allemande et française, sont parfaitement coordonnées, qu'avons-nous réussi à faire? Nous avons réussi à faire que chacun, là où il est, est devenu le grand spécialiste mondial de ce qu'il sait faire le mieux avec les meilleurs partenaires possibles puisque, et c'est aussi le thème de la stratégie " Hautes Technologies » en Allemagne, il faut travailler avec ceux qui vous sont complémentaires.

Ce qu'on va chercher dans un partenariat franco-allemand ou dans une intégration européenne des stratégies nationales ne va pas être de déterminer une stratégie unique pour l'Europe, mais de faire en sorte que les stratégies différentes se connectent pour produire mieux, pour produire plus, pour garder à l'Europe la prospérité qu'elle a acquise dans le passé et qui ne lui est pas garantie pour l'avenir par rapport aux autres régions du monde. Ce qu'on va essayer de faire, c'est de travailler sur ces connections ; on va essayer d'améliorer les connections. Je fais des efforts pour ne pas utiliser le mot «réseau» qui me viendrait facilement à la bouche parce qu'il ne s'agit pas de faire en sorte que tout le monde soit partout avec tout le monde; il s'agit d'être également assez sélectif. Si on pense à une intégration stratégique, l'idée est bien de faire travailler ensemble des gens qui sont complémentaires. Plus que le dialogue entre les pairs, ce qui est important, c'est de se connecter avec des gens différents de soi et dans un but précis qui est de fabriquer les spécialisations de demain, les grandes spécialités mondiales. Il y en a certaines aujourd'hui qui existent et qui sont largement héritées de l'histoire et de l'économie industrielle. Il y en a des nouvelles qui sont en train de naître dans les pays émergents et même dans nos pays parce qu'on est en train de passer à l'économie de la connaissance, à l'économie des complémentarités entre les idées et entre les systèmes. Ce qu'on va essayer de faire, c'est de définir ensemble ou de regarder ensemble quelles sont les grandes stratégies des acteurs pour les intégrer peut-être au niveau européen.

Il y a souvent dans les textes de la Commission européenne - il y a toujours j'allais dire - sur cette question-là de grands oubliés. Les grands oubliés, ce sont les peuples européens. Personne ne s'intéresse, dans les textes en tout cas, à ce que pensent les Européens, à ce qu'ils pourraient attendre d'une telle intégration des spécialités et des compétences. Je pense que cela est aussi un point qui devra être traité si on espère que notre intégration européenne, que notre conjugaison des compétences nationales, puisse se faire à grande échelle et avec une grande ambition internationale. Certainement, il va falloir trouver les moyens d'associer les citoyens européens à la recherche, aux innovations. 


\section{...et coordination à géométrie variable}

Nous découvrons aujourd'hui de façon dramatique dans le monde de la finance que l'innovation financière est systémique elle aussi comme toute innovation, que nos Etats sont systémiques. Cela peut faire très mal. La caractéristique d'un système est qu'il faut tout changer à la fois et qu'à l'inverse, quand cela commence à mal fonctionner, tout peut échouer par effet domino. Si nous voulons intégrer nos stratégies, nos systèmes, les faire cohabiter, il va falloir définir les outils qui permettront à la base, sur le terrain, région par région, langue par langue, thème de recherche par thème de recherche, de voir quels sont les grands agendas et comment on passe de ces grandes idées, de ces grands travaux, à une économie performante, une économie tirée par l'innovation. Ce sont beaucoup de défis. Si je rajoute les uns après les autres les mots que je prononce, mais qui ne sont en fait pas prononcés par moi, que je reprends d'autres, on voudrait construire la géométrie variable des systèmes hétérogènes (je comprends que vous appeliez cela théorique parce que c'est difficile). Je crois qu'il va falloir revenir à des outils concrets comme il en existe au plan européen, les ERA-Net (European Research Area Networks) qui sont les coordinations des politiques nationales à géométrie variable. Il existe déjà des programmes, ou il va exister par exemple dans le domaine de l'assistance à domicile, dans les technologies de l'information un programme lui aussi qui associe les Etats.

Maintenant, ce que propose la Commission européenne, c'est que les Etats travaillent entre eux, sans elle ou avec elle en tant que catalyseur, et sans financement communautaire. Je ne voudrais pas vous faire rire, mais la Commission a dit qu'il ne fallait pas d'argent communautaire car s'il y avait de l'argent communautaire, cela introduisait tellement de bureaucratie que c'était contreproductif. Que la Commission dise cela pouvait prêter à sourire. Nous, les Européens sommes-nous capables, secteur par secteur, organisme par organisme, de construire le monde, l'internationalisation dont nous avons besoin pour rester compétitifs à l'échelle mondiale ? Nous avons dans nos deux pays de très grandes organisations de recherche et de technologie qui sont probablement les forces qui vont structurer la nouvelle économie ou bien alors nous allons disparaître. C'est une autre option!

Certainement, en conjuguant les façons de travailler, on va s'apercevoir que l'on peut définir des programmes ensemble. Probablement, le prochain chantier pour le CIRAC est : comment va-t-on faire pour avoir des orientations communes puisque nous ne construisons pas de la même façon? Et là, je séparerais deux niveaux. Le premier niveau qui est vraiment l'orientation politique elle-même : de dire par exemple les Européens considèrent que l'Alzheimer est une question importante, les maladies neuro-dégénératives sont une question importante, mais pas de rester au niveau de dire: il nous faut une planète propre et un vieillissement maîtrisé. J'y suis favorable, mais ce n'est pas suffisant. A quel prix ? Que peut-on faire et sur qui s'appuyer pour les aspects que nous ne développerons pas nous-mêmes? Et puis il $\mathrm{y}$ a un deuxième niveau que 
j'appellerais celui de la programmation elle-même. D'ailleurs, il y aurait un troisième niveau qui serait la mise en œuvre. Une fois qu'on a dit dans quel domaine étaient situées les priorités européennes ou les priorités d'un groupe d'Etats, la performance finale ne viendra que si les acteurs de ces Etats, les grands acteurs de la recherche et de l'innovation dans ces Etats, se mobilisent utilement. Pour qu'ils se mobilisent utilement, il est nécessaire de les avoir associés intimement à la préparation de la mise en œuvre des programmes et même à la conception des programmes dans le respect des orientations politiques. Je crois qu'en franco-allemand, en bilatéral ou en multilatéral à géométrie variable, c'est sur ce cran qu'il faut le plus travailler : mettre ensemble les dirigeants des Etats membres, même dans une configuration limitée avec quelques uns des Etats membres, nous savons le faire, ils savent le faire et ils savent parfois se mettre d'accord sur les grandes orientations. En revanche, il est difficile d'arriver à définir une façon de travailler conjointe, voire commune. Pourrait-on admettre que dans un programme allemand les participants français soient financés? Pourrait-on admettre que dans un programme français les participants allemands soient financés? Peut-être que oui. Le comble de l'horreur pour nos administrations nationales, c'est de faire un pot commun, de mettre l'argent dans un même endroit. D'une façon générale, je crois que c'est un peu ce type de questions qui se pose : fait-on un pot commun ou pas? A-t-on une stratégie partiellement conjointe ou pas ? Peut-on la caractériser?

Français et Allemands ont leur histoire. L'Allemagne a mis en place une stratégie des hautes technologies, la France réfléchit à une stratégie nationale de recherche et d'innovation. Peut-être que parfois, on peut parler plus franchement en Allemagne qu'en France, mais dans les deux cas, on cherche l'excellence et on cherche à renforcer les acteurs qui ont la capacité de nous tirer à l'échelle internationale, dans les deux cas en associant les meilleures universités, les meilleurs organismes de recherche, les entreprises les plus puissantes voire les PME les plus dynamiques. Donc, on a bien tout un ensemble de choses qui sont les mêmes en France et en Allemagne, qu'on essaye de faire travailler ensemble et en coopération internationale. Il devient possible à partir de ces éléments qui sont certes disparates ou différents dans les deux pays, vu qu'ils sont mobilisés dans les deux pays avec des outils différents - c'est là que réside la faiblesse -, d'envisager des connexions transnationales et que la stratégie dans les nanotechnologies par exemple soit faite ou tirée ou organisée principalement par un grand organisme allemand, français ou belge. Il y a des possibilités de cette façon d'organiser la spécialité européenne dans les nanotechnologies - ce serait pareil dans d'autres domaines - à partir d'un certain nombre de lieux et d'organisations qui se sont alliés.

Je ne sais pas si vous connaissez le plan dans le domaine de l'énergie de la Commission européenne ou ce qu'elle essaye de faire (le SET Plan). Dans le domaine de l'énergie, il y a plusieurs voies technologiques possibles, on ne sait pas exactement laquelle choisir, mais quel(s) que soit le ou les choix, il(s) sera/seront de toute façon très coûteux en termes d'investissement. Il est donc important de ne pas 
aller dans n'importe quelle direction. Ce qui est en train d'apparaître, c'est qu'y compris du point de vue des très grandes entreprises multinationales, y compris du point de vue des décideurs politiques parce qu'ils sont conscients des enjeux et tout à fait incapables de pouvoir choisir entre les voies technologiques, il devient tout à fait essentiel qu'il y ait une sorte d'organisation, d'alliance des grands acteurs de la recherche européenne dans le domaine de l'énergie pour éclairer le chemin, pour dire : nous pouvons prendre telle voie ou telle autre, il y aura telle conséquence ou il y aura telle conséquence. Dans ce domaine, le danger est que nous ne savons pas ce qu'il se passerait si... Dans cet autre domaine, nous ne savons pas ce qu'il se passerait si... Donc, il y a une limite, notre rationalité est limitée. Nous ne disposons pas de toutes les informations qui nous permettent de décider en toute tranquillité. Pourtant, il va falloir décider et prendre une voie coûteuse parce qu'elles sont toutes coûteuses.

Plus globalement, j'ai un peu l'impression que derrière les outils mis en place avec beaucoup de volonté mais de manière encore tâtonnante dans chacun de nos Etats, nous cherchons tous à réformer en profondeur nos systèmes en mobilisant les mêmes forces pour aboutir à des partenariats adaptés au monde en train de se reconstruire. On s'aperçoit que très lentement, le rôle d'une recherche publique très internationalisée comme moteur de l'économie, de la construction du nouveau système économique est en train de naître. Cela devient l'intérêt de tout le monde de coordonner plus intensément les efforts. Quand je regarde la façon dont les organismes de recherche travaillent entre eux, ils sont différents, ils ont chacun leurs qualités, chacun leurs défauts. Autour de grands enjeux, il devient possible de fédérer la construction des solutions européennes qui pourront être mises au service d'une politique forcément très intelligente. En prenant cette voie d'intégration des stratégies européennes tout en respectant les différences et les façons d'être de chacun des partenaires, nous avons la capacité de changer la donne et de reconstruire.

MON PROPOS ICI SERAIT DE DIRE : c'est en train de bouger dans les Etats, c'est en train de bouger au niveau de l'Union. On commence à entrevoir une possibilité de construire l'économie de la connaissance sur une base globale et mondiale différemment de ce qui a déterminé l'économie industrielle dans laquelle nous vivons aujourd'hui. Chacun comprend qu'elle a atteint ses limites et qu'elle est en train plus ou moins de s'effondrer. Je m'arrêterai là. Pour confirmer ce que vous avez dit en introduction de cette table ronde et en même temps polémiquer avec vous, oui je constate des différences entre nos systèmes qui semblent abyssales, mais non, ce n'est pas une catastrophe. On voit aussi des germes d'un dépassement de ces différences dans des stratégies plus intégrées et très efficaces, car nos objectifs sont les mêmes. 


\section{LES AUTEURS}

\section{Isabelle BOURGEOIS}

Chargée de recherches au CIRAC, Cergy-Pontoise

\section{Horst DOMDEY}

Président de Bio ${ }^{\mathrm{M}}$ Biotech Cluster Development GmbH

\section{Bernard FROMENT}

Chef du département partenariats et valorisation, Service des Entreprises, du Transfert de Technologie et de l'Action Régionale, Direction Générale pour la Recherche et l'Innovation, Ministère de l'Enseignement Supérieur et de la Recherche (jusqu'en 2009)

Actuellement Inspecteur général de l'administration de l'Education nationale et de la Recherche

\section{Michael GROB}

Président de BC Brandenburg Capital GmbH (jusqu'en 2010)

\section{Sebastian HANNY}

Coordinateur du réseau de création d'entreprises des universités de Dortmund, Unité de transfert de connaissances de l'Université technique de Dortmund

\section{Solène HAZOUARD}

Ingénieur d'études au CIRAC, Cergy-Pontoise

\section{Sonja R. KIND}

VDI/VDE Innovation + Technik GmbH, Section Socio-économique

\section{Marianne KULICKE}

Chef de projets, Centre de compétences « Politique et Régions », Institut Fraunhofer de Recherche sur les Systèmes et l'Innovation (ISI), Karlsruhe

\section{René LASSERRE}

Professeur à l'Université de Cergy-Pontoise, Directeur du CIRAC, Cergy-Pontoise

\section{Emmanuel LEPRINCE}

Délégué général du Comité Richelieu (jusqu'en 2010)

Actuellement Délégué Général de l'association Pacte PME

\section{Jean-Yves MÉRINDOL}

Directeur de l'ENS Cachan

\section{Petra Moog}

Titulaire de la chaire d'économie générale d'entreprise, Spécialité succession d'entreprises, Université de Siegen 


\section{Rainer NÄGELE}

Membre du bureau de l'Union de la recherche (Forschungsunion), Directeur du Centre de compétences Management de services, Institut Fraunhofer sur l'Economie du travail et l'organisation (IAO), Stuttgart

\section{Pierre PAPON}

Professeur émérite à l'Ecole Supérieure de Physique et de Chimie Industrielle (ESPCI), Président du groupe de travail FutuRIS 2006-2007 sur l'internationalisation des activités de $R \& D$ et d'innovation et son impact sur les territoires, Association Nationale de la Recherche et de la Technologie (ANRT)

\section{Alain QUÉVREUX}

Responsable du service Europe, Association Nationale de la Recherche et de la Technologie (ANRT)

\section{Dieter REHFELD}

Responsable des thèmes de recherche Innovation, Territoires et Culture, Institut du Travail et de la Technique (IAT), Gelsenkirchen

\section{Ulrich SCHMOCH}

Chef de projets, Centre de compétences « Politique et Régions », Institut Fraunhofer de recherche sur les systèmes et l'innovation (ISI), Karslruhe

\section{Mark SELLENTHIN}

Département d'économie industrielle et de management international, Centre d'études économiques européennes (ZEW), Mannheim (jusqu'en 2010) Actuellement Professeur d'économie à la Fachhochschule de Coblence

\section{Hubert STEIGERWALD}

Directeur de Sensorik Bayern GmbH

\section{Christoph THIEME}

Chercheur à l'Institut de Conseil et de Recherche Sociale Appliquée (IPRAS), Université d'Iéna

\section{Henrik UTERWEDDE}

Directeur adjoint de l'Institut Franco-Allemand de Ludwigsburg (DFI)

\section{Thierry WEIL}

Responsable de l'équipe "Management de l'innovation", Centre d'économie industrielle de l'Ecole des Mines de Paris, Chercheur à l'Observatoire des pôles de compétitivité

\section{Peter WEINGART}

Professeur de sociologie,

Chercheur à l'Institut pour la recherche scientifique et technique (IWT),

Université de Bielefeld 


\section{Composition : CIRAC}

www.cirac.u-cergy.fr

CIRAC, c/o Université de Cergy-Pontoise

33 boulevard du Port - 95011 CERGY-PONTOISE CEDEX

Impression : Dupli-print

2 rue Descartes - Z.I. Sezac - 95330 DOMONT

Dépôt légal : décembre 2010

Imprimé en France 



\section{LES PO LITIQUES D ' INNONATIO N C OOF ERATTVE EN ALIEMAGNE ET EN FRANCE}

\section{Expériences et approches comparées}

\section{Sous la drection de Solène HAZOUARD, René LASSERRE, Herrik UTERWEDDE}

L'irmoration se sitie aucoeux des stratégies économiquer corternqoanines. Dans un contexte de changemerts profonds et acólérés par la mondilisation et Pémergenoe de nouxealx concunerts, par les rath tions technologiques et les perspectiver ouxerter par l'economie de h conrassanoe, enfin par les problèmes écologiques et les défis du déseloppement darable,

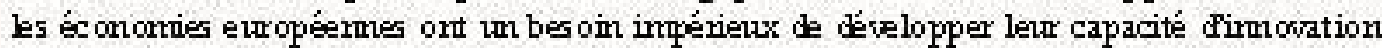
poux faire froe à oes défis multiples. Il yva de lexx conquétitivité, du ruintien de leux potentiel ferquloi et de croisance et, au-deli, de bur capacité de présererer, tout en les adaptart, leux modèler éconorriques et $50 \mathrm{cin} u x$

L'Allerngne s'attache depuri bientốt dewx décenries à promolwoir une moderrisation systérique de son dispositif de recherche et firmotation, non seulement en y corsacrart dor moyers aocns, articulés a utour dfaxes statégiques significatif, mis également en mettant en

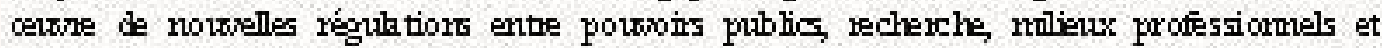
entrepriser Le pays privilégie ainsi une politique firmorationcoopération, fondée sux der réseanx de coopéntion enthe les acteuss (résealx de corquétences, dasters, coopéna tiors intenentrepriser intúgant les PME). Darr une dérnarche parallèle, la Fance se concertre principalemert, depuris 2004 , sur la rưe en phce et ha promotion der pôler de corquétitivité

Faoe au défi actiel de h núe en commun des potentiel firmoxation rationalx à Péchelle

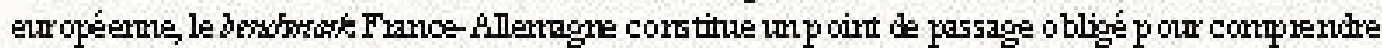
et convilier les donaniquiver firmonation respectiver, nenforcer les coopéntions et inquaker br synerges franc o-a lemander qui contribueront à h conquetitivité globale de PUE. Auregard de cet erjeu décisif le prérent ournage se propose de rendre compte des orientations et der démancher

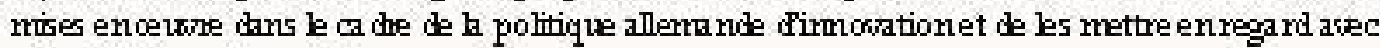
oelles développéer en France

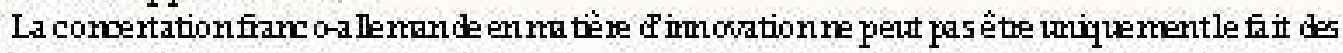

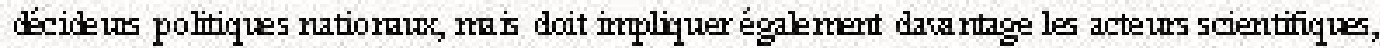
économiques et tenit oriaxx, à Pinstar de ce qui se pratique outre-Rlim et constitue le poirt fort dh système allerrand de recherche et firmoration. A tavers oe linge, nour avors voulu montrer concrètemert, en nour appryant sur la parole des acteurs, Firtérêt dhx cas allenand et traoer lor woir d'ume meilleure corwergenoe entre deux pays parternirer de poict qui devaient s'attacher,

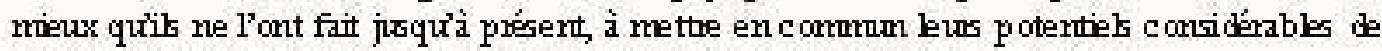
recherche et firmoration, au profit de h corrqétitivité et de laverir de l'Europe

Ororage publié atec le concours du CIERA et du CICC (Urixerité de Cengy-P ontoise)

$$
25 € \mathrm{TTC}
$$

ISBN : 978-2-905518-39-2
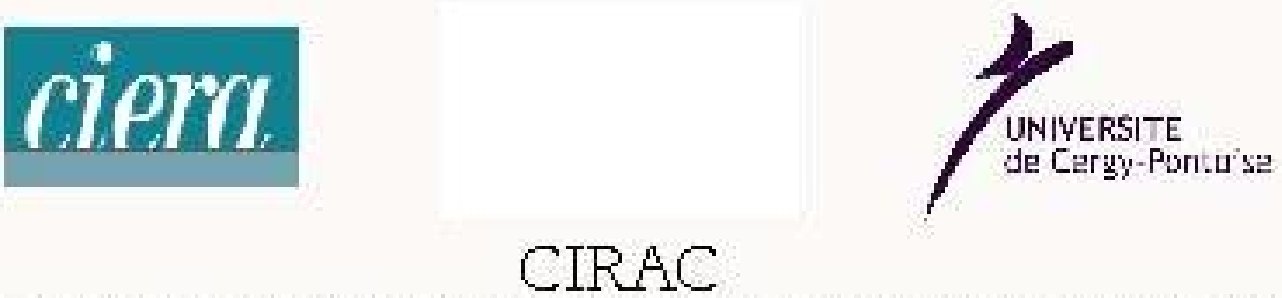